\title{
AMPK-glycogen interplay: an opportunity for drug design
}

Citation for published version (APA):

Miglianico, M. (2015). AMPK-glycogen interplay: an opportunity for drug design. [Doctoral Thesis, Maastricht University]. Maastricht University. https://doi.org/10.26481/dis.20151023mm

Document status and date:

Published: 01/01/2015

DOI:

10.26481/dis.20151023mm

Document Version:

Publisher's PDF, also known as Version of record

\section{Please check the document version of this publication:}

- A submitted manuscript is the version of the article upon submission and before peer-review. There can be important differences between the submitted version and the official published version of record.

People interested in the research are advised to contact the author for the final version of the publication, or visit the DOI to the publisher's website.

- The final author version and the galley proof are versions of the publication after peer review.

- The final published version features the final layout of the paper including the volume, issue and page numbers.

Link to publication

\footnotetext{
General rights rights.

- You may freely distribute the URL identifying the publication in the public portal. please follow below link for the End User Agreement:

www.umlib.nl/taverne-license

Take down policy

If you believe that this document breaches copyright please contact us at:

repository@maastrichtuniversity.nl

providing details and we will investigate your claim.
}

Copyright and moral rights for the publications made accessible in the public portal are retained by the authors and/or other copyright owners and it is a condition of accessing publications that users recognise and abide by the legal requirements associated with these

- Users may download and print one copy of any publication from the public portal for the purpose of private study or research.

- You may not further distribute the material or use it for any profit-making activity or commercial gain

If the publication is distributed under the terms of Article $25 \mathrm{fa}$ of the Dutch Copyright Act, indicated by the "Taverne" license above, 
AMPK-glycogen interplay:
an opportunity for drug design 
(C) Marie Miglianico, 2015. For all articles published, the copyright has been transferred to the respective publisher. No part of this publication may be reproduced, stored in a retrieval system or transmitted in any form by any means, without prior written permission from the author, or when appropriate, from the publisher.

ISBN: 978-90-9029282-3

Cover design and layout by: Tomasz Karawajczyk and Marie Miglianico 


\title{
AMPK-glycogen interplay: an opportunity for drug design
}

\author{
PROEFSCHRIFT
}

ter verkrijging van de graad van doctor aan de Universiteit Maastricht, op gezag van de Rector Magnificus, Prof. Dr. L.L.G. Soete volgens het besluit van het College van Decanen,

in het openbaar te verdedigen

op vrijdag 23 Oktober 2015 om 10.00 uur

door

Marie Miglianico

Geboren op 3 juli 1987 te Rennes 


\section{Promotor}

Prof. dr. J.F.C. Glatz

\section{Co-promotores}

Dr. D. Neumann

Dr. G.A.F Nicolaes

\section{Beoordelingscommissie}

Prof. dr. T.M. Hackeng (voorzitter)

Dr. W.M. Blankesteijn

Prof. dr. H.P. Brunner-La Rocca

Prof. dr. U. Schlattner (INSERM, Grenoble, Frankrijk)

Prof. dr. H.J.M. Smeets

Financial support by the Dutch Heart Foundation for the publication of this thesis is gratefully acknowledged.

Publication of this thesis is further supported by Stichting Hartsvrienden RESCAR. 


\section{Contents}

$\begin{array}{lll}\text { Chapter } 1 \text { General Introduction } & 7\end{array}$

Chapter 2 Novel targeting opportunities for AMP-activated protein kinase: 25 isoform specificity and computer-aided drug design

Chapter 3 The recruitment of AMP-activated protein kinase to glycogen is regulated by autophosphorylation

Chapter 4 The interaction between AMPK $\beta 2$ and the PP1-targeting subunit $\mathrm{R} 6$ is dynamically regulated by intracellular glycogen content

Chapter 5 Displacing AMPK from glycogen by pharmacological targeting of its carbohydrate-binding module

Chapter 6 General discussion

List of abbreviations

Summary

Samenvatting

Résumé

Valorization

Curriculum vitae

Acknowledgments 

Chapter 1

General Introduction 


\section{Introduction}

One of the major traits of life is the ability to synthesize and metabolize biomolecules. In heterotrophic organisms such as mammals, degradation of carbon sources is the only way to obtain energy while synthesis of complex molecules is a way to both store energy reserves for later use and to allow complex biological processes [1]. Catabolic and anabolic reactions therefore occupy the two sides of a carefully orchestrated and monitored balance of energy production and allocation [2]. At the cellular and whole body level, this metabolic homeostasis controls through a wide set of signaling molecules and pathways feeding and fasting, storing and burning, growing and resting [2]. If the balance between these events is tipped over, serious diseases can occur: notably in type 2 diabetes the energy intake, via that of nutrients, exceeds the energy expenditure, which sets off the whole equilibrium throughout the body and paves the way to cardiovascular diseases [3]. Re-equilibrating the balance requires a change in lifestyle, and in practice is often coupled with pharmacological intervention. Although several pharmacological medications exist to help re-establishing health in type 2 diabetic patients, the complex regulation of metabolic diseases still leaves room for improvement and innovation.

In the following section, we will elaborate on the mechanisms at play in such metabolic disorder and then focus on one of the most important players of the energy homeostasis, the AMP-activated protein kinase (AMPK), finally we will present the main steps of a drug design process to target AMPK.

\section{Metabolic diseases: an energetic imbalance leading to cardiovascular diseases}

On top of genetic susceptibility traits, metabolic disorders may originate from a too high energy intake compared to energy expenditure, i.e., overnutrition coupled with a sedentary lifestyle [4]. At first, excess nutrients are stored as triacylglycerol in the adipose tissue, through hyperplasia and hypertrophy of this tissue. When the maximal capacity is reached, nutrients will overflow into other tissues which are not meant for such storage, such as liver and muscle, leading to so-called ectopic fat accumulation [1]. Subsequently, insulin resistance develops in these tissues, meaning that nutrients are no longer efficiently cleared from the blood into the cells after a meal. The resulting hyperglycemia is further supported by the continuous hepatic glucose production, normally turned off by insulin upon feeding, and the hyperlipidemia is reinforced by release of fatty acids from the adipose tissue stocks [3]. Similarly to insulin, other signaling molecules exert a dampened response in this condition compared to the healthy state. Notably, among the cytokines released by adipose tissue, adiponectin is 
unable to induce fatty acid oxidation in muscle and leptin fails to regulate appetite in the brain [3].

The exact cause for the development of insulin resistance is difficult to establish due to the existence of 3 interconnected factors. Firstly, the accumulation of intracellular lipid is not the cause per se of metabolic inflexibility: athletes for instance have high levels of intramyocellular triacylglycerol and yet preserved insulin sensitivity [5]. By knock-out of different genes involved in the synthesis of the lipid stores, intermediary lipid products, such as diacylglycerol and ceramide, were identified as a more likely link between ectopic fat storage and insulin resistance. These intermediary lipids act as second messengers in cells and trigger pathways that inhibit that of insulin. Secondly, the hypertrophy of adipose tissue and the hepatic steatosis cause the release of proinflammatory cytokines by adipocytes and hepatocytes, which triggers the infiltration of immune cells into the respective tissues [1]. Prolonged persistence of this condition will result in a chronic inflammation state where immune and local tissue cells activate each other. Thirdly, activation of the unfolded protein response (UPR) sets on stress pathways which further dampen the insulin response [6]. In pancreatic cells, the reduced insulin responsiveness drives an increase in insulin production, which leads, through the accumulation of unfolded protein, to the UPR. In other tissues, this state of endoplasmic reticulum stress is highly associated to metabolic disorders but the cause is unexplained and it seems to only be a maladaptive response.

All three components of the insulin resistance result in a vicious cycle, which further worsens the energy homeostasis and metabolic flexibility. To compensate the lack of tissue target responsiveness, pancreatic $\beta$ cell islets expand and produce more insulin which triggers hyperinsulinemia, but the $\beta$ cells eventually die, resulting in a full blown diabetes type 2 phenotype where no insulin is produced anymore [4]. Without even reaching the diabetic state, the constant hyperglycemia, hyperlipidemia and chronic inflammation induce very high risks of developing cardiovascular diseases such as atherosclerosis and heart failure [3]. In summary, even a small imbalance in energy metabolism can lead in the long term to dramatic effects on human health.

\section{AMPK, an ancient system for metabolic balance}

\section{A beginning in evolution as a glucose sensor}

AMP-activated protein kinase (AMPK) is an essential player of energy metabolism and as such, the trimeric protein complex exists in essentially all eukaryotes: as SNF1 (sucrose non fermenting 1) in yeast, SnRK1 (Snf1-related protein kinase 1) in plants, and AMPK in animals [7,8]. In yeast, Snf1 is required to achieve the change in metabolism upon glucose deprivation. Under normal glucose conditions, yeast relies on glycolysis to produce ATP and if glucose runs low, growth can be sustained by 
switching to oxidative metabolism and use of other carbon sources. Without the Snf1 complex however, derepression of gene expression needed for this switch is not possible. In plants, the pathway to produce energy changes with the day/night cycle: with light, plants rely on photosynthesis and in the dark, on respiration. Similarly to regulation in yeast, the process to switch energy source depends on the AMPK-ortholog SnRK1 complex [8]. Therefore, the AMPK related complexes are responsive to starvation and to nutrient availability and trigger the adaptation to these stimuli. In mammals, AMPK is similarly sensitive to nutrient presence and is inhibited by high levels of glucose, lipid or amino acid [9]. However, the main factor activating the kinase is energy stress, notably with reduced levels of ATP and increased levels of that of AMP. In all cases, AMPK is a major player for the switch of energy sources, called metabolic flexibility, and for adaptability to the environment.

\section{AMPK implication in metabolic homeostasis}

First identified as a kinase involved in lipid metabolism, AMPK is now known to be a central hub of the cellular energy homeostasis with roles in carbohydrate and protein metabolism as well [10]. To draw a simple picture of the kinase, under energy-requiring conditions, AMPK switches off unnecessary anabolic pathways while replenishing ATP stocks by boosting catabolic ones. In the case of nutrient metabolism, AMPK activation firstly promotes the translocation of vesicles containing the glucose and fatty acid transporters (GLUT4 and CD36, respectively) to the membrane, which causes an increased nutrient uptake by muscle and liver [10]. Secondly, glycolysis and fatty acid oxidation, which are upregulated by AMPK, catabolize the nutrients, thereby producing energy [11]. Simultaneously, AMPK turns off hepatic glucose output in the liver by prevention of the expression of gluconeogenic genes, and limits lipolysis in adipose tissue by direct targeting of the hormone sensitive lipase [8]. Regarding protein metabolism, AMPK inhibits the mammalian target of rapamycin (mTOR) pathway and downregulates protein synthesis [12]. Lastly, AMPK controls the life and death of mitochondria, by regulating biogenesis and mitophagy, thereby ensuring the ATPproducing capacity of the cell [12].

Taken together, AMPK has roles in nutrient uptake similar to that of insulin, but rather than triggering storage, the nutrients are principally used for energy production in the former pathway (Fig. 1) [2]. Even though some of the downstream targets may overlap, the AMPK pathway is independent of that of insulin and can even act in insulin resistant tissues. Although AMPK activity was reported to be downregulated in insulin resistant tissues and diabetic patients [9], the AMPK activator AICAR is able to reactivate the kinase. Administration of AICAR improves metabolic parameters in a number of genetic rodent models of metabolic disorders, as well as increases insulin sensitivity in 

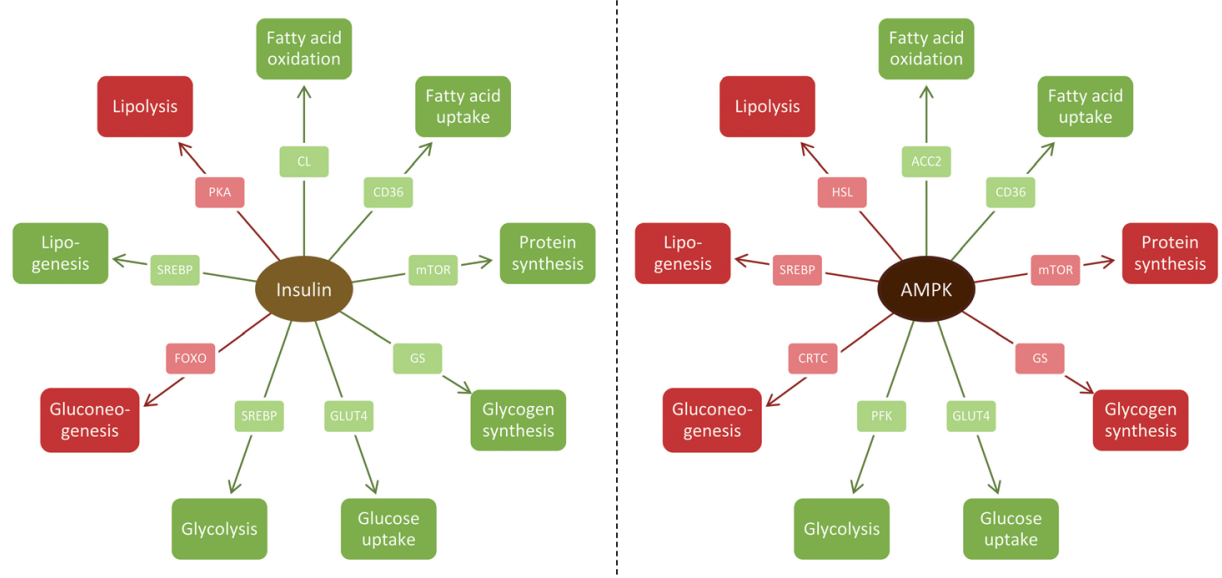

Figure 1. Comparison between AMPK and insulin effects on metabolic pathways.

The pathways activated by each signaling hub are colored in green whereas those inhibited are in red. The intermediate rectangle indicates one of the involved downstream targets (whether it depends on inhibition or activation of this target is not mentioned here). The figure is adapted from $[1,15,16]$ for the insulin part and from $[8,10,11]$ for the AMPK part. CL: citrate lyase, ACC2: acetyl-coenzyme A carboxylase 2, CD36: fatty acid transporter, mTOR: mammalian target of rapamycin, GS: glycogen synthase, GLUT4: glucose transporter 4, SREBP: Sterol regulatory element-binding protein, PFK: phosphofructokinase, CRTC: CREBregulated transcription coactivator FOXO: Forkhead box protein O, PKA: protein kinase A, HSL: hormone sensitive lipase.

insulin-resistant high fat fed rats [13]. In line with the positive effect of AMPK on metabolic parameters, mice with a deletion of one of the two AMPK isoforms present with high plasma glucose levels and are insulin resistant, showing the importance of AMPK in basal energy balance [14].

Beyond nutrient storage problems, insulin resistance also creates chronic inflammation. Interestingly, AMPK activation antagonizes the conversion of macrophages into the pro-inflammatory profile M1 upon fatty acid exposure [8]. Moreover, the increased fatty acid oxidation induced by the direct AMPK activator A-769662 contributes to a reduced release of pro-inflammatory cytokines by macrophages [8].

A change in lifestyle towards a lower calorie intake and more exercise is one of the keys to counteract a metabolic imbalance [4]. Importantly, AMPK activation is involved in exercise-induced metabolic changes. The AMPK activator AICAR mimics the effects of acute exercise on skeletal muscle and chronic AICAR treatment improves endurance. Besides, the increased glucose uptake and fatty acid oxidation linked to exercise are 


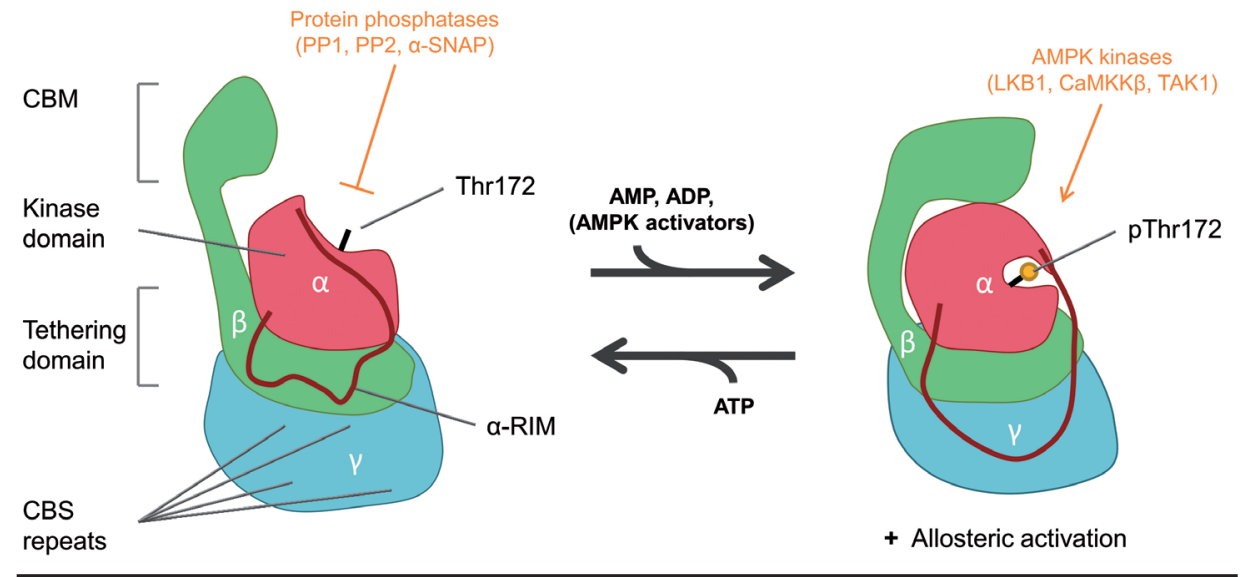

Figure 2. Overview of AMPK structure and regulation by adenine nucleotide.

Schematic representation of AMPK structure (inspired from recent structural data $[18,19])$. The $\alpha, \beta$, and $\gamma$-subunits are represented in red, green and blue respectively. The left panel represents AMPK in an inactive state, with the Thr172 site exposed to phosphatase activity and a disordered $\alpha$-RIM. The right panel represents AMPK in active state following AMP/ADP binding: this state translate into a conformational change, which promotes Thr172 phosphorylation (pThr172) by kinases and protection of pThr172 from phosphatase, as well as, for AMP, allosteric activation. Indirect activators such as metformin raise the level of AMP and thereby trigger the same mechanism of action.

dependent on AMPK activation [13]. Similarly, AMPK is involved in the beneficial effects of caloric restriction [17]. Low nutrient availability activates AMPK and thereby triggers metabolic adaptation as mentioned above. Lifespan is increased under caloric restriction and in Caenorhabditis elegans, the AMPK ortholog is necessary for this.

In summary, AMPK activation triggers beneficial effects on nutrient metabolism and inflammation and mimics the effect of insulin sensitizing changes in lifestyle. This pathway is still responsive in insulin resistant tissues and acts in an insulin-independent manner, indicating its relevance as potential drug target in metabolic disorders. In contrast, a worrisome effect of AMPK targeting is the increased appetite triggered by its activation in the brain [9]. However, as shown by AICAR treatment in insulinresistant rats, the overall effect of AMPK activation is still beneficial to metabolic disorders.

\section{The fine regulation of AMPK}

\section{Structural overview and kinase activation}

AMPK is a heterotrimeric kinase with a catalytic $(\alpha)$ and two regulatory ( $\beta$ and $\gamma$ ) subunits (Fig. 2). The $\alpha$-subunit carries the serine-threonine kinase domain, as well as an auto-inhibitory domain. The $\alpha$-subunit is tethered to the $\gamma$-subunit via a $\beta$-sheet, the 
core of which is made by the $\beta$-subunit. On top of this scaffolding role, the $\beta$-subunit carries a carbohydrate-binding module (CBM) that is able to bind to carbohydrates without exerting catalytic functions. The $\gamma$-subunit contains the sites for adenine nucleotide sensing. Each subunit exists in different isoforms $(\alpha 1, \alpha 2, \beta 1, \beta 2, \gamma 1, \gamma 2, \gamma 3)$, the location and role of which can differ across tissue and time (see Chapter 2). [9]

AMPK activity is increased 100 -fold by phosphorylation of the Thr172 residue of the $\alpha$-subunit (numbering of human $\alpha 2$, equivalent to Thr183 in $\alpha 1$ ) by either of the three AMPK kinases: liver kinase B1 (LKB1), calmodulin-dependent protein kinase kinase $\beta$ $(\mathrm{CaMKK} \beta)$ or transforming growth factor $\beta$-activated protein kinase 1 (TAK1) [13]. LKB1, often considered the most prominent AMPK kinase in many tissues [11], is constitutively active and its action on AMPK is mostly regulated by subcellular localization and AMPK status (see below). CaMKK $\beta$ is triggered by calcium accumulation while TAK1 relevant cases of AMPK regulation still require further investigation.

The precise phosphatases responsible for removal of the Thr172 phosphorylation are less well-established than the AMPK kinases. In the pancreatic cell line MIN6 $\beta$, the protein phosphatase 1 (PP1) was found to have an important role and to require the targeting subunit R6 to act on AMPK [20]. At least two other phosphatases, PP2A and PP2C, are also able to dephosphorylate AMPK, although little is known about how this is regulated [9]. Recently, an additional phosphatase linked to AMPK binding to ATP has been unveiled with $\alpha$-SNAP [21].

The mechanism that gave its name to the kinase is the activation of AMPK by AMP. Specifically, when cellular stresses such as hypoxia, caloric restriction or exercise occur, ATP is consumed and converted into ADP while the catabolic reactions cannot manage to compensate this conversion. In an effort to preserve cellular energy, the adenylate kinase converts two ADP molecules into an AMP and an ATP, leading to an accumulation of AMP $[8,22]$. The $\gamma$-subunit of AMPK with its four repeats of cystathionine $\beta$-synthase (CBS) can theoretically accommodate four adenine nucleotides in total, but site 2 is unable to bind nucleotide due to the absence of a key residue. Using X-ray crystallography, the sites 1 and 4 were found occupied by either AMP or ATP and the site 3 was only seen binding AMP or unoccupied [23]. The ability to bind both adenine nucleotides makes AMPK a sensor of the AMP/ATP ratio (Fig. 2): although the concentration of ATP is at all times much higher than that of AMP, the affinity plays in favor of AMP binding and a slight change in adenine nucleotide ratios lead to an exchange of nucleotide [22]. The effect of this change toward AMP binding is threefold: promotion of Thr172 phosphorylation by AMPK kinases, protection from dephosphorylation of the same site, and direct allosteric activation.

ADP was also shown to trigger both protection and increased Thr172 phosphorylation in vitro [22]. However, the relevance of this binding on modulating AMPK activity in 
vivo is questionable: although ADP concentration is 10 -fold higher than that of AMP and the nucleotide might therefore be more likely to bind, the affinity of AMPK for ADP is lower, which reduces the chances of binding [8].

The sensing of AMP by the $\gamma$-subunit is transmitted to the catalytic $\alpha$-subunit via the $\alpha$-RIM ( $\alpha$-regulatory subunit interacting motif), a flexible loop extending from the $\alpha$-subunit which makes contact with the $\gamma$-subunit directly at the interface with the nucleotides (Fig. 2) [24]. Indeed, the phosphor chain of the nucleotides all point in the same solvent exposed direction but the binding of ATP gives a radically different conformation to the $\gamma$-subunit than that of its AMP-bound state [23]. This change is sensed by the $\alpha$-RIM and passed on to the kinase domain. Regulation by posttranslational modification and subcellular localization

Apart from the Thr172 phosphorylation, which activates AMPK, many posttranslational modifications regulate AMPK activity, either directly or by changing the subcellular localization and thus the available downstream targets.

Among the activity-regulating mechanisms, mTOR-p70S6 kinase, downstream of Akt, phosphorylates AMPK on Ser485/491, which inhibits Thr172 phosphorylation, thereby defining a crosstalk between the insulin and the energy-sensing pathways [25, 26]. Autophosphorylation of AMPK also plays an important role in the regulation of its activity. For instance the site of Ser108 on the $\beta$-subunit allows for the kinase domain of the $\alpha$-subunit and the CBM to interact and form a cavity where one of the known activators of AMPK, A-769662 binds [27]. Interestingly, mutation of Ser108 to nonphosphorylatable alanine decreases basal AMPK activity [28], which suggests that this site plays a role in AMPK regulation even in absence of the pharmacological compounds. The $\beta$-subunit of AMPK may also be myristoylated, which is capital for full activation of AMPK and subcellular localization [28]. Indeed, upon AMP binding, AMPK is recruited by the AXIN scaffold enzyme, in complex with LKB1, and targeted to the lysosome membrane [29], which explains how AMP can trigger AMPK Thr172 phosphorylation. This reaction is dependent on the presence of a myristoyl group on the N-term of the $\beta$-subunit. Other modifications such as O-linked $\beta$ - $N$-acetylglucosamine [30] and sumoylation on the $\beta$-subunit also impact AMPKactivity, while its ubiquitination affects protein stability [31].

Beyond the membrane binding triggered by myristoylation, AMPK exists in different cellular compartments but little is known about the associated regulation. Autophosphorylation of the $\beta$-subunit at Ser24/25 or phosphorylation by an unknown kinase at Ser182 trigger AMPK nuclear export [28]. How or if this relates to the nuclear export signal identified on the $\alpha$-subunit has not been addressed yet [32]. Therefore, AMPK is a highly regulated kinase, and more information is needed before a full structure-activity relationship can be given for this enzyme. 


\section{Interdependence between AMPK and glycogen}

Glycogen is the principal storage form of carbohydrates in mammals and is made of successions of glucose units bound in $\alpha-1,4$ orientation and regular branch points with $\alpha-1,6$ linkage [33]. Cellular glycogen granules contain not only carbohydrates but also a great number of proteins. Among them are (i) the proteins responsible for glycogen synthesis (glycogenin which branches the first glucose units, glycogen synthase which catalyzes the following $\alpha-1,4$ linkage and the branching enzyme for the $\alpha-1,6$ linkages) and (ii) the proteins required for the degradation of glycogen (the glycogen phosphorylase and debranching enzyme, respectively, for $\alpha-1,4$ and $\alpha-1,6$ linkages) [33]. Other glycogen-associated proteins often have a role in glycogen regulation: for instance, the aforementioned protein phosphatase 1 regulating subunits $\left(R_{G L}, G_{M}, R 6\right.$, etc.) bind glycogen and target the phosphatase activity to dephosphorylate glycogen synthase [34].

Different systems exist to allow this binding. Some proteins such as glycogen synthase rely on the existence of multiple binding pockets associated with the catalytic site [33]. Other proteins such as R6 developed self-folding domains, the CBM, to allow attachment without exerting catalytic activity [34,35]. As mentioned above, AMPK belongs to this second group, with a domain from the CBM48 family in its $\beta$-subunit [36], and all known homologous $\beta$-subunits also show a similar $N$-isoamylase domain although not all can actually bind carbohydrates [37]. Mammalian AMPK, however, binds to glycogen and other oligosaccharides in vitro via the CBM and, when overexpressed in cells, co-localizes with an anti-glycogen antibody [37, 38].

Strikingly, many reports draw a link between AMPK and glycogen although the complete interplay is still unclear to date. In McArdle disease patients, a mutation of glycogen phosphorylase induces high levels of glycogen, and in patient cells, AMPK is overactivated [9]. Similarly, a residue mutation in the $\gamma$-subunit of AMPK renders it insensitive to adenine nucleotides leading to a dysregulated and yet increased activity of AMPK, as well as an abnormally high glycogen level in the heart (as found in a human inherited disease called Wolf-Parkinson-White syndrome) or in the skeletal muscle (as reported in Hampshire pigs) [14]. In contrast, the effects of AICAR and contraction on glucose uptake are downregulated in muscle containing high levels of glycogen [39, 40]. Furthermore, the reported effect of AMPK on glycogen synthesis is variable: according to some reports, AMPK inhibits synthesis by phosphorylation of glycogen synthase [41], whereas others found this inhibition is overridden by AMPK-induced glucose uptake that then allosterically activates glycogen synthase [42]. In accord with this, long term AICAR treatment leads to increased glycogen synthesis [43]. Therefore, although a clear link exists between AMPK and glycogen, knowledge about their 
interplay is still evolving and ia as yet incomplete.

Since AMPK activity was firstly reported as being unaffected by glycogen binding, the glycogen association was thought to influence only its subcellular localization [38, 44]. As AMPK is known to interact and phosphorylate glycogen-bound targets, colocalization with its downstream targets as conferred by the CBM would allow for quick initiation of the phosphorylation cascade $[38,41]$. Later, however, it turned out that AMPK activity was inhibited by glycogen binding when a branching point is exposed, i.e. when glycogen degradation has started [45]. Interestingly, the best AMPKCBM ligands among oligosaccharide share a $\alpha-1,6$ branching [46], suggesting that AMPK interaction with glycogen truly is regulated by glycogen branching levels.

In summary, the reciprocal regulation between AMPK activity and glycogen metabolism requires further investigation but the CBM of AMPK clearly impact on AMPK activity, notably by defining different pools of AMPK either bound to glycogen or not $[9,41]$. Interestingly, diabetic patients have a disturbed glycogen metabolism due to impaired insulin signaling [1], but how this impacts on AMPK localization or activity is unknown.

\section{Drug design process: application to AMPK in metabolic diseases}

For centuries, pharmacologic advances depended on empirical discoveries based on the use of natural products, as for instance salicylate extracted from willow bark, which is used by man since thousands of years and is the base to today's Aspirin [32]. The mode of action of many of these ancient drugs remains to some extent a black box even nowadays, as they generally target a wide set of proteins. Salicylate, for instance, was established to be a direct activator of AMPK only in 2012 [47], and the drug Aspirin, after degradation into salicylic acid, may be acting on inflammation notably via AMPK [32]. Since these ancient times however, advances in chemistry allowed for the large scale synthesis of molecules and gave birth to pharmaceutical industries [48]. Discoveries are no longer left to serendipity and follow an established pipeline from target identification to market introduction.

\section{From target identification to hit}

Because drug development processes often fail due to lack of efficiency or safety, target identification and validation are critical steps [49]. In this stage, a strong link between the chosen target and the studied disease has to be proven to ensure efficiency and considerable knowledge needs to be available to predict safety [50]. Information from disease-related expression profiles, disease-causing polymorphisms or genetic models, can be used to assess whether the targeting could be beneficial for the course of a disease, and whether this targeting can be safe for the patient. The many omics 
data available today come in handy in this stage although powerful bioinformatics tools are required to integrate and efficiently mine the great amount of data that are generally available [49].

Once the target is identified and further confirmed, for instance by additional genomic or antibody targeting, the next challenge is the development of an assay that is highly sensitive to distinguish inactive from active molecules. The molecule in question can be a small drug-like chemical compound, an antibody or another class of biomolecule such as a peptide: the definition of the target and its location often motivates the choice of targeting strategy [50]. Screening requires the availability of a library of chemicals or, for instance, phage display to provide a set of specific antibodies and peptides. Numerous screening assay types exist: biochemical ones where the purified protein of interest is tested, for instance, with nuclear magnetic resonance (NMR) or with direct activity measurements, cell-based ones where engineered cell lines overexpressing the protein of interest are monitored at the single-cell level, or even tissue-based ones for a more physiological output [50]. Of course, depending on the assay type, different sizes of library can be screened: the more complex assays require targeted libraries based on knowledge of the target, while a simpler assay allows for high-throughput screening where millions of molecules can be screened in a matter of weeks. A method to downsize and prioritize the screened library is virtual ligand screening (VLS) whereby the 3D structure of the target (structure based VLS) or of known binders (ligand based VLS) allows to define families of compounds or key characteristics for potential binders [51]. In all assays and screening steps, reproducibility and data quality are highly important factors in setting up the assay: these criteria can notably be tested with training sets (if available). The output of the screening is the identification of hits, i.e. compounds with a desired activity towards the target [52].

\section{From hit to lead and onward}

For chemical compounds, an important factor to take into consideration is the ADME/tox profile (absorption, distribution, metabolism, excretion, toxicity), which defines the ability of the compound to act as a drug [52]. This ability can partly be predicted according to filters such as the rule of five from Lipinski, which states that compounds with a molecular weight $<500 \mathrm{Da}$, hydrophobicity of $\log \mathrm{P}<5$, hydrogen bonds donors $<5$ and hydrogen bond acceptors $<10$ are similar to known drugs and therefore are more likely to behave as such in the body [53]. Screened libraries are often filtered according to these criteria beforehand, but the optimization phase following the hit discovery is also the time where these properties can be refined by direct assessment. In that stage, the hits obtained after primary screening are further 
tested to obtain dose dependent response curves and are clustered according to their structure [50]. This double information is used to start drawing structure-activity relationships and predicting both active and inactive compounds to refine the picture. Once families of hits have been well defined, one or preferably several of them are carried on in the hit-to-lead process which will require the molecules to meet much more stringent standards. In this stage, deepened structure activity relationship studies are done and the compounds are optimized simultaneously for efficacy, selectivity and pharmacokinetic properties [52]. On top of the previously used assays, new cellular and biochemical assays are applied to test selectivity and X-ray crystallography or NMR is used to assess the precise binding mode on the target. Animal disease models are used to further test the compounds in a more physiological context, notably according to pharmacokinetic and toxicological criteria. Even once a compound has undergone sufficient characterization to become a pre-clinical candidate, the discovery team will keep on testing molecules in case a back-up would be needed.

Only $10 \%$ of all small molecule projects reach the pre-clinical stage, and for each of them, only a couple of molecules out of hundreds tested will carry on in that stage. Although many precautions are taken during this development process, leading to this high attrition rate, one molecule in ten successfully passing the pre-clinical step will ever reach the market [50]. Even after pre-clinical approbation, many stages of clinical studies remain ahead with increasing degrees of requirements. Due to this, many compounds fail even at an advanced stage and back-up compounds will need to go over the same steps again, explaining the current cost of the drug development process of more than one billion U.S. dollars per marketed compound $[49,50]$.

\section{A well-defined target for metabolic disorders: AMPK}

As outlined above, AMPK activation is already firmly established as beneficial for metabolic disorders: the effect on nutrient uptake and catabolism as well as on inflammation makes it a validated target for reestablishment of the metabolic balance $[11,13]$. A few direct AMPK activators have been synthesized in the past but none of them reached the market to date (see Chapter 2). However, several indirect activators are regularly used in humans. Indirect activators generally target the mitochondrial respiratory chain preventing the production of ATP and thus mimic the energy stress status found in exercising muscle or during caloric restriction [54]. Among these molecules is the well-established metformin, one of the most prescribed drugs in the world for its anti-hyperglycemic effect [55]. Metformin, similarly to phenformin from which it was derived, appears to bind directly to the membrane of mitochondria [56]. Through direct targeting of the respiratory chain, metformin has many effects which 
are partly unrelated to AMPK, as metformin was notably shown to switch off hepatic glucose output in the absence of AMPK presumably because the low levels of ATP directly prevented this energy consuming pathway to occur [56]. Still, metformininduced AMPK activation clearly plays a role in the beneficial outputs of the drug, such as increased fatty acid oxidation. Even if metformin can only point to but not prove the efficacy of AMPK targeting in metabolic disorders, it clearly established the safe effect of AMPK targeting. Similarly, several nutraceuticals such as resveratrol, curcumin or berberine, also act on AMPK via mitochondrial targeting to exert a beneficial effect, showing the relevance of AMPK targeting in nutrient induced disorders [8].

However, despite the efficiency of indirect AMPK activators, one can still wonder about the long-term consequences of compounds that basically poison mitochondria. In accord with this view, the search for direct AMPK activators that can be applied in the clinics and that probably are safer than those compounds that target mitochondrial function is still ongoing.

\section{Outline of the thesis}

Even though AMPK is clearly a relevant target in metabolic disorders, the previous targeting programs as yet failed to bring a compound to the market. In addition, many gaps of knowledge remain in both the metabolic disorder mechanisms and in AMPK regulation. Notably, the link between AMPK, glycogen and glycogen-bound proteins still requires further investigation. With this thesis, we intend to extend our knowledge about the interrelation between AMPK and glycogen in the context of glucose metabolism. In Chapter 2, the relevance of AMPK targeting is broadened to many other pathological conditions than the metabolic disorders. The possibility and status of AMPK isoform-specific targeting is discussed and new possible targeting options, in particular those that translate recent advances in AMPK structural knowledge, are described. Chapter 3 establishes a novel site in AMPK $\beta$ that is subject to AMPK autophosphorylation thereby affecting the subcellular localization, and specifically preventing AMPK binding to glycogen. The mutation of this newly identified site leads to changes in glycogen turnover regulation, suggesting an important role of AMPK carbohydrate association on glycogen regulation. The subject of Chapter 4 is to define the glucose-regulated interaction of AMPK with the protein phosphatase 1 targeting subunit R6: glucose deprivation lowers levels of glycogen and simultaneously increases the R6-AMPK interaction. The autophosphorylation site identified in Chapter 3 is strongly involved in this association. Chapter $\mathbf{5}$ describes a strategy to design small molecules capable of preventing the AMPK binding to carbohydrate, which combines a virtual ligand screening with two in vitro steps. The screening process was followed by further hit characterization and yielded the discovery of a novel AMPK activator with 
possible beneficial outcome in nutrient uptake. Finally, Chapter 6 provides with a general discussion of the newly identified mechanisms and the new compound in the context of metabolic disorders and beyond.

The experimental work executed for this thesis is part of a wider project funded by the VIDI-Innovational Research Grant scheme from the Netherlands Organization of Scientific Research (NWO-ALW 864.10.007; to D. Neumann). In particular, the studies presented here were performed in close collaboration with a second PhD candidate, Yvonne Oligschläger thus producing inevitable overlap in the experimental chapters of the two theses. 


\section{References}

1. Samuel, V.T. and Shulman, G.I., Mechanisms for insulin resistance: common threads and missing links. Cell, 2012. 148(5): p. 852-871.

2. DeBerardinis, R.J. and Thompson, C.B., Cellular metabolism and disease: what do metabolic outliers teach us? Cell, 2012. 148(6): p. 1132-1144.

3. Tsatsoulis, A., Mantzaris, M.D., Bellou, S. and Andrikoula, M., Insulin resistance: an adaptive mechanism becomes maladaptive in the current environment - an evolutionary perspective. Metabolism, 2013.62(5): p. 622-633.

4. Ashcroft, F.M. and Rorsman, P., Diabetes mellitus and the beta cell: the last ten years. Cell, 2012. 148(6): p. 1160-1171.

5. Boren, J., Taskinen, M.R., Olofsson, S.O. and Levin, M., Ectopic lipid storage and insulin resistance: a harmful relationship. J Intern Med, 2013. 274(1): p. 25-40.

6. Flamment, M., Hajduch, E., Ferre, P. and Foufelle, F., New insights into ER stress-induced insulin resistance. Trends Endocrinol Metab, 2012. 23(8): p. 381-390.

7. Crozet, P., Margalha, L., Confraria, A., Rodrigues, A., Martinho, C., Adamo, M., Elias, C.A. and Baena-Gonzalez, E., Mechanisms of regulation of SNF1/AMPK/SnRK1 protein kinases. Front Plant Sci, 2014. 5: p. 190.

8. Hardie, D.G., AMP-activated protein kinase: maintaining energy homeostasis at the cellular and whole-body levels. Annu Rev Nutr, 2014. 34: p. 31-55.

9. Viollet, B., Horman, S., Leclerc, J., Lantier, L., Foretz, M., Billaud, M., Giri, S. and Andreelli, F., AMPK inhibition in health and disease. Crit Rev Biochem Mol Biol, 2010. 45(4): p. 276-295.

10. Hardie, D.G., Ross, F.A. and Hawley, S.A., AMPK: $a$ nutrient and energy sensor that maintains energy homeostasis. Nat Rev Mol Cell Biol, 2012. 13(4): p. 251-262.

11. Hegarty, B.D., Turner, N., Cooney, G.J. and Kraegen, E.W., Insulin resistance and fuel homeostasis: the role of AMP-activated protein kinase. Acta Physiol (Oxf), 2009. 196(1): p. 129-145.

12. Mihaylova, M.M. and Shaw, R.J., The AMPK signalling pathway coordinates cell growth, autophagy and metabolism. Nat Cell Biol, 2011. 13(9): p. 1016-1023.

13. Fogarty, S. and Hardie, D.G., Development of protein kinase activators: AMPK as a target in metabolic disorders and cancer. Biochim Biophys Acta, 2010. 1804(3): p. 581-591.

14. Viollet, B., Athea, Y., Mounier, R., Guigas, B., Zarrinpashneh, E., Horman, S., Lantier, L., Hebrard, S., Devin-Leclerc, J., Beauloye, C., Foretz, M., Andreelli, F., Ventura-Clapier, R. and Bertrand, L., AMPK: Lessons from transgenic and knockout animals. Frontiers in bioscience, 2009. 14: p. 19-44.
15. Saltiel, A.R. and Kahn, C.R., Insulin signalling and the regulation of glucose and lipid metabolism. Nature, 2001. 414(6865): p. 799-806.

16. Luiken, J.J., Dyck, D.J., Han, X.X., Tandon, N.N., Arumugam, Y., Glatz, J.F. and Bonen, A., Insulin induces the translocation of the fatty acid transporter FAT/CD36 to the plasma membrane. Am J Physiol Endocrinol Metab, 2002. 282(2): p. E491-495.

17. Burkewitz, K., Zhang, Y. and Mair, W.B., AMPKat the nexus of energetics and aging. Cell Metab, 2014. 20(1): p. 10-25.

18. Xiao, B., Sanders, M.J., Carmena, D., Bright, N.J., Haire, L.F., Underwood, E., Patel, B.R., Heath, R.B., Walker, P.A., Hallen, S., Giordanetto, F., Martin, S.R., Carling, D. and Gamblin, S.J., Structural basis of AMPK regulation by small molecule activators. Nat Commun, 2013. 4: p. 3017.

19. Li, X., Wang, L., Zhou, X.E., Ke, J., de Waal, P.W., Gu, X., Tan, M.H., Wang, D., Wu, D., Xu, H.E. and Melcher, K., Structural basis of AMPK regulation by adenine nucleotides and glycogen. Cell research, 2015. 25(1): p. 50-66.

20. Garcia-Haro, L., Garcia-Gimeno, M.A., Neumann, D., Beullens, M., Bollen, M. and Sanz, P., The PP1R6 protein phosphatase holoenzyme is involved in the glucose-induced dephosphorylation and inactivation of AMP-activated protein kinase, a key regulator of insulin secretion, in MIN6 beta cells. FASEB J, 2010. 24(12): p. 5080-5091.

21. Wang, L. and Brautigan, D.L., alpha-SNAP inhibits AMPK signaling to reduce mitochondrial biogenesis and dephosphorylates Thr172 in AMPKalpha in vitro. Nat Commun, 2013. 4: p. 1559.

22. Hardie, D.G., Energy sensing by the AMP-activated protein kinase and its effects on muscle metabolism. The Proceedings of the Nutrition Society, 2011. 70(1): p. 92-99.

23. Chen, L., Wang, J., Zhang, Y.Y., Yan, S.F., Neumann, D., Schlattner, U., Wang, Z.X. and Wu, J.W., AMP-activated protein kinase undergoes nucleotide-dependent conformational changes. Nat Struct Mol Biol, 2012. 19(7): p. 716-718.

24. Chen, L., Xin, F.J., Wang, J., Hu, J., Zhang, Y.Y., Wan, S., Cao, L.S., Lu, C., Li, P., Yan, S.F., Neumann, D., Schlattner, U., Xia, B., Wang, Z.X. and Wu, J.W., Conserved regulatory elements in AMPK. Nature, 2013. 498(7453): p. E8-10.

25. Dagon, Y., Hur, E., Zheng, B., Wellenstein, K., Cantley, L.C. and Kahn, B.B., p70S6 kinase phosphorylates $A M P K$ on serine 491 to mediate leptin's effect on food intake. Cell Metab, 2012. 16(1): p. 104-112.

26. Hurley, R.L., Barre, L.K., Wood, S.D., Anderson, K.A., Kemp, B.E., Means, A.R. and Witters, L.A., Regulation of AMP-activated protein kinase by multisite phosphorylation in response to agents that elevate cellular cAMP. J Biol Chem, 2006. 281(48): p. 36662-36672. 
27. Sanders, M.J., Ali, Z.S., Hegarty, B.D., Heath, R., Snowden, M.A. and Carling, D., Defining the mechanism of activation of AMP-activated protein kinase by the small molecule A-769662, a member of the thienopyridone family. J Biol Chem, 2007. 282(45): p. 32539-32548.

28. Warden, S.M., Richardson, C., O’Donnell, J., Jr., Stapleton, D., Kemp, B.E. and Witters, L.A., Posttranslational modifications of the beta-1 subunit of AMP-activated protein kinase affect enzyme activity and cellular localization. Biochem J, 2001. 354(Pt 2): p. 275-283.

29. Zhang, Y.L., Guo, H., Zhang, C.S., Lin, S.Y., Yin, Z., Peng, Y., Luo, H., Shi, Y., Lian, G., Zhang, C., Li, M., Ye, Z., Ye, J., Han, J., Li, P., Wu, J.W. and Lin, S.C., AMP as a low-energy charge signal autonomously initiates assembly of AXIN-AMPK-LKB1 complex for AMPK activation. Cell Metab, 2013. 18(4): p. 546-555.

30. Bullen, J.W., Balsbaugh, J.L., Chanda, D., Shabanowitz, J., Hunt, D.F., Neumann, D. and Hart, G.W., Cross-talk between two essential nutrientsensitive enzymes: O-GlcNAc transferase (OGT) and $A M P$-activated protein kinase (AMPK). J Biol Chem, 2014. 289(15): p. 10592-10606.

31. Sanz, P., Rubio, T. and Garcia-Gimeno, M.A., AMPKbeta subunits: more than just a scaffold in the formation of AMPK complex. FEBS J, 2013. 280(16): p. 3723-3733.

32. Hardie, D.G., AMPK--sensing energy while talking to other signaling pathways. Cell Metab, 2014. 20(6): p. 939-952.

33. Roach, P.J., Depaoli-Roach, A.A., Hurley, T.D. and Tagliabracci, V.S., Glycogen and its metabolism: some new developments and old themes. Biochem J, 2012. 441(3): p. 763-787.

34. Graham, T.E., Yuan, Z., Hill, A.K. and Wilson, R.J., The regulation of muscle glycogen: the granule and its proteins. Acta Physiol (Oxf), 2010. 199(4): p. 489-498.

35. Shoseyov, O., Shani, Z. and Levy, I., Carbohydrate binding modules: biochemical properties and novel applications. Microbiol Mol Biol Rev, 2006. 70(2): p. 283-295.

36. Janecek, S., Svensson, B. and MacGregor, E.A., Structural and evolutionary aspects of two families of non-catalytic domains present in starch and glycogen binding proteins from microbes, plants and animals. Enzyme Microb Technol, 2011. 49(5): p. 429-440.

37. Polekhina, G., Gupta, A., Michell, B.J., van Denderen, B., Murthy, S., Feil, S.C., Jennings, I.G., Campbell, D.J., Witters, L.A., Parker, M.W., Kemp, B.E. and Stapleton, D., AMPK beta subunit targets metabolic stress sensing to glycogen. Current biology : CB, 2003. 13(10): p. 867-871.

38. Hudson, E.R., Pan, D.A., James, J., Lucocq, J.M., Hawley, S.A., Green, K.A., Baba, O., Terashima, T. and Hardie, D.G., A novel domain in AMP-activated protein kinase causes glycogen storage bodies similar to those seen in hereditary cardiac arrhythmias. Current biology : CB, 2003. 13(10): p. 861-866.
39. Nielsen, J.N., Derave, W., Kristiansen, S., Ralston, E., Ploug, T. and Richter, E.A., Glycogen synthase localization and activity in rat skeletal muscle is strongly dependent on glycogen content. J Physiol, 2001. 531(Pt 3): p. 757-769.

40. Wojtaszewski, J.F., Jorgensen, S.B., Hellsten, Y., Hardie, D.G. and Richter, E.A., Glycogen-dependent effects of 5-aminoimidazole-4-carboxamide (AICA)-riboside on AMP-activated protein kinase and glycogen synthase activities in rat skeletal muscle. Diabetes, 2002. 51(2): p. 284-292.

41. Jorgensen, S.B., Nielsen, J.N., Birk, J.B., Olsen, G.S., Viollet, B., Andreelli, F., Schjerling, P., Vaulont, S., Hardie, D.G., Hansen, B.F., Richter, E.A. and Wojtaszewski, J.F., The alpha2-5'AMP-activated protein kinase is a site 2 glycogen synthase kinase in skeletal muscle and is responsive to glucose loading. Diabetes, 2004. 53(12): p. 3074 3081.

42. Hunter, R.W., Treebak, J.T., Wojtaszewski, J.F. and Sakamoto, K., Molecular mechanism by which AMP-activated protein kinase activation promotes glycogen accumulation in muscle. Diabetes, 2011. 60(3): p. 766-774.

43. Holmes, B.F., Kurth-Kraczek, E.J. and Winder, W.W., Chronic activation of 5'-AMP-activated protein kinase increases GLUT-4, hexokinase, and glycogen in muscle. J Appl Physiol (1985), 1999. 87(5): p. 1990-1995.

44. Polekhina, G., Gupta, A., van Denderen, B.J., Feil, S.C., Kemp, B.E., Stapleton, D. and Parker, M.W., Structural basis for glycogen recognition by AMP-activated protein kinase. Structure, 2005. 13(10): p. 1453-1462.

45. McBride, A., Ghilagaber, S., Nikolaev, A. and Hardie, D.G., The glycogen-binding domain on the AMPK beta subunit allows the kinase to act as a glycogen sensor. Cell Metab, 2009. 9(1): p. 2334.

46. Koay, A., Woodcroft, B., Petrie, E.J., Yue, H., Emanuelle, S., Bieri, M., Bailey, M.F., Hargreaves, M., Park, J.T., Park, K.H., Ralph, S., Neumann, D., Stapleton, D. and Gooley, P.R., AMPK beta subunits display isoform specific affinities for carbohydrates. FEBS Lett, 2010. 584(15): p. 3499 3503.

47. Hawley, S.A., Fullerton, M.D., Ross, F.A., Schertzer, J.D., Chevtzoff, C., Walker, K.J., Peggie, M.W., Zibrova, D., Green, K.A., Mustard, K.J., Kemp, B.E., Sakamoto, K., Steinberg, G.R. and Hardie, D.G., The ancient drug salicylate directly activates AMP-activated protein kinase. Science, 2012. 336(6083): p. 918-922.

48. Drews, J., Drug discovery: a historical perspective. Science, 2000. 287(5460): p. 1960-1964.

49. Smith, C., Drug target validation: Hitting the target. Nature, 2003. 422(6929): p. 341, 343, 345 passim.

50. Hughes, J.P., Rees, S., Kalindjian, S.B. and Philpott, K.L., Principles of early drug discovery. Br J Pharmacol, 2011. 162(6): p. 1239-1249.

51. Phatak, S.S., Stephan, C.C. and Cavasotto, C.N., High-throughput and in silico screenings in drug 
discovery. Expert opinion on drug discovery, 2009. 4(9): p. 947-959.

52. Hughes, M., Inglese, J., Kurtz, A., Andalibi, A., Patton, L., Austin, C., Baltezor, M., Beckloff, M., Sittampalam, S., Weingarten, M. and Weir, S., Early Drug Discovery and Development Guidelines: For Academic Researchers, Collaborators, and Start-up Companies, in Assay Guidance Manual, G.S. Sittampalam, et al., Editors. 2004: Bethesda (MD).

53. Pollastri, M.P., Overview on the Rule of Five. Curr Protoc Pharmacol, 2010. Chapter 9: p. Unit 9 12.
54. Rana, S., Blowers, E.C. and Natarajan, A., Small molecule adenosine 5'-monophosphate activated protein kinase (AMPK) modulators and human diseases. J Med Chem, 2015. 58(1): p. 2-29.

55. Wang, S., Song, P. and Zou, M.H., AMP-activated protein kinase, stress responses and cardiovascular diseases. Clin Sci (Lond), 2012. 122(12): p. 555-573.

56. Foretz, M., Guigas, B., Bertrand, L., Pollak, M. and Viollet, B., Metformin: from mechanisms of action to therapies. Cell Metab, 2014. 20(6): p. 953-966. 



\section{Chapter 2}

\section{Novel targeting opportunities for AMP-activated protein kinase: isoform specificity and computer- aided drug design}

Marie Miglianico, Gerry A.F. Nicolaes, Dietbert Neumann

In revision (invited in J Med Chem) 


\begin{abstract}
As a central regulator of metabolism, the AMP-activated protein kinase (AMPK) is an established therapeutic target for metabolic diseases. Beyond the metabolic area, the number of medical fields that involve AMPK grows continuously, expanding the potential applications for AMPK modulators. Even though indirect AMPK activators are used in the clinics for their beneficial metabolic outcome, the few described direct agonists all failed to reach the market to date, which leaves options open for novel targeting methods. As AMPK is not actually a single molecule and has different roles depending on its isoform composition, the opportunity for isoform-specific targeting has notably come forward, but the currently available modulators fall short of expectations. In this review, we argue that with the amount of available structural and ligand data, computer-based drug design offers a number of opportunities to undertake novel and isoform-specific targeting of AMPK.
\end{abstract}




\section{Introduction}

From the unicellular organism to the complex metazoans, monitoring energy availability is capital to maintain life in a dynamic environment [1]. In mammals, this role is ensured by the AMP-activated protein kinase (AMPK) which gets activated during energy deficiency and acts to restore the metabolic balance [1, 2]. Upon cellular stress, for instance a drop in ATP levels, AMPK triggers the necessary metabolic adaptation by downregulation of energy-consuming, anabolic pathways and upregulation of catabolism. During fast for instance, AMPK is an important component of the metabolic flexibility, which allows the switch from carbohydrate to high energy lipid consumption in muscle [3].

Many diseases originate from a maladaptation to changing conditions. A most prominent example in human, type 2 diabetes, results from the incapacity of our body to deal with a continuous energy surplus [4]. In the case of cancer, tumor cells override the adaptive controls that coordinate tissue life and grow at the expense of the rest of the body [5]. In both cases and in many more, AMPK was shown to be implicated, for better or worse, and its modulation presents an opportunity for therapeutic intervention.

A great amount of knowledge about AMPK function and regulation has been accumulated over the years and brought the identification of several AMPK modulators [6]. But so far, a safe targeting of the kinase applicable to the clinics still eludes the community. An interesting, albeit challenging, option in the AMPK field is to target AMPK not as a single molecule but to take into account the different existing isoforms. Indeed, the increasing knowledge about the roles of AMPK isoforms points towards possible therapeutic applications for targeting of a specific subset of AMPK molecules.

In this perspective, after shortly presenting the regulation of the kinase and its implication as a signaling hub in many diseases, we discuss the feasibility and interest of having isoform specific targeting carried out, and finally suggest new drug-design strategies based on recent discoveries on AMPK regulation and structure.

\section{AMPK, a highly regulated protein kinase involved in an expanding number of diseases}

As a real signaling hub, AMPK is a protein kinase with several levels of regulation which is involved in a wide number of pathways. This primordial involvement in cellular regulation makes AMPK a potential therapeutic target in an ever-growing number of diseases. Interestingly, not only activation but also inhibition could find applications in different disorders. 


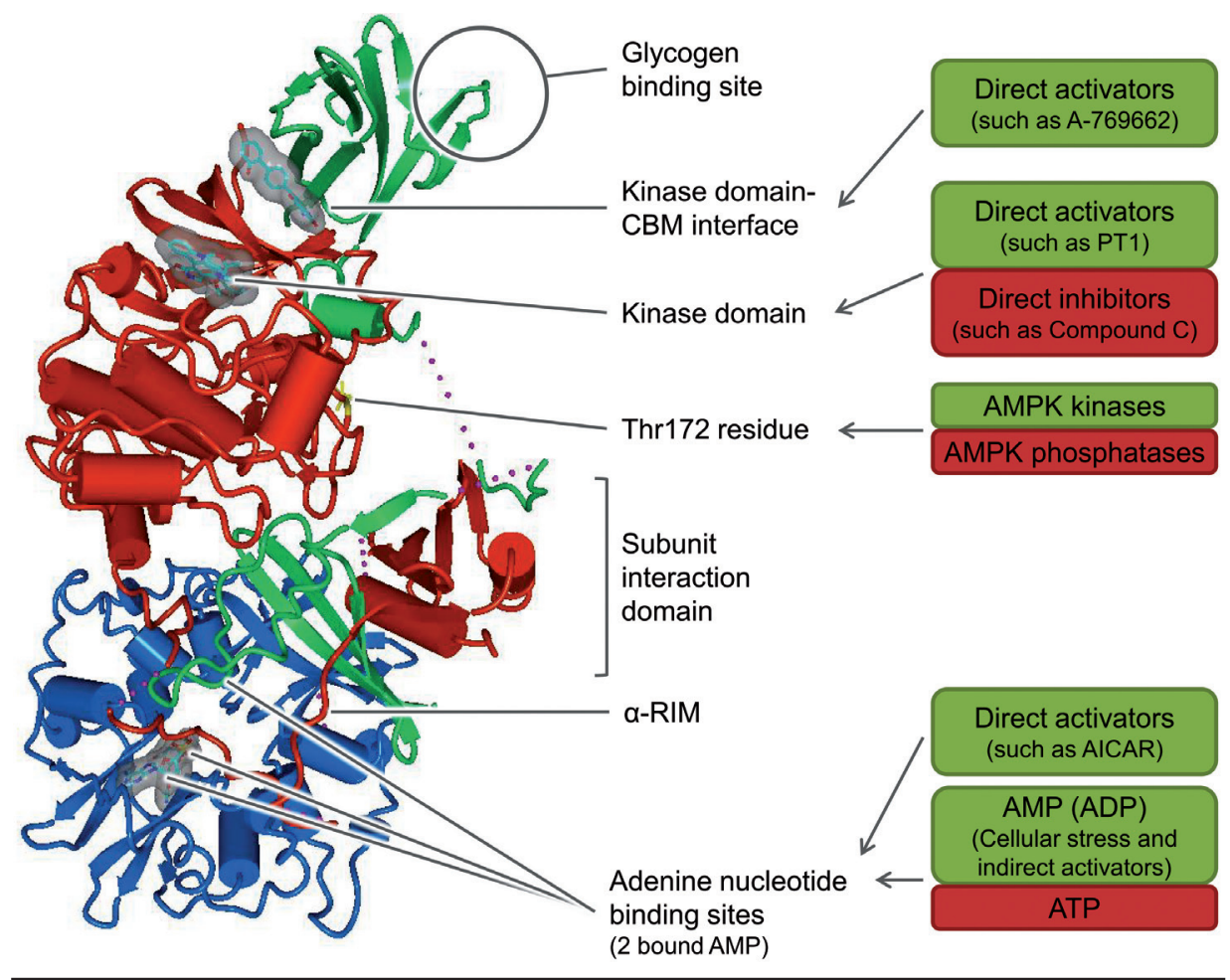

Figure 1. Overview of AMPK structure and regulation.

On the right, cartoon representation of AMPK structure (PDB ID: 4CFF, [7]) bound to A-769662 in the kinase domain-CBM interface, staurosporin in the active site and 2 AMP in sites 3 and 4 of the $\gamma$-subunit. The $\alpha, \beta$, and $\gamma$-subunits are represented in red, green and blue respectively. The middle panel highlights the important structural regions, while the right panel offers an overview of AMPK regulation. The green boxes refer to activating mechanisms, while the red ones are for inhibition. They are placed near the region they interfere with.

\section{Overview of AMPK structure}

AMPK is a heterotrimeric kinase composed of three subunits named $\alpha, \beta$, and $\gamma$ that exist in different isoforms $(\alpha 1, \alpha 2 ; \beta 1, \beta 2 ; \gamma 1, \gamma 2, \gamma 3)$ of overall similar structure (Fig. 1). At the $\mathrm{N}$-terminus of the catalytic $\alpha$-subunit, the serine-threonine kinase domain is classically constituted of two lobes which surround the active site and of an activation loop which is phosphorylated at residue Thr172 as a major activation step [8]. Next to the kinase domain, an auto-inhibitory domain maintains the kinase in an inactive form under basal conditions. Between this domain and the C-terminal end, an extended loop, termed the $\alpha$-regulatory subunit interacting motif ( $\alpha$-RIM), is in contact with the $\gamma$-subunit and is of capital importance for adenine nucleotide sensing [9].

In the regulatory $\beta$-subunit, a self-folding domain named carbohydrate-binding module (CBM) binds glycogen in vitro and in cells without exerting any catalytic activity 
on saccharides $[10,11]$. At the $C$-terminal end of the $\beta$-subunit, a $\beta$-sheet makes a scaffold that tethers $\alpha$ and $\gamma$-subunits together $[12,13]$ : the involved regions are hereafter called subunit interaction domains.

The regulatory $\gamma$-subunit contains four tandem cystathionine $\beta$-synthase (CBS) repeats which form four potential adenine nucleotide binding sites, of which three (sites 1, 3, and 4) are actually used [12]. Adenine nucleotide binding to these sites induces a conformational change which is transmitted via the $\alpha$-RIM to the kinase domain thereby directly influencing AMPK activity [9].

The AMPK trimer is therefore by itself a complex structure. The intrinsic regulation by adenine nucleotide and glycogen will be further explained in the section below and put in perspective with the more intricate general kinase regulation.

\section{Regulation of AMPK by different effectors and proteins}

Many intracellular and extracellular signaling pathways, generally related to cellular stress, converge toward AMPK signaling. Interestingly, the involvement of AMPK in these pathways is not only mediated by protein signaling as several cellular effectors are known to directly act as messenger and regulate the kinase.

As touched upon in the previous section, AMPK is a direct sensor of adenine nucleotide ratios $[1,2]$. Upon metabolic challenges such as hypoxia or exercise, ATP is consumed faster than it is produced which increases the level of ADP directly and of AMP indirectly through the intervention of the adenylate kinase [2]. This increase in ADP and AMP concentrations leads to their binding to the adenine nucleotide sensing sites of AMPK, which replaces the previously bound ATP. A replacement with AMP results in direct allosteric kinase activation, facilitates the Thr172 activating phosphorylation and protects from dephosphorylation of this same site. A change toward ADP has the same effect on AMPK phosphorylation as does the AMP replacement, except for the allosteric effect. However, the effect of AMP on AMPK seems more physiologically relevant than that of ADP due to the different affinities and concentrations of the two nucleotides [2].

Reactive oxygen and nitrogen species (ROS and RNS respectively) accumulation is another sign of cellular stress [14]. One study by Zmijewski et al. shows a direct activation of AMPK by $\mathrm{H}_{2} \mathrm{O}_{2}$ through oxidation of key cysteine residues in the $\alpha$-subunit [15], which suggests a direct sensing of oxidative stress by AMPK. However, this direct effect still remains to be confirmed as RNS production was also shown to activate AMPK via a protein kinase $\mathrm{C}$ and ROS production is sometimes associated with adenine nucleotide changes [14].

Another intracellular molecule that influences AMPK activity is glycogen. Even though the inter-regulation of AMPK and glycogen is not fully understood in vivo [1], 
AMPK is directly inhibited in vitro by glycogen binding via the CBM of the $\beta$-subunit [16]. Specifically, even though AMPK can bind many types of oligosaccharides, the carbohydrate molecules that simulate glycogen branching points potently inhibit AMPK activity, suggesting that glycogen branching level and not glycogen amount influences AMPK activity. As a possible explanation for this inhibition, carbohydrate binding by the CBM of AMPK is thought to disrupt the interaction between the CBM and the kinase domain which normally stabilizes the kinase in an active form [17]. Of note, the inhibition of AMPK by glycogen stays a controversial topic because one of the first studies that disclosed the role of the CBM reported no inhibition of AMPK by glycogen [11].

Therefore AMPK is directly regulated by adenine nucleotide, glycogen, and presumably oxidative stress. Additionally, calcium activates the calmodulin-dependent protein kinase kinase $\beta$ (CaMKK $\beta$ ) which in turn phosphorylates AMPK at the Thr172 site. Thus, calcium concentration could be seen as an additional molecular effector acting on AMPK even though the effect is indirect.

Aside from CaMKK $\beta$, the liver kinase B1 (LKB1) and the transforming growth factor $\beta$-activated protein kinase (TAK1) are two other identified AMPK kinases which can phosphorylate AMPK at Thr172. LKB1 is considered to be constitutively active and is often described as the main kinase for AMPK regulation [18]. Even though the conformational change induced by AMP (ADP) binding facilitates the access to Thr172 to all AMPK kinases, LKB1 action on AMPK was reported to have an additional level of AMP regulation. Indeed AMPK nucleotide binding triggers the formation of a complex with the scaffold protein AXIN and LKB1 on the membrane of the lysosome, where a crosstalk with the mammalian target of rapamycin (mTOR) pathway is induced [8]. TAK1 regulation is less established with regard to AMPK phosphorylation [1] but may be involved in ROS-dependent activation of AMPK [19].

Compared to the AMPK kinases, the protein phosphatases (PP) responsible for removal of the Thr172 phosphorylation are less known. PP1, PP2A and PP2C were all shown to have the ability to dephosphorylate AMPK $[1,20]$. Recently, $\alpha$-SNAP (soluble $\mathrm{N}$-ethylmalemide sensitive factor attachment protein) was added to the list of AMPK phosphatases able to dephosphorylate AMPK [21].

Beside Thr172 phosphorylation, other post-translational modifications can regulate AMPK activity as reviewed elsewhere $[1,8]$. A good example of this regulation is the phosphorylation at Ser485 which is triggered notably by Akt and leads to AMPK inhibition activity [8]. An additional layer of regulation acts through AMPK subcellular localization which can be influenced notably by the CBM, by a nuclear export signal in the $\alpha$-subunit and by different post-translational modifications $[1,8]$.

Altogether, AMPK is a highly regulated kinase responsive to many direct stimuli from 
adenine nucleotide ratios to glycogen and is the downstream target of a series of proteins through post-translational modifications. Not surprisingly, AMPK is involved in a wide number of pathways, which makes the kinase a clear signaling hub.

\section{A signaling hub at the center of metabolic regulation}

In biological signaling networks, most of the proteins interact with few partners, while the minority of proteins forms nodes with tens to hundreds of interactions. The latter are classified as signaling hubs [22]. As a recent interactome study revealed more than 300 protein partners of AMPK in a rat pancreatic cell line [23], AMPK definitely belongs to the category of the signaling hubs (Fig. 2). As such, AMPK is at the convergence of many incoming signals and its activation emerges on an even greater number of responses. In this section, we shortly describe selected inputs and outputs of AMPK signaling.

All the described cellular effectors and proteins act together on AMPK to transmit many different signaling pathways to the kinase, which makes AMPK a convergence point. Most AMPK-activating situations such as exercise, hypoxia and caloric restriction, translate into a change in adenine nucleotide ratios and/or ROS production [14]. Comparatively, the pathway of calcium signaling seems important in only a handful of cases, such as thrombin-induced activation of endothelial cells [24]. Reciprocally, nutrient availability inhibits AMPK through different mechanisms: high ATP levels, direct inhibitory phosphorylation by Akt and activation of PP2A by the lipid intermediate molecule ceramide [1]. Inflammation also inhibits AMPK, possibly via LKB1 downregulation [1]. Despite the extensive amount of known AMPK regulation mechanisms, the mechanisms which underlie some AMPK-modulating pathways still remain to be determined: the hormones adiponectin and leptin for instance activate AMPK in peripheral tissues but the direct AMPK modulating pathway is as yet unknown[2].

As a result of these incoming stimuli, AMPK will act on a variety of pathways according to three main categories: acute adaptation, change in transcriptional activity and modulation of other signaling hubs.

The first category of targets contributes to the acute adaptation of metabolism which quickly stops energy demanding pathways and increases energy production. To take advantage of energy that could be found in the environment, AMPK triggers the translocation of nutrient transporters to the membrane: for GLUT4 translocation, AMPK phosphorylation of TBC1 domain family member 1 (TBC1D1) releases its inhibitory effect on the fusion of vesicles to the membrane [25]. AMPK then diverts entered glucose away from anabolism by inhibition of the glycogen synthase and towards catabolism by activation of glycolytic enzymes such as phosphofructokinases 


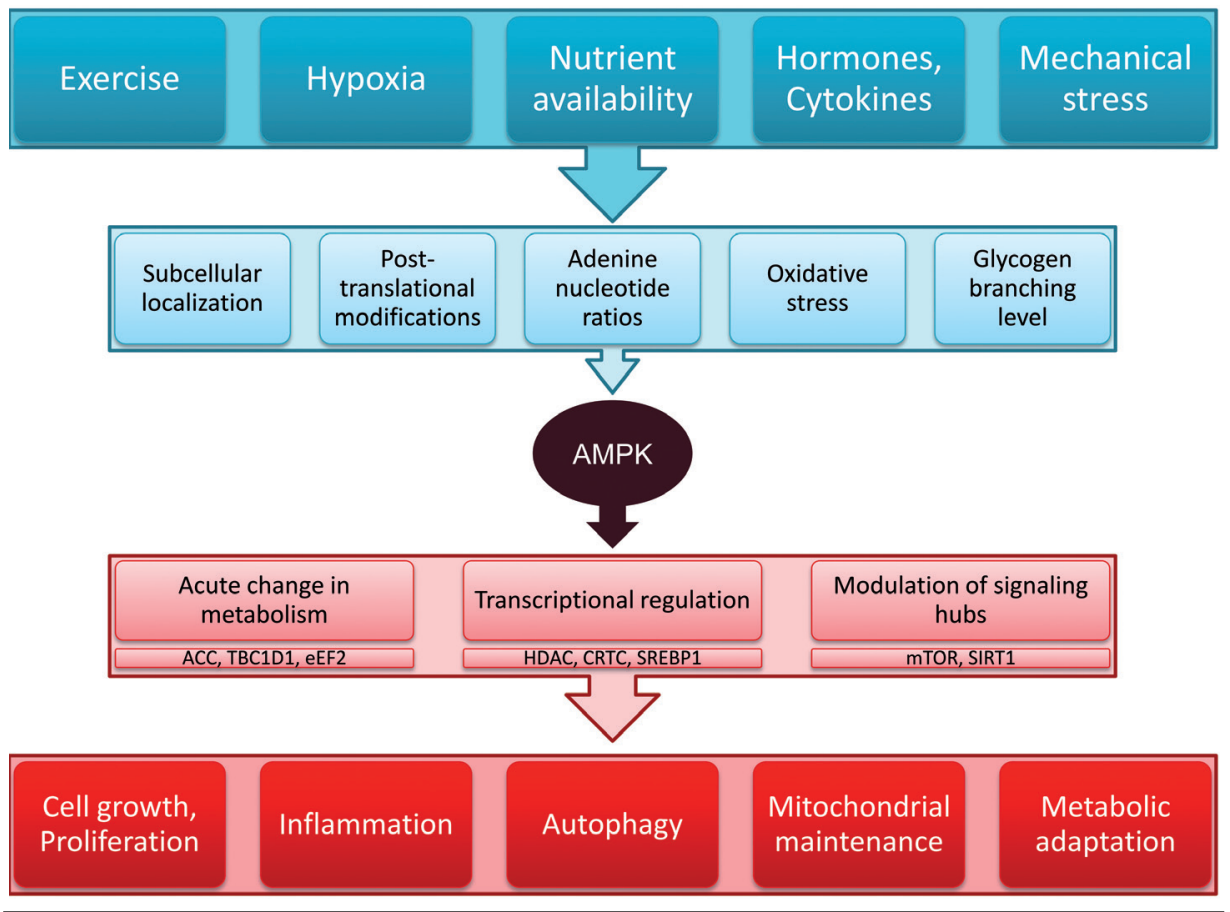

Figure 2. AMPK is a signaling hub where many signals converge (in the darker shade of blue), transmitted to AMPK through a set of direct regulators (in light blue), and from which many pathways start (in light pink) leading to a great number of cellular and whole body effects (in dark pink). Clearly defining which of the direct AMPK regulators are involved in each of the incoming stimuli is complex as these factors are intricate and define together the level of activity of AMPK. Similarly, the many pathways influenced by AMPK lead in fine to the known outputs of AMPK but separating them may be a difficult task as each of them are influenced by the overall status of AMPK. Therefore we propose this image of double-layered inputs and outputs in AMPK biology without clear-cut pathways. Meanings of each abbreviation can be found in the text or in the abbreviation section.

[25]. For adjustment of lipid metabolism, AMPK inhibits the acetyl-coenzyme A (ACC) 2 and thereby lowers the synthesis of malonyl-coenzyme $A$, an inhibitor of mitochondrial import of fatty acid, which results in an increased fatty acid $\beta$-oxidation [25]. Meanwhile, the synthesis of lipids is also halted by phosphorylation of the isoform 1 of ACC. Protein synthesis is also acutely downregulated by AMPKactivity via inhibition of the eukaryotic elongation factor 2 (eEF2) [26] and through other effects further discussed below.

On a more long term basis, AMPK also directly affects gene transcription. For the metabolic adaptation once again, AMPK phosphorylates class II histone deacetylases (HDAC) and CREB-regulated transcription coactivators (CRTC), which triggers their cytoplasmic sequestration by 14-3-3 and thereby prevents the transcription of gluconeogenic enzymes [2,5]. Similarly, AMPK-driven phosphorylation of sterol 
regulatory element-binding protein 1 (SREBP1) results in retention of the transcription factor in the cytoplasm, which prevents lipogenic gene expression [2].

In addition to these acute and long term changes AMPK also influences other signaling hubs which will themselves trigger acute and long term effects. Within this type of targets, mTOR is probably the most established. AMPK phosphorylation activates the inhibitory tuberous sclerosis 2 (TSC2) and prevents the regulatoryassociated protein of mTOR (RAPTOR) from forming the mTOR complex 1 [27]. The resulting $\mathrm{mTOR}$ inhibition leads to downregulation of protein synthesis and cell growth arrest as well as triggers autophagy. For that last effect AMPK also directly phosphorylates unc-51-like kinase 1 (ULK1) which furthermore lacks the inhibition by mTOR in this situation [14]. Through changing the NAD/NADH ratio, AMPK activity also impacts the hub sirtuin 1 (SIRT1) causing mitochondrial biogenesis via the deacetylation of peroxisome proliferator-activated receptor gamma coactivator $1 \alpha$ $(\mathrm{PGC} 1 \alpha)[2]$.

Hence, these few examples demonstrate that AMPK forms a potent signaling hub with many important downstream targets and implication in major metabolic pathways. This role naturally involves it in a wide number of diseases.

\section{AMPK involvement in diseases}

As a center of metabolism, AMPK emerged quickly as a potential target in metabolic diseases and specifically in insulin resistant states. Over the years, many other medical areas discovered a link to AMPK, which brought forward potential therapeutic applications for both activators and inhibitors of AMPK (Fig. 3). We will shortly present these many areas, with emphasis on new domains where AMPK may eventually be considered as a therapeutic target but the stage is still too early now. For more details on more established fields, the reader is referred to the cited reviews.

A primary application for AMPK activation is the treatment of insulin resistance and metabolic diseases [18, 27]. The effects of AMPK on metabolism lead to multiple benefits on key organs: enhancement of fatty acid oxidation in muscle, inhibition of gluconeogenesis in liver, and switch to an anti-inflammatory profile in macrophages are prominent examples of the beneficial effects of the upregulation of AMPK activity in diet-induced insulin resistance $[2,28]$. Therefore AMPK is considered as a prominent target in this area. Similarly, the beneficial involvement in cardiovascular diseases is quite strongly supported $[1,27]$. In the heart, thanks to its ability to notably upregulate autophagy and reverse oxidative stress, AMPK exerts a cardioprotective effect for example during heart failure [14]. In the field of vascular research, upregulation of AMPK activity also appears to have beneficial effects as AMPK is implicated in the nitric oxide (NO) pathway in both endothelial and smooth muscle cells [29]. This pathway is 
of major importance for vasodilation and regulation of immune cell circulation, which together implies a role for AMPK in angiogenesis. In atherosclerosis as well, AMPK activation seems to have beneficial effects through downregulation of endoplasmic reticulum stress and mTOR pathway [29]. Beyond these individual pathologies, AMPK is involved in a number of pathways associated with prolonged healthy lifespan (autophagy, mitochondrial biogenesis, stem cell rejuvenation, etc.) [26]. More than a target in specific diseases, AMPK activation thus arises as a potential method to delay the appearance of all age-related disorders at once.

In contrast to these fairly established links, an association is emerging between AMPK and wound healing. The Murphy Roth Large mice, which carry an homozygous null mutation in the fas genes associated with decreased apoptosis, have a greater ability to heal without steatosis or scars through a yet imprecise mechanism [30]. Mitochondrial dysfunction in these mice was associated with constitutively increased phosphorylation of AMPK [31]. The authors suggest that altered AMPK activation could partly explain the healing phenotype as AMPK is known to downregulate ROS production and decrease fibrosis. Among the other ideas already proposed to explain the increased healing ability [30], several mechanisms overlap with known AMPK actions, for instance enhanced stem cell abilities, alteration of cell proliferation, and variation in metabolic traits and mitochondria. Therefore testing whether the enhanced AMPK phosphorylation causes the healing phenotype could be of great interest.

Peripheral pain plasticity still lacks efficient therapeutics, despite the large number of incidence [32]. The activation of mTOR and mitogen-activated protein kinases (MAPK) such as the extracellular signal-regulated kinase (ERK) were recently implicated in the molecular pathways involved in that field, which suggest that their inhibition could provide therapeutic interest. However, AMPK is advocated as an even better target since AMPK activation was already linked to inhibition of both mTOR and ERK [32]. In line with this, a very new study reports downregulation of AMPK activity in blood samples from chronic pain patients and an amelioration of symptoms with metformin administration [33]. Based on in vitro data using metformin and AMPK inhibitors, the authors propose an AMPK-induced suppression of NOD-like receptor family pyrin domain containing 3 (NLRP3)-inflammasome activation to be causal for the beneficial effect. Even though the latter work requires more direct proofs of action, these results coupled with the mentioned review urge for more data regarding AMPK implication in pain management.

Recently, AMPK was also linked to fertility as AMPK $\alpha 1$ knockout mice have significantly reduced litter size due to abnormal spermatozoa and poor quality oocyte. Similarly, the initiation of germline was linked to AMPK in C. elegans [34]. However, whether AMPK activation could improve fertility in reproductive disorders remains an 
interesting area of research.

Therefore the fields in which AMPK activation may be beneficial count both established and emerging areas, which makes together an impressive number of potential applications for AMPK activators. In comparison, the fields where AMPK inhibition may be beneficial are still rather scarce.

Despite the proven cardioprotective role of AMPK, several glycogen storage disorders as well as the puzzling case of the Wolf-Parkinson-White syndrome link AMPK hyperactivation to cardiac dysfunction [35]. In this latter disorder, a mutation of AMPK $\gamma 2$-subunit renders the kinase both insensitive to adenine nucleotides and highly active, which leads to left ventricular hypertrophy and cardiac pre-excitation [14]. Patients who carry such mutation would therefore probably benefit from a cardiacspecific AMPK inhibitor. Similarly to these paradoxical data in heart which show AMPK activity as both beneficial and detrimental, a recent report shows that AMPK activation induces insulin resistance in the specific case of nicotine consumption [36]. Whether patients with nicotine-induced insulin resistance could benefit from AMPK inhibition deserves more investigation as use of these inhibitors may also lead to a metabolic imbalance.

In the case of cancer, application of AMPK modulators may be of interest but their use will probably have to be individually set [5,37]. Indeed, as an inhibitor of mTOR and thereby of cell growth and proliferation, AMPK represents a tumor suppressor downstream of LKB1. But, as a metabolic regulator activated by stresses, AMPK can also enable tumor survival by offering adaptation to the hostile environment. Similarly, the implication of AMPK in neurodegenerative diseases cannot be judged as universally beneficial or detrimental. Whereas AMPK activation prevents neuronal death in Parkinson's disease, it increases it in a model of Huntington's disease [26]. In Alzheimer's disease, evidence for both a beneficial activation and inhibition of AMPK have been gathered [6]. Finally in stroke, the lack of oxygen and nutrient associated with the blood shortage activates AMPK. However, activation of AMPK may not always be beneficial, as many contradictory results have been found on the effects of AMPK modulators and knockouts [1]. The duration and intensity of the hypoxia-induced AMPK activation seems to be the key to predict the outcome [38]. Finally, a fairly new domain in which AMPK inhibition may be required is that of intracellular pathogens [39]. Similarly to cancer though, the AMPK/mTOR pathway may both provide the pathogens with energy necessary to their survival, but may also be a required system to fight pathogens through autophagy.

In summary, as a central signaling player in many pathways, AMPK is emerging as a potential therapeutic target in a wide number of disorders. Both AMPK activation and inhibition may be desired as therapeutic treatment in different situations. Several 


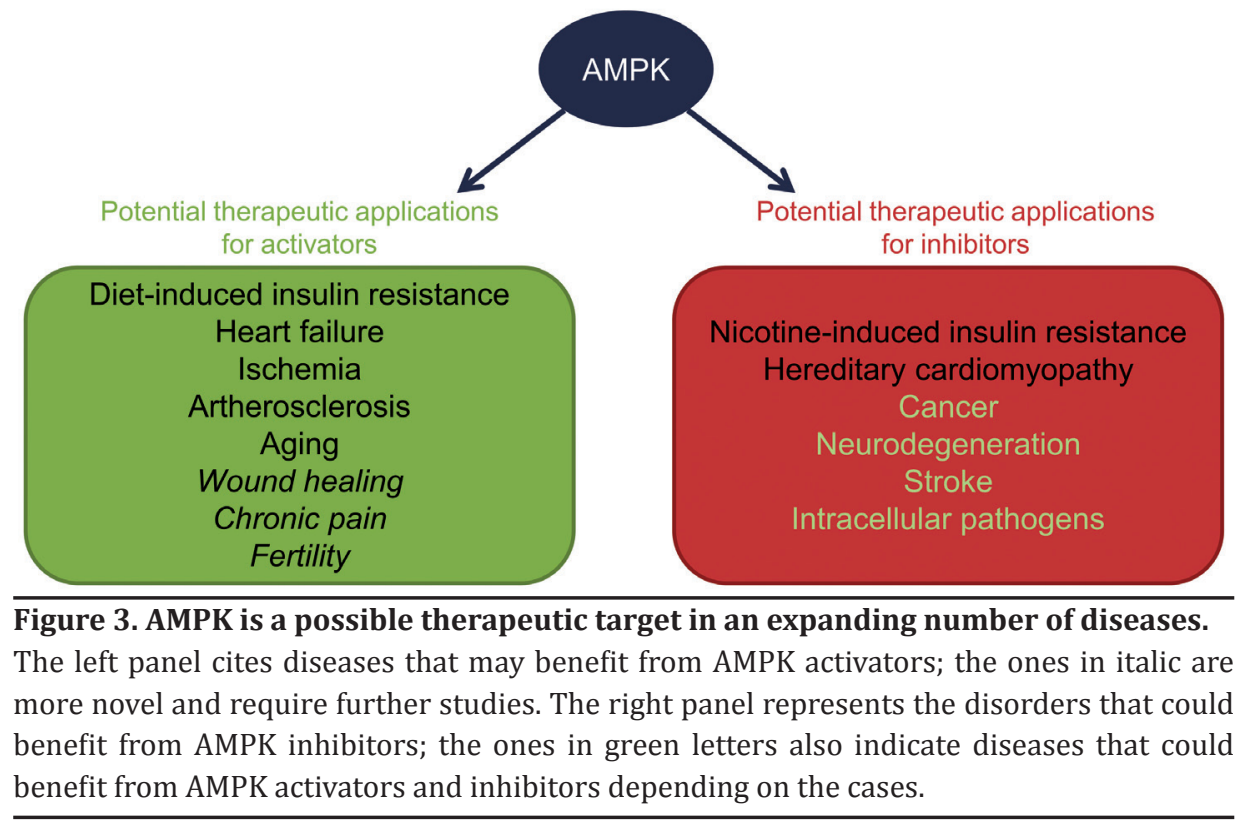

direct AMPK activators and inhibitors have been already described but their application does not reach the expectations of such a potentially relevant therapeutic target, leaving opportunities for innovation.

\section{Increasing evidences of the need for more specific modulators}

Although activation and inhibition of AMPK could be relevant for therapeutic intervention, both targeting approaches could lead to unwanted side-effects. Therefore a more selective strategy should be undertaken. Interestingly, AMPK is composed of three subunits existing in different isoforms, each of which is known to serve different in vivo functions and with differing intrinsic specificities that could possibly be taken advantage of. The knowledge on isoform specificity seems indeed to indicate an exploitable strategy in some cases. However, the currently available modulators do not meet the requirements of specificity.

\section{The potential secondary effects of AMPK targeting}

Since AMPK activation and inhibition are implicated in different states of life or diseases, therapeutic approaches which use either AMPK activators or inhibitors may imply the risk to set in motion the pathological aspect of the other side of the modulation: for example, by activation of AMPK to facilitate a potential tumor progression and by inhibition of AMPK to set the metabolic balance off. Beyond this fear, which in the end will have to be the object of a risk versus benefit study for each targeted disease, 
potential secondary effects of AMPK targeting are known.

In the case of AMPK activation, the effect on the brain may be the most worrisome. As a kinase primarily activated upon energetic stress, a logic downstream effect is the upregulation of food intake to compensate the need for energy. Accordingly, hypothalamic AMPK is activated by hypoglycemia and upregulates food intake [1]. In metabolic syndrome, upregulation of appetite as secondary effect of AMPK activation would obviously be worsening the overnutrition that is seen as a major cause of the disease. However, in support of the beneficial activation of AMPK, the many indirect AMPK activators used in the clinic (metformin and thiazolidinediones) argue that activation of AMPK is a safe strategy, but of course, the activation of AMPK alone may still lead to a different outcome than that of the multi-targeted approach.

Regarding AMPK inhibition, knock-out mice can give us an idea of the long term inhibition consequences. While $\alpha 1$ whole body knock-out had a normal energetic homeostasis, the $\alpha 2$ null mice showed a mild insulin resistance and glucose intolerance [40]. Interestingly, this impaired glucose metabolism was linked to a dysregulation of the autonomic nervous system and hypothalamus, which points once again to the delicate case of cerebral AMPK targeting. While AMPK knock-out mice that completely lack AMPK activity are not viable, tissue specific deletions of AMPK have been achieved [40], but how these models would relate to a pharmacological targeting in the whole body is difficult to assess. An interesting case of pharmacological inhibition is that of sunitinib, a multi-targeted tyrosine kinase inhibitor used in the clinic for its antiangiogenic and antitumor activity. Being an ATP-competitive molecule with promiscuous effects on many kinases, sunitinib targets AMPK with an even better affinity than that of the more established AMPK inhibitor, Compound C $[41,42]$. While the multi-targeting effects of sunitinib may explain its efficiency on cancer cells, it also causes cardiotoxicity. A first study dismissed AMPK inhibition as explanatory of this side effect: although AMPK was indeed inhibited by sunitinib, ATP levels were not affected and metformin pre-treatment was unable to rescue myocytes from sunitinibinduced toxicity [43]. However, only cardiac toxicity was monitored during this latter experiment, and whether AMPK could truly be activated by metformin under these conditions was not proven. In contrast, another team described sunitinib-induced mitochondrial dysfunction in both patient cardiac samples and in isolated neonatal rat ventricular myocytes, which was associated with reduced ATP levels [42]. Although AMPK phosphorylation was high, the phosphorylation of its downstream target was low, which testifies to a direct inhibitory effect of sunitinib on AMPK catalytic activity. In contrast, over-expression of a constitutively active AMPK blunted the sunitinibinduced myocyte toxicity. Hence, AMPK inhibition may not be the only cause of sunitinib-induced cardiotoxicity but sunitinib surely relieves the AMPK cardioprotective 
effect. Similar results were found with another chemotherapeutic agent, doxorubicin, for which the cardiotoxicity may also be linked to AMPK inhibition [14]. Even though these results point to a potential detrimental effect of long-term AMPK inhibition, the benefits in diseases such as cancer may still exceed the increased risk of toxicity. In other disorders such as stroke, acute inhibition may give satisfactory results without leading to cardiotoxicity.

In summary, as with many other targets, AMPK activation and inhibition may both lead to unwanted side-effects and the pharmacological modulation of AMPK will need to be carried out with precaution. An exploitable strategy to target AMPK in a more specific manner would be to take advantage of the multiplicity of AMPK trimeric composition and target isoforms specifically.

\section{A multiplicity of AMPK targets with different roles}

As mentioned above, AMPK is not a unique molecule but exists in many different isoforms which together may form up to 12 different hypothetical complexes [1]. The composition of AMPK varies in time, with age and between health and disease, and in space, between tissues and within the cell. However, AMPK is often considered as a single molecule in physiological studies and functional investigations are routinely restricted to the main complex $\alpha 1 \beta 1 \gamma 1$ [44], disregarding potential isoform assignment of specific pathways. Still some elements of isoform-specific roles and regulation have been reported and these will be summarized below.

AMPK tissue specific expression profiles in humans have been reported in several studies. The muscle is the organ with the most specific expression set: the $\alpha 2$ and $\beta 2$ are more prevalent in these tissues than others, while $\gamma 3$ is exclusively expressed in the skeletal muscle [35]. Actually, this latter tissue expresses predominantly $\alpha 2 \beta 2 \gamma 1$, followed by $\alpha 2 \beta 2 \gamma 3$ and $\alpha 1 \beta 2 \gamma 1$, but none of the other possible complexes $[1,35]$. The picture in other organs is less clear, but the subunits $\alpha 1, \beta 1, \gamma 1$ are quite ubiquitously expressed throughout the body [44]. Interspecies differences in tissue expression have also been reported indicating that AMPK may be differently regulated among organisms and that conclusions from animal models should not be directly extended to humans without precaution. In liver notably, $\beta 2$ is the main subunit in humans while mice hepatocytes express predominantly $\beta 1$ [45].

Isoform-specific regulations have also been reported. In the rat pancreatic cell line INS1, AMPK $\alpha 2$-containing complexes were found more predominant in the nucleus compared to $\alpha 1$ [46], which suggests that the transcriptional effect of AMPK may be principally carried out by $\alpha 2$. In the same study and in another, AMPK complexes containing the $\alpha 2$-subunit was found more sensitive to AMP effects than AMPK that contains $\alpha 1$ [46, 47], implying that pathways that elevate the levels of AMP may 
primarily act through the $\alpha 2$ subunit. For the $\beta$-subunits, the CBM of $\beta 2$ binds oligosaccharides with a 5-fold better affinity - even 15-fold for branched oligosaccharides - compared to that of $\beta 1$ [48]. Although it was not formally shown in cells, this difference suggests that $\beta 2$-containing complexes are more likely to be found at glycogen in cells than those with $\beta 1$, which could suggest a stronger link of $\beta 2$ containing trimers in glucose regulation. Finally, $\gamma$-subunits respond differently to adenine nucleotide, with $\gamma 2$ having the greatest sensibility to AMP, followed by $\gamma 1$ and finally $\gamma 3$ [47]. Additionally, in cardiomyocytes and skeletal muscle fibers, immunofluorescence studies revealed different localizations for all three $\gamma$-subunits, which points towards different roles [49].

Specific roles may also be separated between isoforms by study of knock-out models, although this is harder to judge due to possible compensating effects $[40,50]$. Still, the $\alpha 2$ knock-out mice present a dysregulated metabolism while mice with deletion of $\alpha 1$ keep a normal insulin response [40], implying a stronger involvement of $\alpha 2$ complexes in metabolic regulation. In pancreatic $\beta$-cells, glucose-induced insulin secretion requires the inhibition of $\mathrm{AMPK}$ and although the $\beta 1$-subunit is predominantly expressed, knock-down of $\beta 2$ is sufficient for derepression of insulin release [51].

Thus there are ample indications that AMPK complexes are different across tissues and subcellular localizations, and play separate roles, which points to diversified, almost unique, molecules. It is interesting to know whether AMPK complex composition changes in health and disease, which could help to define strategies to reestablish the healthy expression profile. Such a case was found in the failing heart, where $\alpha 1, \beta 1$ and a specific splice variant of $\gamma 2$ are upregulated [52]. In human primary breast cancer cells, the expression of AMPK $\alpha 2$ was downregulated compared to healthy neighboring tissue while $\alpha 1$ levels were maintained [53]. Interestingly, the overexpression of this subunit in MCF-7 cells promoted apoptosis under energetic stress whereas its silencing suppressed the growth inhibition upon low glucose conditions, which suggests a tumor-suppressive role of AMPK $\alpha 2$ in this type of cancer. In the case of diabetic liver however, no change in AMPK composition was observed compared to healthy controls [45].

In summary, AMPK complex constitution clearly impacts on its regulation and role, and is thus an important aspect to take into account for development of targeting strategies. Although key residues are fairly highly conserved between isoforms, inserted/deleted regions together with single amino acids differences are still scattered throughout the sequences offering possibilities for specific targeting (Fig. 4A). In particular, the $\gamma 2$ and $\gamma 3$ subunits have extended $N$-terminal sequences absent from $\gamma 1$, although a role for these sequences have not yet been determined [47]. Several examples of AMPK direct activators exist already: the strategies for their development 

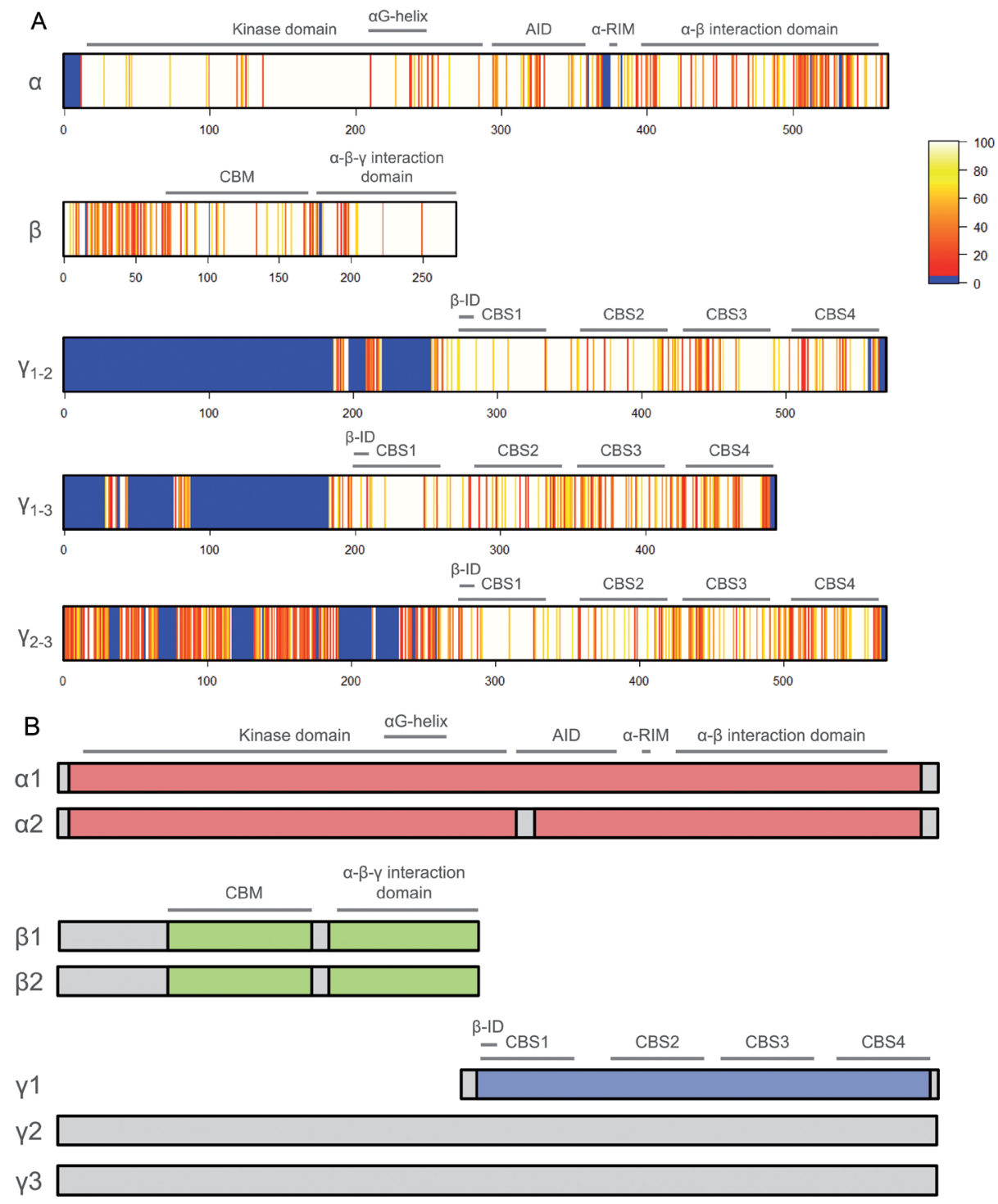

\section{Figure 4. AMPK isoform sequence conservation and availability of structural informa- tion}

(A) Conservation of amino acid sequences between AMPK isoforms. Scores of conservation between two related isoforms were retrieved from alignments using ClustalX2 and plotted using the R software (score of 0-5: deletion/insertion represented in blue, 5-99: degree of conservation in a gradient from red to yellow, 100: same amino acid in white, as depicted in the legend on the right). X-axis numbering represents residue number but is a combination of the two compared isoforms. Based on these alignments, the percentage of sequence identity is $74 \%$ for $\alpha 1 / \alpha 2,70 \%$ for $\beta 1 / \beta 2,42 \%$ for $\gamma 1 / \gamma 2,41 \%$ for $\gamma 1 / \gamma 3$, and $41 \%$ for $\gamma 2$ / $\gamma 3$

(B) One-dimensional representation of AMPK subunit isoforms indicating the availability of 
high-resolution structural information. Wide regions of AMPK are structurally resolved, but specific isoform structures especially of the $\gamma$-subunit are left to determine. In grey are the unknown parts while colors show the regions with available structure(s) for mammalian constructs (see Supp. Table 1).

Key domains are highlighted with grey bars. AID: auto-inhibitory domain; $\alpha$-RIM: $\alpha$ regulatory subunit interacting motif; CBM: carbohydrate-binding module; $\beta$-ID: $\beta \gamma$ interaction domain; CBS: cystathionine $\beta$-synthase.

and their current shortcomings are presented in the next section.

\section{Known direct AMPK activators}

Although many compounds have already been described to activate AMPK, those with a known mode of action can be classified in either of three categories. Here we present only the main compounds of each family (Fig. 1, Table 1); more extensive reviews of all existing derivations can be found elsewhere [6, 54, 55]. Similarly, many indirect activators of AMPK, which generally influence the AMP/ATP ratios, have been established, among which marketed diabetes drugs - biguanides (metformin) and thiazolidinediones for instance - and natural compounds such as resveratrol or berberine. In this section we focus on direct activators only, and once again refer the reader to more extensive AMPK modulator reviews for details $[6,54]$.

The first mode of action for direct activators is to mimic a change in adenine nucleotide ratios. The 5-aminoimidazole-4-carboxamide ribonucleotide (AICAR) is a prodrug which is transformed after entry in the cells into ZMP, an analog of AMP [56]. The rise in ZMP which results from AICAR treatment activates AMPK in cells and in vivo [27]. This action is mediated through ZMP direct binding to the adenine sensing sites of the $\gamma$-subunit of AMPK and therefore ZMP triggers all three activating aspects of AMP (allosteric activation, protection against Thr172 dephosphorylation, trigger of phosphorylation) $[27,57]$. However, as a mimic of AMP, AICAR presents many offtarget effects on other AMP sensitive enzymes, such as fructose-1,6-bisphosphatase-1 [27]. The cellular stress mimicked by AICAR also triggers in an AMPK-independent manner the synthesis of bis-diphosphoinositol tetrakisphosphate $\left([\mathrm{PP}]_{2}-\mathrm{InsP}_{4}\right)$ from which pyrophosphate groups are directly used to phosphorylate proteins independently of kinases [58]. Therefore the use of AICAR to identify potential downstream targets of AMPK may not be suitable as AMPK- and $[\mathrm{PP}]_{2}$-InsP $\mathrm{I}_{4}$-induced phosphorylations may overlap. Several structure-activity relationship studies were carried out to determine important features for potency and selectivity of AMP analogs towards AMPK [6]. Among the molecules that were discovered through such studies, the Compound 2 (Compound 13 as prodrug) issued from the screening of AMP analogs, is a very potent activator of AMPK in cells and rodents while it shows an improved specificity towards 


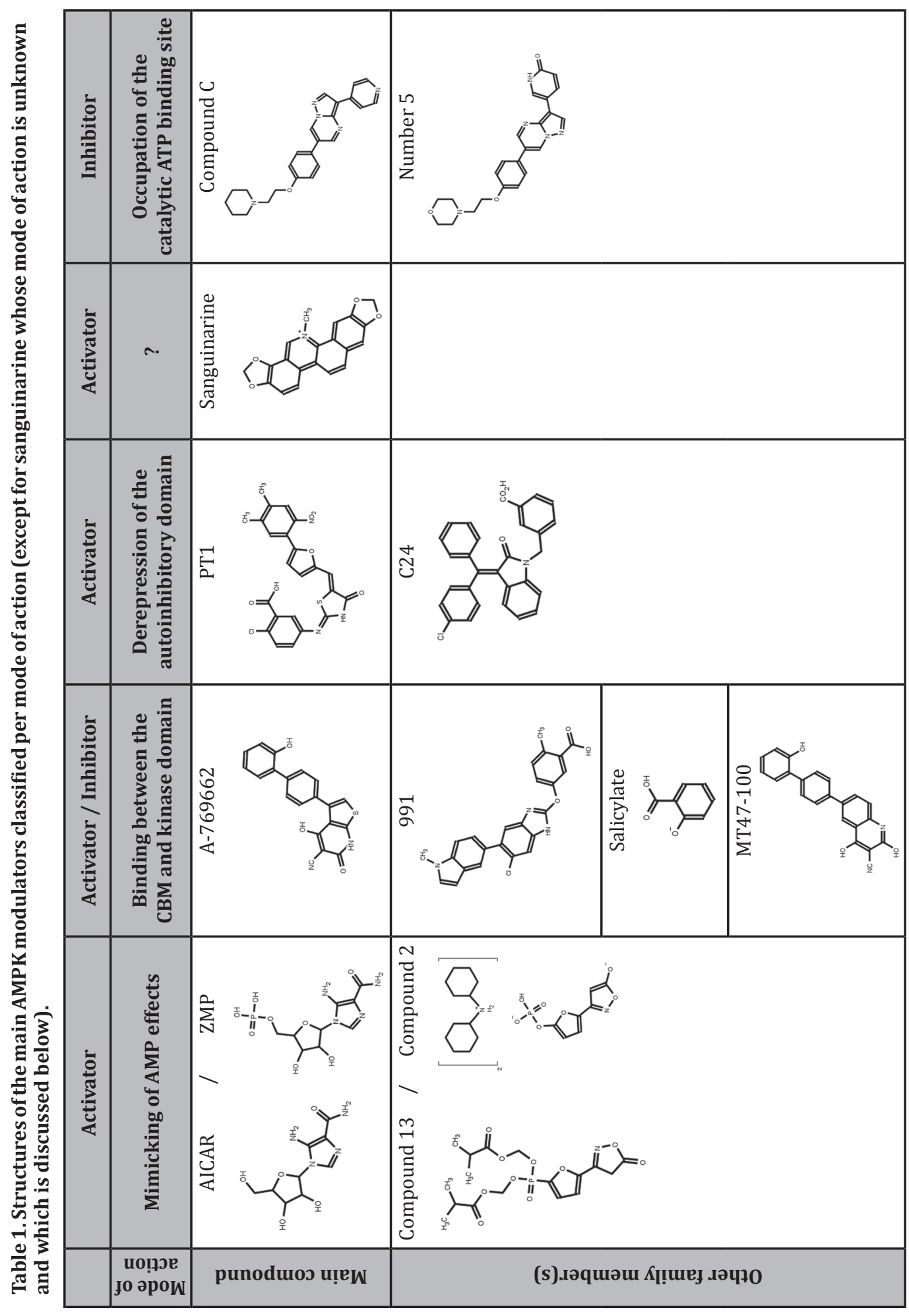


AMPK compared to fructose-1,6-bisphosphatase-1 for instance $[59,60]$.

In the family of the second mode of action, the hit that led through optimization to A-769662 was identified in a screening conducted by the Abbott Laboratories using kinase activity of purified rat liver AMPK as readout [61]. Although A-769662 activation showed all three components of AMP-induced activation, the modes of action of the two molecules are separate. The co-crystallization of an AMPK trimer and A-769662 revealed that the activating compound stabilizes the interaction of the $\beta$-subunit CBM on the $\alpha$-subunit kinase domain, forming a pocket for which the phosphorylation of $\beta$-subunit Ser108 is required [7]. Despite a good activity in vivo, the compound presents poor bioavailability as well as some off-target effects [27]. Nevertheless, other work led to the identification of more activating compounds with a different scaffold, similar properties and improved absorption [6]. In the direct line of A-769662, the compound 991 was reported to possess 5 to 10 -fold increased potency [7]. Interestingly, salicylate, a plant product from which Aspirin is derived, acts on AMPK presumably in the same mode, although very high concentrations are needed to see an effect [62].

The family of the third mode of action started with the discovery of PT1, which was identified in a screening with the $\alpha$-subunit alone, in which case kinase activity is repressed by the auto-inhibitory domain under basal conditions [63]. PT1 activated the isolated $\alpha$-subunit and the trimeric complex but not the isolated kinase domain without autoinhibitory domain, which suggests that PT1 directly binds to the AMPK $\alpha$-subunit and derepresses autoinhibition. Unfortunately, PT1 showed very poor bioavailability and was inactive in rodents [64]. A recent study of PT1 derivatives [65] disclosed the identification of $\mathrm{C} 24$ with similar activity properties to isolated $\alpha$-subunits but improved potency, bioavailability and activity in vivo [64]. However, a study from a different group casts doubt on the mechanism of action of PT1 and related compounds since, in their hands, PT1 failed to activate AMPK $\alpha$-subunit in vitro, elevated the AMP/ ATP ratios in treated cells and a good part of PT1-induced cellular AMPK activation was prevented by overexpression of an AMP-insensitive AMPK mutant [66]. Therefore PT1 may to the least have also indirect effects on AMPK activation and a detailed description of its mechanism of action requires further experimentation.

All these direct activators and especially AICAR and A-769662 are extensively used for scientific purposes $[1,6,27]$ but due to their limited bioavailability and off-target effects, they failed to reach the clinics. However, with AMPK being such a relevant drug target, many efforts are still pursued to find the right targeting strategy. Beyond these problems of bioavailability and specificity, the currently available activators also have a long way to go with respect to isoform-specific targeting. 


\section{Are current AMPK activators specific?}

Considering the increasing knowledge on the separate involvement of different AMPK complexes and the known risks of systemic AMPK modulation, isoform-specific targeting has been proposed as a possible safer approach for therapeutic AMPK activity modulation. However the currently known AMPK activators do not really meet the expectations in that field.

The main AMPK activator considered as reasonably specific is A-769662. This compound was derived from the hits of a screening based on partly purified complexes from rat liver so mainly containing $\alpha 1 \beta 1 \gamma 1$ [61]. After expression in mammalian cells and purification, the 12 different AMPK complexes were tested for activity and only $\beta 1$-containing trimers were sensitive to A-769662 [67]. Consistent with this, A-769662 failed to activate AMPK in muscle of $\beta 1$-null mice $[62,67]$ and did not trigger glucose uptake under normal concentrations in WT muscles which express mainly $\beta 2$ over $\beta 1$ $[67,68]$. However, $\beta 2$-containg complexes can bind A-769662 with a low micromolar dissociation constant $(\sim 14.5 \mu \mathrm{M}$ for $\alpha 1 \beta 2 \gamma 1)$, much higher than that of $\alpha 1 \beta 1 \gamma 1$ $(\sim 0.51 \mu \mathrm{M})$ [7], but still reasonable for a compound used in scientific investigations. Although A-769662 failed to trigger a significant allosteric activation of the $\beta 2$ complex, a similar compound 991, which is a much better binder, could activate AMPK independently of the expressed $\beta$-subunit [7]. Since A-769662 was only tested at 10 $\mu \mathrm{M}$, the data with 991 suggest that A-769662 may also act on allosteric activation at higher concentrations. Besides, the protection of dephosphorylation conferred to $\beta 1$ containg complexes by A-769662 was also reported with $\beta 2$-containing trimers [62]. Therefore the assertion that A-769662 is a $\beta 1$-specific activator should be made with precaution, and a $\sim 30$-fold isoform preference (of $\beta 1$ : $\beta 2$ ) may be more accurate to describe the phenomenon. Besides, even if A-769662 or another molecule could be clearly identified as a $\beta 1$-specific compound, the opportunity of such specificity in human metabolic disease is uncertain. The positive effect of A-769662 in the genetic diabetic model of ob/ob mice was shown through its impact on the liver [61]. Since humans express principally $\beta 2$ in liver as well as in muscle [35, 45], the result of AMPK activation by A-769662 will be restricted to other tissues, which may not be sufficient to exert the beneficial metabolic effect.

An isoform preference is also seen for AICAR and AMP action on AMPK trimers: as mentioned above, AMPK containing the $\alpha 2$ are more sensitive to both components [ 69 , 70]. Although we did not find trace of such study, one could hypothesize that this preference also applies for indirect AMPK activators since they act through AMP binding. Interestingly, the prodrug $\mathrm{C} 2$, which is derived from a library of AMP analogs and becomes the active molecule $\mathrm{C} 13$, acts preferentially on $\alpha 1$-containing trimers and 
not $\alpha 2$ as AMP does [60]. A chimeric construct of $\alpha 2$ containing the $C$-terminal region of $\alpha 1$ (which overlaps with the $\alpha$-RIM sequence) rescued the lack of affinity. Although the effect on AMP binding was not assessed, this experiment seems to pinpoint the residues responsible for isoform preference of AMP and its analogs to be directly present within the areas that regulate adenine nucleotide binding.

In more recent targeting studies, the natural compound sanguinarine was identified through a FRET-based screening of recombinant AMPK trimers [71]. Its activity was shown to be restricted to complexes that contain $\alpha 1$ and $\gamma 1$ but not $\alpha 2$ and $\gamma 2$, independently of the $\beta$-subunit present, which suggests a separate mechanism from both AMP and A-769662. However, the exact mode of action for this compound is largely unknown at this stage. Even though the virtual docking of the compound was performed on a homology model of trimeric AMPK that was based on the known core structure of yeast ortholog Snf1, the results are doubtful taken into account that the mammalian trimeric AMPK structure solved since then [7] shows quite some differences with the yeast structure, probably due to the omission of the kinase domain in the latter.

Therefore, although AMPK isoform specific targeting is a declared goal of the AMPK community, which is supported by the data on isoform differences, the available activator molecules fall short of expectations. However, for AMPK inhibitors, this area is even less advanced.

\section{The near-absence of specific AMPK inhibitor}

The first identified AMPK inhibitor, Compound C, was found by high throughput screening based on in vitro activity of AMPK purified from rat liver [72]. The initial study tested the selectivity for AMPK compared to related kinases and presented the compound as seemingly specific. However, Compound $\mathrm{C}$ is now known to be a very promiscuous inhibitor, which targets notably the adenosine transport system thereby preventing AICAR import, which prevents the ROS production and which triggers preadipocyte proliferation independently from AMPK [1]. Because it targets the ATPbinding site of the kinase catalytic site [72], which forms a structurally highly conserved pocket (Fig. 4A), Compound C probably has a similar effect on both AMPK isoforms, but beyond that, it also targets a great number of kinases with similar potencies [74].

To overcome this lack of specificity and improve its potency, a rational design approach was taken in which a model of AMPK $\alpha 2$ kinase domain was built and used to dock Compound C [74]. A library of fragments was then screened for its propensity to fit into the identified pocket and the results were used to design hypothetical binders. Following a structure-activity relationship study, a new compound (number 5) was identified with better potency in cells and with an improved selectivity profile although 
it was still potent on many other kinases besides AMPK.

Surprisingly, a new $\beta 2$-specific AMPK inhibitor was derived from A-769662: the compound MT47-100 retains activation capacity for $\beta 1$-containing trimers, but it inhibits $\beta 2$ complexes[51]. Such profound isoform specificity had never been described before and is of great interest. Even though the compound requires the CBM for binding to both isoforms, the phosphorylation at $\beta$-subunit Ser108 is only necessary for the activation process, suggesting that the binding mode may still differ between isoforms. The inhibition property was applied to derepress the glucose-induced insulin release in pancreatic $\beta$-cells, which is known to be a role preferentially occupied by $\beta 2$ containing AMPK. Although this beneficial outcome gives a first application for this compound in metabolic disorder, treatment on the whole body scale may not be so beneficial for the metabolic disorders in this disease context since liver and muscles would be the main targets of inhibition and are capital organs to store and use nutrient excess. An interesting application could however be the hyperactive mutant AMPK found in Wolff-Parkinson-White syndrome since the detrimental effect is localized to the $\beta 2$-expressing heart tissue.

Despite the recent discovery of MT47-100, which is only partly an AMPK inhibitor, the design of AMPK inhibitors still has a long way to go before reaching expectations. Considering that isoform-specific targeting still seems a valuable approach, both activator and inhibitor design could benefit from novel strategies to target the kinase.

\section{Recent insights into AMPK structure for novel targeting strategies}

As mentioned above, AMPK is in itself a complex structure of three subunits with interregulation between domains. The recent resolution of quasi-complete AMPK trimers brought important advances in understanding how AMPK is regulated notably with regard to adenine nucleotide sensing $[7,17,75]$. These new data add an important chapter to the collection of experimentally resolved AMPK structures (Supp. Table 1). The number of described AMPK modulators is also continuously growing [6]. A logic development in AMPK drug design is therefore the use of computer-aided techniques which would take advantage of the wide amount of available knowledge and allow for novel strategies to be undertaken, notably that of isoform-specificity.

\section{The valuable tools of computer-aided drug design}

Virtual screening comprises a computer-aided set of techniques that help to prioritize which compounds are most likely to bind with high affinity to a target protein and which are most suitable for subsequent in vitro testing [76]. Although the prediction of active compounds obviously does not replace their actual testing in vivo, virtual screenings can spare a lot of time and money by limitation of the amount of biological 
tests that need to be carried out [77]. Computer-based screenings use databases which generally contain a number of compounds in the same range as the actual libraries screened in vitro by the biggest pharmaceutical companies (typically several hundreds of thousands to a million) [76]. A virtual screening effort therefore replaces the need for the physical on site establishment of such libraries that are very expensive to maintain. Moreover, the whole chemical space is estimated to $10^{33} \mathrm{drug}$-like molecules [78] and is therefore much wider than the biggest available library. Hence selection of compounds from various sources allows for the testing of different sets of compounds than those locally available [76]. Different compound databases exist for computeraided screenings based on the type of the target chosen: for instance targeted libraries for kinases and for receptors have been designed [76].

To define the criteria needed for this prioritization, two different, although not exclusive, methods of virtual screening have been defined [77]. The first one is the structure-based virtual ligand screening which requires the 3D structure of the studied protein determined by X-ray or NMR data or predicted by homology modeling. These structures are used to define potential regions, such as pockets or interfaces, which will affect the protein regulation, notably by disruption of the interaction between binding partners or by allosteric modulation [77]. Ideally, in silico predictions will be supported by mutation data. The database of compounds is then assessed by virtual docking onto those regions and subsequent scoring of docked poses to define which ligands are the most likely binders. The second method of virtual screening is a ligand-based technique, which does not require any prior knowledge of the target structure. The comparison of known binders and non-binders contributes to a description of key features in threedimension space (pharmacophore) or in two dimensions (2D fingerprint), or to the prediction of key properties (quantitative structure activity relationships) [77]. The ligands of the virtual database are then scored according to their fit to these criteria. Successful drug design projects using these virtual techniques generally combine both ligand- and structure-based methods for an optimal outcome [79].

Apart from small molecule work, virtual techniques also offer predictions of protein interaction with peptides [80]. Therefore, the design of peptides for pharmacological targeting can also be helped by computer-aided techniques. Besides, this technique also predicts sites for interaction between protein partners and can therefore pinpoint the region to be targeted by small molecules.

Today, several marketed drugs originate from the use of such computer-aided techniques and their usefulness needs no longer to be proven [79].

\section{Opportunities for computer-based drug design in the AMPK field}

In the AMPK field, the activators A-769662, PT1 and the main inhibitor Compound C 
were derived from classical in vitro screens of compound libraries $[61,63,72]$, while AICAR was identified in a search for AMP mimetic compounds [81]. A few optimization studies reported the use of computer-based techniques [64, 74]. Hence, taken into account the amount of data of known binders (Table 1) and AMPK structure (Supp. Table 1), it is safe to state that the AMPK targeting field still has many opportunities for exploration by computer-aided drug design techniques.

An interesting field which has only been brushed by the AMPK targeting community is that of protein-protein interaction (PPI) modulation. Protein interaction interfaces are generally shallow, hydrophobic and wide, which makes them very different from conventional pockets targeted in enzymes and receptors [83]. To target these large pockets with small drug-like compounds, key residues, called hotspots, that contribute to most of the binding free energy need to be identified $[83,84]$. Despite its relative novelty, the field of PPI modulation and particularly inhibition already counts several success stories $[83,84]$. In the case of AMPK, the trimeric nature of the kinase makes it an interesting target for PPI modulation. The stability of each subunit depends to a great extent on the presence of the two others as complete knock-out of one of the three subunits (in all isoforms) generally leads to the disappearance of the whole complex (example in [50]). In addition to degradation, the activity of the kinase also depends on the trimeric form since the $\alpha$-subunitalone is repressed by the autoinhibitory domain [63]. Therefore, a possible inhibitor of AMPK could be derived by targeting the subunit interface, and more specifically the $\beta$-subunit domain tethering $\alpha$ and $\gamma$ together, so as to disrupt the formation of a trimeric state. To design such inhibitor the resolved structure of the core of AMPK would be helpful to define key residues to be targeted $[7,12]$. Apart from full trimer disruption, key domains could also be targeted by PPI inhibitors. The interaction between the CBM and the kinase domain enhances AMPK activity [17] and allows for the activators of the A-769662 compound class to bind. Similarly, the contact between the $\alpha$-RIM and $\gamma$-subunit transmits the adenine nucleotide status to the kinase domain [12] and can therefore be activating. In these two examples, interaction stabilizers have already been described, i.e. A-769662 (or 991) and AICAR/ZMP (or C2/C24) respectively. The former compounds were cocrystallized in one of the latest resolved structure [7] and for the latter, the active substance ZMP was co-crystallized in Snf1 [85] and could be easily modeled after its mimetic molecule AMP in the mammalian complex [12]. The poses of the agonist compounds could then form the structural base to identify bulkier antagonist compounds which would still bind in the same places but disrupt the activating interactions, thus resulting in inhibition. Although the binding mode of MT47-100 is unknown at this stage, the activation or inhibition seen in $\beta 1$ and $\beta 2$-containing trimers respectively could be due to a difference in pocket topography between the two 
isoforms, which would make the compound to behave either an agonist or an antagonist respectively.

Furthermore, the central signaling role of AMPK requires its interaction with a great amount of partners, for which only a few binding sites have been identified thus far. Beyond AMPK trimeric structure, the kinase is known to associate with other AMPK complexes to form di- or even multimers [86]. This, at least in part, involves hydrophobic residues of the $\alpha \mathrm{G}$-helix of the kinase domain [86]. When this helix is mutated, AMPK oligomerization efficiency goes down but so does the AMPK activity, as the mutations also limited interaction with both LKB1 and PP2C $\alpha$. The $\alpha \mathrm{G}$-helix thus appears as a critical binding site for AMPK interactions. Interestingly, the strategy of targeting this binding site has already been followed with the computational design of peptides resembling the $\alpha$ G-helix of AMPK [87]. Surprisingly, instead of AMPK inactivation as might be expected from the mutant data, treatment with the peptides activated AMPK in myocyte and ameliorated metabolic parameters in high-fat fed mice. Whether the latter effect was really due to AMPK targeting was not studied, neither was the reason for AMPK activation. Nevertheless this first study offers an interesting starting point for further targeting. Another binding site which could be relevant for AMPK activation is the $\beta$-subunit. The ubiquitination of AMPK depends on the formation of a complex that contains the cell death-inducing DNA fragmentation factor $\alpha$-like effector A (Cidea) [1]. The interaction of Cidea and AMPK which leads to AMPK ubiquitination and degradation was located within a 12-amino acid region on AMPK $\beta$-subunit, near the $\alpha \gamma$-tethering domain. This region is present in the core AMPK structure [12] and could therefore be the base of a targeting strategy to prevent AMPK degradation.

Apart from protein interactions, AMPK is also known to bind carbohydrates, and particularly glycogen. This interaction is carried out by the CBM of the $\beta$-subunit which has been crystallized for $\beta 1$ as a holo form in complex with $\beta$-cyclodextrin [88] and for $\beta 2$ both as an apo form (unpublished, PDB ID: 2F15) and a holo form with a glucosyl- $\beta$ cyclodextrin [89]. NMR data are also available for $\beta 2$ in both apo and holo forms (unpublished, PDB ID: 2LU3 and 2LU4). This amount of structural data, coupled with further extensive studies on oligosaccharide binding [48], gives a good start for the design of small molecules to prevent the binding of carbohydrate to AMPK. Since glycogen binding inhibits AMPK activity [16], the output of such targeting remains a question mark: either the prevention of the binding would derepress and thus activate the pool of AMPK normally bound inactive at glycogen, or it would mimic the effect of the carbohydrate, possibly by preventing the CBM interaction with the kinase domain [17], and therefore inhibit AMPK. In any case, such strategy could yield interesting compounds and further the understanding of interdependence between AMPK and glycogen. 
In all the cases presented above, isoform-specificity could be taken into account by identification of the key residues that make the difference between isoforms and by taking advantage of this structural information in the rational design strategy (Fig. 4A). Although not all isoform structures have been resolved to date (Fig. 4B), the high sequence identity should allow for homology modeling based on the known ones. The previous paragraphs only referred to structure-based virtual screening but ligandbased screening could also be undertaken for isoform-specific targeting: for instance by using $\alpha 1$-specific C2 versus $\alpha 2$-specific ZMP, or A-769662 and MT47-100 (under the assumption that in each case both compounds bind to the same spot).

In summary, many possibilities remain to target AMPK in novel ways and computeraided techniques show promises that are yet to be taken up.

\section{Concluding remarks}

AMPK is a kinase of utmost interest to the pharmacological field due to its implication in a great number of diseases. While the beneficial effects of AMPK activation have been well-established in several fields, a role for AMPK inhibition in disease treatment is only emerging. The delicate balance between AMPK activation and inhibition forms a challenge for any pharmacotherapeutic strategy that aims at regulation of AMPK activity. At present, no efficient and safe direct targeting of AMPK was achieved but many options are still open. Notably an often declared goal would be to target AMPK in an isoform-dependent manner, and although our knowledge about the different roles of the AMPK complexes still requires deepening, the gathered evidence supports this strategy. A considerable amount of structure-function data has been gathered over the years, but little of it was used for the development of new modulators. The insights these studies gave us could be used for novel strategies and maybe overcome the known shortcomings of currently available modulators. 


\section{References}

1. Viollet, B., Horman, S., Leclerc, J., Lantier, L., Foretz, M., Billaud, M., Giri, S. and Andreelli, F., AMPK inhibition in health and disease. Crit Rev Biochem Mol Biol, 2010. 45(4): p. 276-295.

2. Hardie, D.G., AMP-activated protein kinase: maintaining energy homeostasis at the cellular and whole-body levels. Annu Rev Nutr, 2014. 34: p. 31-55.

3. Canto, C., Jiang, L.Q., Deshmukh, A.S., Mataki, C., Coste, A., Lagouge, M., Zierath, J.R. and Auwerx, J., Interdependence of AMPK and SIRT1 for metabolic adaptation to fasting and exercise in skeletal muscle. Cell Metab, 2010. 11(3): p. 213-219.

4. Samuel, V.T. and Shulman, G.I., Mechanisms for insulin resistance: common threads and missing links. Cell, 2012. 148(5): p. 852-871.

5. Shackelford, D.B. and Shaw, R.J., The LKB1AMPK pathway: metabolism and growth control in tumour suppression. Nature reviews. Cancer, 2009. 9(8): p. 563-575.

6. Rana, S., Blowers, E.C. and Natarajan, A., Small molecule adenosine 5'-monophosphate activated protein kinase (AMPK) modulators and human diseases. J Med Chem, 2015. 58(1): p. 2-29.

7. Xiao, B., Sanders, M.J., Carmena, D., Bright, N.J., Haire, L.F., Underwood, E., Patel, B.R., Heath, R.B., Walker, P.A., Hallen, S., Giordanetto, F., Martin, S.R., Carling, D. and Gamblin, S.J., Structural basis of AMPK regulation by small molecule activators. Nat Commun, 2013. 4: p. 3017.

8. Hardie, D.G., AMPK--sensing energy while talking to other signaling pathways. Cell Metab, 2014. 20(6): p. 939-952.

9. Chen, L., Xin, F.J., Wang, J., Hu, J., Zhang, Y.Y., Wan, S., Cao, L.S., Lu, C., Li, P., Yan, S.F., Neumann, D., Schlattner, U., Xia, B., Wang, Z.X. and Wu, J.W., Conserved regulatory elements in AMPK. Nature, 2013. 498(7453): p. E8-10.

10. Hudson, E.R., Pan, D.A., James, J., Lucocq, J.M., Hawley, S.A., Green, K.A., Baba, O., Terashima, T. and Hardie, D.G., A novel domain in AMP-activated protein kinase causes glycogen storage bodies similar to those seen in hereditary cardiac arrhythmias. Current biology : CB, 2003. 13(10): p. 861-866.

11. Polekhina, G., Gupta, A., Michell, B.J., van Denderen, B., Murthy, S., Feil, S.C., Jennings, I.G., Campbell, D.J., Witters, L.A., Parker, M.W., Kemp, B.E. and Stapleton, D., AMPK beta subunit targets metabolic stress sensing to glycogen. Current biology : CB, 2003. 13(10): p. 867-871.

12. Xiao, B., Heath, R., Saiu, P., Leiper, F.C., Leone, P., Jing, C., Walker, P.A., Haire, L., Eccleston, J.F., Davis, C.T., Martin, S.R., Carling, D. and Gamblin, S.J., Structural basis for AMP binding to mammalian AMP-activated protein kinase. Nature, 2007. 449(7161): p. 496-500.

13. Iseli, T.J., Walter, M., van Denderen, B.J., Katsis, F., Witters, L.A., Kemp, B.E., Michell, B.J. and Stapleton, D., AMP-activated protein kinase beta subunit tethers alpha and gamma subunits via its C-terminal sequence (186-270). J Biol Chem, 2005. 280(14): p. 13395-13400.

14. Wang, S., Song, P. and Zou, M.H., AMP-activated protein kinase, stress responses and cardiovascular diseases. Clin Sci (Lond), 2012. 122(12): p. 555-573.

15. Zmijewski, J.W., Banerjee, S., Bae, H., Friggeri, A., Lazarowski, E.R. and Abraham, E., Exposure to hydrogen peroxide induces oxidation and activation of AMP-activated protein kinase. J Biol Chem, 2010. 285(43): p. 33154-33164.

16. McBride, A., Ghilagaber, S., Nikolaev, A. and Hardie, D.G., The glycogen-binding domain on the AMPK beta subunit allows the kinase to act as a glycogen sensor. Cell Metab, 2009. 9(1): p. 2334.

17. Li, X., Wang, L., Zhou, X.E., Ke, J., de Waal, P.W., Gu, X., Tan, M.H., Wang, D., Wu, D., Xu, H.E. and Melcher, K., Structural basis of AMPK regulation by adenine nucleotides and glycogen. Cell research, 2015. 25(1): p. 50-66.

18. Hegarty, B.D., Turner, N., Cooney, G.J. and Kraegen, E.W., Insulin resistance and fuel homeostasis: the role of AMP-activated protein kinase. Acta Physiol (Oxf), 2009. 196(1): p. 129-145.

19. Chen, Z., Shen, X., Shen, F., Zhong, W., Wu, H., Liu, S. and Lai, J., TAK1 activates AMPK-dependent cell death pathway in hydrogen peroxide-treated cardiomyocytes, inhibited by heat shock protein-70. Mol Cell Biochem, 2013. 377(1-2): p. 35-44.

20. Garcia-Haro, L., Garcia-Gimeno, M.A., Neumann, D., Beullens, M., Bollen, M. and Sanz, P., The PP1R6 protein phosphatase holoenzyme is involved in the glucose-induced dephosphorylation and inactivation of AMP-activated protein kinase, a key regulator of insulin secretion, in MIN6 beta cells. FASEB J, 2010. 24(12): p. 5080-5091.

21. Wang, L. and Brautigan, D.L., alpha-SNAP inhibits AMPK signaling to reduce mitochondrial biogenesis and dephosphorylates Thr172 in AMPKalpha in vitro. Nat Commun, 2013. 4: p. 1559.

22. Wolfson, M., Budovsky, A., Tacutu, R. and Fraifeld, V., The signaling hubs at the crossroad of longevity and age-related disease networks. Int J Biochem Cell Biol, 2009. 41(3): p. 516-520.

23. Moon, S., Han, D., Kim, Y., Jin, J., Ho, W.K. and Kim, Y., Interactome analysis of AMP-activated protein kinase (AMPK)-alpha1 and -beta1 in INS-1 pancreatic beta-cells by affinity purification-mass spectrometry. Sci Rep, 2014. 4: p. 4376.

24. Fogarty, S., Hawley, S.A., Green, K.A., Saner, N., Mustard, K.J. and Hardie, D.G., Calmodulin-dependent protein kinase kinase-beta activates AMPK without forming a stable complex: synergistic effects of Ca2+ and AMP. Biochem J, 2010. 426(1): p. 109-118. 
25. Hardie, D.G., Ross, F.A. and Hawley, S.A., AMPK: $a$ nutrient and energy sensor that maintains energy homeostasis. Nat Rev Mol Cell Biol, 2012. 13(4): p. 251-262.

26. Burkewitz, K., Zhang, Y. and Mair, W.B., AMPKat the nexus of energetics and aging. Cell Metab, 2014. 20(1): p. 10-25.

27. Fogarty, S. and Hardie, D.G., Development of protein kinase activators: AMPK as a target in metabolic disorders and cancer. Biochim Biophys Acta, 2010. 1804(3): p. 581-591.

28. Coughlan, K.A., Valentine, R.J., Ruderman, N.B. and Saha, A.K., AMPK activation: a therapeutic target for type 2 diabetes? Diabetes, metabolic syndrome and obesity : targets and therapy, 2014. 7: p. 241-253.

29. Shirwany, N.A. and Zou, M.H., AMPK in cardiovascular health and disease. Acta Pharmacol Sin, 2010. 31(9): p. 1075-1084.

30. Heydemann, A., The super super-healing MRL mouse strain. Front Biol (Beijing), 2012. 7(6): p. 522-538.

31. Berhanu, T.K., Holley-Cuthrell, J., Roberts, N.W., Mull, A.J. and Heydemann, A., Increased AMPactivated protein kinase in skeletal muscles of Murphy Roth Large mice and its potential role in altered metabolism. Physiol Rep, 2014. 2(3): p. e00252.

32. Price, T.J. and Dussor, G., AMPK: An emerging target for modification of injury-induced pain plasticity. Neurosci Lett, 2013. 557 Pt A: p. 9-18.

33. Bullon, P., Alcocer-Gomez, E., Carrion, A.M., Garrido-Maraver, J., Marin-Aguilar, F., Roman-Malo, L., Ruiz-Cabello, J., Culic, O., Ryffel, B., Apetoh, L., Ghiringhelli, F., Battino, M., Sanchez-Alcazar, J.A. and Cordero, M.D., AMPK phosphorylation modulates pain by activation of NLRP3-inflammasome. Antioxid Redox Signal, 2015. Ahead of print.

34. Carling, D. and Viollet, B., Beyond Energy Homeostasis: the Expanding Role of AMP-Activated Protein Kinase in Regulating Metabolism. Cell Metab, 2015. 21(6): p. 799-804.

35. Kim, M. and Tian, R., Targeting AMPK for cardiac protection: opportunities and challenges. J Mol Cell Cardiol, 2011. 51(4): p. 548-553.

36. Nogueiras, R., Dieguez, C. and Lopez, M., Come to Where Insulin Resistance Is, Come to AMPK Country. Cell Metab, 2015. 21(5): p. 663-665.

37. Jeon, S.M. and Hay, N., The double-edged sword of AMPK signaling in cancer and its therapeutic implications. Arch Pharm Res, 2015. 38(3): p. 346-357.

38. Manwani, B. and McCullough, L.D., Function of the master energy regulator adenosine monophosphate-activated protein kinase in stroke. J Neurosci Res, 2013. 91(8): p. 1018-1029.

39. Brunton, J., Steele, S., Ziehr, B., Moorman, N. and Kawula, T., Feeding uninvited guests: mTOR and AMPK set the table for intracellular pathogens. PLoS Pathog, 2013. 9(10): p. e1003552.

40. Viollet, B., Athea, Y., Mounier, R., Guigas, B., Zar- rinpashneh, E., Horman, S., Lantier, L., Hebrard, S., Devin-Leclerc, J., Beauloye, C., Foretz, M., Andreelli, F., Ventura-Clapier, R. and Bertrand, L., AMPK: Lessons from transgenic and knockout animals. Frontiers in bioscience, 2009. 14: p. 19-44.

41. Laderoute, K.R., Calaoagan, J.M., Madrid, P.B., Klon, A.E. and Ehrlich, P.J., SU11248 (sunitinib) directly inhibits the activity of mammalian 5'-AMP-activated protein kinase (AMPK). Cancer Biol Ther, 2010. 10(1): p. 68-76.

42. Kerkela, R., Woulfe, K.C., Durand, J.B., Vagnozzi, R., Kramer, D., Chu, T.F., Beahm, C., Chen, M.H. and Force, T., Sunitinib-induced cardiotoxicity is mediated by off-target inhibition of AMP-activated protein kinase. Clin Transl Sci, 2009. 2(1): p. 15-25.

43. Hasinoff, B.B., Patel, D. and O'Hara, K.A., Mechanisms of myocyte cytotoxicity induced by the multiple receptor tyrosine kinase inhibitor sunitinib. Mol Pharmacol, 2008. 74(6): p. 17221728.

44. Sinnett, S.E. and Brenman, J.E., Past strategies and future directions for identifying AMP-activated protein kinase (AMPK) modulators. Pharmacology \& therapeutics, 2014. 143(1): p. 111 118.

45. Wu, J., Puppala, D., Feng, X., Monetti, M., Lapworth, A.L. and Geoghegan, K.F., Chemoproteomic analysis of intertissue and interspecies isoform diversity of AMP-activated protein $\mathrm{ki}$ nase (AMPK). J Biol Chem, 2013. 288(50): p 35904-35912.

46. Salt, I., Celler, J.W., Hawley, S.A., Prescott, A., Woods, A., Carling, D. and Hardie, D.G., AMP-activated protein kinase: greater AMP dependence, and preferential nuclear localization, of complexes containing the alpha2 isoform. Biochem $\mathrm{J}$ 1998. 334 ( Pt 1): p. 177-187.

47. Cheung, P.C., Salt, I.P., Davies, S.P., Hardie, D.G. and Carling, D., Characterization of AMP-activated protein kinase gamma-subunit isoforms and their role in AMP binding. Biochem J, 2000. 346 Pt 3: p. 659-669.

48. Koay, A., Woodcroft, B., Petrie, E.J., Yue, H., Emanuelle, S., Bieri, M., Bailey, M.F., Hargreaves, M., Park, J.T., Park, K.H., Ralph, S., Neumann, D. Stapleton, D. and Gooley, P.R., AMPK beta subunits display isoform specific affinities for carbohydrates. FEBS Lett, 2010. 584(15): p. 34993503.

49. Pinter, K., Grignani, R.T., Watkins, H. and Redwood, C., Localisation of AMPK gamma subunits in cardiac and skeletal muscles. Journal of muscle research and cell motility, 2013. 34(5-6): p. 369-378.

50. O'Neill, H.M., Maarbjerg, S.J., Crane, J.D., Jeppesen, J., Jorgensen, S.B., Schertzer, J.D., Shyroka, O., Kiens, B., van Denderen, B.J., Tarnopolsky, M.A., Kemp, B.E., Richter, E.A. and Steinberg, G.R., AMP-activated protein kinase (AMPK) beta1 beta2 muscle null mice reveal an essential role for AMPK in maintaining mitochondrial content 
and glucose uptake during exercise. Proceedings of the National Academy of Sciences of the United States of America, 2011. 108(38): p. 1609216097.

51. Scott, J.W., Galic, S., Graham, K.L., Foitzik, R., Ling, N.X., Dite, T.A., Issa, S.M., Langendorf, C.G., Weng, Q.P., Thomas, H.E., Kay, T.W., Birnberg, N.C., Steinberg, G.R., Kemp, B.E. and Oakhill, J.S., Inhibition of AMP-Activated Protein Kinase at the Allosteric Drug-Binding Site Promotes Islet Insulin Release. Chem Biol, 2015. 22(6): p. 705711.

52. Kim, M., Shen, M., Ngoy, S., Karamanlidis, G., Liao, R. and Tian, R., AMPK isoform expression in the normal and failing hearts. J Mol Cell Cardiol, 2012. 52(5): p. 1066-1073.

53. Fox, M.M., Phoenix, K.N., Kopsiaftis, S.G. and Claffey, K.P., AMP-Activated Protein Kinase alpha 2 Isoform Suppression in Primary Breast Cancer Alters AMPK Growth Control and Apoptotic Signaling. Genes \& cancer, 2013. 4(1-2): p. 3-14.

54. Yun, H. and Ha, J., AMP-activated protein kinase modulators: a patent review (2006 - 2010). Expert Opin Ther Pat, 2011. 21(7): p. 983-1005.

55. Giordanetto, F. and Karis, D., Direct AMP-activated protein kinase activators: a review of evidence from the patent literature. Expert Opin Ther Pat, 2012. 22(12): p. 1467-1477.

56. Sabina, R.L., Patterson, D. and Holmes, E.W., 5-Amino-4-imidazolecarboxamide riboside (Zriboside) metabolism in eukaryotic cells. J Biol Chem, 1985. 260(10): p. 6107-6114.

57. Zhang, Y.L., Guo, H., Zhang, C.S., Lin, S.Y., Yin, Z., Peng, Y., Luo, H., Shi, Y., Lian, G., Zhang, C., Li, M., Ye, Z., Ye, J., Han, J., Li, P., Wu, J.W. and Lin, S.C., AMP as a low-energy charge signal autonomously initiates assembly of AXIN-AMPK-LKB1 complex for AMPK activation. Cell Metab, 2013. 18(4): p. 546-555.

58. Choi, K., Mollapour, E., Choi, J.H. and Shears, S.B., Cellular energetic status supervises the synthesis of bis-diphosphoinositol tetrakisphosphate independently of AMP-activated protein kinase. Mol Pharmacol, 2008. 74(2): p. 527-536.

59. Gomez-Galeno, J.E., Dang, Q., Nguyen, T.H., Boyer, S.H., Grote, M.P., Sun, Z., Chen, M., Craigo, W.A., van Poelje, P.D., MacKenna, D.A., Cable, E.E., Rolzin, P.A., Finn, P.D., Chi, B., Linemeyer, D.L., Hecker, S.J. and Erion, M.D., A Potent and Selective AMPK Activator That Inhibits de Novo Lipogenesis. ACS Med Chem Lett, 2010. 1(9): p. 478-482.

60. Hunter, R.W., Foretz, M., Bultot, L., Fullerton, M.D., Deak, M., Ross, F.A., Hawley, S.A., Shpiro, N., Viollet, B., Barron, D., Kemp, B.E., Steinberg, G.R., Hardie, D.G. and Sakamoto, K., Mechanism of action of compound-13: an alpha1-selective small molecule activator of AMPK. Chem Biol, 2014. 21(7): p. 866-879.

61. Cool, B., Zinker, B., Chiou, W., Kifle, L., Cao, N., Perham, M., Dickinson, R., Adler, A., Gagne, G., Iyengar, R., Zhao, G., Marsh, K., Kym, P., Jung, P., Camp, H.S. and Frevert, E., Identification and characterization of a small molecule AMPK acti- vator that treats key components of type 2 diabetes and the metabolic syndrome. Cell Metab, 2006. 3(6): p. 403-416.

62. Hawley, S.A., Fullerton, M.D., Ross, F.A., Schertzer, J.D., Chevtzoff, C., Walker, K.J., Peggie, M.W., Zibrova, D., Green, K.A., Mustard, K.J., Kemp, B.E., Sakamoto, K., Steinberg, G.R. and Hardie, D.G., The ancient drug salicylate directly activates AMP-activated protein kinase. Science, 2012. 336(6083): p. 918-922.

63. Pang, T., Zhang, Z.S., Gu, M., Qiu, B.Y., Yu, L.F., Cao, P.R., Shao, W., Su, M.B., Li, J.Y., Nan, F.J. and $\mathrm{Li}$, J., Small molecule antagonizes autoinhibition and activates AMP-activated protein kinase in cells. J Biol Chem, 2008. 283(23): p. 1605116060.

64. Li, Y.Y., Yu, L.F., Zhang, L.N., Qiu, B.Y., Su, M.B., Wu, F., Chen, D.K., Pang, T., Gu, M., Zhang, W., Ma, W.P., Jiang, H.W., Li, J.Y., Nan, F.J. and Li, J., Novel small-molecule AMPK activator orally exerts beneficial effects on diabetic $d b / d b$ mice. Toxicol Appl Pharmacol, 2013. 273(2): p. 325-334.

65. Yu, L.F., Li, Y.Y., Su, M.B., Zhang, M., Zhang, W., Zhang, L.N., Pang, T., Zhang, R.T., Liu, B., Li, J.Y., Li, J. and Nan, F.J., Development of Novel Alkene Oxindole Derivatives As Orally Efficacious AMPActivated Protein Kinase Activators. ACS Med Chem Lett, 2013. 4(5): p. 475-480.

66. Jensen, T.E., Ross, F.A., Kleinert, M., Sylow, L., Knudsen, J.R., Gowans, G.J., Hardie, D.G. and Richter, E.A., PT-1 selectively activates AMPKgamma1 complexes in mouse skeletal muscle, but activates all three gamma subunit complexes in cultured human cells by inhibiting the respiratory chain. Biochem J, 2015. 467(3): p. 461472 .

67. Scott, J.W., van Denderen, B.J., Jorgensen, S.B., Honeyman, J.E., Steinberg, G.R., Oakhill, J.S., Iseli, T.J., Koay, A., Gooley, P.R., Stapleton, D. and Kemp, B.E., Thienopyridone drugs are selective activators of AMP-activated protein kinase beta1-containing complexes. Chem Biol, 2008. 15(11): p. 1220-1230.

68. Treebak, J.T., Birk, J.B., Hansen, B.F., Olsen, G.S. and Wojtaszewski, J.F., A-769662 activates AMPK beta1-containing complexes but induces glucose uptake through a PI3-kinase-dependent pathway in mouse skeletal muscle. Am J Physiol Cell Physiol, 2009. 297(4): p. C1041-1052.

69. Wojtaszewski, J.F., Nielsen, P., Hansen, B.F., Richter, E.A. and Kiens, B., Isoform-specific and exercise intensity-dependent activation of 5'-AMPactivated protein kinase in human skeletal muscle. J Physiol, 2000. 528 Pt 1: p. 221-226.

70. Jorgensen, S.B., Viollet, B., Andreelli, F., Frosig, C., Birk, J.B., Schjerling, P., Vaulont, S., Richter, E.A. and Wojtaszewski, J.F., Knockout of the alpha2 but not alpha1 5'-AMP-activated protein kinase isoform abolishes 5-aminoimidazole-4carboxamide-1-beta-4-ribofuranosidebut not contraction-induced glucose uptake in skeletal muscle. J Biol Chem, 2004. 279(2): p. 10701079. 
71. Choi, J., He, N., Sung, M.K., Yang, Y. and Yoon, S., Sanguinarine is an allosteric activator of AMPactivated protein kinase. Biochem Biophys Res Commun, 2011. 413(2): p. 259-263.

72. Zhou, G., Myers, R., Li, Y., Chen, Y., Shen, X., Fenyk-Melody, J., Wu, M., Ventre, J., Doebber, T., Fujii, N., Musi, N., Hirshman, M.F., Goodyear, L.J. and Moller, D.E., Role of AMP-activated protein kinase in mechanism of metformin action. J Clin Invest, 2001. 108(8): p. 1167-1174.

73. Bain, J., Plater, L., Elliott, M., Shpiro, N., Hastie, C.J., McLauchlan, H., Klevernic, I., Arthur, J.S., Alessi, D.R. and Cohen, P., The selectivity of protein kinase inhibitors: a further update. Biochem J, 2007. 408(3): p. 297-315.

74. Machrouhi, F., Ouhamou, N., Laderoute, K., Calaoagan, J., Bukhtiyarova, M., Ehrlich, P.J. and Klon, A.E., The rational design of a novel potent analogue of the 5'-AMP-activated protein kinase inhibitor compound $C$ with improved selectivity and cellular activity. Bioorg Med Chem Lett, 2010. 20(22): p. 6394-6399.

75. Calabrese, M.F., Rajamohan, F., Harris, M.S., Caspers, N.L., Magyar, R., Withka, J.M., Wang, H., Borzilleri, K.A., Sahasrabudhe, P.V., Hoth, L.R., Geoghegan, K.F., Han, S., Brown, J., Subashi, T.A., Reyes, A.R., Frisbie, R.K., Ward, J., Miller, R.A., Landro, J.A., Londregan, A.T., Carpino, P.A., Cabral, S., Smith, A.C., Conn, E.L., Cameron, K.O., Qiu, X. and Kurumbail, R.G., Structural basis for AMPK activation: natural and synthetic ligands regulate kinase activity from opposite poles by different molecular mechanisms. Structure, 2014. 22(8): p. 1161-1172.

76. Phatak, S.S., Stephan, C.C. and Cavasotto, C.N., High-throughput and in silico screenings in drug discovery. Expert opinion on drug discovery, 2009. 4(9): p. 947-959.

77. Kar, S. and Roy, K., How far can virtual screening take us in drug discovery? Expert opinion on drug discovery, 2013. 8(3): p. 245-261.

78. Polishchuk, P.G., Madzhidov, T.I. and Varnek, A., Estimation of the size of drug-like chemical space based on GDB-17 data. Journal of computer-aided molecular design, 2013. 27(8): p. 675679.

79. Clark, D.E., What has virtual screening ever done for drug discovery? Expert opinion on drug discovery, 2008. 3(8): p. 841-851.

80. Sable, R. and Jois, S., Surfing the Protein-Protein Interaction Surface Using Docking Methods: Application to the Design of PPI Inhibitors. Molecules, 2015. 20(6): p. 11569-11603.
81. Sullivan, J.E., Brocklehurst, K.J., Marley, A.E., Carey, F., Carling, D. and Beri, R.K., Inhibition of lipolysis and lipogenesis in isolated rat adipocytes with AICAR, a cell-permeable activator of AMP-activated protein kinase. FEBS Lett, 1994. 353(1): p. 33-36.

82. Fuller, J.C., Burgoyne, N.J. and Jackson, R.M., Predicting druggable binding sites at the proteinprotein interface. Drug discovery today, 2009. 14(3-4): p. 155-161.

83. Sperandio, O., Miteva, M.A., Segers, K., Nicolaes, G.A. and Villoutreix, B.O., Screening Outside the Catalytic Site: Inhibition of Macromolecular Inter-actions Through Structure-Based Virtual Ligand Screening Experiments. The open biochemistry journal, 2008. 2: p. 29-37.

84. Wells, J.A. and McClendon, C.L., Reaching for high-hanging fruit in drug discovery at proteinprotein interfaces. Nature, 2007. 450(7172): p. 1001-1009.

85. Jin, X., Townley, R. and Shapiro, L., Structural insight into AMPK regulation: $A D P$ comes into play. Structure, 2007. 15(10): p. 1285-1295

86. Scholz, R., Suter, M., Weimann, T., Polge, C., Konarev, P.V., Thali, R.F., Tuerk, R.D., Viollet, B., Wallimann, T., Schlattner, U. and Neumann, D., Homo-oligomerization and activation of AMPactivated protein kinase are mediated by the kinase domain alphaG-helix. J Biol Chem, 2009. 284(40): p. 27425-27437.

87. Chapnik, N., Genzer, Y., Ben-Shimon, A., Niv, M.Y. and Froy, O., AMPK-derived peptides reduce blood glucose levels but lead to fat retention in the liver of obese mice. J Endocrinol, 2014. 221(1): p. 89-99.

88. Polekhina, G., Gupta, A., van Denderen, B.J., Feil, S.C., Kemp, B.E., Stapleton, D. and Parker, M.W., Structural basis for glycogen recognition by AMP-activated protein kinase. Structure, 2005. 13(10): p. 1453-1462.

89. Mobbs, J.I., Koay, A., Di Paolo, A., Bieri, M., Petrie, E.J., Gorman, M.A., Doughty, L., Parker, M.W., Stapleton, D., Griffin, M.D. and Gooley, P.R., Determinants of oligosaccharide specificity of the carbohydrate binding modules of AMP-activated protein kinase. Biochem J, 2015. 468(2): p.24557. 


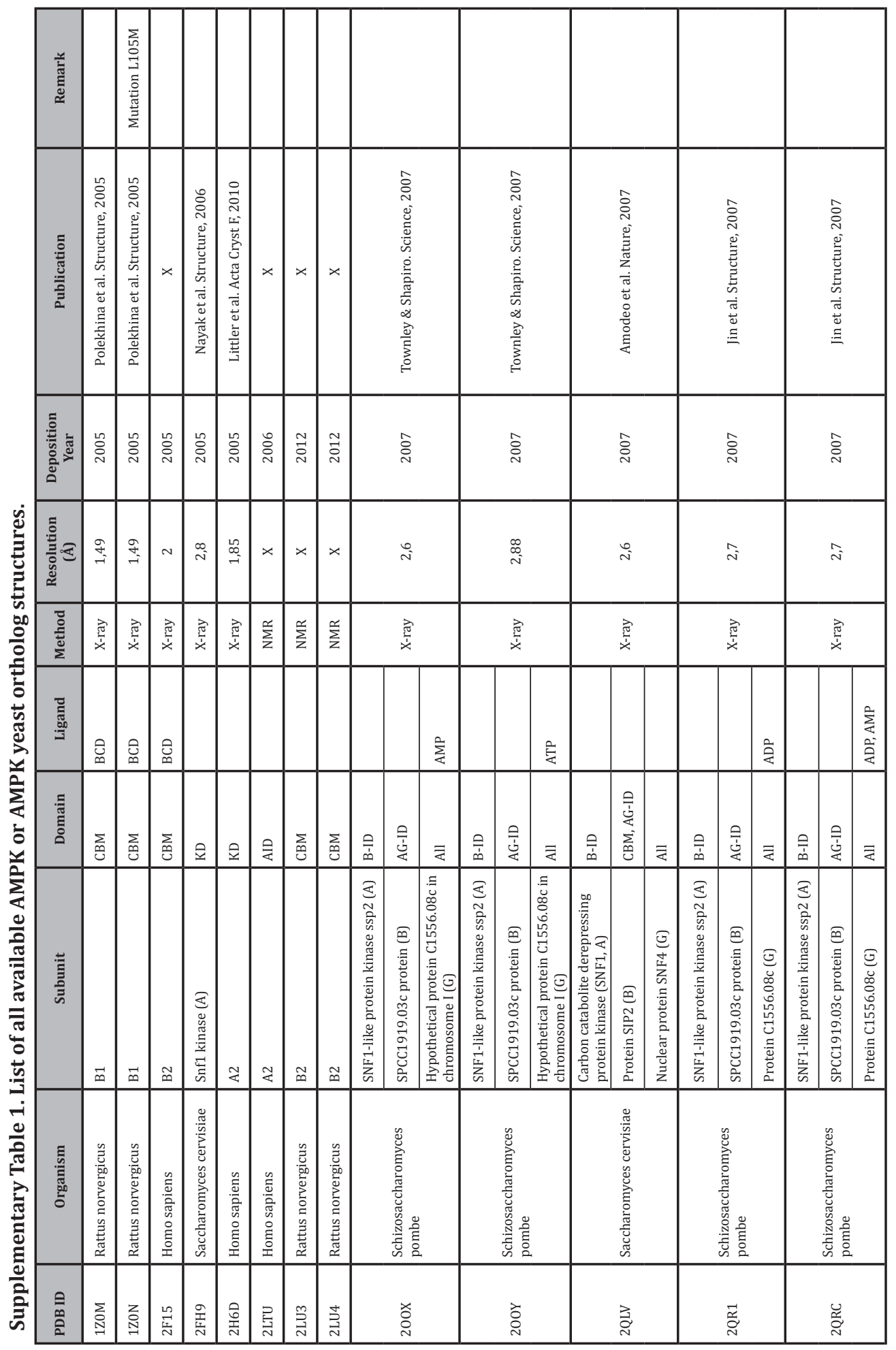




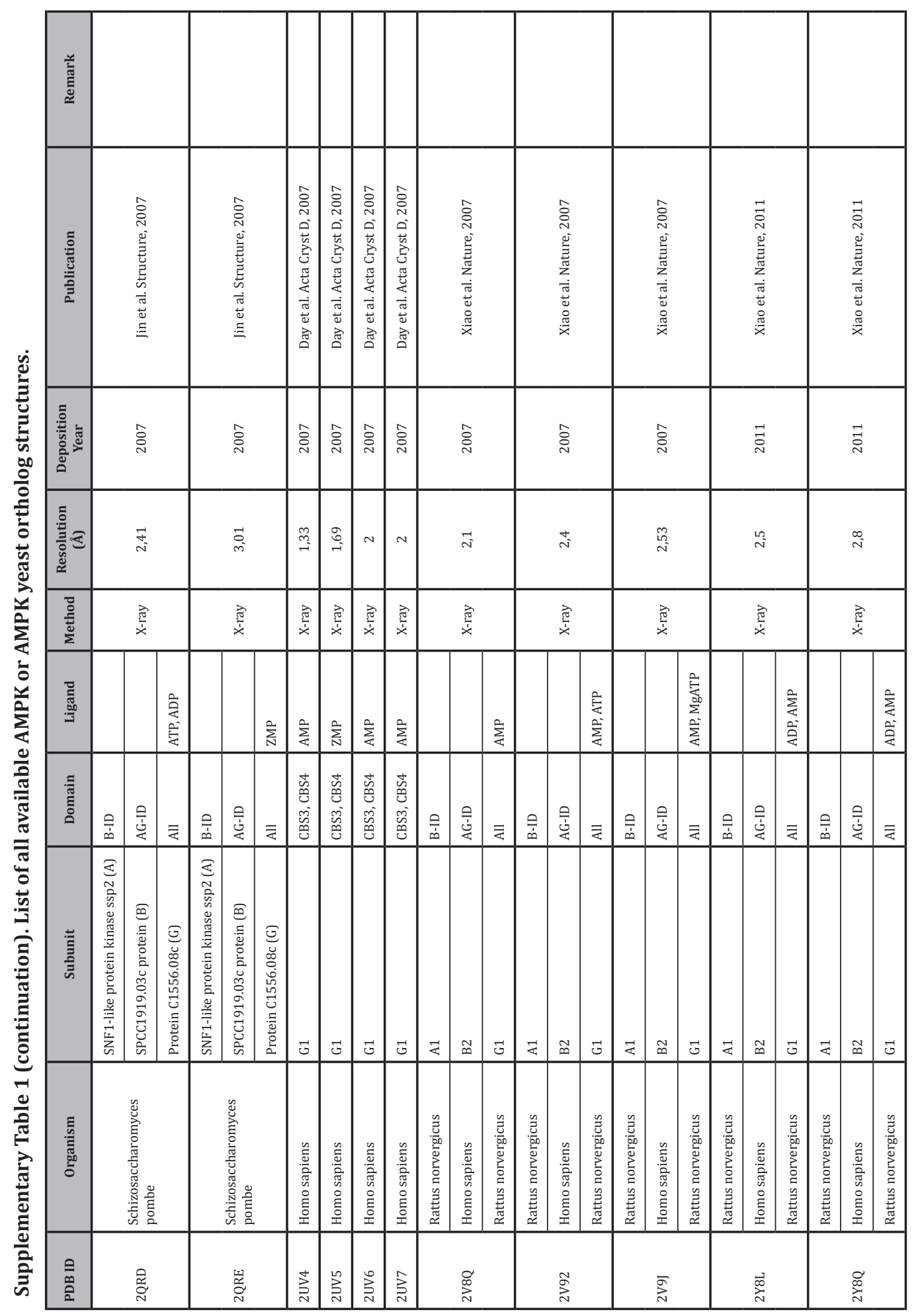




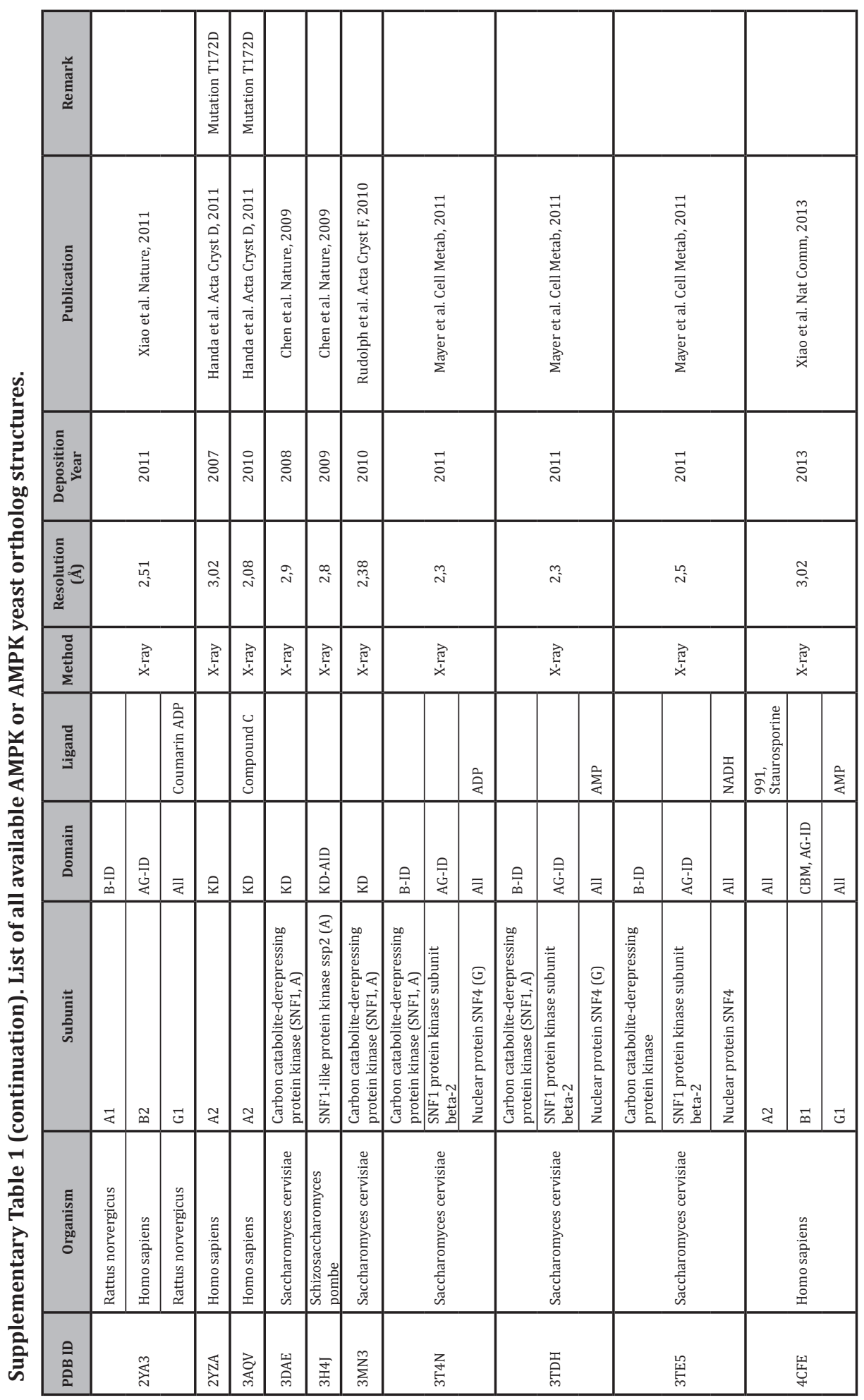




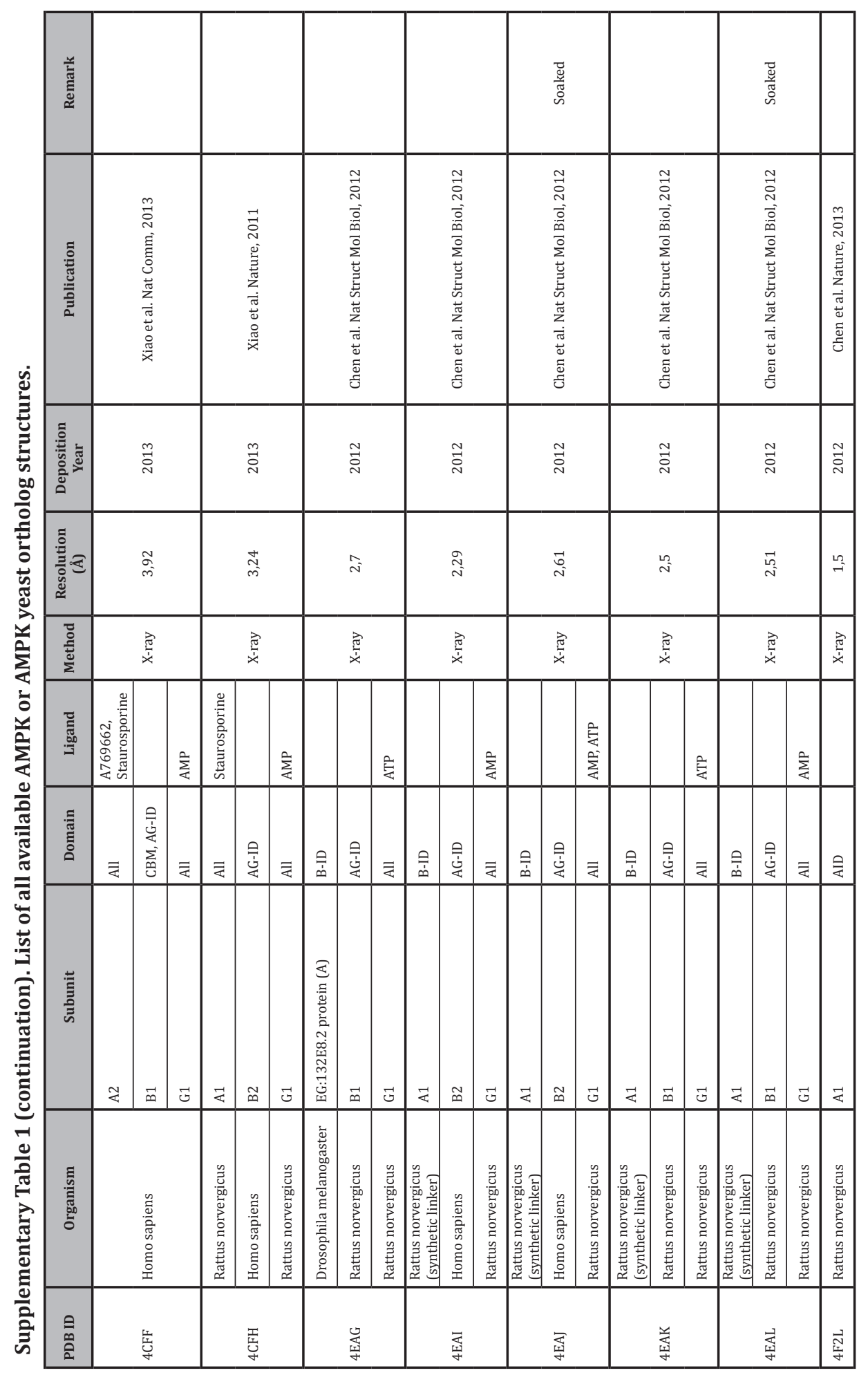




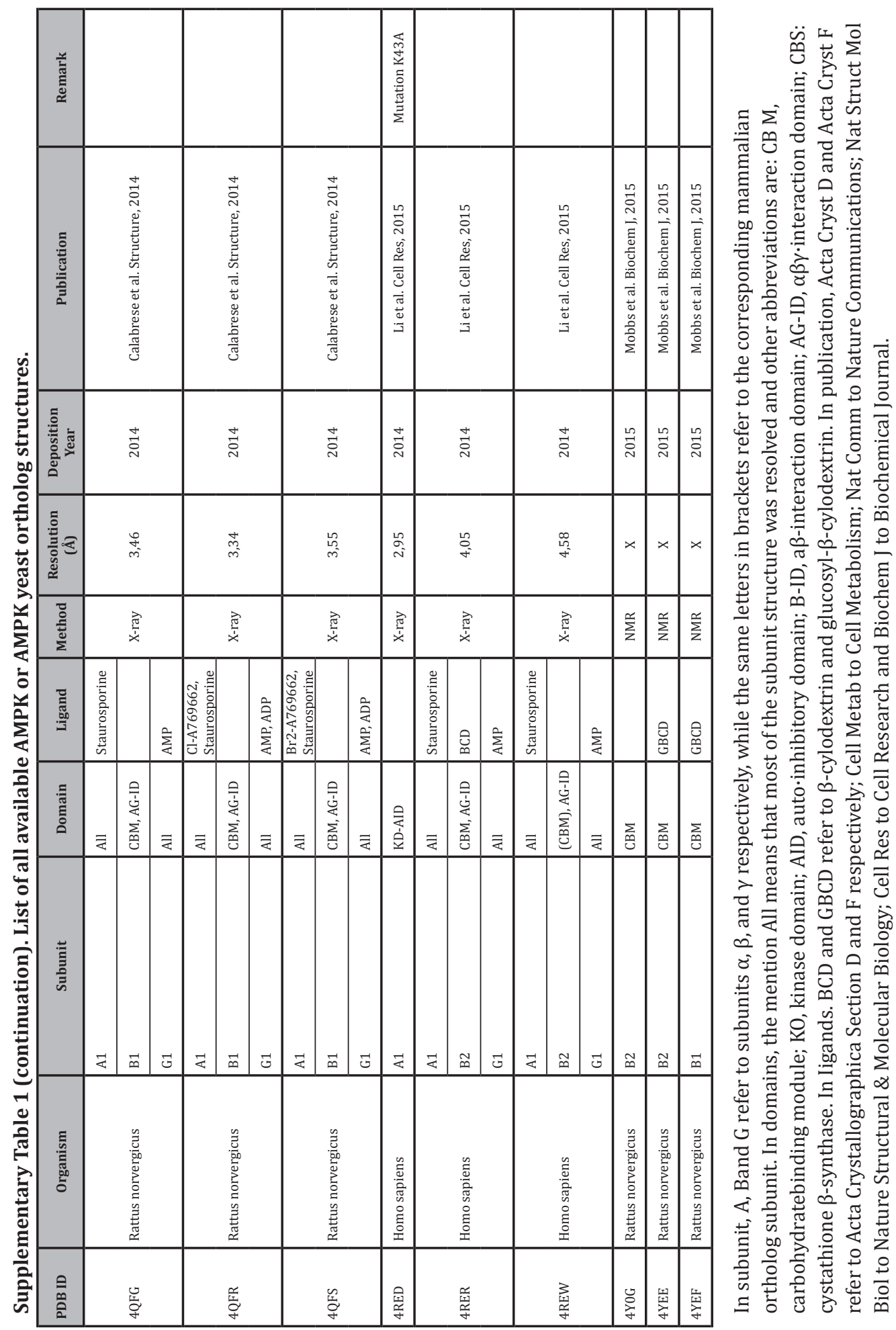





\section{Chapter 3}

\section{The recruitment of AMP-activated protein kinase to glycogen is regulated by autophosphorylation}

Yvonne Oligschlaeger\#, Marie Miglianico\#, Dipanjan Chanda, Roland Scholz, Ramon F. Thali, Roland Tuerk, David I. Stapleton, Paul R. Gooley, Dietbert Neumann

\# equal contribution 


\begin{abstract}
The mammalian AMP-activated protein kinase (AMPK) is an obligatory $\alpha \beta \gamma$ heterotrimeric complex carrying a carbohydrate-binding module (CBM) in the $\beta$-subunit (AMPK $\beta$ ) capable of attaching AMPK to glycogen. Nonetheless, AMPK localizes at many different cellular compartments, implying the existence of mechanisms that prevent AMPK from glycogen binding. Cell-free carbohydrate binding assays revealed that AMPK autophosphorylation abolished its carbohydrate-binding capacity. X-ray structural data of the CBM displays the central positioning of threonine-148 residue (T148) within the binding pocket. Substitution of T148 for a phosphomimicking aspartate (T148D) prevents AMPK from binding to carbohydrate. Overexpression of isolated CBM or $\beta 1$-containing AMPK in cellular models revealed that wild-type (WT) localizes to glycogen particles, whereas T148D shows a diffuse pattern. Pharmacological AMPK activation and glycogen degradation by glucose deprivation but not forskolin enhanced cellular T148 phosphorylation. Cellular glycogen content was higher if pharmacological AMPK activation was combined with overexpression of T148D mutant relative to WT-AMPK. In summary, these data show that glycogen-binding capacity of AMPK $\beta$ is regulated by T148 autophosphorylation with likely implications in regulation of glycogen turnover. The findings further raise the possibility of regulated carbohydrate-binding function in a wider variety of CBMcontaining proteins.
\end{abstract}




\section{Introduction}

Many enzymes involved in the synthesis and degradation of complex carbohydrate molecules, such as cellulose or glycogen, contain a domain called the carbohydratebinding module (CBM) specialized in the binding of complex carbohydrates. CBMs are present in various taxa and protein types [1,2]. Despite their lack of direct catalytic activity, CBMs may enhance specific enzymatic activity by targeting the enzyme to its substrates and increasing its effective concentration [3,4]. Other proteins that are not directly carbohydrate-active but are involved in glucose metabolism regulation are also known to contain a CBM: this is notably the case for the mammalian energy sensor AMP-activated protein kinase (AMPK) [5, 6], as well as its fungal homolog SNF1 [7] and its plant homolog SnRK1 [8].

AMPK is a heterotrimer consisting of (i) a catalytic subunit $\alpha$ ( $\alpha 1$ or $\alpha 2$ ) carrying the kinase domain, (ii) a regulatory subunit $\beta$ ( $\beta 1$ or $\beta 2$ ) with the CBM and the C-terminal region tethering $\alpha$ and $\gamma$ together, and (iii) a regulatory subunit $\gamma(\gamma 1, \gamma 2$ or $\gamma 3)$ responsible for the adenine nucleotide sensing $[9,10]$. AMPK is a well-known, highly conserved metabolic enzyme central for coordinating cellular and whole body energy homeostasis. Upon cellular stress, such as contraction, exercise or hypoxia, AMPK is activated by several mechanisms. Briefly, the increased AMP level induces a conformational change in AMPK facilitating the phosphorylation of threonine-172 (T172) on the activation loop of the $\alpha$-subunit by upstream kinases such as liver kinase B1 (LKB1) or calcium/calmodulin-dependent protein kinase 2 (CaMKK2) resulting in roughly 1000-fold activation of the enzyme [11-13]. The AMP-binding to AMPK $\gamma$ also reduces the rate of dephosphorylation at T172, thus keeping AMPK in its active state $[11,14]$. As a result, AMPK phosphorylates a number of downstream targets to restore energy balance by facilitating glucose uptake, glycolysis, and fatty acid oxidation, thus increasing the energy production, and simultaneously switching off ATP-consuming pathways, such as glycogen, fatty acid and cholesterol synthesis $[9,12]$.

Considering the micromolar affinity of recombinant AMPK complexes and CBM for small cyclic and linear oligosaccharides $[15,16]$, the interaction between AMPK and glycogen in cells may be relatively strong. Since some AMPK targets, such as glycogen synthase [17], are associated with glycogen [18], the presence of a CBM might be beneficial in juxtaposing the kinase next to its substrates [5]. However, in the case of targets not located at glycogen, such as the acetyl-CoA carboxylase 2 (ACC2), which is associated with the mitochondria [19], the retention of AMPK to glycogen by the CBM could be detrimental for a quick cellular response to energy stress. Besides, AMPK has been detected in different subcellular areas, for example at the cell membrane [20,21] or in the nucleus $[22,23]$. This variety of localizations and actions implies that the 
AMPK-carbohydrate-binding ability may be regulated in cells.

In this study, using cell-free and cellular systems, we show that AMPK loses its carbohydrate-binding ability upon activation due to a bimolecular autophosphorylation event that modifies the AMPK $\beta$-CBM at threonine-148 (T148).

\section{Experimental procedures}

\section{Plasmids}

Bacterial expression plasmids encoding for hexahistidine-tagged wild-type (WT) AMPK $(\alpha 1 \beta 1 \gamma 1, \alpha 1 \beta 2 \gamma 1, \alpha 2 \beta 1 \gamma 1, \alpha 2 \beta 2 \gamma 1)[24,25]$, non-tagged LKB1-M025-STRAD $\alpha$ complex [26], and GST-tagged CaMKK2 [11] have been described. The isolated $\beta 1$ - and $\beta 2$-CBMs were bacterially expressed as described [16]. The cDNA of $\beta 1$ lacking the CBM (aa 182-270) was amplified by PCR and initially subcloned into the NcoI and SpeI restriction sites of the pET3dx vector [24]. Subsequently, the bacterial expression plasmid encoding for the heterotrimeric hexahistidine-tagged AMPK lacking the CBM $(\alpha 1 \beta 1 \Delta \mathrm{CBM} \gamma 1)$ was generated using the published procedure [24]. The kinasedeficient AMPK $\alpha 1$ plasmids with a non-phosphorylatable alanine (D157A) $(\alpha 1 \beta 1 \gamma 1$ KD or $\alpha 1 \beta 2 \gamma 1-\mathrm{KD}$, respectively) were constructed as published earlier [27]. The coding sequence for T148 in the AMPK $\beta 1$ subunits was changed to encode a nonphosphorylatable alanine (T148A) or a phosphorylation-mimicking aspartate (T148D) by site-directed mutagenesis following the manufacturer's instructions (QuikChange, Stratagene).

To obtain activated AMPK phosphorylated at T172, each of the tricistronic WT and kinase-deficient coding sequences were combined with the LKB1-MO25-STRAD $\alpha$ coding sequence in a single hexacistronic plasmid, allowing for co-expression of both protein complexes and the subsequent purification of the hexahistidine-tagged AMPK alone (referred to as $\alpha 1 \beta 1 \triangle \mathrm{CBM} \gamma 1$ active, $\alpha 1 \beta 1 \gamma 1$ and $\alpha 1 \beta 2 \gamma 1$ active, $\alpha 1 \beta 1 \gamma 1-\mathrm{KD}$ pT172, and T148A active, respectively) as also published recently [28].

For expression in mammalian cells, the cDNA of wild-type (WT) $\beta 1$-CBM (aa 68163 ) or $\beta 2$-CBM (aa 67-163) was amplified by PCR and ligated in frame into the pAcGFP expression vector (Clontech) via the EcoRI and SalI restriction sites, resulting in expression constructs for CBM $\beta 1$-WT-GFP or CBM $\beta 2$-WT-GFP, respectively. The cDNA of full-length $\beta 1$ was amplified by PCR and subcloned either into the pmCherry expression vector (Clontech) via EcoRI and SalI restriction sites, or into the pCMV53xHA expression vector [29] via HindIII and SalI restriction sites. GFP-tagged CBMs or mCherry-tagged $\beta 1$ bearing a threonine-to-aspartate mutation on residue 148 (T148D) were generated using the Quick-change site-directed mutagenesis kit (Stratagene). The pcDNA3 constructs for expression of AMPK $\alpha 1$-myc and $\gamma 1$-subunit were kindly 
provided by Dr. D. Carling (Imperial College London, London, United Kingdom).

For retroviral overexpression, GFP-tagged CBM $\beta 1$ or CBM $\beta 2$ (either WT or T148D) was subcloned, using an oligonucleotide linker, into the EcoRI and SalI restriction sites of the pBMZ-ires-neo retroviral backbone (kindly provided by Dr. G. Nolan, Stanford University, CA). Primer sequences are available from the author upon request. All of the constructs were verified by sequencing.

\section{Bacterial Expression and Purification}

Proteins were expressed in Rosetta 2 (DE3) Escherichia coli cells (Novagen). Bacteria were grown in auto-induction medium and proteins were purified as described with few modifications [30]. Briefly, the bacterial pellet was collected by centrifugation, resuspended in lysis buffer (for hexahistidine-tagged proteins: $50 \mathrm{mM} \mathrm{NaH}_{2} \mathrm{PO}_{4}, 30 \%$ Glycerol, 0.5 M sucrose, 10 mM imidazole, pH 8; for GST-tagged proteins: PBS, pH 7.3) and lysed using a high-pressure homogenizer. After centrifugation, the supernatant was incubated for 1 hour at $4^{\circ} \mathrm{C}$ on a roller bank in presence of either $1 \mathrm{~mL}$ of nickelsepharose HP (GE Healthcare) for the hexahistidine-tagged proteins or with $1 \mathrm{~mL}$ of gluthathione sepharose 4B (GE Healthcare) for the GST-tagged proteins. After centrifugation, the resin was washed 3 times with wash buffer (for hexahistidinetagged proteins: $50 \mathrm{mM} \mathrm{NaH}_{2} \mathrm{PO}_{4}, 30 \%$ Glycerol, $0.5 \mathrm{M}$ sucrose, $20 \mathrm{mM}$ imidazole, $\mathrm{pH}$ 8; for GST-tagged proteins: PBS, pH 7.3) then the protein was eluted in elution buffer (for hexahistidine-tagged proteins: $50 \mathrm{mM} \mathrm{NaH} \mathrm{PO}_{4}, 30 \%$ Glycerol, $0.5 \mathrm{M}$ sucrose, $250 \mathrm{mM}$ imidazole, pH 8; for GST-tagged proteins: Tris-HCl $50 \mathrm{mM}, 10 \mathrm{mM}$ reduced glutathione, $\mathrm{pH} 8$ ), frozen in liquid nitrogen and kept at $-80^{\circ} \mathrm{C}$ until use.

\section{$\beta$-cyclodextrin Binding Assay}

$\beta$-cyclodextrin was immobilized on epoxy-activated sepharose 6B (GE Healthcare) according to the manufacturer's protocol. Briefly, $\mathrm{Ni}^{2+}$-affinity purified AMPK was rebuffered into assay buffer (10 mM Tris- $\mathrm{HCl} \mathrm{pH}$ 7.2) using PD-10 columns (GE Healthcare) and $500 \mu \mathrm{l}(25 \mu \mathrm{g} / \mathrm{ml})$ of this AMPK solution (I-fraction) was incubated with $50 \mu \mathrm{l}$ of pre-equilibrated $\beta$-cyclodextrin resin in a $0.7 \mathrm{ml}$ reaction tube (LoBind, Eppendorf) with gentle agitation for 15-20 minutes. The supernatant containing the non-bound material (S-fraction) was removed after centrifugation (2000 $\times$ g, $1 \mathrm{~min}$ ) and the resin was washed twice in assay buffer. The wash fractions were discarded. Bound AMPK was eluted by incubation in $500 \mu$ of elution buffer $(5 \mathrm{mM} \beta$-cyclodextrin in assay buffer) for 5 min with gentle agitation. After centrifugation the supernatant (P-fraction) was collected, the resin was washed twice with elution buffer and supernatants were discarded. Finally, the resin was incubated with SDS-sample buffer at $95^{\circ} \mathrm{C}$ to obtain the precipitated fraction (L-fraction). For analyses, $5 \mu$ l of each fraction 
were subjected to SDS-PAGE and Western blotting, and further processed with AMPK $\alpha$ and phospho-AMPK (pT172) antibodies (Cell Signaling Technologies).

\section{Phosphorylation Assay of Recombinant Proteins}

AMPK was in-vitro activated by upstream kinases as previously described [30]. Briefly, recombinant AMPK $(\alpha 1 \beta 1 \gamma 1$ or $\alpha 1 \beta 2 \gamma 1,25 \mu \mathrm{g} / \mathrm{ml})$ was activated in kinase buffer by recombinant GST-CamKK2 $(15 \mu \mathrm{g} / \mathrm{ml})$ for $30 \mathrm{~min}$ at $37^{\circ} \mathrm{C}$, and subsequently incubated with $\beta$-cyclodextrin resin for $10 \mathrm{~min}$ with gentle agitation to allow for binding. Alternatively, recombinant AMPK was first incubated with $\beta$-cyclodextrin resin, followed by activation with upstream kinases. As described above, the assay was continued with three washing steps, then three times $\beta$-cyclodextrin elution buffer, and finally SDS-sample buffer. Subsequently, the presence of (phosphorylated) AMPK in the various fractions (I, S, P, L) was probed by Western blot analysis.

\section{Cell Culture}

The human hepatocyte HepG2 and human embryonic kidney 293T cell line (Hek293T) were cultured in DMEM with high glucose (25 mM) (Gibco), supplemented with $10 \%(\mathrm{v} / \mathrm{v})$ heat-inactivated fetal calf serum (Bodinco BV, Alkmaar, The Netherlands) and penicillin/streptomycin (Invitrogen), unless otherwise stated. HL-1 cardiomyocyte cell line was kindly provided by Dr. W. Claycomb (Louisiana State University, New Orleans, LA, USA), cultured on fibronectin (5 ug/ml, Sigma)/gelatin (0.01\%, Merck)-coating in Claycomb medium (supplemented with $10 \%$ heatinactivated fetal calf serum (iFCS), $0.1 \mathrm{mmol} / \mathrm{l}$ noradrenaline [norepinephrine], $2 \mathrm{mmol} / \mathrm{l} \mathrm{L-glutamine}, 100 \mathrm{U} / \mathrm{ml}$ penicillin and $100 \mu \mathrm{g} / \mathrm{ml}$ streptomycin) at $37^{\circ} \mathrm{C}$ and $5 \%$ CO2.

For transient transfections, Hek293T cells were seeded to $30 \%$ confluence in 6-well plates (Greiner Bio-one) $24 \mathrm{~h}$ before transfection. Cells were co-transfected with plasmid DNA ( $\alpha 1$-myc, mCherry-tagged $\beta 1$-WT/ $\beta 1-\mathrm{T} 148 \mathrm{D}$ and $\gamma 1$ for immunoprecipitation and Western blotting, or $\alpha 1$-myc, HA-tagged $\beta 1, \gamma 1$ and mCherrytagged $\beta 1-\mathrm{WT} / \beta 1-\mathrm{T} 148 \mathrm{D}$ for localization studies of the holoenzyme) using Lipofectamine 2000 (Invitrogen) in antibiotic-free culture medium. Six to eight hours after transfection, transfection medium was replaced by normal growth medium. At 24 - 48h after transfection, cells were either harvested or fixed.

For the glycogen depletion experiments, cells were either maintained in high glucose medium (DMEM with $25 \mathrm{mM}$ glucose and 10\% iFCS) or treated with forskolin $(100 \mu \mathrm{M}$, Sigma) in the same medium, or glucose-deprived (glucose-free DMEM, 10\% iFCS), for 16 hours. 
In order to activate cellular AMPK, cells were serum-starved (plain DMEM, 5.5 mM glucose) for $16 \mathrm{~h}$ and subsequently treated with 5-aminoimidazole-4-carboxamide riboside (AICAR, 1 or $1.5 \mathrm{mM}$, Sigma), Oligomycin (3 or $5 \mu \mathrm{M}$, Sigma), A769662 (100 $\mu \mathrm{M}$ ) or phenformin (1.5 mM), vehicle (DMSO) or high glucose medium for $45-60 \mathrm{~min}$. Insulin (100 nM, Sigma) treatment for $15 \mathrm{~min}$ was done in order to stimulate glycogen synthesis.

\section{Immunoprecipitation and Immunoblotting}

Cells were lysed in immunoprecipitation lysis buffer $(20 \mathrm{mM}$ Tris- $\mathrm{HCl} \mathrm{pH}$ 8.0, $137 \mathrm{mM} \mathrm{NaCl}, 10 \%$ glycerol, $1 \%$ Triton X-100, 2 mM EDTA), supplemented with protease and phosphatase inhibitor cocktails (Roche). Endogenous AMPK was immunoprecipitated using a combination of AMPK $\alpha 1$ and AMPK $\alpha 2$ antibodies raised in sheep (kindly provided by G. Hardie). Myc-tagged AMPK was immunoprecipitated using a myc-tag antibody (Cell Signaling Technology, Beverly, MA). The primary antibody was incubated top-over-top with $350 \mathrm{ug}$ of protein lysate at $4{ }^{\circ} \mathrm{C}$ for $16 \mathrm{~h}$, followed by incubation with protein G Sepharose beads for $3-4 \mathrm{~h}$ at $4{ }^{\circ} \mathrm{C}$. The immune complexes were then collected by centrifugation. The immunoprecipitated proteins were electrophoresed by SDS-PAGE and analyzed by Western blot analysis. Immunoblot analysis was carried out with the following primary antibodies: myc-tag, AMPK $\alpha$, phospho-AMPK-T172, Akt, phospho-Akt-S473 and GS (all from Cell Signaling). GS-pS7 antibodies were a kind gift from G. Hardie. In order to detect changes in T148 phosphorylation, a phospho-specific AMPK $\beta$-T148 antibody was produced (peptide sequence AMPK $\beta 2$ (142-154) pT148; VTSQLGINNLI) [5]. Detection was performed using anti-rabbit or anti-mouse horse-radish peroxidase (HRP)-conjugated secondary antibodies (Cell Signaling Technology or Dako, respectively), followed by chemiluminescence.

\section{Biochemical Cellular Glycogen Measurement}

Extraction of glycogen from 293T cells was adapted from a method described by McMahon and Frost [31]. Briefly, cells were lysed in potassium hydroxide (30\%) and boiled at $70^{\circ} \mathrm{C}$ for $30 \mathrm{~min}$. Subsequently, samples were cooled to $25^{\circ} \mathrm{C}$ before sodium sulfate $(6 \% \mathrm{w} / \mathrm{v})$ and $\mathrm{EtOH}(99.5 \% \mathrm{v} / \mathrm{v})$ were added at the ratio 1:1:3. After thorough mixing, samples were rotated top-over-top at $4^{\circ} \mathrm{C}$ for $30-60 \mathrm{~min}$. The precipitate was collected by centrifugation at $5000 \mathrm{rpm}$ for $5 \mathrm{~min}$ at $4^{\circ} \mathrm{C}$. To hydrolyze, pellets were dissolved in $1 \mathrm{M} \mathrm{HCl}$ and boiled at $100^{\circ} \mathrm{C}$ for $2 \mathrm{~h}$. Samples were cooled before neutralization using $2 \mathrm{M} \mathrm{NaOH}$. Hydrolysates were used for glucose determination using a Glucose (GO) Assay Kit (Sigma), according to the manufacturer's instructions. 


\section{Retroviral Vectors and Infections}

Retroviral systems and Phoenix helper-free retrovirus producer cell lines were used as published before [32-34]. Amphotropic retroviral supernatants were produced following calcium phosphate/DNA transfection of producer cells; 24-48 hours posttransfection, the supernatants were harvested, filtered ( 0.45 micron filters; Corning, Germany) and used for infection of HepG2, Hek293T and HL-1 cells in presence of $4 \mu \mathrm{g} / \mathrm{ml}$ polybrene (Sigma). For infections, cells were incubated with virus particles for 6-8 hours and then allowed to recover for 48 hours on fresh medium before selection pressure was applied. Infected cells were selected with 200 to $500 \mu \mathrm{g} / \mathrm{ml} \mathrm{G418} \mathrm{(PAA}$ Laboratories $\mathrm{GmbH}$ ) for two weeks preceding experiments.

\section{Immunocytochemistry}

Cells were grown in 12-wells plates (Greiner Bio-One) on coverslips (Ø $20 \mathrm{~mm}$; Thermo Scientific) to 60-80 \% confluence. Cells were washed twice with PBS and fixed with $4 \%$ formaldehyde in PBS for $10 \mathrm{~min}$ at room temperature. Fixed cells were stored at $4{ }^{\circ} \mathrm{C}$ in PBS-NaN ${ }_{3}(0.03 \%)$ or washed three times with PBS+/+ (Gibco) and directly used for immunocytochemistry (IC). Subsequently, cells were permeabilized $(0.1 \%$ Triton X-100 and 0.2 \% BSA in PBS) for $15 \mathrm{~min}$ and blocked (2 \% BSA-PBS) for $30 \mathrm{~min}$ at room temperature. Primary and secondary antibodies ( $2 \%$ BSA-PBS) were incubated for $1 \mathrm{~h}$ at room temperature. Coverslips were washed and mounted onto glass slides using DABCO-glycerol medium (Sigma-Aldrich) containing diamidino-2-phenylindole (DAPI, 1:10000, Sigma-Aldrich) in order to counterstain nuclei. The anti-glycogen antibody (1:500) was a courtesy of Dr. O. Baba (Tokyo Medical and Dental University, Tokyo, Japan), the secondary antibody was goat anti-mouse IgM Alexa647 (1:200, Invitrogen) or goat anti-mouse IgM Alexa488 (1:200, Invitrogen).

\section{Microscopy and Image Capturing}

Fixed cells were imaged using a Leica TCS SPE confocal laser scanning microscope (Leica Microsystems $\mathrm{GmbH}$ ) equipped with an air-cooled Argon-Krypton mixed gas laser, using oil-immersion objectives $(63 \times, N A=1.4)$. Optical sections were recorded 3 scans for each image. Image J software was used to process and analyze the images. Image brightness and contrast was adjusted to the same settings where needed.

\section{Statistical Analysis}

All bar graph data are presented as means \pm SEM. Statistical analysis was performed by using Student's $t$-test and statistical analysis software Prism 4 (GraphPad Software, Inc.). A p-value of $<0.05$ was considered statistically significant. 


\section{Results}

\section{The carbohydrate-binding ability of AMPK is lost upon activation}

To verify the carbohydrate-binding ability of AMPK, $\beta$-cyclodextrin was coupled to sepharose beads, and recombinant AMPK $(\alpha 1 \beta 1 \gamma 1)$ was expressed in bacteria, purified and loaded onto the packed column. As shown in Figure 1A, immobilized $\beta$-cyclodextrin retains the AMPK complex in the column and could therefore be used to affinity-purify recombinant AMPK. On the contrary, when no $\beta$-cyclodextrin was immobilized to the sepharose, the overexpressed AMPK was not retained confirming that AMPK retention is dependent on $\beta$-cyclodextrin. In further experiments, this procedure was simplified by allowing the recombinant AMPK to bind to the $\beta$-cyclodextrin resin in a batch set-up (Fig. 1B) instead of using a column. Fractions of the protein that could not bind to the resin stayed in the supernatant $(S)$, whereas fractions that bound to the resin were eluted from this initial pellet fraction (P) by inclusion of soluble $\beta$-cyclodextrin in the buffer. The remaining resin bound proteins were solubilized by extraction with Laemmli buffer (L) and considered as unspecific precipitates. All fractions were collected and analyzed by SDS-PAGE followed by Coomassie Brilliant Blue Stain or Western blotting. This assay was performed both with the isolated CBM ( $\beta 1$-CBM or $\beta 2$-CBM, respectively) and a kinase-dead (KD) mutant of AMPK ( $\alpha 1 \beta 1 \gamma 1-\mathrm{KD}$ or $\alpha 1 \beta 2 \gamma 1-$ KD, respectively) (Fig. 1C). As expected, both CBM isoforms as well as both catalytically inactive AMPK complexes were able to bind to the resin and were consequently found in the pellet fraction.

To obtain T172-phosphorylated active AMPK, the tricistronic plasmid for AMPK expression [24,25] was combined with the LKB1-Mo25 $\alpha$-Strad $\alpha$ tricistron resulting in a hexacistronic AMPK-LKB1 co-expression construct (Fig. 1D). AMPK $\alpha$ is the only protein of the plasmid constructs carrying a His-tag, which resulted in purified AMPK complexes when using the His-tag purification protocol. Heterotrimeric $\alpha 1 \beta 1 \gamma 1$ and $\alpha 1 \beta 2 \gamma 1$ AMPK complexes, as well as the AMPK $\alpha 1 \beta 1 \gamma 1$ complex lacking the N-terminal CBM $(\alpha 1 \beta 1-\triangle \mathrm{CBM} \gamma 1)$, were expressed and purified in its inactive and active state (Fig. 1E). As shown in Figure 1F, the $\alpha 1 \beta 1-\triangle \mathrm{CBM} \gamma 1$ complex was expectedly found in the supernatant fraction and therefore did not retain the ability to bind to carbohydrates (Fig. 1F, upper panel). As seen before with the KD mutants, the inactive forms of the AMPK complexes containing full-length $\beta 1$ or $\beta 2$ normally bound to $\beta$-cyclodextrin, since these were found in the pellet fraction. In contrast, the active AMPK isoforms showed a loss of binding-affinity to the $\beta$-cyclodextrin resin similar to the truncated AMPK complex (Fig 1F, lower panel). These findings suggest that activation of AMPK prevents its later binding to carbohydrates such as $\beta$-cyclodextrin. 

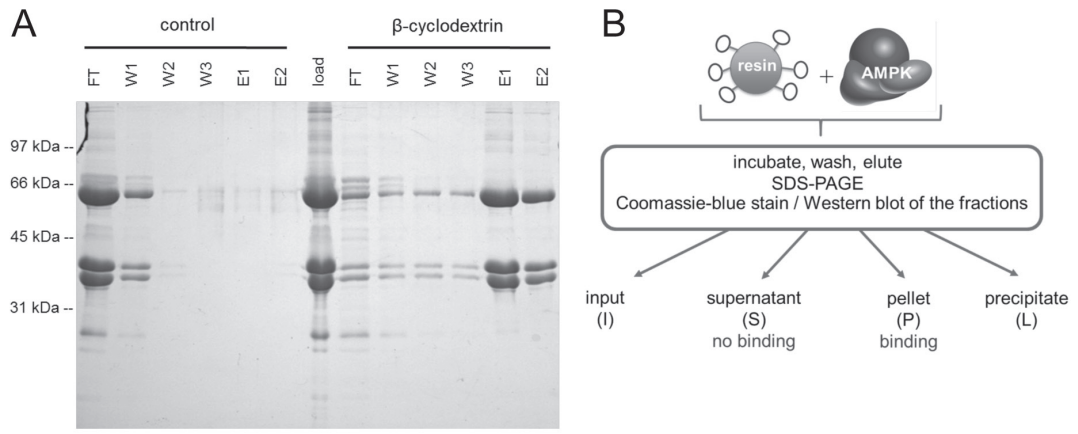

C
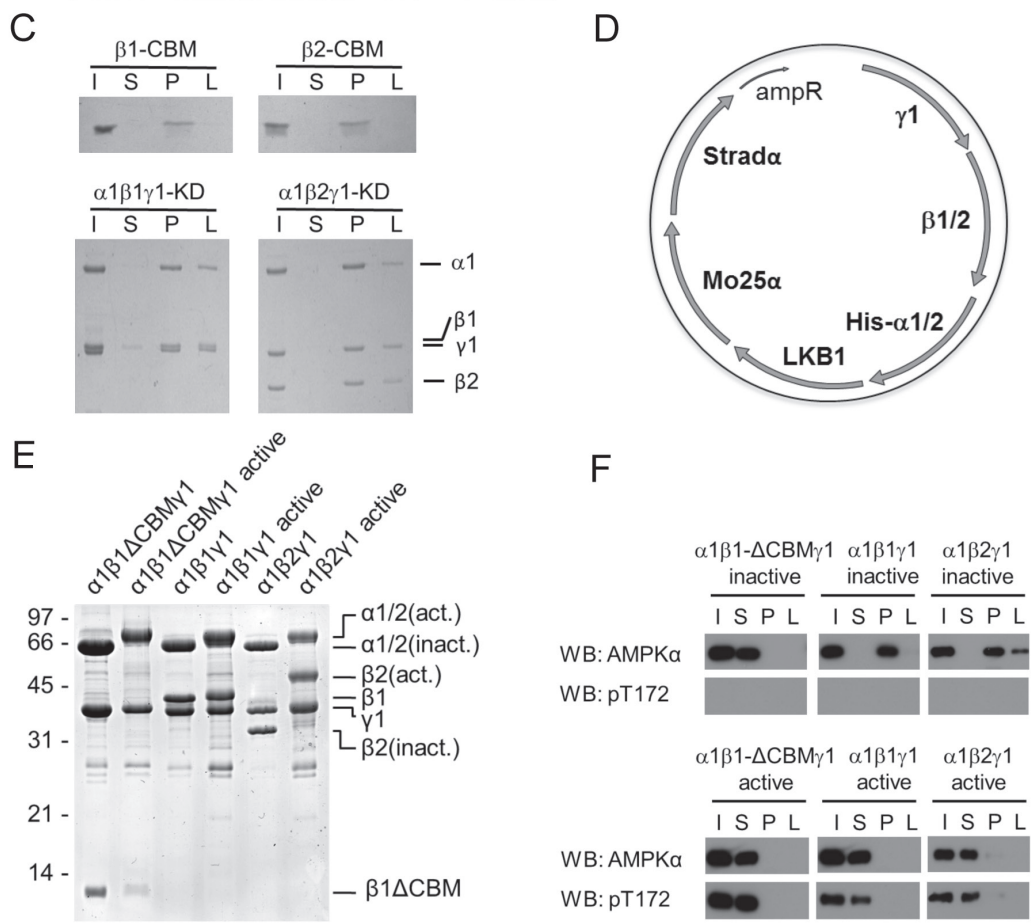

Figure 1. The carbohydrate-binding ability of AMPK is lost upon activation.

(A) Application of immobilized $\beta$-cyclodextrin for purification of AMPK. Epoxy-activated Sepharose was allowed to react with soluble $\beta$-cyclodextrin or was inactivated (control). The column resin was equilibrated in $100 \mathrm{mM}$ Tris- $\mathrm{HCl} \mathrm{pH} 7.2$ (binding buffer). Bacterially expressed AMPK heterotrimers $(\alpha 1 \beta 1 \gamma 1)$ were $\mathrm{Ni}^{2+}$-affinity purified. $4.6 \mathrm{mg}$ of the eluate was subsequently rebuffered to binding buffer. The AMPK sample was split into halves and either loaded to control resin or resin with immobilized $\beta$-cyclodextrin ( $500 \mu \mathrm{l}$ bed volume each). The flow through (FT) was collected, and both columns were washed repeatedly with binding buffer (W1-W3) and then eluted twice with binding buffer containing $5 \mathrm{mM} \beta$-cyclodextrin. $10 \mu \mathrm{l}$ of each fraction was subjected to SDS-PAGE and gel was stained by Coomassie Brilliant Blue. (B) Schematic model illustrating the $\beta$-cyclodextrin binding assay protocol. (C) Histagged CBM (CBM $\beta 1$ or CBM $\beta 2)$ and kinase-deficient AMPK $(\alpha 1 \beta 1 \gamma 1-\mathrm{KD}$ or $\alpha 1 \beta 2 \gamma 1-\mathrm{KD})$ were subjected to the $\beta$-cyclodextrin binding assay and visualized by Coomassie Brilliant Blue Stain. (D) Schematic representation of the hexacistronic expression vector containing 
both AMPK (e.g. $\alpha 1 \beta 1 \gamma 1$ ) and its upstream kinase LKB1-M025 $\alpha$-Strad $\alpha$, allowing for "in vivo" activation of AMPK in the bacterial cytosol and subsequent purification of active hexahistidinetagged AMPK [28]. (E) Detection of purified inactive and active heterotrimeric AMPK $(\alpha 1 \beta 1 \gamma 1$ or $\alpha 1 \beta 2 \gamma 1)$ and AMPK complexes lacking the CBM $(\alpha 1 \beta-\Delta \mathrm{CBM} \gamma 1)$ by Coomassie Brilliant Blue Stain. Recombinant active proteins were bacterially expressed by means of hexacistronic plasmids. (F) Immunoblot analysis of inactive and active (pT172) WT $\alpha 1 \beta 1 \gamma 1 / \alpha 1 \beta 2 \gamma 1$ or truncated AMPK complexes $(\alpha 1 \beta 1 \triangle \mathrm{CBM} \gamma 1)$. AMPK binding to $\beta$-cyclodextrin was assessed using the total AMPK $\alpha$ antibody. Activation of AMPK was assessed using the pT172 antibody. Data are representative of three experiments. I, input; S, supernatant/non-bound protein; $\mathrm{P}$, pellet/bound protein, L, Laemmli buffer/precipitated protein.

AMPK activation by upstream kinases is not directly responsible for the loss of binding ability to carbohydrates

To test whether phosphorylation of T172 in AMPK $\alpha$ was sufficient to cause the loss of carbohydrate-binding, a KD mutant of AMPK $\alpha 1 \beta 1 \gamma 1$ was bacterially expressed in the presence or absence of the LKB1-Mo25 $\alpha$-Strad $\alpha$ complex and subsequently purified. In contrast to WT-AMPK, both T172-phosphorylated and non-phosphorylated KD complexes were found in the pellet fraction, indicating that catalytically inactive AMPK did not lose its ability to bind to the $\beta$-cyclodextrin when co-expressed with the LKB1-Mo25 $\alpha$-Strad $\alpha$ complex (Fig. 2A). Thus, phosphorylation of AMPK at T172 by an upstream kinase is insufficient to trigger the loss of carbohydrate-binding, suggesting that AMPK enzyme activity is required.

To confirm this, AMPK $\alpha 1 \beta 1 \gamma 1, \alpha 1 \beta 2 \gamma 1$ and $\alpha 1 \beta 1-\Delta \mathrm{CBM} \gamma 1$ were expressed as inactive kinases (i.e. without co-expression of the LKB1-Mo25-Strad $\alpha$ complex) for subsequent activation in-vitro before or during the $\beta$-cyclodextrin binding assay. Bacterial expression of recombinant CaMKK2 yields higher purity than LKB1 complex and was therefore used as an alternative upstream kinase of AMPK for these assays. As shown by the phosphorylation of T172, all AMPK isoforms were activated and, as expected, mostly lost their binding ability to the resin material (Fig. 2B), indicating that activation of AMPK leads to loss of its carbohydrate-binding ability irrespective of the identity of the upstream kinase used, CaMKK2 or LKB1. However, when inactive AMPK was initially allowed to bind to the $\beta$-cyclodextrin resin and was subsequently incubated with CaMKK2, both AMPK isoforms retained their ability to bind to the resin, as the proteins were predominantly found in the pellet fraction, despite phosphorylation at T172 (Fig. 2C). The $\beta 2$-isoform showed stronger retention upon activation compared to the $\beta 1$ complex, which corresponds to a higher binding affinity of the $\beta 2$-CBM for $\beta$-cyclodextrin [16]. In this experimental setup the $\alpha 1 \beta 1-\Delta \mathrm{CBM} \gamma 1$ only appears in the input and not in the $\mathrm{S}$ or $\mathrm{P}$ fraction due to its inability to bind the $\beta$-cyclodextrin resin, and therefore the protein is lost upon removal of the supernatant in step 1. Similar results were obtained using $\alpha 2$-containing ( $\alpha 2 \beta 1 \gamma 1$ and $\alpha 2 \beta 2 \gamma 1$ ) AMPK complexes 
A

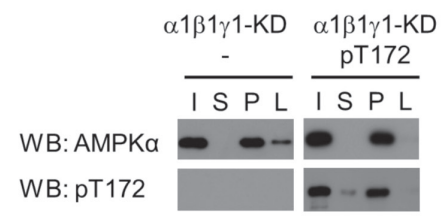

C
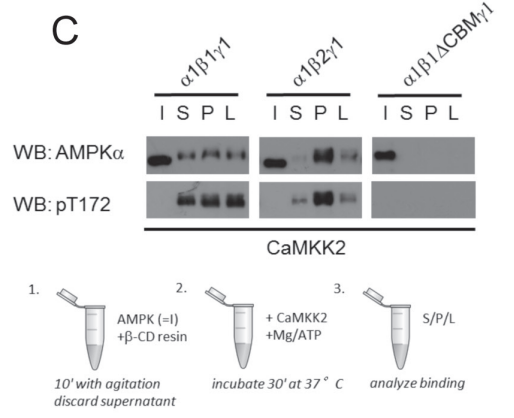

E

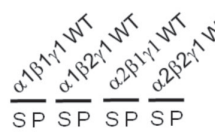

WB: AMPKo

WB: pT172

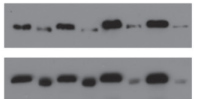

1.
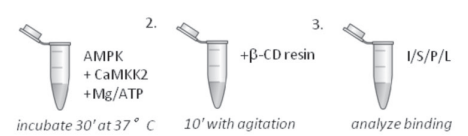

B

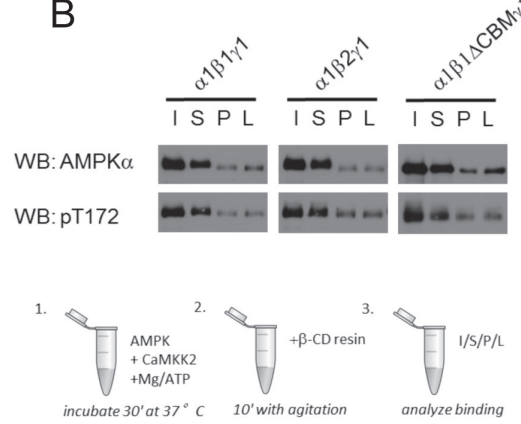

$\mathrm{D}$

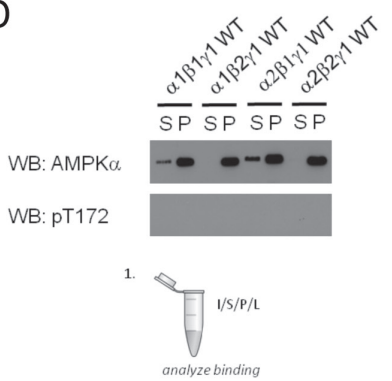

$\mathrm{F}$

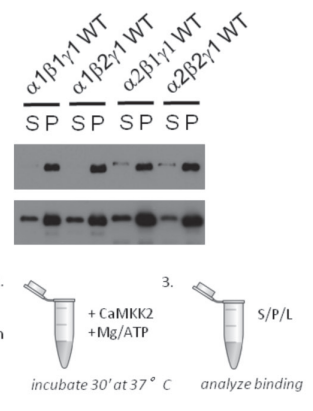

G

\begin{tabular}{|c|c|c|}
\hline -ATP & + ATP & \\
\hline$\alpha 1 \beta 1-\triangle \mathrm{CBM} \gamma 1 \quad \alpha 1 \beta 1 \gamma 1$ & $\alpha 1 \beta 2 \gamma 1 \alpha 1 \beta 1-\triangle \mathrm{CBM} \gamma 1 \alpha 1 \beta 1 \gamma 1$ & $\alpha 1 \beta 2 \gamma 1$ \\
\hline
\end{tabular}

WB: AMPKa

WB: pT172

WB: pT172
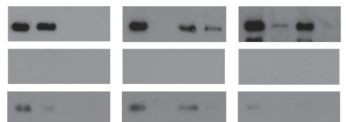

en-
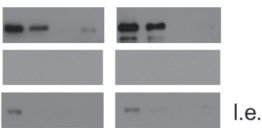

1:100 (active/inactive)

Figure 2. AMPK activation by upstream kinases is not directly responsible for the loss of binding ability to carbohydrates.

(A) Immunoblot of non-phosphorylated and phosphorylated (pT172) recombinant kinasedeficient AMPK ( $\alpha 1 \beta 1 \gamma 1-\mathrm{KD}$ ) (as in Fig. 1) subjected to the $\beta$-cyclodextrin binding assay. (B, C) Immunoblot of WT $(\alpha 1 \beta 1 \gamma 1$ or $\alpha 1 \beta 2 \gamma 1)$ or truncated recombinant AMPK complexes $(\alpha 1 \beta 1 \Delta \mathrm{CBM} \gamma 1$ ) (as in Fig. 1) phosphorylated in vitro by the upstream kinase CaMKK2 (B) prior to exposure to $\beta$-cyclodextrin, or (C) after initial binding to $\beta$-cyclodextrin. (D-F) Four 
different isoforms of AMPK heterotrimers were subjected to the $\beta$-cyclodextrin binding assay. Immunoblot analysis of (D) inactive AMPK complexes, (E) AMPK complexes that were in-vitro phosphorylated by the upstream kinase CaMKK2 prior to $\beta$-cyclodextrin exposure, or (F) AMPK complexes that were phosphorylated in vitro after initial binding to $\beta$-cyclodextrin.

(G) Inactive and active AMPK complexes were mixed (ratio 100:1) and incubated in the presence or absence of ATP and subjected to the $\beta$-cyclodextrin binding assay. In all experiments, the effect on AMPK binding to the model carbohydrate was evaluated. AMPK binding and activation status of AMPK was assessed using the total AMPK $\alpha$ and pT172 antibodies, respectively. Data are representative of three experiments. l.e., long exposure; $\beta$-CD, $\beta$-cyclodextrin.

(Fig. 2D-F). The data, together with the retention of carbohydrate-binding for the phosphorylated KD mutant, underline that phosphorylation of AMPK at T172 is compatible with its binding capacity to carbohydrates. Further, the binding of AMPK to $\beta$-cyclodextrin prior to activation is protective against the loss of binding ability.

We speculated that autophosphorylation could be responsible for the loss of binding to carbohydrates, because autophosphorylation is a consequence of the initial activation of kinases. Moreover, autophosphorylation at multiple sites has been demonstrated for AMPK upon activation $[35,36]$. Experimentally it is difficult to initiate autophosphorylation without kinase activation. However, presuming a bimolecular mechanism it can be tested if the phosphorylation of inactive AMPK by its active counterpart would result in the loss of carbohydrate-binding ability. Hence, inactive recombinant AMPK ( $\alpha 1 \beta 1 \gamma 1, \alpha 1 \beta 2 \gamma 1$ or $\alpha 1 \beta 1-\Delta \mathrm{CBM} \gamma 1$, respectively) was incubated with a very low amount of active AMPK (ratio 100:1). In the absence of ATP, both fulllength AMPK proteins retained their ability to bind to the $\beta$-cyclodextrin resin, whereas the heterotrimer lacking the CBM was not capable of binding (Fig. 2G). However, in the presence of ATP, both AMPK isoforms were found in the supernatant fraction, indicating their loss of binding ability to the $\beta$-cyclodextrin resin despite the lack of T172 phosphorylation by upstream kinases. The presence of the small fraction of active AMPK was confirmed by prolonged exposure (Fig. 2G, lower panel). These results show that a catalytic amount of enzymatically active AMPK is sufficient to prevent inactive AMPK from binding to $\beta$-cyclodextrin in the presence of ATP, suggesting a bimolecular autophosphorylation event precludes AMPK from $\beta$-cyclodextrin binding.

\section{Autophosphorylation of AMPK at $\beta$-T148 causes a loss of binding ability to carbohydrate}

Considering that the binding of $\beta$-cyclodextrin prior to AMPK activation is protective from loss of binding, we speculated that the autophosphorylation event takes place directly in the carbohydrate-binding pocket of the CBM. Inspection of the X-ray structure of the $\beta 1$-CBM identified the threonine-148 (T148) residue as centrally 


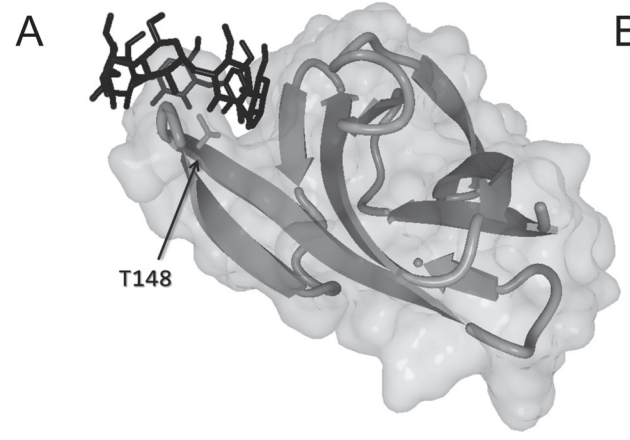

B

C

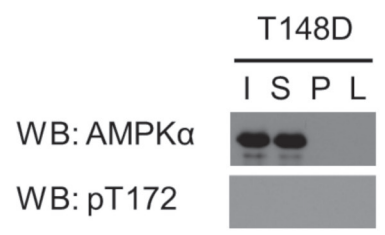

$\mathrm{D}$

WB: AMPKa

WB: pT172
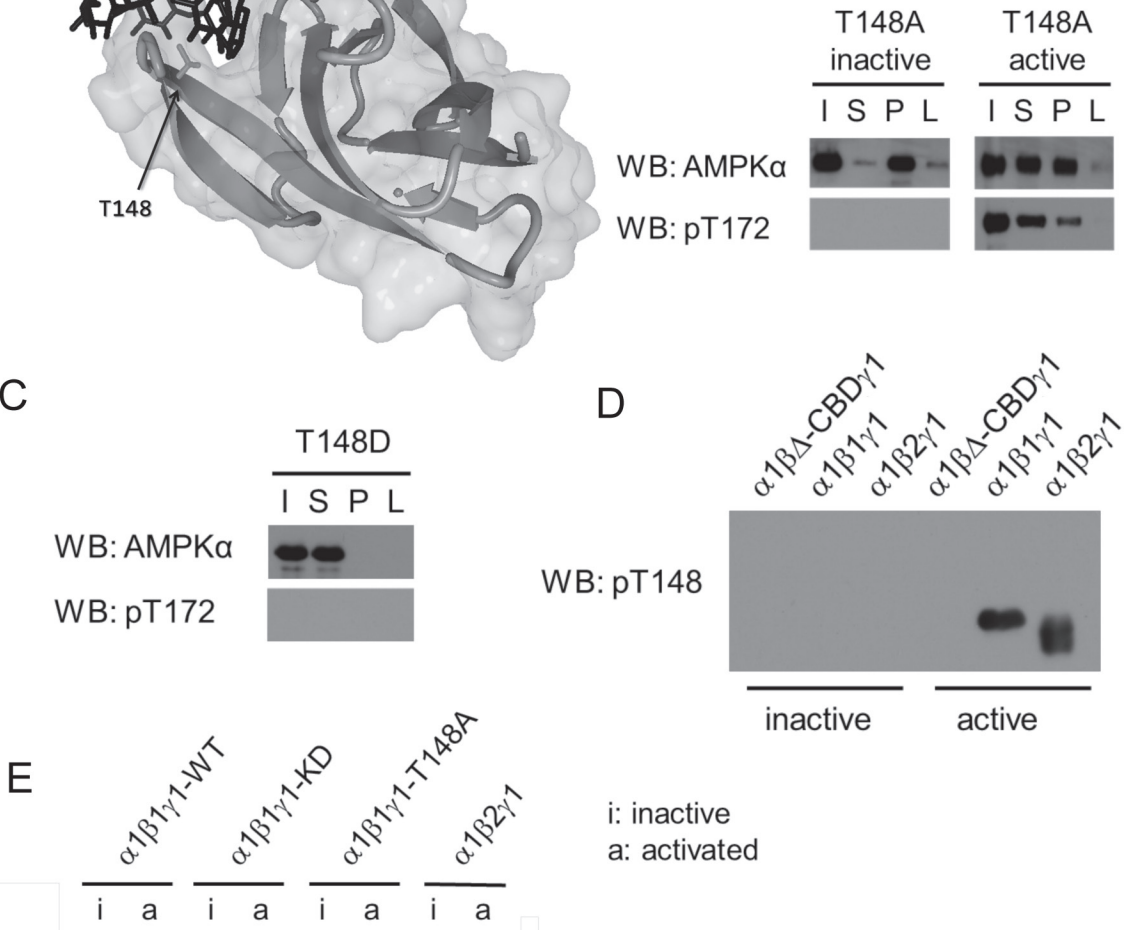

WB: pT148
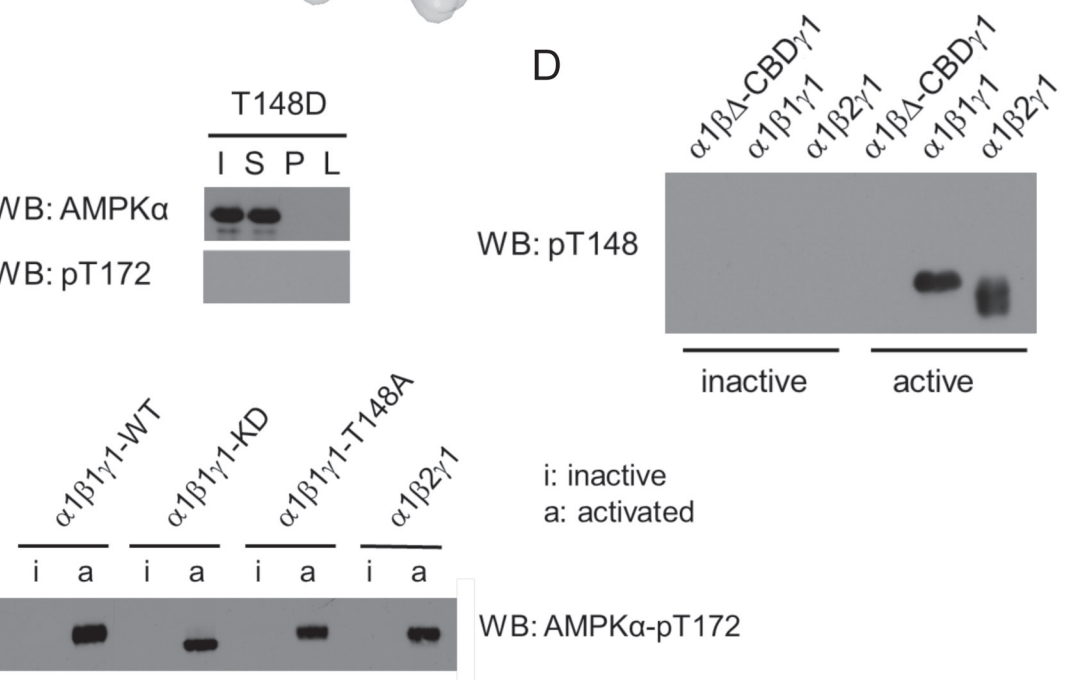

WB: AMPKa-pT172

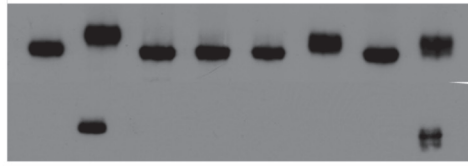

WB: AMPKa

WB: AMPKß-pT148

\section{Figure 3. Autophosphorylation of AMPK at $\beta$-T148 causes a loss of carbohydrate- binding ability.}

(A) Representation of AMPK $\beta 1$-CBM co-crystallized with $\beta$-cyclodextrin (PDB code: 1Z0M). The secondary structure of the protein is represented in light grey ribbon with molecular surface, the $\beta$-cyclodextrin in dark sticks and the side chain of T148 within the carbohydratebinding pocket is highlighted in sticks. This image was generated using the software YASARA View [37]. (B) Binding capacity of inactive and active AMPK bearing a non-phosphorylatable mutation at $\beta 1$-T148 (T148A). (C) Effect of a phospho-mimicking modification of $\beta 1$-T148 (T148D) on AMPK-carbohydrate binding. (D) Determination of T148 phosphorylation on inactive and active recombinant AMPK complexes using a phospho-specific $\beta$-T148 antibody. (E) Immunoblot analysis of inactive and active recombinant AMPK. Wild-type (WT) AMPK or AMPK complexes bearing either a kinase-deficient ( $\alpha 1 \beta 1 \gamma 1-\mathrm{KD}$ ) (as in Fig. 1) or a nonphosphorylatable mutation at residue T148 (T148A) were analyzed for T148 phosphorylation using the phospho-specific T148 antibody. AMPK presence was assessed using the total AMPK $\alpha$ antibody. Activation was determined by the pT172 antibody. Data are representative of three experiments. 
located in the carbohydrate binding pocket (Fig. 3A). Using point mutagenesis, this site was mutated to an alanine (phosphorylation-resistant mutant T148A) in both inactive and active $\alpha 1 \beta 1 \gamma 1$ complexes. In the $\beta$-cyclodextrin binding assay, the inactive mutant conserved its binding capacity (Fig. 3B). However, in contrast to the active WT (Fig. 1F), the active T148A mutant partially conserved its carbohydrate-binding capacity upon co-expression of AMPK with the LKB1-Mo25-Strad $\alpha$ complex (Fig. 3B), implying a protective role of the $\mathrm{T} 148 \mathrm{~A}$ mutation.

To further establish the involvement of T148 phosphorylation in the carbohydratebinding ability of AMPK $\alpha 1 \beta 1 \gamma 1$, a phosphorylation-mimicking T148D mutant was incubated with the $\beta$-cyclodextrin resin. Although not being activated, the T148D mutant was unable to bind to the $\beta$-cyclodextrin resin (Fig. 3C). In addition, a phosphorylation site-specific T148 (pT148) antibody was developed allowing for detection of the modification in AMPK by Western blotting. The recombinant activated WT-AMPK $\alpha 1 \beta 1 \gamma 1$ and $\alpha 1 \beta 2 \gamma 1$ complexes showed a T148 phospho-specific signal, whereas neither the inactive counterparts nor inactive/active $\alpha 1 \beta 1 \Delta \mathrm{CBM} \gamma 1$ showed detectable signals (Fig. 3D). Moreover, the T172 phosphorylated KD and T148A mutants of AMPK $\alpha 1 \beta 1 \gamma 1$ did not show a pT148 signal either (Fig. 3E), thus indicating the specificity of the pT148 antibody. Therefore, the T148 site is indeed autophosphorylated and this modification directly correlates with the inability of AMPK to bind to the $\beta$-cyclodextrin resin. Taken together, these results indicate that autophosphorylation of AMPK at T148 mediates its loss of carbohydrate-binding capacity.

The phosphorylation-mimicking T148D mutation prevents AMPK from binding to glycogen

Next, we investigated whether the T148 phospho-mimicking mutation (T148D) triggers a loss of binding of AMPK to glycogen, its natural ligand, both in the $\beta 1$ - and $\beta 2$-isoforms. Our initial analysis concentrated on the isolated WT and T148D $\beta$-CBMs. Stable cell lines were established that expressed GFP-tagged CBM variants in the human hepatic cell line HepG2 and cultured mouse HL-1 cardiomyocytes. Immunofluorescence showed a marked speckled pattern of the WT GFP-tagged constructs, $\beta 1$-CBM in HepG2 (Fig. 4A) and $\beta 2$-CBM in HL-1 (Fig. 4B) both co-localizing with glycogen. In contrast, the $\beta 1$ - and $\beta 2$-CBM T148D presented a diffuse pattern despite the presence of glycogen, as shown by a glycogen-specific antibody. Similar results were obtained using the Hek293T cell line stably expressing the $\beta 1$-CBM (Fig. 4C): the speckled pattern of the WT-CBM co-localized with glycogen, whereas the diffuse pattern of the mutant was incongruent with the observed glycogen staining. We further assessed the glycogen localization of mCherry-tagged full-length AMPK $\beta 1$-WT 
A

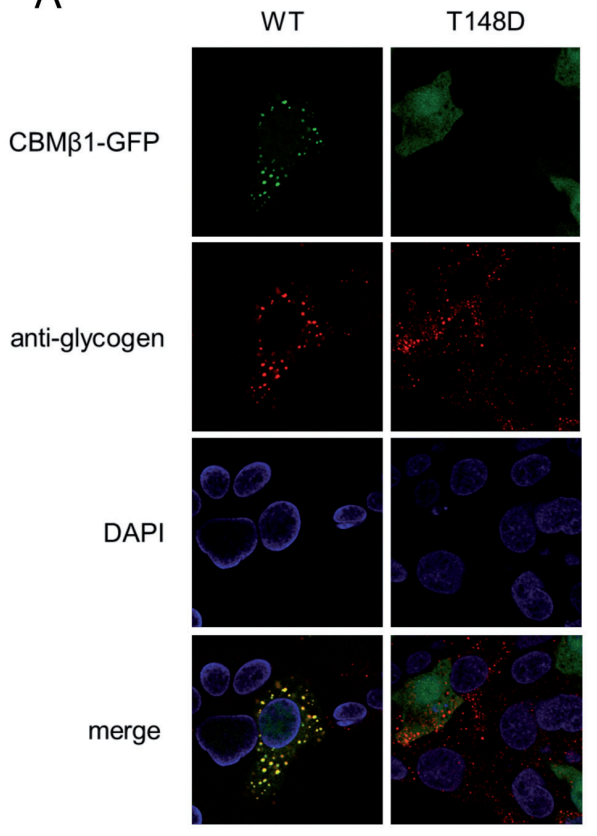

C

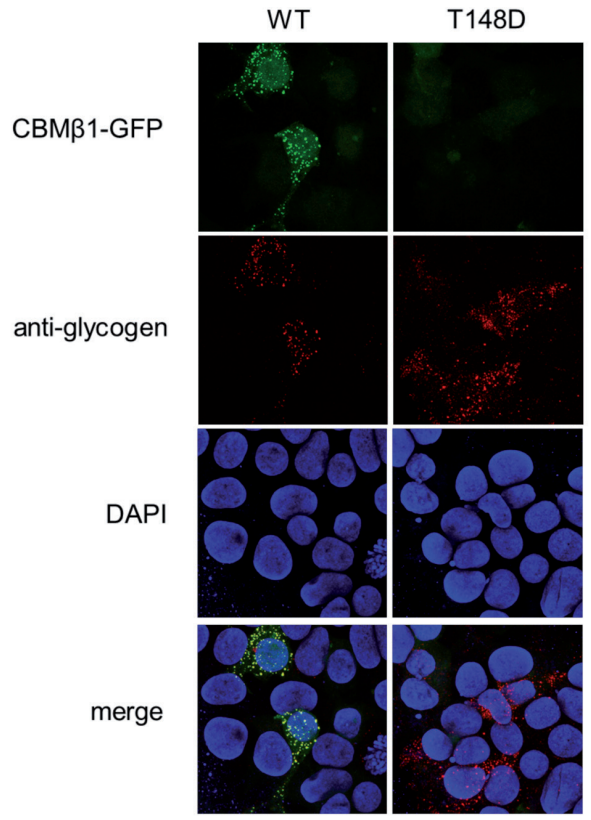

B

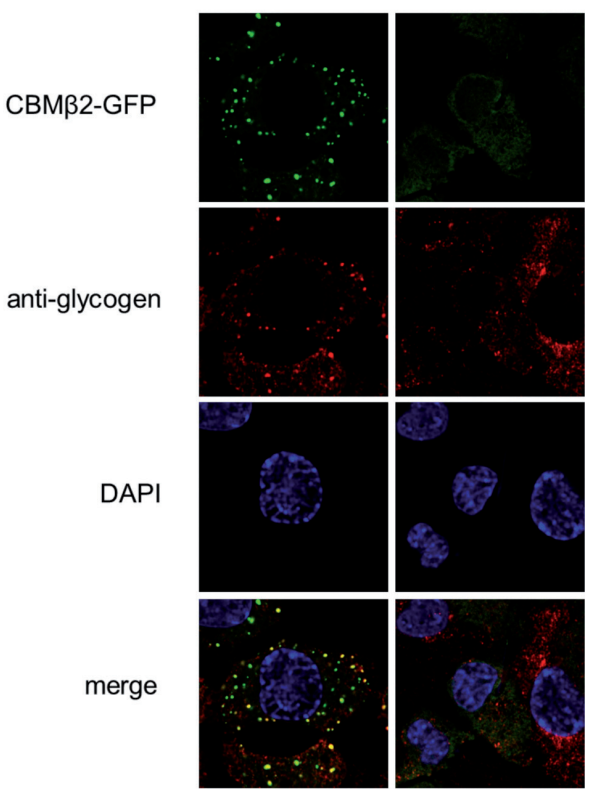

Hek293T

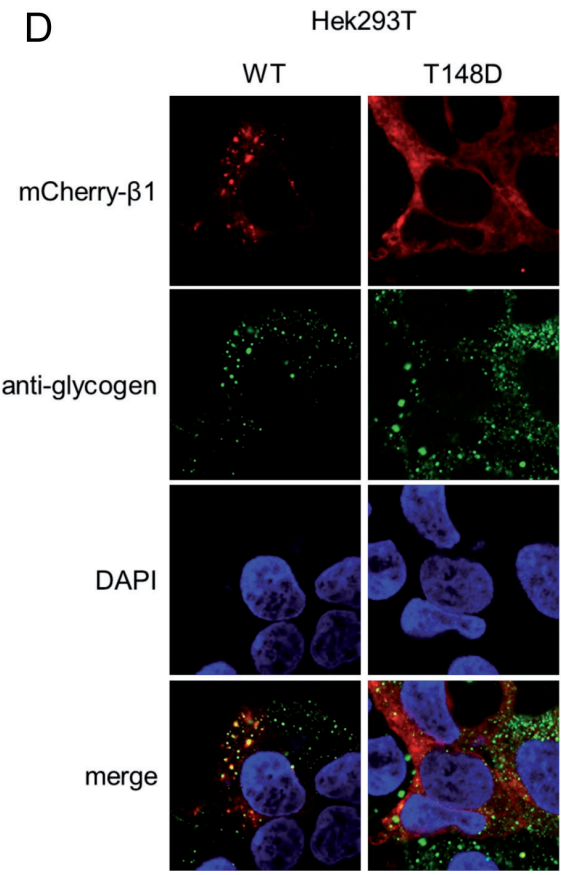

Figure 4. The phosphorylation-mimicking AMPK $\beta$-T148D mutation prevents AMPK from binding to cellular glycogen.

(A) HepG2, (B) HL-1, (C) Hek293T cells stably overexpressing the WT and T148D mutant GFP-tagged CBM $\beta 1$ or CBM $\beta 2$ isoforms (in green) as indicated. Cells were fixed and stained 
with an anti-glycogen antibody that was detected by an Alexa647-labeled secondary antibody (in red) and with DAPI for nuclei (in blue), after which co-localization was assessed by confocal imaging. (D) Co-localization of WT or T148D mCherry-tagged $\beta 1$-AMPK complexes (in red) was assessed as in A and B, but using Alexa488-labeled secondary antibody for glycogen detection (in green). Data are representative of three to five experiments. Data have been adjusted for brightness and contrast to obtain best quality fluorescent images.

and T148D mutant after transient transfection of AMPK subunits in Hek293T cells. Results shown in Figure 4D again revealed co-localization with glycogen for the WT, while the T148D mutant was diffusely located in the cytosol. Altogether, these findings indicate that the CBM of AMPK, expressed as an isolated domain or as full-length protein forming part of the AMPK heterotrimer, naturally binds to glycogen, whereas the phospho-mimicking T148D mutation prevents this binding from occurring.

\section{Endogenous AMPK $\beta$-T148 phosphorylation is a dynamic and regulated process}

In order to investigate the occurrence of T148 phosphorylation in cells and its detection with the pT148 antibody, we immuno-precipitated endogenous AMPK from treated and untreated Hek293T or HepG2 cells (Fig. 5A and B). Importantly, phosphorylation of T148 could be detected in both cell lines, although under different conditions. In Hek293T, only the treatment with AICAR plus insulin led to the detection of the T148 phosphorylation (Fig. 5A) and this treatment also showed the highest level of T172 phosphorylation. In HepG2, however, T148 phosphorylation occurred independently of AMPK activation changes (Fig. 5B). Hence, the data verifies T148 phosphorylation as an endogenous posttranslational modification. In addition, the differences of T148 phosphorylation between cell types and conditions underline the control and dynamics of this post-translational modification.

\section{Glucose deprivation triggers AMPK $\beta$-T148 phosphorylation}

To investigate the signals leading to AMPK T148 autophosphorylation, we tested whether glycogen depletion induced AMPK $\beta$-T148 phosphorylation (Fig. 6A) and compared $\beta 1$-WT with $\beta 1$-T148D mutant AMPK expressing cells (Fig. 6B). Hek293T cells were either triple-transfected (as before) with AMPK $\gamma 1$, myc-tagged AMPK $\alpha 1$ and mCherry-tagged AMPK $\beta 1$ (WT or T148D) or left untransfected, and then treated for 16 hours with a high glucose medium with or without forskolin, a drug which promotes glycogen degradation [38], or with a medium without glucose. As expected, in forskolin-treated or glucose deprived cells, the glycogen content was diminished if compared to control condition in high glucose medium, although statistical significance was only reached in untransfected cells (Fig. 6B). In all of the three culturing conditions the glycogen content was similar in untransfected, $\beta 1-W T$ and $\beta 1-T 148 D$ mutant 


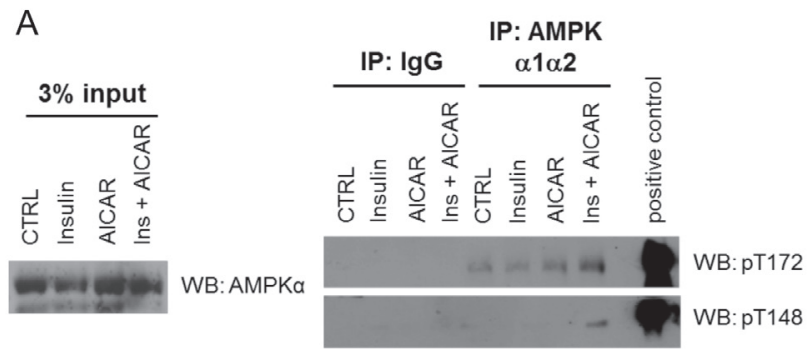

B

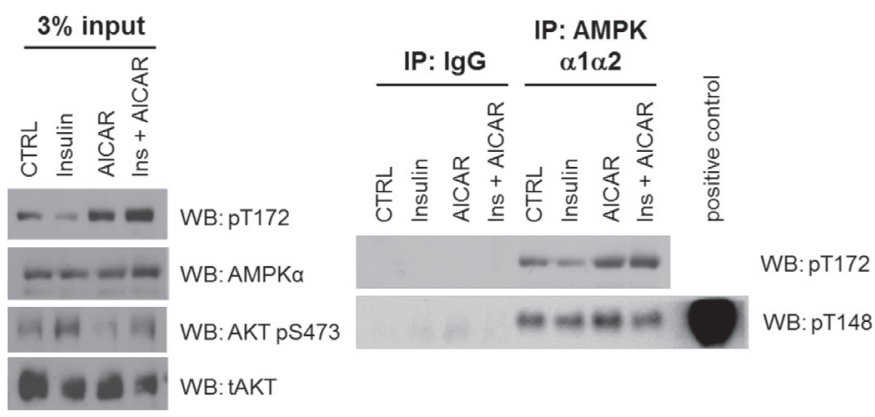

Figure 5. Endogenous AMPK $\beta$-T148 phosphorylation is a dynamic and regulated process.

Immunoprecipitation of endogenous AMPK with AMPK $\alpha 1 / \alpha 2$ antibodies from (A) Hek293T or (B) HepG2 cells. Immunoblot analyses using total AMPK $\alpha$, AMPK $\alpha$-pT172, AMPK $\beta$-pT148, total Akt and Akt-pS473 antibodies. Phosphorylation of AMPK $\beta$-T148 was detected following immunoprecipitation upon single treatment of insulin (100 nM, $15 \mathrm{~min}$ ) or AICAR (1 mM, 45 min), or a combined treatment (Ins + AICAR). All immunocomplexes were assessed for activation of AMPK and $\beta$-T148 phosphorylation using the pT172 and pT148 antibody, respectively. Data are representative of two experiments.

expressing cells. Notably, T148 phosphorylation was specifically induced by glucose deprivation but not forskolin, although both treatments depleted from glycogen (Fig. 6A). The pT148 signal was absent in immuno-precipitates of T148D cells, reassuring that the antibody specifically recognizes the phosphorylation modification on the T148 residue. Given the fact that increased AMPK activation by glucose deprivation is correlated with increased phosphorylation of T148, these data support the cellular AMPK-mediated T148 autophosphorylation mechanism.

\section{$\beta$-T148 phosphorylation plays a role in regulation of glycogen metabolism}

To further explore the physiological significance of T148 phosphorylation we treated cells with different AMPK activators and measured the resulting glycogen content. To allow for comparison of $\beta 1$-WT with $\beta 1$-T148D mutant AMPK, we again employed the Hek293T triple-transfection model. As expected, in serum-starved cells the treatment with oligomycin, A769662 and phenformin resulted in activation of AMPK in $\beta 1$-WT as 
A

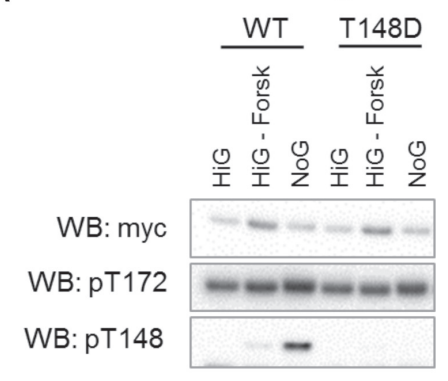

B

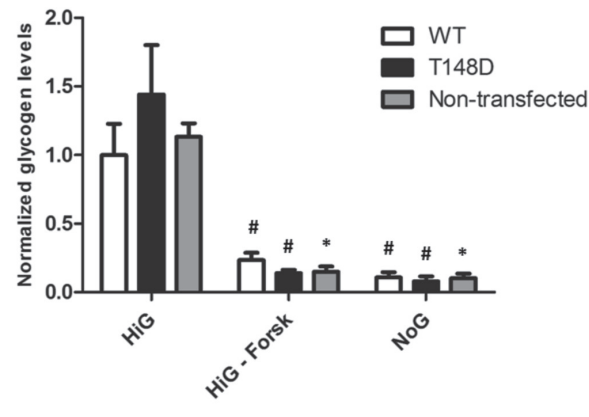

Figure 6. Glucose deprivation triggers AMPK $\beta$-T148 phosphorylation.

(A, B) Hek293T cells transiently overexpressing myc-tagged $\alpha 1, \gamma 1$ and WT or T148D mCherry-tagged $\beta 1$, were either treated for 16 hours with high glucose medium containing serum (HiG), with the same medium supplemented with forskolin $(100 \mu \mathrm{M}$; HiG-Forsk) or with medium containing serum but no glucose (NoG). (A) AMPK $\alpha$ was immunoprecipitated using the myc-tag antibody. Immunoblots using myc-tag antibody following precipitation shows the immunoprecipitation efficiency. Immunoblot with AMPK $\alpha$-pT172 and AMPK $\beta$ pT148 show AMPK activity and autophosphorylation status. (B) Glycogen was quantified biochemically, corrected for protein concentration and normalized to the level of WT cells under HiG. Statistical test: Student's $t$-test HiG vs. treatment within one cell type $(*: p<0.05$, $\#: \mathrm{p}=0.06) \cdot(\mathrm{n}=2)$

well as in $\beta 1$-T148D cells, as observed by an increase in phosphorylation of T172 (Fig. 7A). Further, the pT172 levels were paralleled by similar increases in T148 phosphorylation with the pT148 signal virtually absent in high-glucose treated cells and highest upon phenformin treatment. Cellular glycogen content generally decreased upon AMPK activation in accordance with the expected AMPK-induced shift towards catabolism (Fig. 7C). Statistically significant reductions were observed between vehicle and AICAR or oligomycin-treated WT-transfected cells. Interestingly, in A769662 or AICAR-treated cells, glycogen content was significantly higher in T148D vs. WTtransfected cells, which may relate to AMPK $\beta 1$-T148D being absent from glycogen and thus unable to inhibit glycogen synthesis. Indeed, the AMPK-dependent inhibition of glycogen synthase (GS) by phosphorylation at serine 7 (pS7) was strongly increased in WT-transfected cells upon oligomycin and A769662, whereas the respective pS7 signals were fainter in the T148D-transfected cells (Fig. 7B). Moreover, the glycogen content of WT-transfected cells treated with phenformin showed neither a difference with the vehicle-treated WT cells, nor with the phenformin-treated T148D cells (Fig 7C). Interestingly, this absence of difference correlated with the highest T148 phosphorylation (Fig. 7A), suggesting that the autophosphorylation was sufficient to mimic the effect of the mutation. Taken together, T148 is phosphorylated upon AMPK activation and thus prevents AMPK from binding to glycogen, which affects glycogen metabolism. 
A

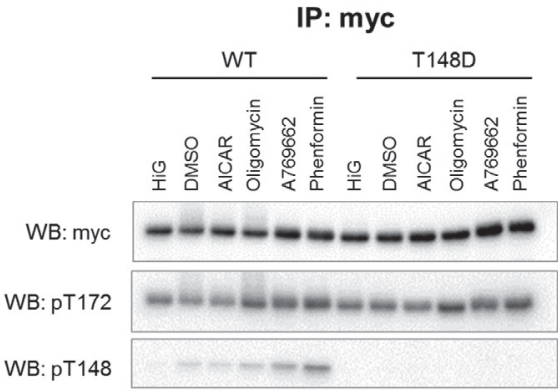

B

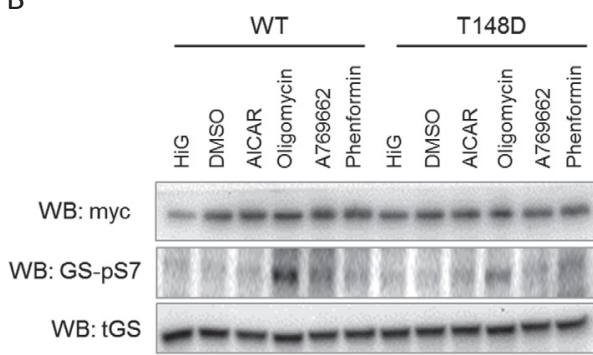

C

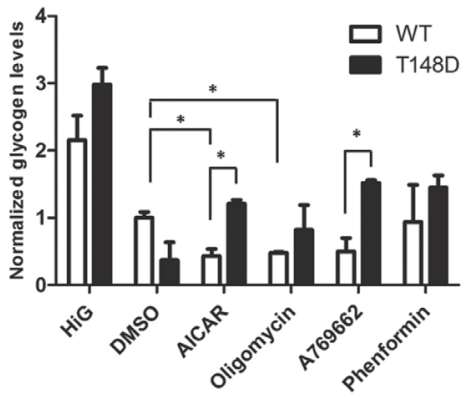

Figure 7. AMPK $\beta$-T148 phosphorylation plays a role in regulation of glycogen metabolism.

(A-C) Hek293T cells transiently overexpressing myc-tagged $\alpha 1, \gamma 1$, and $\beta 1$-mCherry, either wild-type (WT) or $\beta 1$ carrying the phospho-mimicking T148D (T148D) were serum-starved for $16 \mathrm{~h}$ prior to treatment with either high glucose medium (HiG) or DMSO, AICAR (1.5 mM), Oligomycin (3 uM), A769662 (100 uM) or Phenformin (1.5 mM) for 1 hour. (A) AMPKa was immunoprecipitated using the myc-tag antibody. Immunoblot using myc-tag antibody following precipitation shows the immunoprecipitation efficiency. Immunoblots with AMPK $\alpha$-pT172 and AMPK $\beta$-pT148 show AMPK activity and autophosphorylation status. (B) Immunoblots of the associated input samples show the expression levels of overexpressed myc-protein and of glycogen synthase (tGS) and the phosphorylation status of glycogen synthase (GS-pS7). (C) Glycogen was quantified biochemically, corrected for protein concentration and normalized to the level of WT cells under HiG. Statistical test: Student's t-test $(*: \mathrm{p}<0.05)$

\section{Discussion}

In this study, we investigated the molecular mechanism causing AMPK to refrain from binding to glycogen thereby affecting glycogen turnover. Our results demonstrate that bimolecular AMPK autophosphorylation at $\beta$-subunit T148 interferes with its carbohydrate-binding capacity. The T148 site is located within the center of the carbohydrate-binding pocket, thereby controlling the attachment to glycogen. Accordingly, autophosphorylation at T148 is prevented by carbohydrate occupancy. 
We also provide evidence that WT isolated CBMs and full-length $\beta 1$-subunit as part of the AMPK heterotrimer localizes to glycogen particles, whereas the respective T148D mutants do not bind cellular glycogen. In addition, we show that activation of AMPK enhances cellular T148 phosphorylation and provide first evidence for involvement of $\beta 1$-T148 phosphorylation in preventing AMPK from glycogen metabolism regulation.

It is established that many protein kinases catalyze their own activation by autophosphorylation [39]. Autophosphorylation of AMPK has been described previously [11, 35, 36] but, at present, little is known about the biological functions of AMPK autophosphorylation. Autophosphorylation of S108 is required for activation of AMPK by the small molecule A-769662 independently of $\alpha$ T172 phosphorylation [40], but the physiological function of this modification has remained elusive so far. The phosphorylation of S24/25 in the $\beta 1$ subunit leads to nuclear exclusion [20] and S24/25 were identified as autophosphorylation sites [35, 36]. Here, we identify T148 as a new autophosphorylation site and also ascribe its function to regulation of subcellular localization. Likewise, Feng and Hannun [41] reported a dissociation of protein kinase $\mathrm{C}$ (PKC) from the plasma membrane to the cytosol upon autophosphorylation. Also, the kinase ERK1/2 translocates to the nucleus upon autophosphorylation induced by extracellular signal, where it phosphorylates nuclear targets that are known to initiate cardiac hypertrophy [42]. Hence, autophosphorylation, which more commonly has been regarded as a 'mistake' of active kinases, rather emerges as a self-regulatory mechanism with possible consequences in subcellular targeting, and thus relevant for health and disease.

Our investigation revealed that little amounts of active AMPK were sufficient to prevent catalytically inactive AMPK from binding to glycogen, suggesting that a bimolecular autophosphorylation event is involved in this process. Therefore, it seems possible that few activated AMPK molecules are able to prevent glycogen binding of the entire pool of AMPK molecules, which would explain why the correlation of T172 and T148 phosphorylation was not always seen in cells. However, we can expect that the cell will have preventive measures, such as dephosphorylation of T148 in order to restore glycogen binding. The phosphatase responsible for dephosphorylation therefore awaits identification.

Although some proteins can indirectly associate to glycogen via interacting with other glycogen-binding proteins [43], most proteins capable of directly attaching to glycogen or other polysaccharides possess a CBM. Regulation of glycogen binding by post-translational modifications has been previously reported. Notably, the protein phosphatase 1 (PP1) was found to detach from glycogen upon phosphorylation of its CBM-containing regulatory subunit G [44]. Later work showed that this phosphorylation led to dissociation of the PP1 from the G-subunit and its translocation to cytosol, while 
the G-subunit stayed bound to glycogen [45]. Glycogen synthase, which does not carry a CBM, has also been reported to change cellular localization upon phosphorylation: when phosphorylated at site $1 \mathrm{~b}$, it associates with intramyofibrillar glycogen particles, whereas the site 2+2a phosphorylated enzyme binds to intermyofibrillar particles [46]. Nevertheless, both of these phosphorylation events are unrelated to the glycogenbinding site of the glycogen synthase [18]. AMPK carries a $\beta$-subunit CBM that is known to target proteins to glycogen $[5,6]$, but regulation of its glycogen-binding function is unknown as AMPK is found in various subcellular compartments including those devoid of glycogen. In this current study, we provide evidence for a direct modification within the CBM, which we believe is the first example of such modification within a CBM resulting in loss of carbohydrate-binding affinity. We report the blocking of AMPK glycogen binding by T148 autophosphorylation. Indeed, X-ray structural data indicated that T148 of the AMPK- $\beta$ subunit is positioned in the center of the carbohydratebinding pocket and thus could be predicted to cause loss of binding upon phosphorylation. In support, our results revealed that the phosphorylation mimicking AMPK-T148D mutant was indeed incapable of binding to carbohydrates, suggesting that T148 phosphorylation plays a role in localizing AMPK away from carbohydrates. This novel mechanism of AMPK regulation is furthermore consistent with studies using large-scale phospho-proteome analyses and reporting T148 as a $\beta 2$ phosphorylation site $[47,48]$. Notably, in our study we find the $\beta 1$ and $\beta 2$-subunits are both modified by autophosphorylation at the T148 site. Although the antibody was raised against the $\beta 2$-sequence, it recognizes the modification in both isoforms (Fig. 3E). Therefore, based on our data we cannot draw conclusions on isoform-specific differences of cellular T148 phosphorylation.

To further evaluate the significance of T148, we addressed the question whether $\beta$-T148 is being phosphorylated in cellular models. Our data showed that the levels of T148 phosphorylation were mostly correlated with AMPK activity. In fact, based on our findings, we can assume that the molecular T148 autophosphorylation mechanism we describe here and binding to glycogen occurs in a mutually exclusive manner. In other words, AMPK- carbohydrate binding results in masking of the T148 residue, which subsequently prevents AMPK from autophosphorylation. In line with this, the binding of $\beta$-cyclodextrin prior to activation was protective for the loss of carbohydrate binding ability. Moreover, insulin treatment prevented further increases of AICAR-mediated T148 phosphorylation in HepG2 (Fig. 5B). This observation could be related to the fact that insulin promotes glycogen synthesis [49], thereby stimulating AMPK binding to glycogen and preventing it from autophosphorylation at T148. As a possible interpretation of our results, even if AMPK in response to stresses would get very highly activated, T148-phosphorylated and, thus, prevented from interacting with 
glycogen, the glycogen-residing part of the AMPK pool would remain bound to carry on with AMPK activities at glycogen.

The precise roles of AMPK associated with glycogen are still subject to debate. On the one hand, AMPK stimulates GLUT4-mediated myocellular glucose uptake [50,51] and co-immunoprecipitates with glycogen-binding proteins, such as glycogen phosphorylase [52], glycogen debranching enzyme [53] and glycogen synthase [54], suggesting AMPK plays a role in the disposal of glucose into glycogen. In support of this notion, the phosphorylation of glycogen synthase by AMPK switches off the synthesis of glycogen $[17,55]$ and that of laforin reduces phosphatase activity and regulates its interaction with malin, thereby playing a major role in glycogen metabolism [56]. However, on the other hand, Hunter et al. [54] delimited the supposed role of AMPK on glycogen synthesis by showing that the AMPK-mediated inactivation of glycogen synthase can be overridden by increased glucose-6-phosphate levels. Partly in line with this, McBride et al. [57] showed a clear inhibition of AMPK when bound to certain branch points of glycogen, raising a doubt on the ability of AMPK to quickly phosphorylate its glycogen-bound downstream targets. Recently, Li et al. showed that binding of the CBM to carbohydrates destabilizes the CBM-kinase domain interaction thereby increasing the accessibility of the kinase domain for T172 dephosphorylation [58], which is consistent with an inhibitory effect of glycogen-binding on AMPK kinase activity. Our findings suggest irrelevance of AMPK glycogen-localization for the process of glycogen degradation (Fig. 6). In contrast, the increased glycogen content in $\beta 1$ T148D mutant vs. $\beta 1$-WT expressing cells upon AMPK activation (Fig. 7C) could be interpreted as augmented rate of glycogenesis due to loss of AMPK glycogen localization and thus exclusion from glycogen synthesis inhibition. In any case, the unravelling of a new mechanism allowing for AMPK to stay unbound in the cytosol has consequences for our understanding of the kinase function, especially in the myocellular cell types where glycogen is a major fuel resource [59]. Hence, future efforts will be focused on determining the biological roles of $\beta$-T148 phosphorylation in cardiac or skeletal muscle.

In summary, using cell-free systems and cellular models, we shed light on a novel molecular mechanism that is responsible for the loss of AMPK-glycogen interaction. T148 autophosphorylation blocks the carbohydrate-binding pocket of the $\beta$-CBM, thus adding a new layer of complexity and identifying an unexpected switch regulating AMPK subcellular localization with relevance for glycogen metabolism. 


\section{References}

1. Janecek, S., Svensson, B. and MacGregor, E.A., Structural and evolutionary aspects of two families of non-catalytic domains present in starch and glycogen binding proteins from microbes, plants and animals. Enzyme Microb Technol, 2011. 49(5): p. 429-440.

2. Cantarel, B.L., Coutinho, P.M., Rancurel, C., Bernard, T., Lombard, V. and Henrissat, B., The Carbohydrate-Active EnZymes database (CAZy): an expert resource for Glycogenomics. Nucleic acids research, 2009. 37(Database issue): p. D233238.

3. Shoseyov, O., Shani, Z. and Levy, I., Carbohydrate binding modules: biochemical properties and novel applications. Microbiol Mol Biol Rev, 2006. 70(2): p. 283-295.

4. Guillen, D., Sanchez, S. and Rodriguez-Sanoja, R., Carbohydrate-binding domains: multiplicity of biological roles. Appl Microbiol Biotechnol, 2010. 85(5): p. 1241-1249.

5. Polekhina, G., Gupta, A., Michell, B.J., van Denderen, B., Murthy, S., Feil, S.C., Jennings, I.G., Campbell, D.J., Witters, L.A., Parker, M.W., Kemp, B.E. and Stapleton, D., AMPK beta subunit targets metabolic stress sensing to glycogen. Current biology : CB, 2003. 13(10): p. 867-871.

6. Hudson, E.R., Pan, D.A., James, J., Lucocq, J.M., Hawley, S.A., Green, K.A., Baba, O., Terashima, T. and Hardie, D.G., A novel domain in AMP-activated protein kinase causes glycogen storage bodies similar to those seen in hereditary cardiac arrhythmias. Current biology : CB, 2003. 13(10): p. 861-866.

7. Wiatrowski, H.A., Van Denderen, B.J., Berkey, C.D., Kemp, B.E., Stapleton, D. and Carlson, M., Mutations in the gal83 glycogen-binding domain activate the snf1/gal83 kinase pathway by a glycogen-independent mechanism. Molecular and cellular biology, 2004. 24(1): p. 352-361.

8. Avila-Castaneda, A., Gutierrez-Granados, N., Ruiz-Gayosso, A., Sosa-Peinado, A., MartinezBarajas, E. and Coello, P., Structural and functional basis for starch binding in the SnRK1 subunits AKINbeta2 and AKINbetagamma. Front Plant Sci, 2014. 5: p. 199.

9. Fogarty, S. and Hardie, D.G., Development of protein kinase activators: AMPK as a target in metabolic disorders and cancer. Biochim Biophys Acta, 2010. 1804(3): p. 581-591.

10. Hardie, D.G., Ross, F.A. and Hawley, S.A., AMPK: $a$ nutrient and energy sensor that maintains energy homeostasis. Nat Rev Mol Cell Biol, 2012. 13(4): p. 251-262.

11. Suter, M., Riek, U., Tuerk, R., Schlattner, U., Wallimann, T. and Neumann, D., Dissecting the role of 5'-AMP for allosteric stimulation, activation, and deactivation of AMP-activated protein $\mathrm{ki}$ nase. J Biol Chem, 2006. 281(43): p. 3220732216.
12. Hegarty, B.D., Turner, N., Cooney, G.J. and Kraegen, E.W., Insulin resistance and fuel homeostasis: the role of AMP-activated protein kinase. Acta Physiol (Oxf), 2009. 196(1): p. 129-145.

13. Mihaylova, M.M. and Shaw, R.J., The AMPK signalling pathway coordinates cell growth, autophagy and metabolism. Nat Cell Biol, 2011. 13(9): p. 1016-1023.

14. Sanders, M.J., Grondin, P.O., Hegarty, B.D., Snowden, M.A. and Carling, D., Investigating the mechanism for AMP activation of the AMP-activated protein kinase cascade. Biochem J, 2007. 403(1): p. 139-148.

15. Bieri, M., Mobbs, J.I., Koay, A., Louey, G., Mok, Y.F. Hatters, D.M., Park, J.T., Park, K.H., Neumann, D., Stapleton, D. and Gooley, P.R., AMP-activated protein kinase beta-subunit requires internal motion for optimal carbohydrate binding. Biophys J, 2012. 102(2): p. 305-314

16. Koay, A., Woodcroft, B., Petrie, E.J., Yue, H., Emanuelle, S., Bieri, M., Bailey, M.F., Hargreaves, M., Park, J.T., Park, K.H., Ralph, S., Neumann, D., Stapleton, D. and Gooley, P.R., AMPK beta subunits display isoform specific affinities for carbohydrates. FEBS Lett, 2010. 584(15): p. 3499 3503.

17. Jorgensen, S.B., Nielsen, J.N., Birk, J.B., Olsen, G.S., Viollet, B., Andreelli, F., Schjerling, P., Vaulont, S., Hardie, D.G., Hansen, B.F., Richter, E.A. and Wojtaszewski, J.F., The alpha2-5'AMP-activated protein kinase is a site 2 glycogen synthase kinase in skeletal muscle and is responsive to glucose loading. Diabetes, 2004. 53(12): p. 30743081.

18. Diaz, A., Martinez-Pons, C., Fita, I., Ferrer, J.C. and Guinovart, J.J., Processivity and subcellular localization of glycogen synthase depend on a non-catalytic high affinity glycogen-binding site. J Biol Chem, 2011. 286(21): p. 18505-18514.

19. Abu-Elheiga, L., Brinkley, W.R., Zhong, L., Chirala, S.S., Woldegiorgis, G. and Wakil, S.J., The subcellular localization of acetyl-CoA carboxylase 2. Proceedings of the National Academy of Sciences of the United States of America, 2000. 97(4): p. 1444-1449.

20. Warden, S.M., Richardson, C., O’Donnell, J., Jr., Stapleton, D., Kemp, B.E. and Witters, L.A., Posttranslational modifications of the beta-1 subunit of AMP-activated protein kinase affect enzyme activity and cellular localization. Biochem J, 2001. 354(Pt 2): p. 275-283.

21. Oakhill, J.S., Chen, Z.P., Scott, J.W., Steel, R., Castelli, L.A., Ling, N., Macaulay, S.L. and Kemp, B.E., beta-Subunit myristoylation is the gatekeeper for initiating metabolic stress sensing by AMPactivated protein kinase (AMPK). Proceedings of the National Academy of Sciences of the United States of America, 2010. 107(45): p. $19237-$ 19241. 
22. Kazgan, N., Williams, T., Forsberg, L.J. and Brenman, J.E., Identification of a nuclear export signal in the catalytic subunit of AMP-activated protein kinase. Mol Biol Cell, 2010. 21(19): p. 3433-3442.

23. Kim, N., Lee, J.O., Lee, H.J., Lee, S.K., Moon, J.W., Kim, S.J., Park, S.H. and Kim, H.S., AMPKalpha2 translocates into the nucleus and interacts with hnRNP H: implications in metformin-mediated glucose uptake. Cellular signalling, 2014. 26(9): p. 1800-1806.

24. Neumann, D., Woods, A., Carling, D., Wallimann, T. and Schlattner, U., Mammalian AMP-activated protein kinase: functional, heterotrimeric complexes by co-expression of subunits in Escherichia coli. Protein expression and purification, 2003. 30(2): p. 230-237.

25. Riek, U., Scholz, R., Konarev, P., Rufer, A., Suter, M., Nazabal, A., Ringler, P., Chami, M., Muller, S.A., Neumann, D., Forstner, M., Hennig, M., Zenobi, R., Engel, A., Svergun, D., Schlattner, U. and Wallimann, T., Structural properties of AMP-activated protein kinase: dimerization, molecular shape, and changes upon ligand binding. J Biol Chem, 2008. 283(26): p. 18331-18343.

26. Neumann, D., Suter, M., Tuerk, R., Riek, U. and Wallimann, T., Co-expression of LKB1, MO25alpha and STRADalpha in bacteria yield the functional and active heterotrimeric complex. Molecular biotechnology, 2007. 36(3): p. 220-231.

27. Woods, A., Vertommen, D., Neumann, D., Turk, R., Bayliss, J., Schlattner, U., Wallimann, T., Carling, D. and Rider, M.H., Identification of phosphorylation sites in AMP-activated protein kinase (AMPK) for upstream AMPK kinases and study of their roles by site-directed mutagenesis. J Biol Chem, 2003. 278(31): p. 28434-28442.

28. Bullen, J.W., Balsbaugh, J.L., Chanda, D., Shabanowitz, J., Hunt, D.F., Neumann, D. and Hart, G.W., Cross-talk between two essential nutrientsensitive enzymes: O-GlcNAc transferase (OGT) and AMP-activated protein kinase (AMPK). J Biol Chem, 2014. 289(15): p. 10592-10606.

29. Scholz, R., Sidler, C.L., Thali, R.F., Winssinger, N., Cheung, P.C. and Neumann, D., Autoactivation of transforming growth factor beta-activated kinase 1 is a sequential bimolecular process. J Biol Chem, 2010. 285(33): p. 25753-25766.

30. Scholz, R., Suter, M., Weimann, T., Polge, C., Konarev, P.V., Thali, R.F., Tuerk, R.D., Viollet, B., Wallimann, T., Schlattner, U. and Neumann, D., Homo-oligomerization and activation of AMPactivated protein kinase are mediated by the kinase domain alphaG-helix. J Biol Chem, 2009. 284(40): p. 27425-27437.

31. McMahon, R.J. and Frost, S.C., Glycogen: a carbohydrate source for GLUT-1 glycosylation during glucose deprivation of 3T3-L1 adipocytes. Am J Physiol, 1996. 270(4 Pt 1): p. E640-645.

32. Kinsella, T.M. and Nolan, G.P., Episomal vectors rapidly and stably produce high-titer recombinant retrovirus. Human gene therapy, 1996. 7(12): p. 1405-1413.
33. Morgenstern, J.P. and Land, H., Advanced mammalian gene transfer: high titre retroviral vectors with multiple drug selection markers and a complementary helper-free packaging cell line. Nucleic acids research, 1990. 18(12): p. 35873596.

34. Voncken, J.W., Niessen, H., Neufeld, B., Rennefahrt, U., Dahlmans, V., Kubben, N., Holzer, B., Ludwig, S. and Rapp, U.R., MAPKAP kinase $3 p K$ phosphorylates and regulates chromatin association of the polycomb group protein Bmi1. J Biol Chem, 2005. 280(7): p. 5178-5187.

35. Mitchelhill, K.I., Michell, B.J., House, C.M., Stapleton, D., Dyck, J., Gamble, J., Ullrich, C., Witters, L.A. and Kemp, B.E., Posttranslational modifications of the 5'-AMP-activated protein kinase beta1 subunit. J Biol Chem, 1997. 272(39): p. 24475-24479.

36. Tuerk, R.D., Auchli, Y., Thali, R.F., Scholz, R., Wallimann, T., Brunisholz, R.A. and Neumann, D., Tracking and quantification of 32P-labeled phosphopeptides in liquid chromatography matrix-assisted laser desorption/ionization mass spectrometry. Anal Biochem, 2009. 390(2): p. 141-148.

37. Krieger, E., Koraimann, G. and Vriend, G., Increasing the precision of comparative models with YASARA NOVA--a self-parameterizing force field. Proteins, 2002. 47(3): p. 393-402.

38. Singh, P.K., Singh, S. and Ganesh, S., The laforinmalin complex negatively regulates glycogen synthesis by modulating cellular glucose uptake via glucose transporters. Molecular and cellular biology, 2012. 32(3): p. 652-663.

39. Hashimoto, Y.K., Satoh, T., Okamoto, M. and Takemori, H., Importance of autophosphorylation at Ser186 in the A-loop of salt inducible kinase 1 for its sustained kinase activity. Journal of cellular biochemistry, 2008. 104(5): p. 17241739.

40. Scott, J.W., Ling, N., Issa, S.M., Dite, T.A., O’Brien, M.T., Chen, Z.P., Galic, S., Langendorf, C.G., Steinberg, G.R., Kemp, B.E. and Oakhill, J.S., Small molecule drug A-769662 and AMP synergistically activate naive AMPK independent of upstream kinase signaling. Chem Biol, 2014. 21(5): p. 619-627.

41. Feng, X. and Hannun, Y.A., An essential role for autophosphorylation in the dissociation of activated protein kinase $C$ from the plasma membrane. J Biol Chem, 1998. 273(41): p. 2687026874.

42. Lorenz, K., Schmitt, J.P., Schmitteckert, E.M. and Lohse, M.J., A new type of ERK1/2 autophosphorylation causes cardiac hypertrophy. Nat Med, 2009. 15(1): p. 75-83.

43. Vernia, S., Solaz-Fuster, M.C., Gimeno-Alcaniz, J.V., Rubio, T., Garcia-Haro, L., Foretz, M., de Cordoba, S.R. and Sanz, P., AMP-activated protein kinase phosphorylates $R 5 / P T G$, the glycogen targeting subunit of the R5/PTG-protein phosphatase 1 holoenzyme, and accelerates its downregulation by the laforin-malin complex. J Biol Chem, 2009. 284(13): p. 8247-8255. 
44. Hiraga, A. and Cohen, P., Phosphorylation of the glycogen-binding subunit of protein phosphatase-1G by cyclic-AMP-dependent protein kinase promotes translocation of the phosphatase from glycogen to cytosol in rabbit skeletal muscle. European journal of biochemistry / FEBS, 1986. 161(3): p. 763-769.

45. MacKintosh, C., Campbell, D.G., Hiraga, A. and Cohen, P., Phosphorylation of the glycogen-binding subunit of protein phosphatase-1G in response to adrenalin. FEBS Lett, 1988. 234(1): p. 189-194.

46. Prats, C., Gomez-Cabello, A. and Hansen, A.V., Intracellular compartmentalization of skeletal muscle glycogen metabolism and insulin signalling. Exp Physiol, 2011. 96(4): p. 385-390.

47. Han, G., Ye, M., Liu, H., Song, C., Sun, D., Wu, Y., Jiang, X., Chen, R., Wang, C., Wang, L. and Zou, H., Phosphoproteome analysis of human liver tissue by long-gradient nanoflow LC coupled with multiple stage MS analysis. Electrophoresis, 2010. 31(6): p. 1080-1089.

48. Bian, Y., Song, C., Cheng, K., Dong, M., Wang, F., Huang, J., Sun, D., Wang, L., Ye, M. and Zou, H., An enzyme assisted RP-RPLC approach for in-depth analysis of human liver phosphoproteome. Journal of proteomics, 2014. 96: p. 253-262.

49. Bouskila, M., Hirshman, M.F., Jensen, J., Goodyear, L.J. and Sakamoto, K., Insulin promotes glycogen synthesis in the absence of GSK3 phosphorylation in skeletal muscle. Am J Physiol Endocrinol Metab, 2008. 294(1): p. E28-35.

50. Hayashi, T., Hirshman, M.F., Kurth, E.J., Winder, W.W. and Goodyear, L.J., Evidence for 5' AMP-activated protein kinase mediation of the effect of muscle contraction on glucose transport. Diabetes, 1998. 47(8): p. 1369-1373.

51. Kurth-Kraczek, E.J., Hirshman, M.F., Goodyear, L.J. and Winder, W.W., 5' AMP-activated protein kinase activation causes GLUT4 translocation in skeletal muscle. Diabetes, 1999. 48(8): p. 16671671.

52. Chen, Z., Heierhorst, J., Mann, R.J., Mitchelhill, K.I., Michell, B.J., Witters, L.A., Lynch, G.S., Kemp, B.E. and Stapleton, D., Expression of the AMPactivated protein kinase beta1 and beta2 subunits in skeletal muscle. FEBS Lett, 1999. 460(2): p. 343-348.
53. Sakoda, H., Fujishiro, M., Fujio, J., Shojima, N., Ogihara, T., Kushiyama, A., Fukushima, Y., Anai, M., Ono, H., Kikuchi, M., Horike, N., Viana, A.Y., Uchijima, Y., Kurihara, H. and Asano, T., Glycogen debranching enzyme association with betasubunit regulates AMP-activated protein kinase activity. Am J Physiol Endocrinol Metab, 2005. 289(3): p. E474-481.

54. Hunter, R.W., Treebak, J.T., Wojtaszewski, J.F. and Sakamoto, K., Molecular mechanism by which AMP-activated protein kinase activation promotes glycogen accumulation in muscle. Diabetes, 2011. 60(3): p. 766-774

55. Carling, D. and Hardie, D.G., The substrate and sequence specificity of the AMP-activated protein kinase. Phosphorylation of glycogen synthase and phosphorylase kinase. Biochim Biophys Acta, 1989. 1012(1): p. 81-86.

56. Roma-Mateo, C., Solaz-Fuster Mdel, C., GimenoAlcaniz, J.V., Dukhande, V.V., Donderis, J., Worby, C.A., Marina, A., Criado, O., Koller, A., Rodriguez De Cordoba, S., Gentry, M.S. and Sanz, P., Laforin, a dual-specificity phosphatase involved in Lafora disease, is phosphorylated at Ser25 by AMP-activated protein kinase. Biochem J, 2011. 439(2): p. 265-275.

57. McBride, A., Ghilagaber, S., Nikolaev, A. and Hardie, D.G., The glycogen-binding domain on the AMPK beta subunit allows the kinase to act as a glycogen sensor. Cell Metab, 2009. 9(1): p. 2334.

58. Li, X., Wang, L., Zhou, X.E., Ke, J., de Waal, P.W., $\mathrm{Gu}, \mathrm{X}$. , Tan, M.H., Wang, D., Wu, D., Xu, H.E. and Melcher, K., Structural basis of AMPK regulation by adenine nucleotides and glycogen. Cell research, 2014.

59. Jensen, J., Rustad, P.I., Kolnes, A.J. and Lai, Y.C., The role of skeletal muscle glycogen breakdown for regulation of insulin sensitivity by exercise. Frontiers in physiology, 2011. 2: p. 112. 




\section{Chapter 4}

\section{The interaction between AMPKß2 and the PP1- targeting subunit $\mathrm{R} 6$ is dynamically regulated by intracellular glycogen content}

Yvonne Oligschlaeger, Marie Miglianico, Vivian Dahlmans, Carla Rubio-Villena,

Dipanjan Chanda, Maria Adelaida Garcia-Gimeno, Yilin Liu, J. Willem Voncken, Joost J.F.P. Luiken, Jan F.C. Glatz, Pascual Sanz, Dietbert Neumann 


\section{Abstract}

AMP-activated protein kinase (AMPK) was suggested to coordinate muscular glucose uptake and glycogen metabolism in response to metabolic stress. We previously showed that glucose deprivation induces autophosphorylation of AMPK $\beta$ at threonine-148 (Thr-148), which prevents the binding of AMPK to glycogen. Furthermore, in MIN6 cells AMPK $\beta 1$ binds to R6 (PPP1R3D), a glycogen-targeting subunit of protein phosphatase 1 (PP1), thereby regulating the glucose-induced inactivation of AMPK. Here, we further investigated the interaction of R6 with AMPK $\beta$ and the possible dependency on Thr-148 phosphorylation status. Yeast two-hybrid analyses and co-immunoprecipitation of the overexpressed proteins in HEK293T cells revealed that both AMPK $\beta 1$ and $\beta 2$ wild-type (WT) isoforms bind to R6. The interaction with R6 was stronger with the muscle-specific $\beta 2$-WT and required association with the substrate-binding motif of R6. When HEK293T cells or C2C12 myotubes were cultured in high-glucose medium, AMPK $\beta 2$-WT and R6 weakly interacted. In contrast, glycogen depletion significantly enhanced this protein interaction. Mutation of AMPK $\beta 2$ Thr-148 prevented the interaction with R6 irrespective of the intracellular glycogen content. Treatment with the AMPK activator oligomycin enhanced AMPK $\beta 2 /$ R6 interaction in conjunction with increased Thr-148 phosphorylation in cells grown in low glucose medium. These data are in accordance with R6 binding directly to AMPK $\beta 2$ when both proteins detach from the diminishing glycogen particle, which is simultaneous to increased AMPK $\beta 2$ Thr-148 auto-phosphorylation. Such model points to a possible control of AMPK by PP1-R6 upon glycogen depletion in muscle. 


\section{Introduction}

Muscular tissue, in particular skeletal muscle, is an important site for glucose storage. It has become evident that glucose disposal into glycogen is essential for coordinated glucose homeostasis, as conditions limiting glycogen synthesis are associated with, for instance, hyperglycemia and insulin resistance [1]. AMP-activated protein kinase (AMPK) is a metabolic energy sensor that mediates insulin-independent GLUT4 translocation to the plasma membrane resulting in increased glucose uptake [2]. Therefore, AMPK activation could normalize blood glucose levels in type 2 diabetic patients. In response to various cellular stresses (e.g., contraction, nutrient-deprivation), AMPK is activated and modulates downstream targets to induce catabolic, ATPproducing processes and inhibit anabolic, ATP-consuming processes thereby restoring energy homeostasis. AMPK consists of three subunits, the catalytic $\alpha$ subunit, and two regulatory $\beta$ and $\gamma$ subunits; the latter are essential for regulating AMPK activity, as well as subcellular localization. AMPK subunits occur in different isoforms $(\alpha 1, \alpha 2 ; \beta 1$, $\beta 2 ; \gamma 1, \gamma 2, \gamma 3$ ) partly showing a tissue specific expression pattern. Namely, $\beta 2$ is the predominant isoform found in heart and skeletal muscle [3, 4].

AMPK is known to shuttle between the nucleus [5, 6] and the cytoplasm [7, 8], suggesting that AMPK exerts compartment-specific effects in order to monitor and coordinate complex cell biological processes. The $\beta$-subunit carbohydrate-binding module (CBM) allows AMPK to associate with glycogen $[9,10]$, where it interacts with other glycogen-binding proteins including glycogen synthase (GS) [10] and glycogen phosphorylase (GP) [9]. Exercise-induced AMPK activation inactivates GS via Ser7 phosphorylation in skeletal muscle, thereby preventing the glycogen synthesis process from further consuming ATP under conditions of cellular stress. Notably, $\beta 2$ shows higher affinity for glycogen compared to $\beta 1$ [11].

The regulation of the activity status of localized AMPK is dependent on allosteric activation/repression and the action of upstream kinases capable of phosphorylating threonine-172 (Thr-172) in the catalytic $\alpha$ subunit (e.g. LKB1, TAK1, CaMKK2). Several protein phosphatases (e.g. PP1, PP2A, PP2C) dephosphorylate Thr-172 thus leading to AMPK inactivation. PP1 is an important protein phosphatase in mammalian cells that is involved in proper coordination of glycogen metabolism by dephosphorylating target enzymes such as GS and GP. Recruitment of PP1 to its target substrates and to glycogen occurs by means of its glycogen-targeting proteins, such as PTG/R5 (PPP1R3C) [12] or R6 (PPP1R3D) [13, 14]. Insight into PP1-mediated AMPK regulation came from our previous study [15], which showed that R6 physically interacts with the AMPK $\beta 1$ subunit resulting in glucose-induced AMPK dephosphorylation by the PP1-R6 complex. Furthermore, we found that the CBM domain within the AMPK $\beta 1$ subunit is required 
for interaction with R6, as substitution of glycine-147 for arginine (G147R) resulted in total loss of AMPK/R6 interaction [15]. These results were consistent with parallel studies in yeast showing that Snf1 is regulated by glucose, and that Gal83 via its CBM interacts with Reg1, the orthologs of AMPK $\beta$ and R6, respectively [16], thus pointing to an evolutionary conserved mechanism.

We recently reported that autophosphorylation of Thr-148 in the AMPK $\beta$-subunit interferes with the recruitment of AMPK to glycogen, suggesting that AMPK-glycogen localization is tightly regulated and linked to glycogen storage [17]. Given the direct binding of AMPK $\beta 1$ to R6 and the observed loss of this interaction using the AMPK $\beta 1$ G147R mutant, we hypothesized a role for AMPK $\beta$ Thr-148 phosphorylation. In this current study, we provide insight into the binding of AMPK $\beta 2$ to R6 in relation to glycogen content and AMPK $\beta 2$ Thr-148 phosphorylation. Our results indicate that the AMPK $\beta 2 /$ R6 interaction is dynamically controlled by glycogen content.

\section{Experimental procedures}

\section{Plasmids}

For expression in mammalian cells, the cDNA of full-length AMPK $\beta 1$ and AMPK $\beta 2$ was amplified by PCR and ligated in frame into the pmCherry expression vector (Clontech) via EcoRI and SalI restriction sites, as previously described [17]. mCherryAMPK $\beta 2$ constructs bearing a threonine-to-aspartate mutation on residue 148 (T148D) was generated using the Quick-change site-directed mutagenesis kit (Stratagene). The pcDNA3 constructs for expression of AMPK $\gamma 1$ and myc-AMPK $\alpha 1$ were kindly provided by Dr. D. Carling (Imperial College London, London, United Kingdom). The corresponding ORF of AMPK 32 -T148D mutant was subcloned into pBTM116 to allow for expression in yeast (LexA-AMPKR2-T148D). The pFLAG-R6 construct for mammalian expression of R6-WT and R6-mutants (R6-RARA, -RAHA, -WDNAD and -WANNA) were generated as previously described, and the pFLAG was used as empty vector $(\mathrm{EV})$ control $[15,18]$.

Other plasmids used for yeast two-hybrid analyses were pGADT7- $\Phi(G A D$, empty plasmid), pGADT7-R6 (GAD-R6), pBTM-R6 (LexA-R6), pBTM-R6-RARA (LexA-R6RARA), pBTM-R6-RAHA (LexA-R6-RAHA), pBTM-R6-WANNA (LexA-R6-WANNA), pBTM-R6-WDNAD (LexA-R6-WDNAD), pGADT7-AMPK $\beta 1$ (GAD-AMPK $\beta 1$ ), pGADT7AMPK $\beta 2$ (GAD-AMPK $\beta 2$ ), pBTM-AMPK $\beta 1$ (LexA-AMPK $\beta 1$ ), pBTM-AMPK $\beta 2$ (LexAАMPK $\beta 2)[15,18-20]$.

For retroviral overexpression and optimal detection of exogenous $\beta 2$, full-length $\beta 2$ WT was amplified using PCR primers containing an optimized tetra-cysteine sequence (FlAsH-tag; FLNCCPGCCMEP). First, the corresponding ORF was subcloned into the SalI and NotI site of the mammalian pmCherry- $\beta 2$ construct, replacing mCherry- $\beta 2$ 
-WT with FlAsH- $\beta 2$-WT. Second, FlAsH- $\beta 2$-WT was amplified and subcloned into the EcorI and SalI site of the retroviral pBabe-puromycin retroviral backbone (kindly provided by Dr. G. Nolan, Stanford University, CA).

For retroviral co-expression of R6, the corresponding ORF (R6-WT) was amplified by PCR and subcloned into the BstxI and SalI restriction sites of the pBabe-hygromycin retroviral backbone (kindly provided by Dr. G. Nolan, Stanford University, CA). The pBabe-hygromycin EV was used as control. Primer sequences are available from the author upon request. All of the constructs were verified by DNA sequencing.

\section{Cell culture}

The human embryonic kidney 293T cell line (HEK293T) was cultured in DMEM with high glucose (25 mM) (Gibco), supplemented with $10 \%(\mathrm{v} / \mathrm{v})$ heat-inactivated fetal calf serum (iFCS, Bodinco BV, Alkmaar, The Netherlands) and penicillin/streptomycin (Invitrogen), unless otherwise stated. For transient transfections, HEK293T cells were seeded to $30 \%$ confluence in 6-well plates (Greiner Bio-one) $24 \mathrm{~h}$ before transfection. For determination of protein-protein interaction, cells were co-transfected with AMPK ( $\alpha 1$-myc, $\gamma 1$, and mCherry- $\beta 1$-WT or mCherry- $\beta 2-W T / \beta 2-T 148 D)$, and/or FLAG-R6 (R6-WT/R6-RARA/R6-RAHA/R6-WANNA/R6-WDNAD) or FLAG empty vector (EV) plasmid DNA using Lipofectamine 2000 (Invitrogen) in antibiotic-free culture medium. Six to eight hours after transfection, transfection medium was replaced by normal growth medium. At 48-72 h after transfection and continuous culturing (i.e. without change of medium), cells were harvested for glycogen or subjected to immunoprecipitation and Western blotting.

In order to induce glycogen depletion, HEK293T cells growing in high glucose medium (DMEM $25 \mathrm{mM}$ glucose, $10 \% \mathrm{iFCS}$ ), were subjected to forskolin treatment (100 $\mu \mathrm{M}$, Sigma), low glucose medium (DMEM 3 mM glucose, $10 \%$ iFCS), or glucosedeprived medium (DMEM $0 \mathrm{mM}, 10 \% \mathrm{iFCS}$ ) for $16 \mathrm{~h}$. To activate AMPK, cells were serum-starved in high- (DMEM $25 \mathrm{mM}$ ) or low-glucose (3 mM) medium for $16 \mathrm{~h}$, and subsequently treated with oligomycin ( $3 \mu \mathrm{M}$, Sigma) for $1 \mathrm{~h}$.

The mouse skeletal muscle cell line C2C12 was kindly provided by Dr. R.C. Langen (Maastricht University, The Netherlands). C2C12 cells were continuously cultured to approximately $80 \%$ confluence in DMEM with high glucose (25 mM) (Gibco), supplemented with $10 \%$ (v/v) iFCS (Bodinco BV, Alkmaar, The Netherlands) and $1 \%$ penicillin/streptomycin (Invitrogen). For differentiation into myotubes, myocytes (75$85 \%$ confluence) were further grown in differentiation medium (DMEM $25 \mathrm{mM}$ glucose [Gibco], supplemented with $2 \%$ heat-inactivated horse serum [Invitrogen, Life Technologies] and $1 \%$ penicillin/streptomycin [Invitrogen]) for 4 - 5 days. Subsequently, cells were used in the experiment corresponding experiments. In order 
to induce glycogen depletion, myotubes were maintained in low glucose medium (DMEM 3 mM glucose, supplemented with $2 \%$ iFCS), for $16 \mathrm{~h}$. In order to activate AMPK, myotubes were maintained in high or low glucose for 16 hours as described above, and subsequently treated with oligomycin (5 $\mu \mathrm{M}$, Sigma) for $30 \mathrm{~min}$.

\section{Retroviral infections}

In order to study protein-protein interactions in C2C12 myotubes, growing cells were (co-)infected with AMPK $\beta 2-\mathrm{WT}$ and/or R6 retroviruses. Briefly, retroviral systems and Phoenix helper-free retrovirus producer cell lines were used as published before [2123]. Amphotropic retroviral supernatants were produced as previously described [17] Briefly, 24-48 hours after calcium phosphate/DNA transfection of producer cells, supernatants were harvested, filtered (0.45 micron filters; Corning, Germany) and used for infection of $\mathrm{C} 2 \mathrm{C} 12$ cells in presence of $4 \mu \mathrm{g} / \mathrm{ml}$ polybrene (Sigma). For infections, cells were incubated with virus particles for 6-8 hours and then allowed to recover for $48 \mathrm{~h}$ on fresh medium, before selection pressure was applied. Stably infected cells were selected 2 days post-infection with $\mu \mathrm{g} / \mu \mathrm{L}$ puromycin for 36-48 hours, and 7 days post-infection with $300 \mu \mathrm{g} / \mu \mathrm{L}$ Hygromycin B for 10 days preceding experiments.

\section{Immunoprecipitation and Western blotting}

Immunoprecipitation procedures were performed as previously described [17]. Briefly, exogenous myc-AMPK $\alpha 1$ was immunoprecipitated using a myc-tag antibody (9B11, Cell Signaling Technology, Beverly, MA), and exogenous R6 was immunoprecipitated using FLAG-tag antibody (F3165, Sigma), followed by incubation with protein G-Sepharose beads (GE Healthcare). Western blot analysis was carried out using the following primary antibodies: myc-tag, tAMPK $\alpha$, AMPK $\beta 1$, AMPK $\beta 2$, phosphoAMPK-T172 (all from Cell Signaling Technology, Beverly, MA), FLAG-tag (F3165, Sigma), R6 (AP13440a, Abgent), and phospho-AMPKß2-T148 [17]. R6 was detected using FLAG-tag (F3165, Sigma), unless otherwise stated. Detection was performed according to its primary antibody using anti-rabbit (Cell Signaling Technology) and anti-mouse (Dako), anti-sheep (Calbiochem) or anti-goat (SantaCruz) horse-radish peroxidase (HRP)-conjugated secondary antibodies, followed by chemiluminescence.

In order to investigate the role of glycogen, myc-AMPK $\alpha 1$ was immunoprecipitated using the myc-tag antibody after the addition of the glycogen-mimic $\beta$-cyclodextrin ( $5 \mathrm{mM}$, Sigma) for $1 \mathrm{~h}$ at $4{ }^{\circ} \mathrm{C}$. Subsequently, immune complexes were electrophoresed by SDS-PAGE and analyzed by Western blot analysis, as described above.

\section{Biochemical intracellular glycogen measurement}

Intracellular glycogen content was measured, as previously described [17]. Briefly, 
HEK293T cells (non-transfected or transfected) or stably-infected C2C12 myotubes were lysed in potassium hydroxide (30\%) and boiled at $70{ }^{\circ} \mathrm{C}$ for $30 \mathrm{~min}$. Subsequently, samples were cooled to $25{ }^{\circ} \mathrm{C}$ before sodium sulfate $(6 \%, \mathrm{w} / \mathrm{v})$ and ethanol $(99.5 \%$, $\mathrm{v} / \mathrm{v}$ ) were added at a 1:1:3 ratio. After thorough mixing, samples were rotated topover-top at $4{ }^{\circ} \mathrm{C}$ for $30-60 \mathrm{~min}$. The precipitate was collected by centrifugation at $5000 \mathrm{rpm}$ for $5 \mathrm{~min}$ at $4{ }^{\circ} \mathrm{C}$. To hydrolyze glycogen, pellets were dissolved in $1 \mathrm{M} \mathrm{HCl}$ and boiled at $100{ }^{\circ} \mathrm{C}$ for $2 \mathrm{~h}$. Samples were cooled before neutralization using $2 \mathrm{M}$ $\mathrm{NaOH}$. Hydrolysates were used for glucose determination using a glucose (GO) assay kit (Sigma), according to the manufacturer's instructions.

Yeast two-hybrid (Y2H) analyses

Yeast two-hybrid analysis was performed as previously described [15, 19]. Briefly, interaction analysis used yeast THY-AP4 strain (MATa, ura3, leu2, lexA::lacZ::trp1, lexA::HIS3, lexA::ADE2) co-transformed with the indicated combination of plasmids (see above). Transformants were grown in selective $4 \%$ glucose synthetic complete medium lacking the corresponding supplements to maintain selection for plasmids. The strength of the interaction was determined by measuring $\beta$-galactosidase activity in permeabilized yeast cells and expressed in Miller units. In all Y2H analyses similar protein levels were obtained from the expression constructs, as verified in the crude extracts from the different yeast transformants.

\section{Statistical analysis}

All bar graph data are presented as mean \pm SEM, unless otherwise stated. Statistical differences were evaluated using unpaired Student's $t$-test and statistical analysis software Prism 4 (GraphPad Software, Inc.). P-values equal to or less than 0.05 were considered statistically significant.

\section{Results}

\section{R6 preferentially interacts with $A M P K \beta 2$}

We previously demonstrated that R6 (PPP1R3D), one of the glycogen-targeting subunits of the protein phosphatase 1 (PP1), physically interacts with AMPK $\beta 1$ in MIN6 pancreatic $\beta$ cells [15]. To verify AMPK $\beta 1 /$ R6 interaction in HEK293T cells, we overexpressed myc-tagged AMPK heterotrimers (myc- $\alpha 1$, mCherry- $\beta 1, \gamma 1$ ), together with FLAG-tagged R6 (FLAG-R6) or empty vector control (EV). Physical interaction between AMPK $\beta 1$ and R6 was assessed by immunoprecipitation (IP) of AMPK from cells that were routinely cultured under glucose-rich conditions (25 mM glucose) (Fig. 1A). Pull-down of myc-AMPK $\alpha 1$ resulted in co-immunoprecipitation of both mCherry- 
A

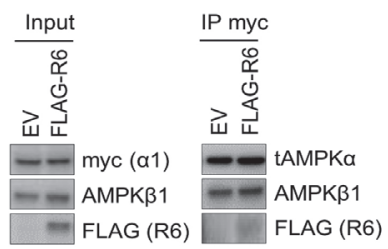

B

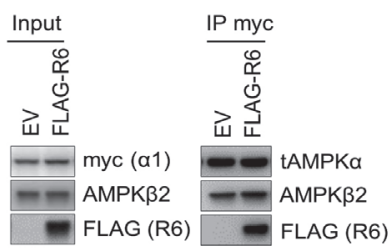

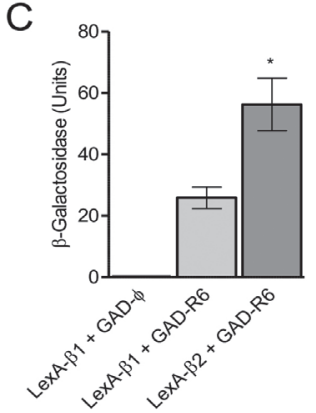

Figure 1. R6 preferentially interacts with AMPK $\beta 2$.

$(\mathrm{A}, \mathrm{B}) \mathrm{HEK} 293 \mathrm{~T}$ cells transiently overexpressed FLAG-R6 and heterotrimers of AMPK $\beta 1$ (myc- $\alpha 1, \gamma 1$, mCherry-AMPK $\beta 1$ ) (A) or AMPK $\beta 2$ (myc- $\alpha 1, \gamma 1$, mCherry-AMPK $\beta 2$ ) (B). FLAG empty vector (EV) was used as control. Cells were continuously cultured under high glucose $(25 \mathrm{mM})$ conditions and harvested $56 \mathrm{~h}$ post-transfection. Interaction between AMPK $\beta 1 / 2$ and R6 was assessed by immunoprecipitating the heterotrimeric AMPK complex from $800 \mu \mathrm{g}$ of lysate using the myc-tag antibody. Western blots were assessed using the indicated antibodies. Representative Western blots are shown. (C) Yeast THY-AP4 strain (see Experimental Procedures) was transformed with the indicated combination of plasmids. Transformants were grown in high glucose ( $4 \%$ glucose) containing medium and protein interactions were estimated by measuring the $\beta$-galactosidase activity. Empty vector pGADT7 (GAD- $\Phi$ ) served as control. Values correspond to means from at least 6 different transformants (bars indicate standard deviation). * $\mathrm{p}<0.001$ vs.LexA-AMPK $\beta 1-\mathrm{WT}+\mathrm{GAD}-\mathrm{R} 6$.

AMPKß1 (65 kD) and FLAG-R6, indicating formation of the heterotrimeric AMPK complex and the interaction with R6 (Fig. 1A). As expected, FLAG-R6 signal was absent in cells transfected with EV. Next, we investigated the interaction between musclespecific AMPK $\beta 2$ heterotrimers (myc- $\alpha 1$, mCherry- $\beta 2, \gamma 1$ ) and R6 (FLAG-R6) using cotransfection and immunoprecipitation in HEK293T. Again both proteins coimmunoprecipitated, thus pointing to occurrence of a physical interaction between R6 and AMPK $\beta 2$ (Fig. 1B). Interestingly, co-immunoprecipitation appeared stronger in the presence of the AMPK $\beta 2$ compared to $\beta 1$. These data were further confirmed by yeast-two-hybrid (Y2H) analyses: in Fig. 1C it is shown that R6 binds to both AMPK $\beta 1$ and $\beta 2$, while the interaction between $A M P K \beta 2$ and R6 is significantly stronger than the one between AMPK $\beta 1$ and R6. Combined, these findings suggest that R6 interacts more tightly with AMPK $\beta 2$ if compared to AMPK $\beta 1$.

\section{R6 interaction with AMPK $\beta 2$ requires its substrate-binding motif}

Recently, a number of functionally distinct protein domains have been identified in the R6 glycogen-targeting subunit of PP1 [18]. R6 is composed of domains that mediate binding to carbohydrates (via its CBM), binding to the PP1 catalytic subunit (PP1c; via the RVXF motif), and to the glycogen metabolism-related substrates of PP1 (via the 
highly conserved WDNND motif). To investigate whether and which of these motifs is involved in the interaction between the AMPK $\beta$ subunit and R6, we performed studies in HEK293T cells co-expressing AMPK $\beta 2$ complex (myc- $\alpha 1$, mCherry- $\beta 2, \gamma 1$ ) with FLAG-R6, the latter carrying various mutations corresponding to its protein motifs [18] (Fig. 2A, B). We again immunoprecipitated myc-AMPK $\alpha 1$ using a myc-directed antibody. Of note, endogenous R6 was not detected either in lysate or in immunoprecipitated material, thereby substantiating the need for FLAG-R6 overexpression and interaction between AMPK $\beta 2$ and R6 (Fig. 2B: EV condition). R6 carried various domain-specific mutations: in both R6-RARA and R6-RAHA mutants, the hydrophobic valine and phenylalanine residues within putative R6-RVXF motifs were substituted for alanine, allowing us to probe the role of the PP1-binding motif in AMPK 32 /R6-binding (Fig. 2B). R6-RARA, a mutant known to have lost its capacity to bind to endogenous PP1c but not to PP1-substrates [18], presented a similar AMPK $\beta 2$ binding profile compared to wild-type, non-mutated R6 (R6-WT; Fig. 2B; right panel). The R6-RAHA mutant that carried mutations in a domain close to the substrate-binding motif, however, had completely lost its ability to interact with AMPK (Fig. 2B; right panel). This data was nicely reproduced in a reciprocal $\mathrm{Y} 2 \mathrm{H}$ assay using AMPK $\beta 2$ as bait (Fig. 2C). Further, the substrate-binding motif (WDNND) was mutated to study its effect on AMPK $\beta 2 / R 6$ interaction. In one of the mutants, the two aspartate residues present within the WDNND motif were replaced by alanine (R6-WANNA mutant), whereas in the R6-WDNAD mutant, the second asparagine residue was replaced by alanine. While the R6-WDNAD mutant was still capable of interacting with AMPK $\beta 2$, the WANNA mutation abolished AMPK $\beta 2$ binding (Fig. 2B right panel, Fig. 2C). In good agreement, $\mathrm{Y} 2 \mathrm{H}$ analyses using AMPK $\beta 1$ or AMPK $\beta 2$ as bait confirmed that the binding of R6 to the AMPK $\beta$ subunits depended on the R6 substrate-binding motif being intact (Fig. 2C and 2D). Thus, we show that the AMPK $\beta / R 6$ interaction requires the R6 substrate-binding motif. In all subsequent experiments, we concentrated on AMPK $\beta 2$ / R6 interaction.

\section{AMPKß2-Thr-148 mutant shows reduced interaction with R6}

Previously, we showed that substitution of the glycine-147 residue for arginine (G147R) within the $\beta 1$-CBM domain abolished the interaction of AMPK with R6 [15], indicating that $A M P K \beta 1$ requires the CBM for interaction with R6. More recently, we demonstrated that autophosphorylation at the $\beta$-threonine-148 (T148) residue, centrally located in the CBM, prevents AMPK from binding to carbohydrates such as glycogen [17]. As we show here that R6 also interacts with AMPK $\beta 2$, we next investigated whether Thr-148 is required for AMPK $32 / \mathrm{R} 6$ interaction. To this end, a phosphomimicking T148D AMPK $\beta 2$ mutant was generated. Transiently transfected HEK293T 
A

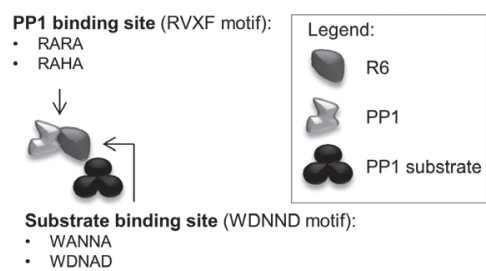

- WANNA

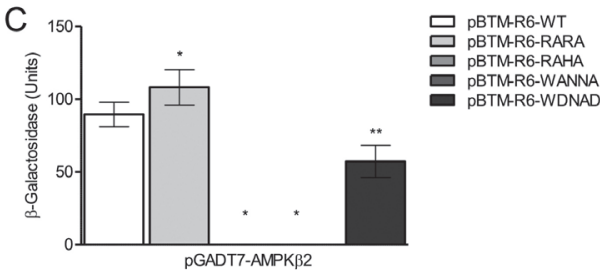

B

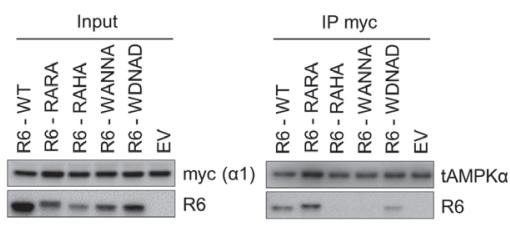

D

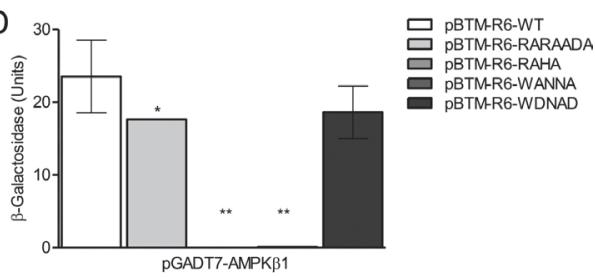

Figure 2. R6 interaction with AMPK $\beta 2$ requires its substrate-binding motif.

(A) Schematic model indicating the PP1- and substrate binding sites of R6, and its corresponding mutations. (B) HEK293T cells transiently co-expressing AMPK $\beta 2$ heterotrimers (myc- $\alpha 1, \gamma 1$, mCherry-AMPK $\beta 2$ ) and wild-type or mutant FLAG-R6 (R6-WT, R6-RARA, R6-RAHA, R6-WANNA, R6-WDNAD, respectively) were continuously cultured under high glucose $(25 \mathrm{mM})$ conditions and harvested $48 \mathrm{~h}$ post-transfection. FLAG empty vector (EV) was used as control. Interaction between AMPK $\beta 2$ and R6 was assessed by immunoprecipitating the heterotrimeric AMPK complex from $450 \mu \mathrm{g}$ of lysate using the myctag antibody. Western blots were assessed using the indicated antibodies. Representative Western blots are shown. (C, D) Yeast THY-AP4 strain (see Experimental Procedures) was transformed with GAD-AMPK $\beta 2$ (C) and GAD-AMPK $\beta 1$ (D) aplasmids and LexA-R6 forms plasmids. Transformants were grown in high glucose ( $4 \%$ glucose) containing medium and protein interactions were estimated by measuring the $\beta$-galactosidase activity. Values correspond to means from at least 6 different transformants (bars indicate standard deviation). ${ }^{*} \mathrm{p}<0.05$ and ${ }^{* *} \mathrm{p}<0.001$ vs. LexA-R6-WT.

cells co-expressing FLAG-R6 and AMPK heterotrimers (myc- $\alpha 1$ and $\gamma 1$ in combination with either mCherry- $\beta 2$-WT or mCherry- $\beta 2$-T148D), were cultured under high glucose conditions (25 $\mathrm{mM}$ ) and used for co-immunoprecipitation analysis. Impaired interaction between AMPK $\beta 2$ and R6 was observed in cells overexpressing mutant AMPK $\beta 2-T 148 D$ (Fig. 3A; right panel); relevantly, FLAG-R6 and mCherry- $\beta 2$ were expressed at comparable levels (Fig. 3A; left panel). Independent $\mathrm{Y} 2 \mathrm{H}$ analyses corroborated these findings: T148D mutation decreased the interaction between AMPK $\beta 2$ and R6 (Fig. 3B). Taken together, our data indicate that Thr-148 mutation into Asp results in loss of AMPK $\beta 2 /$ R6 interaction, suggesting that an intact Thr-148 residue is essential for the formation of the AMPK $\beta 2 / R 6$ complex under glucose-rich culturing conditions. 
A

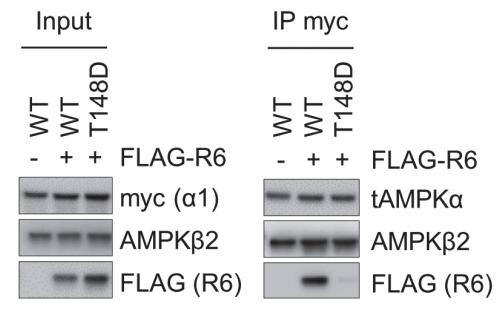

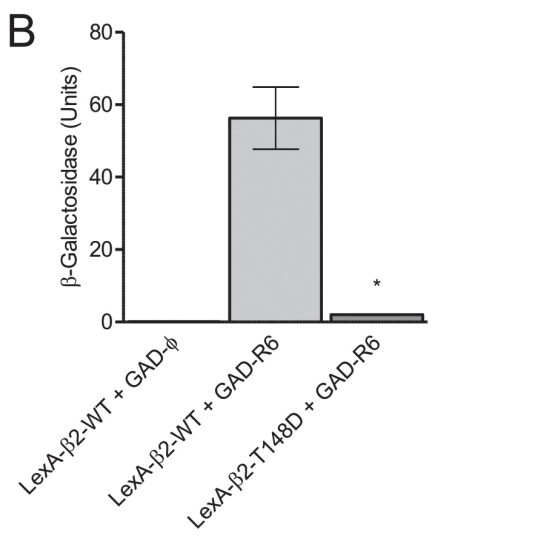

Figure 3. AMPKß2Thr-148 mutant shows reduced interaction with R6.

(A) HEK293T cells transiently transfected with myc- $\alpha 1, \gamma 1$ and mCherry-AMPK $\beta 2$ wild-type (WT) or T-148 mutant (T148D) were co-transfected either with FLAG-R6 (+), or FLAG empty vector (-) as control. Cells were cultured under high glucose $(25 \mathrm{mM})$ conditions, and harvested $56 \mathrm{~h}$ post-transfection. Interaction between АMPK $\beta 2$ and R6 was assessed by immunoprecipitating the heterotrimeric AMPK complex from $800 \mu \mathrm{g}$ of lysate using the myctag antibody. Western blots were assessed using the indicated antibodies. Representative Western blots are shown. (B) Yeast THY-AP4 strain was transformed with plasmids LexAAMPK $\beta 2-W T$ and T148D and GAD-R6 as indicated. Transformants were grown in medium containing high glucose ( $4 \%$ glucose) and protein interactions were estimated by measuring the $\beta$-Galactosidase activity. Empty vector pGADT7 (GAD- $\Phi$ ) served as control. Values correspond to means from at least 6 different transformants (bars indicate standard deviation). ${ }^{*} \mathrm{p}<0.001$ vs.LexA-AMPK $\beta 2$-WT + GAD-R6.

\section{Glycogen negatively regulates the interaction between $A M P K \beta 2$ and R6}

To investigate whether the interaction between AMPK $\beta 2$ and R6 is responsive to variation in glycogen level, we aimed to deplete cellular glycogen content. Hence, HEK293T cells were either pharmacologically treated with forskolin, a compound inducing glycogen breakdown [24], or glucose-deprived, after which glycogen content was assessed. Because R6 is known to have glycogenic properties [19], we included non-transfected control cells and compared these with cells that were either transfected with FLAG-R6 alone, or co-transfected with all three subunits of AMPK (myc- $\alpha 1$, $m C h e r r y-\beta 2-W T, \gamma 1)$. As expected, cells overexpressing FLAG-R6 showed high glycogen content (both in the absence and presence of co-overexpression of АMPK $\beta 2$ ): basal glycogen levels were 30-40-fold increased compared to non-transfected control cells (Fig. 4A). Both forskolin-treatment and glucose-deprivation significantly lowered the intracellular glycogen levels in all cell lines, as compared to control (Fig. 4A). To assess the effect of cellular glycogen content on AMPK $\beta 2 /$ R6 interaction, HEK293T cells overexpressing both FLAG-R6 and AMPK $\beta 2$ heterotrimers were either treated with forskolin or glucose-deprived for $16 \mathrm{~h}$. Reduction of cellular glycogen content, either by 
A

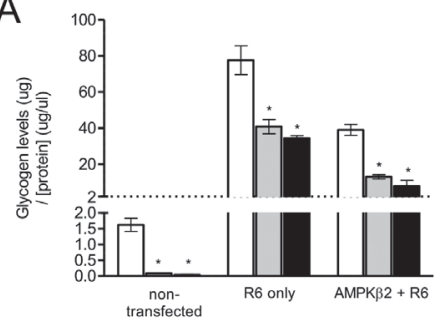

C

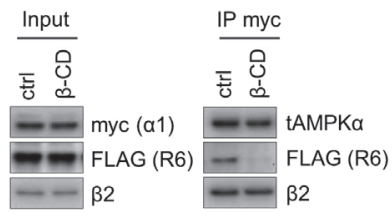

B

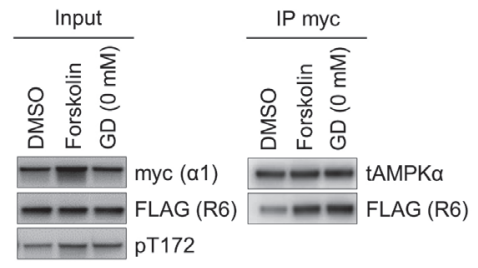

Figure 4: Glycogen negatively regulates the interaction between AMPK $\beta 2$ and R6.

(A, B) HEK293T cells either non-transfected, transiently transfected with FLAG-R6 alone, or co-transfected with FLAG-R6, myc- $\alpha 1, \gamma 1$ and mCherry-AMPK $\beta 2$-WT were cultured under high glucose $(25 \mathrm{mM})$ conditions. Prior to lysis, cells were treated with forskolin $(100 \mu \mathrm{M})$ or DMSO (control) under high glucose conditions for $16 \mathrm{~h}$, or cells were glucose-deprived (GD, $0 \mathrm{mM}$ ). (A) Intracellular glycogen level was determined as described in the section Experimental Procedures. Levels were corrected for corresponding protein concentrations. * $\mathrm{p}<0.05$ vs. corresponding DMSO controls; $\mathrm{n}=2$. (B) Heterotrimeric AMPK was immunoprecipitated from $500 \mu \mathrm{g}$ of lysate using the myc-tag antibody, followed by Western blot analysis using the indicated antibodies. Representative Western Blots are been shown. (C) HEK293T cells were transiently co-transfected as indicated and harvested 56 h posttransfection. Cells were continuously grown under high glucose $(25 \mathrm{mM})$ conditions. $\beta$-Cyclodextrin $(\beta$-CD) was added prior to immunoprecipitation. Representative Western blots are shown.

forskolin or glucose-deprivation, substantially enhanced the interaction between AMPK $\beta 2$ and R6 (Fig. 4B; right panel); as total levels of AMPK and R6 did not change within the experimental time frame (Fig. 4B; left panel), this ruled out any possible interference by altered expression. Collectively, our data suggest that the interaction between AMPK $\beta 2$ heterotrimers and R6 is inversely correlated to cellular glycogen level.

To independently examine a possible direct effect of glycogen on the AMPK/R6 binding, we next used $\beta$-cyclodextrin ( $\beta$-CD), a model-sugar that mimics glycogen, in competitive binding-immunoprecipitation experiments, with lysate issued from HEK293T cells cultured under high or low glucose. Addition of $\beta$-CD completely disrupted the interaction between AMPK $\beta 2$ and R6 (Fig. 4C; right panel). Combined, this data suggests that under glucose-rich conditions glycogen disturbs AMPK $\beta 2 /$ R6 interaction, that glycogen interferes with and therefore weakens the interaction between AMPK $\beta 2$ and R6. 
AMPKR2-R6 interaction is enhanced in conjunction with increased AMPKß2 Thr148 phosphorylation

Next, we assessed the impact of Thr-148 phosphorylation on the glycogen-modulated AMPK/R6 interaction. To this end, we co-transfected HEK293T cells with AMPK heterotrimers (myc- $\alpha 1, \gamma 1$, and either mCherry- $\beta 2-W T$ or -T148D mutant) and FLAG-R6 and challenged the cells for $16 \mathrm{~h}$ with various concentrations of glucose. The expression of AMPK 32 -T148D mutant did not affect the glycogenic activity of FLAG-R6 in cells growing in high glucose conditions (Fig. 5A), indicating that glycogen production depends on the function of R6 and not on the interaction between R6 and AMPK $\beta 2$. Reduced availability of glucose ( $3 \mathrm{mM}$ or $0 \mathrm{mM}$ ) correlated with significantly decreased intracellular levels of glycogen (Fig. 5A) in both AMPK 32 -WT and -T148D expressing cells (Fig. 5A). Interestingly, we observed increased level of Thr-148 phosphorylation in $\beta 2$-WT immunoprecipitates upon lowering glucose conditions, although we did not find changes in the levels of AMPK-Thr-172 phosphorylation, neither in cellular lysates nor in the precipitates from $\beta 2$-WT or $\beta 2$-T148D expressing cells (Fig. 5B). Low glycogen content enhanced AMPK $\beta 2 /$ R6 interaction (Fig. 5B; $c f$. Fig. 4). Whereas AMPK $\beta 2-W T$ /R6 binding was readily detectable, T148D mutation completely abolished AMPK $\beta 2$ interaction with R6 (Fig. 5B; $c f$. Fig. 3). To dissociate AMPK from glycogen via T148 phosphorylation and study the effect thereof on AMPK/R6 interaction, we next exposed cells to the AMPK activator oligomycin in the context of high and low cellular glycogen. If glycogen content was low (i.e. $3 \mathrm{mM}$ glucose), oligomycin activated AMPK (Thr-172 phosphorylation; Fig. 5C), an effect that was overruled by high cellular glycogen content (i.e. $25 \mathrm{mM}$ glucose). Oligomycin-induced activation of AMPK and the consequential Thr-148 phosphorylation further enhanced the interaction of AMPK $\beta 2$ WT and R6 (Fig. 5C; right panel) under low glycogen conditions. This interaction was disrupted by high cellular glycogen or by T148D mutation (Fig. 5C). These results indicate that glycogen-depletion enhances both AMPK $\beta 2 / R 6$ interaction and phosphorylation of Thr-148. These results also indicate that physiological phosphorylation of AMPK $\beta 2$ at Thr-148 has an outcome different from that of AMPK $\beta 2$ T148D mutant: whereas the AMPK $\beta 2-T 148 \mathrm{D}$ mutant is not able to interact with R6, this interaction is improved under physiological conditions that enhance AMPK $\beta 2$ Thr148 phosphorylation (Fig. 5C).

AMPK 32 shows enhanced interaction with R6 upon glycogen depletion in C2C12 myotubes

Furthermore, we explored the significance of cellular glycogen with respect to AMPK $32 /$ R6 interaction in C2C12 myotubes, a more physiologically relevant model. 
A

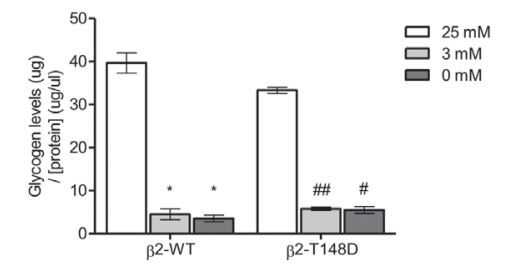

C
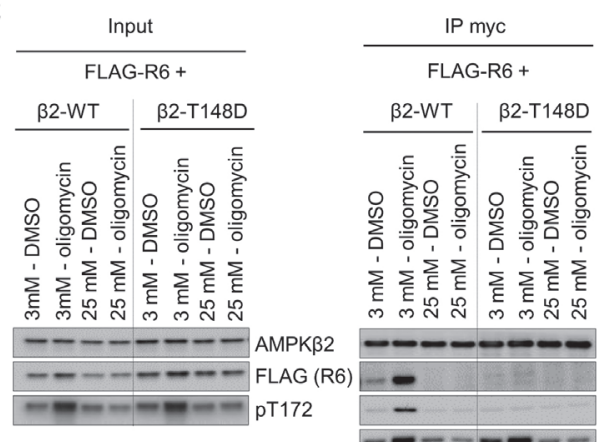

B
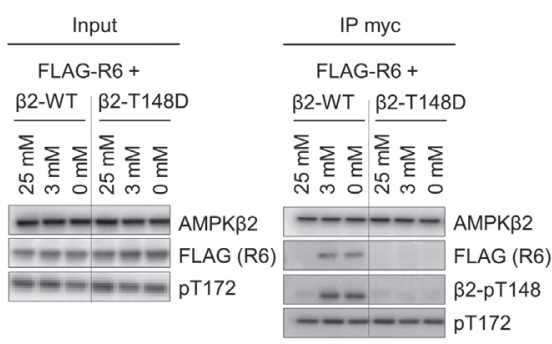

Figure 5. AMPKß2-R6 interaction is enhanced in conjunction with increased AMPKß2 Thr-148 phosphorylation.

(A, B) HEK293T cells co-expressed AMPK $\beta 2$ heterotrimers (myc- $\alpha 1, \gamma 1$, mCherry-AMPK $\beta 2$ -WT or -T148D) and FLAG-R6. Prior to lysis, cells were cultured under various glucose conditions ( $25 \mathrm{mM}, 3 \mathrm{mM}, 0 \mathrm{mM}$ ) for $16 \mathrm{~h}$. (A) Heterotrimeric AMPK was immunoprecipitated from $350 \mu \mathrm{g}$ of lysate using the myc-tag antibody, followed by Western blot analysis using the indicated antibodies. (B) Intracelllular glycogen levels were biochemically determined, and corrected for the corresponding protein concentrations. * $\mathrm{p}<0.01 \mathrm{vs}$. WT $25 \mathrm{mM}, \# \mathrm{p}<0.01 \mathrm{vs}$. T148D 25 mM, \#\# p<0.001 vs. T148D-25 mM; n=2. (C) Cells were serum-starved under low $(3 \mathrm{mM})$ and high $(25 \mathrm{mM})$ glucose conditions for $16 \mathrm{~h}$, prior to $1 \mathrm{~h}$ treatment with oligomycin $(3 \mu \mathrm{M})$ or DMSO (control). AMPK was immunoprecipitated from $500 \mu \mathrm{g}$ of lysate as described in panel A, followed by Western blot analysis using the indicated antibodies.

Since endogenous R6 was below detection level in C2C12 cells (data not shown), we co-expressed AMPK $\beta 2$ and FLAG-R6 in C2C12 myoblasts (Fig. 6). C2C12 cells were differentiated to myotubes and cultured under high (25 $\mathrm{mM}$ ) or low (3 $\mathrm{mM})$ glucose concentrations prior to oligomycin-treatment. Lowered glucose availability significantly decreased the myocellular glycogen content both in control cells and in cells expressing 32-WT (Fig. 6A). As expected, R6 overexpression enhanced the levels of glycogen under basal and treated conditions (Fig. 6A). Oligomycin clearly induced AMPK activation in total cell lysates, under both high and low glucose conditions (Fig 6B, left panel). However, as in HEK293T cells, oligomycin-mediated AMPK activation could only be detected in immunoprecipitates from cells that were cultured under low glycogen conditions (Fig. 6B; right panel). As expected, increases in interaction of 

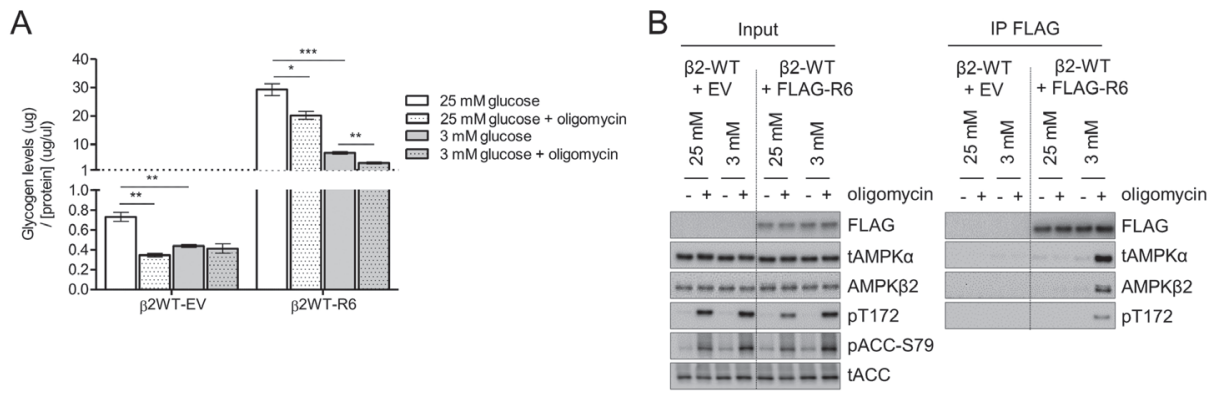

Figure 6. АMPK $\beta 2$ shows enhanced interaction with R6 upon glycogen depletion in C2C12 myotubes.

(A) C2C12 cells stably overexpressing both FLAG-R6 and wild-type AMPK 32 (WT) were differentiated into myotubes for 4-5 days, and subsequently incubated with high (25 mM) or low ( $3 \mathrm{mM}$ ) glucose for $16 \mathrm{~h}$, followed by 30 minutes treatment with oligomycin $(5 \mu \mathrm{M})$ or DMSO (control). Empty vector FLAG (EV) was used as control. Interaction between AMPK $\beta 2$ and R6 was assessed by immunoprecipitation using FLAG-tag antibody, followed by Western blot analysis using the indicated antibodies. Representative Western blots are shown. (B) Corresponding myocellular glycogen levels were determined using biochemical glycogen assay, as described in Experimental Procedures. ${ }^{*} \mathrm{p}<0.05,{ }^{* *} \mathrm{p}<0.01,{ }^{* * *} \mathrm{p}<0.001 ; \mathrm{n}=3$.

AMPK $32 /$ R6 were detected after immunoprecipitating FLAG-R6 from cells treated with oligomycin under low glycogen conditions (Fig. 6B; right panel). Taken together, in good agreement with our observations in HEKT293 cells, these data support the idea that cellular glycogen content controls AMPK $\beta 2 /$ R6 binding.

\section{Discussion}

We recently showed that activation of AMPK precedes AMPK $\beta$ Thr-148 autophosphorylation to preclude the AMPK complex from binding to glycogen [17]. Further data suggested a possible role for Thr-148 phosphorylation in glycogen turnover. R6 is a known glycogen-binding protein and PP1-targeting subunit which acts as a glycogenic driver [19]. In the current study, we demonstrate the involvement of Thr-148 in the dynamic AMPK $32 /$ R6 interaction that is governed by glycogen content.

R6 recruits PP1 phosphatase to its substrates (e.g., GS and GP) [18], thus playing a critical role in the regulation of glycogen metabolism. Most of the PP1-glycogentargeting subunits exert their actions through binding to PP1 via a conserved N-terminal PP1-binding motif (RVXF), and interaction with PP1-substrates via a conserved C-terminal substrate-binding (WXNXGNYX(L/I)) motif [18]. We have recently shown that R6 does utilize this conserved region to bind to its glycogenic substrates GS and GP [18]. In line with these results, our work indicates that AMPK $\beta / R 6$ interaction occurs 
also via the R6 substrate-binding motif (Fig. 2), as a mutation in this domain resulted in loss of interaction, a process that is independent of PP1 binding. Therefore, these results may suggest that R6 acts as a scaffold to bring AMPK $\beta 2$ in close proximity to the PP1 phosphatase, for instance, under conditions that require AMPK dephosphorylation and inactivation. However, we did not find evidence for R6/PP1-dependent AMPK dephosphorylation in our study under conditions where AMPK $32 /$ R6 interaction was enhanced. This may be attributed to the use of glycogen depletion and mitochondrial poisoning as triggers. Although we find these treatments as required to induce significant AMPK $\beta 2 / R 6$ interaction, their application necessarily goes along with elevated AMP levels and consequent protection from Thr-172 dephosphorylation [25].

In this work, we also aimed at better understanding the role of AMPK $\beta 2$ Thr-148 phosphorylation in glycogen metabolism. In accordance with earlier findings on AMPK $\beta 1$ [15], we show that AMPK $\beta 2 / R 6$ interaction is markedly increased under low glucose conditions, which correlated with decreased glycogen levels. Strong AMPK $\beta 2$ Thr-148 phosphorylation signals were only detected when glycogen levels were low. In this condition, oligomycin further enhanced the interaction between AMPK $\beta 2$ and R6, which augmented the phosphorylation of Thr-148. Therefore, Thr-148 phosphorylation is compatible with binding to R6 and even the interaction is enhanced in conjunction with this post-translational modification. In agreement with our earlier work, Thr-148 phosphorylation occurred if glycogen pools were diminishing. Thus, the autophosphorylation of Thr-148 by AMPK may be understood as a marker indicating detachment of AMPK from glycogen in conditions that challenge cellular glycogen level. Notably, in conjunction with lowered glycogen content, oligomycin-induced Thr-172 phosphorylation was higher. Therefore, consistent with our results obtained in HEK293T cells and C2C12 myotubes, glycogen may be interpreted as a storage site for inactive AMPK, which is in agreement with an earlier study that suggested AMPK inhibition by binding to glycogen branch points [26].

Similar to the observations in yeast [27], the equivalent AMPK $\beta 1-G 147 R$ mutation impaired the ability to interact with R6 [15]. Since AMPK $\beta$ Thr-148 is located adjacent to Gly-147, one would expect a similar loss of binding to R6 for Thr-148 mutant. In agreement, we report here that AMPK $\beta 2 /$ R6 interaction was completely lost in the AMPK $\beta 2$-T148D mutant. In our previous study, we found that T148D mutant did not bind glycogen [17]. Nevertheless, the molecular mechanism of explaining the loss of binding to R6 as a result of T148D mutation remains unclear, since Thr-148 phosphorylation occurs in conjunction with R6 interaction. There are at least two possible explanations: $(i)$ the T148D mutation causes a subtle modification in the conformation of the $\beta 2$ subunit resulting in the loss of interaction with R6, a process not shared by the physiological phosphorylation of this subunit; or (ii) the interaction 
of AMPK $32-W T$ with R6 that is observed during glycogen degradation evolves from their initial binding at glycogen. While we formally cannot rule out the former, there are arguments in support of the latter notion. Firstly, both AMPK 32 and R6 bind to glycogen via their respective CBM $[9,28]$ and weakly interact under conditions of high glycogen content. This interaction might be indirect, i.e. both proteins bind to glycogen independently of each other. It could be anticipated that localization of AMPK and R6 to glycogen would bring these proteins in closer proximity to each other and thus allow them to interact in a direct manner as a second step. This is indeed in accordance with our findings showing that depletion of intracellular glycogen resulted in enhanced AMPK $\beta 2 / R 6$ interaction. The treatment of AMPK $\beta 2 / R 6$ co-immunoprecipitations with $\beta$-CD accordingly showed that the AMPK $\beta 2 / R 6$ interaction was completely abolished under high intracellular glycogen content (Fig. 4C), whereas the interaction remained more stable if immunoprecipitations were performed from glucose-deprived cells (Fig. 4B). Similarly, in $\mathrm{Y} 2 \mathrm{H}$ analyses, low glucose media augmented the AMPK $\beta / \mathrm{R} 6$ interaction [29]. One step further, high glycogen content was found to decrease AMPK $\beta 2 / R 6$ interaction and thus glycogen may in fact rather perturb the direct binding between AMPK $\beta 2$ and R6. Although our experiments provide proof-of-principle for the inhibitory role of glycogen in AMPK $\beta / R 6$ interaction, the exact details, i.e. whether $\beta$-CD scavenges AMPK or R6 or both, are unknown. Yet, it might not be surprising if R6 prefers binding to glycogen over AMPK $\beta 2$ to further promote glycogenic activity, since the R6-substrate-binding motif partially overlaps with the CBM domain [18]. Therefore, these data support the idea that both AMPK 32 and R6, independently, are capable of binding to glycogen, thus facilitating the formation of multi-protein-glycogen complexes [30]. The alternative direct binding mode is triggered by lowered glycogen content as further discussed below.

AMPK plays an essential role in various biological processes involved in restoring cellular energy homeostasis. Tuning of such processes requires adequate regulation, and involves AMPK compartmentalization and complex formation with other proteins and substrates. For example, AMPK controls glycogen metabolism, as it is known to phosphorylate and inactivate GS in vitro and in vivo [31,32]. In yeast, it was reported earlier that Gal83 (Snf1 $\beta$-subunit ortholog) is involved in binding to Reg1 (PP1 glycogenic subuni ortholog) and studies in mouse pancreatic $\beta$ cells revealed that AMPK $\beta 1$ interacts with the PP1-glycogen targeting subunit R6 $[15,33]$. In the present study, we show that AMPK $\beta 2$ interacts more strongly with R6 if compared to AMPK $\beta 1$, pointing to a possible role for this interaction in skeletal muscle. The CBM domain of AMPK $\beta 2$ possesses higher binding affinity for carbohydrates such as glycogen than $\beta 1$ [11]. In a more detailed study the CBM domain of AMPK $\beta 2$ bound linear carbohydrates and single $\alpha 1,6$-branched carbohydrates 4-30 fold tighter in comparison with $\beta 1$ [34]. 
These data suggest that the AMPK $\beta 2$ isoform may be more important for coordination of glycogen storage and breakdown. Furthermore, given that AMPK $\beta 2$, as well as R6, are highly expressed in skeletal muscle $[3,35]$, a tissue active in controlling disposal of glucose into glycogen [36], it is likely that these proteins contribute to myocellular glycogen turnover. The $\mathrm{C} 2 \mathrm{C} 12$ murine cell line is widely used as an in vitro model of skeletal muscle [37], and we used these cells upon differentiation into muscle-like myotubes to investigate the dynamic interaction between AMPK $\beta 2$ and R6. Consistent with the findings of Bujak et al. that prolonged fasting induces the breakdown of skeletal muscle glycogen content [38], we demonstrate the reduction in myocellular glycogen levels as a result of glucose deprivation (Fig. 6A). Although we are currently lacking a direct proof, this breakdown of glycogen may cause AMPK $\beta 2$ and R6 to leave glycogen, as was reported for GS $[39,40]$. The possible mechanisms causing AMPK $\beta 2$ and R6 to leave the dwindling glycogen particle may include protein phosphorylation, such as Thr-148 phosphorylation, and the decreasing surface binding options. On a diminishing glycogen granule, we can indeed expect the concentration of potential interaction partners to increase dramatically, which may facilitate a direct binding between these proteins concomitant with their detachment from glycogen. In support of such notion, oligomycin-induced AMPK activation augments AMPK $\beta 2 /$ R6 interaction in skeletal muscle myotubes while further depleting glycogen and inducing Thr-148 phosphorylation (Fig. 6A), suggesting that these events are linked. In summary, we describe for the first time that R6 physically interacts with AMPK $\beta 2$ in C2C12 skeletal muscle myotubes, which is markedly enhanced if glycogen content is low. Vice versa, high availability of extracellular glucose and thus high intracellular glycogen are negative regulators of the direct $\mathrm{AMPK} \beta 2 / \mathrm{R} 6$ interaction. Given these findings, one would expect that dysregulation of AMPK $\beta 2 /$ R6 interaction may be linked to metabolic disorders such as type 2 diabetes or glycogen storage diseases, in which the glycogenic pathway is either impaired or hyper-activated, respectively.

Altogether, we envisage the following sequence of events: high glucose availability (Fig. 7A), mimicking an in vivo "fed" state, maintains cellular glycogen stores. Glycogenbinding proteins such as AMPK $\beta 2$ heterotrimers and R6 associate with glycogen, thereby forming multi-protein-glycogen complexes. AMPK $\beta 2-\mathrm{T} 148 \mathrm{D}$ remains cytosolic, and is thus prevented from "indirectly" interacting with R6. Upon nutrient deprivation (Fig. 7B), mimicking an in vivo "fasted" state, the AMP:ATP ratio rises, and the cellular glycogen level decreases. In response, AMPK $\beta 2$ heterotrimers and R6 are increasingly concentrated at the surface of the glycogen particle until forced to release and simultaneously engage in a direct interaction. Under these conditions, AMPK is turned into an active state, and due to an AMP-mediated conformational change, it is further prevented from dephosphorylation by protein phosphatases such as PP1 [25]. In 


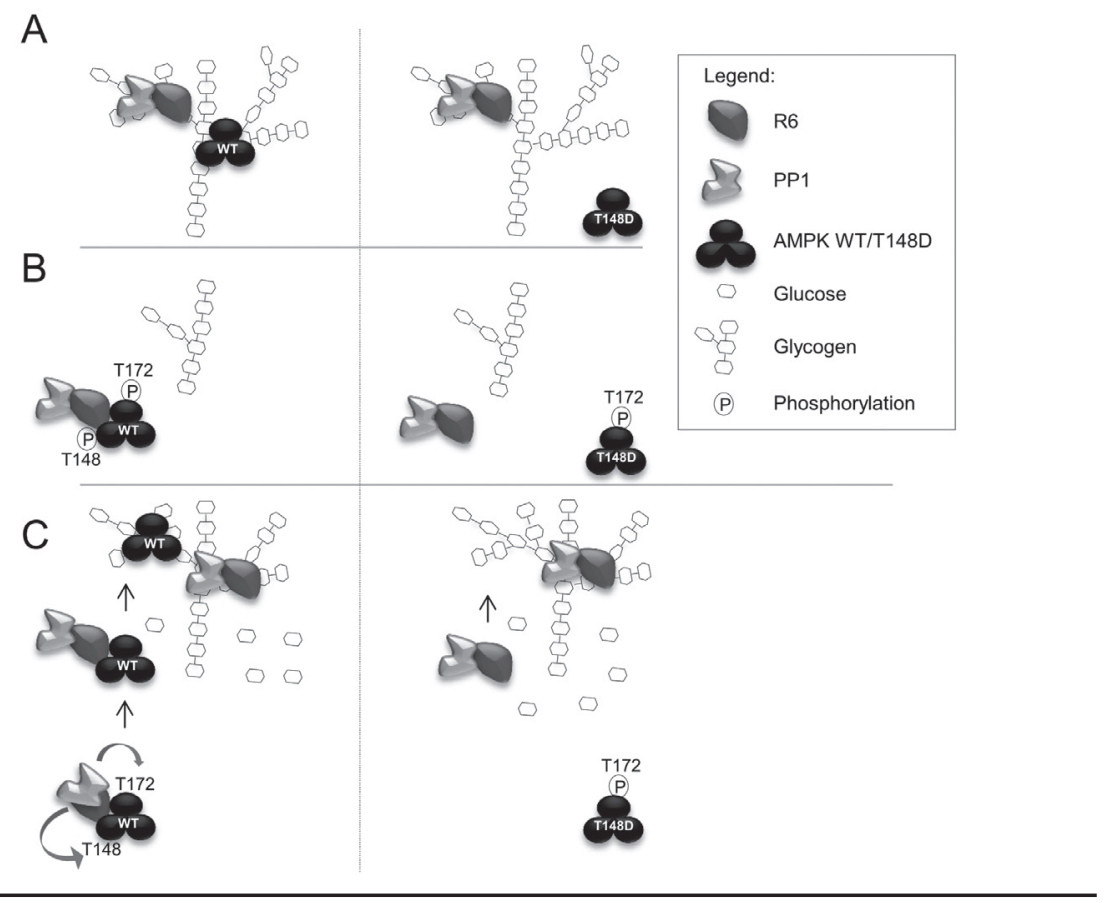

\section{Figure 7: Myocellular glycogen content dynamically regulates AMPK $\beta 2-\mathrm{R6}$} interaction.

(A) Regulation by high glucose availability/glycogen content, mimicking an in vivo "fed" state. (B) Regulation by low glucose availability/glycogen content, mimicking an in vivo "fasted" state. (C) Regulation by glucose re-availability/glycogen replenishment, mimicking an in vivo "re-fed" state. See main text for details.

addition, Thr-148 autophosphorylation prevents AMPK from returning to glycogen. Since the T148D mutant is not present at the glycogen particle during the departure of $\mathrm{R} 6$, these proteins are less likely to bind and form complexes. In response to glucose replenishment (Fig. 7C), mimicking an in vivo "re-fed" state,binding of ATP makes AMPK a better substrate for PP1-R6 mediated dephosphorylation. Thus, increases in intracellular glycogen reinforce the formation of a multi-protein-glycogen complex. The model provides with a possible explanation for AMPK $\beta 2 / R 6$ interaction in situations of low energy, where AMPK requires additional surveillance by PP1 to switch-off once cellular ATP is restored. Despite the fact that this model is supported by the presented results and experimental data from various studies, we admit that further investigation is required to advance it into solid knowledge. For instance, the molecular interplay of AMPK and PP1-R6 has not yet been addressed in cell free assays, which would allow for defined adenine nucleotide levels, and suitable genetic mouse models are lacking to further study AMPK functions in relation to PP1-R6 in tissues and the whole body context. 


\section{References}

1. Jornayvaz, F.R., Samuel, V.T. and Shulman, G.I., The role of muscle insulin resistance in the pathogenesis of atherogenic dyslipidemia and nonalcoholic fatty liver disease associated with the metabolic syndrome. Annual review of nutrition, 2010. 30: p. 273-290.

2. Kurth-Kraczek, E.J., Hirshman, M.F., Goodyear, L.J. and Winder, W.W., 5' AMP-activated protein kinase activation causes GLUT4 translocation in skeletal muscle. Diabetes, 1999. 48(8): p. 16671671.

3. Thornton, C., Snowden, M.A. and Carling, D., Identification of a novel AMP-activated protein kinase beta subunit isoform that is highly expressed in skeletal muscle. The Journal of biological chemistry, 1998. 273(20): p. 1244312450.

4. Chen, Z., Heierhorst, J., Mann, R.J., Mitchelhill, K.I., Michell, B.J., Witters, L.A., Lynch, G.S., Kemp, B.E. and Stapleton, D., Expression of the AMPactivated protein kinase beta1 and beta2 subunits in skeletal muscle. FEBS Lett, 1999. 460(2): p. 343-348.

5. McGee, S.L., Howlett, K.F., Starkie, R.L., Cameron-Smith, D., Kemp, B.E. and Hargreaves, M., Exercise increases nuclear AMPK alpha2 in human skeletal muscle. Diabetes, 2003. 52(4): p. 926-928.

6. Kodiha, M., Rassi, J.G., Brown, C.M. and Stochaj, U., Localization of AMP kinase is regulated by stress, cell density, and signaling through the $M E K->E R K 1 / 2$ pathway. American journal of physiology. Cell physiology, 2007. 293(5): p. C1427-1436.

7. Carling, D., Clarke, P.R., Zammit, V.A. and Hardie, D.G., Purification and characterization of the AMP-activated protein kinase. Copurification of acetyl-CoA carboxylase kinase and 3-hydroxy-3methylglutaryl-CoA reductase kinase activities. European journal of biochemistry / FEBS, 1989. 186(1-2): p. 129-136.

8. Kazgan, N., Williams, T., Forsberg, L.J. and Brenman, J.E., Identification of a nuclear export signal in the catalytic subunit of AMP-activated protein kinase. Mol Biol Cell, 2010. 21(19): p. 3433-3442.

9. Polekhina, G., Gupta, A., Michell, B.J., van Denderen, B., Murthy, S., Feil, S.C., Jennings, I.G., Campbell, D.J., Witters, L.A., Parker, M.W., Kemp, B.E. and Stapleton, D., AMPK $\beta$ Subunit Targets Metabolic Stress Sensing to Glycogen. Current Biology, 2003. 13(10): p. 867-871.

10. Hudson, E.R., Pan, D.A., James, J., Lucocq, J.M., Hawley, S.A., Green, K.A., Baba, O., Terashima, T. and Hardie, D.G., A Novel Domain in AMP-Activated Protein Kinase Causes Glycogen Storage Bodies Similar to Those Seen in Hereditary Cardiac Arrhythmias. Current Biology, 2003. 13(10): p. 861-866.

11. Koay, A., Woodcroft, B., Petrie, E.J., Yue, H., Emanuelle, S., Bieri, M., Bailey, M.F., Hargreaves,
M., Park, J.T., Park, K.H., Ralph, S., Neumann, D., Stapleton, D. and Gooley, P.R., AMPK beta subunits display isoform specific affinities for carbohydrates. FEBS Lett, 2010. 584(15): p. 3499 3503.

12. Fong, N.M., Jensen, T.C., Shah, A.S., Parekh, N.N., Saltiel, A.R. and Brady, M.J., Identification of binding sites on protein targeting to glycogen for enzymes of glycogen metabolism. The Journal of biological chemistry, 2000. 275(45): p. 3503435039.

13. Heroes, E., Lesage, B., Gornemann, J., Beullens, M., Van Meervelt, L. and Bollen, M., The PP1 binding code: a molecular-lego strategy that governs specificity. The FEBS journal, 2013. 280(2): p. 584-595.

14. Armstrong, C.G., Browne, G.J., Cohen, P. and Cohen, P.T.W., PPP1R6, a novel member of the family of glycogen-targetting subunits of protein phosphatase 1. FEBS letters, 1997. 418(1-2): $\mathrm{p}$. 210-214.

15. Garcia-Haro, L., Garcia-Gimeno, M.A., Neumann, D., Beullens, M., Bollen, M. and Sanz, P., The PP1R6 protein phosphatase holoenzyme is involved in the glucose-induced dephosphorylation and inactivation of AMP-activated protein kinase, a key regulator of insulin secretion, in MIN6 beta cells. FASEB J, 2010. 24(12): p. 5080-5091.

16. Sanz, P., Alms, G.R., Haystead, T.A. and Carlson, M., Regulatory interactions between the Reg1Glc7 protein phosphatase and the Snf1 protein kinase. Molecular and cellular biology, 2000. 20(4): p. 1321-1328.

17. Oligschlaeger, Y., Miglianico, M., Chanda, D., Scholz, R., Thali, R.F., Tuerk, R., Stapleton, D.I., Gooley, P.R. and Neumann, D., The Recruitment of AMP-activated Protein Kinase to Glycogen Is Regulated by Autophosphorylation. J Biol Chem, 2015. 290(18): p. 11715-11728.

18. Rubio-Villena, C., Sanz, P. and Garcia-Gimeno, M.A., Structure-Function Analysis of PPP1R3D, a Protein Phosphatase 1 Targeting Subunit, Reveals a Binding Motif for 14-3-3 Proteins which Regulates its Glycogenic Properties. PloS one, 2015. 10(6): p. e0131476.

19. Rubio-Villena, C., Garcia-Gimeno, M.A. and Sanz, P., Glycogenic activity of R6, a protein phosphatase 1 regulatory subunit, is modulated by the laforin-malin complex. The international journal of biochemistry \& cell biology, 2013. 45(7): p. $1479-1488$.

20. Gimeno-Alcaniz, J.V. and Sanz, P., Glucose and type $2 A$ protein phosphatase regulate the interaction between catalytic and regulatory subunits of AMP-activated protein kinase. Journal of molecular biology, 2003. 333(1): p. 201-209.

21. Kinsella, T.M. and Nolan, G.P., Episomal vectors rapidly and stably produce high-titer recombinant retrovirus. Human gene therapy, 1996. 7(12): p. 1405-1413. 
22. Morgenstern, J.P. and Land, H., Advanced mammalian gene transfer: high titre retroviral vectors with multiple drug selection markers and a complementary helper-free packaging cell line. Nucleic acids research, 1990. 18(12): p. 35873596.

23. Voncken, J.W., Niessen, H., Neufeld, B., Rennefahrt, U., Dahlmans, V., Kubben, N., Holzer, B., Ludwig, S. and Rapp, U.R., MAPKAP kinase $3 p K$ phosphorylates and regulates chromatin association of the polycomb group protein Bmi1. J Biol Chem, 2005. 280(7): p. 5178-5187.

24. Singh, P.K., Singh, S. and Ganesh, S., The laforinmalin complex negatively regulates glycogen synthesis by modulating cellular glucose uptake via glucose transporters. Molecular and cellular biology, 2012. 32(3): p. 652-663.

25. Suter, M., Riek, U., Tuerk, R., Schlattner, U., Wallimann, T. and Neumann, D., Dissecting the role of 5'-AMP for allosteric stimulation, activation, and deactivation of AMP-activated protein kinase. J Biol Chem, 2006. 281(43): p. 3220732216.

26. McBride, A., Ghilagaber, S., Nikolaev, A. and Hardie, D.G., The glycogen-binding domain on the AMPK beta subunit allows the kinase to act as a glycogen sensor. Cell metabolism, 2009. 9(1): p. 23-34.

27. Momcilovic, M., Iram, S.H., Liu, Y. and Carlson, M., Roles of the glycogen-binding domain and Snf4 in glucose inhibition of SNF1 protein kinase. The Journal of biological chemistry, 2008. 283(28): p. 19521-19529.

28. Machovic, M. and Janecek, S., Starch-binding domains in the post-genome era. Cellular and molecular life sciences : CMLS, 2006. 63(23): p. 2710-2724.

29. Garcia-Haro, L., Garcia-Gimeno, M.A., Neumann, D., Beullens, M., Bollen, M. and Sanz, P., Glucosedependent regulation of AMP-activated protein kinase in MIN6 beta cells is not affected by the protein kinase A pathway. FEBS letters, 2012. 586(23): p. 4241-4247.

30. Rybicka, K.K., Glycosomes the organelles of glycogen metabolism. Tissue\&Cell, 1996.

31. Carling, D. and Hardie, D.G., The substrate and sequence specificity of the AMP-activated protein kinase. Phosphorylation of glycogen synthase and phosphorylase kinase. Biochim Biophys Acta, 1989. 1012(1): p. 81-86.

32. Jorgensen, S.B., Nielsen, J.N., Birk, J.B., Olsen, G.S., Viollet, B., Andreelli, F., Schjerling, P., Vaulont, S., Hardie, D.G., Hansen, B.F., Richter, E.A. and Wojtaszewski, J.F., The alpha2-5'AMP-activated protein kinase is a site 2 glycogen synthase kinase in skeletal muscle and is responsive to glucose loading. Diabetes, 2004. 53(12): p. 30743081.
33. Mangat, S., Chandrashekarappa, D., McCartney, R.R., Elbing, K. and Schmidt, M.C., Differential roles of the glycogen-binding domains of beta subunits in regulation of the Snf1 kinase complex. Eukaryotic cell, 2010. 9(1): p. 173-183.

34. Mobbs, J.I., Koay, A., Di Paolo, A., Bieri, M., Petrie, E.J., Gorman, M.A., Doughty, L., Parker, M.W., Stapleton, D.I., Griffin, M.D. and Gooley, P.R., Determinants of oligosaccharide specificity of the carbohydrate-binding modules of AMP-activated protein kinase. Biochem J, 2015. 468(2): p. 245257.

35. Montori-Grau, M., Guitart, M., Garcia-Martinez, C., Orozco, A. and Gomez-Foix, A.M., Differential pattern of glycogen accumulation after protein phosphatase 1 glycogen-targeting subunit PPP1R6 overexpression, compared to PPP1R3C and PPP1R3A, in skeletal muscle cells. BMC biochemistry, 2011. 12: p. 57.

36. Ren, J.M., Marshall, B.A., Gulve, E.A., Gao, J., Johnson, D.W., Holloszy, J.O. and Mueckler, M., Evidence from transgenic mice that glucose transport is rate-limiting for glycogen deposition and glycolysis in skeletal muscle. The Journal of biological chemistry, 1993. 268(22): p. 1611316115.

37. Burattini, S., Ferri, P., Battistelli, M., Curci, R., Luchetti, F. and Falcieri, E., C2C12 murine myoblasts as a model of skeletal muscle development: morpho-functional characterization. European journal of histochemistry : EJH, 2004. 48(3): p. 223-233.

38. Bujak, A.L., Crane, J.D., Lally, J.S., Ford, R.J., Kang, S.J., Rebalka, I.A., Green, A.E., Kemp, B.E., Hawke, T.J., Schertzer, J.D. and Steinberg, G.R., AMPK Activation of Muscle Autophagy Prevents FastingInduced Hypoglycemia and Myopathy during Aging. Cell metabolism, 2015. 21(6): p. 883890.

39. Nielsen, J.N., Derave, W., Kristiansen, S., Ralston, E., Ploug, T. and Richter, E.A., Glycogen synthase localization and activity in rat skeletal muscle is strongly dependent on glycogen content. J Physiol, 2001. 531(Pt 3): p. 757-769.

40. Prats, C., Cadefau, J.A., Cusso, R., Qvortrup, K., Nielsen, J.N., Wojtaszewski, J.F., Hardie, D.G., Stewart, G., Hansen, B.F. and Ploug, T., Phosphorylation-dependent translocation of glycogen synthase to a novel structure during glycogen resynthesis. J Biol Chem, 2005. 280(24): p. 23165-23172. 



\section{Chapter 5}

\section{Displacing AMPK from glycogen by pharmacological targeting of its carbohydrate-binding module}

Marie Miglianico, Yvonne Oligschlaeger, Dipanjan Chanda, Kanin Wichapong, Will A. Coumans, Armand Jaminon, Ricardo Rodriguez-Calvo, Özge D. Özcete, Roy Schrijver, Xiaoqing Zhu, Ivo Bleylevens, Nynke M.S. van den Akker, Daniel G.M. Molin, Jan F.C. Glatz, Gerry A.F. Nicolaes, Dietbert Neumann 


\section{Abstract}

As a central player of energy metabolism, AMP-activated protein kinase (AMPK) is a well-established pharmacological target. Several identified AMPK-activating small molecules have fueled further research into their mechanism of action and substantiated the beneficial effects of AMPK activation in multiple disease settings. Here, we employ a novel screening protocol focusing on the carbohydrate-binding module (CBM) of AMPK. The procedure combines virtual ligand screening, a carbohydrate-binding competition assay, and assessment of the CBM subcellular localization pattern. Among the identified hit molecules, a cluster of similar compounds scored well in all screening steps. Specifically, two of the identified compounds had micromolar affinities for displacement of AMPK-CBM in cells. Surface plasmon resonance confirmed the direct binding of these two compounds to the CBM of AMPK. Remarkably, cellular application of a high-affinity compound led to increased AMPK activity and phosphorylation of a downstream target. Furthermore, the compound stimulated both glucose and fatty acid uptake in cultured cardiomyocytes, which could be reversed with the AMPK inhibitor Compound C. In summary, blocking access of AMPK to glycogen by targeting the carbohydrate-binding pocket with small molecules could be a novel alternative approach to AMPK activity modulation. 


\section{Introduction}

As a cellular hub that integrates many input signals (hormones, exercise, nutrient availability for instance) and balances energy expenditure and conservation, AMPactivated protein kinase (AMPK) is a recognized target for pharmaceutical modulation [1-5]. Initially identified as a target of interest in type 2 diabetes [2], AMPK is now being investigated in a vast area of cardiovascular diseases as well as in cancer and neurological diseases $[2,4,6]$.

Targeting AMPK with pharmacological agents is not a field in its beginning and no less than 3 different mechanisms have already been identified to pharmacologically activate AMPK $[1,2,5]$. This heterotrimeric kinase, constituted of a catalytic subunit $\alpha$ and two regulatory subunits $\beta$ and $\gamma$, is primarily activated by phosphorylation on the $\alpha$-Thr172 and is stimulated by an elevation of AMP:ATP ratio sensed by binding domains on its regulatory $\gamma$-subunit [6]. The first class of AMPK activators acts either indirectly by changing the adenine nucleotide ratio mainly via inhibition of the mitochondrial respiratory chain (e.g., phenformin, oligomycin) or by mimicking the AMP molecule and directly binding to the $\gamma$-subunit (e.g., 5-amino-4imidazolecarboxamide ribonucleoside [AICAR]) [2,6]. The activators of the second class (e.g., A-769662) bind between the regulatory subunit $\beta$ and the kinase domain of the catalytic subunit $\alpha$ [7], which induces a conformational change and allosterically activates AMPK [8] without necessarily changing its phosphorylation status $[9,10]$, and protects the Thr172 from dephosphorylation [8]. The compounds of the third class (e.g. PT-1) act by relieving the auto-inhibition on the kinase domain within the $\alpha$-subunit [11], although this mechanism of action has recently been doubted [12].

Over the years, AMPK regulation has emerged as a very fine and complex system, conveyed by post-translational modifications, subcellular localization and metabolite sensing [3]. Specifically, the AMPK $\beta$-subunit contains a carbohydrate-binding module (CBM) which is able to bind carbohydrates without having any catalytic activity [13, 14]. In cells, the CBM is capable of tethering AMPK to glycogen particles, and this binding is prevented by $\beta$-T148 autophosphorylation [15]. The physiological role of AMPK bound to glycogen was suggested to be the facilitation of the targeting of the kinase towards glycogen-bound partners [14]. Indeed, AMPK phosphorylates several glycogen-related targets, such as glycogen synthase [16], and spatial proximity would allow for a quick modulation of their activities. However, it was recently reported that in vitro binding of AMPK to glycogen inhibited its kinase activity [17], suggesting the existence of a glycogen-bound reserve of inactive AMPK in the cell. Although seemingly in disagreement, these two roles of glycogen-localized AMPK may be dependent on the degree of glycogen branching and thus separated in time or space [17]. The specific 
interplay of AMPK with glycogen, accordingly, remains an active area of research that furthers our understanding of AMPK's involvement in cellular glucose handling and glycogen metabolism.

Here we present a new screening protocol for identification of compounds that prevent AMPK from binding to glycogen, which combines a virtual ligand screening, a competition assay for binding of CBM to the carbohydrate $\beta$-cyclodextrin and a quantitative assessment of CBM subcellular localization. The most effective compounds resulting from this screening were further characterized for their binding affinity and cellular activity.

\section{Experimental procedures}

\section{Protein target preparation}

The X-ray structures of the isolated $\mathrm{CBM} \beta 1$ protein domain in complex with $\beta$-cyclodextrin (PDB ID: 1Z0M, resolution: $1.91 \AA$, [18]) and of the isolated apoprotein domain CBM $\beta 2$ (PDB ID: 2F15, resolution: $2.00 \AA$ ) were retrieved from the PDB-Redo database [19]. After removal of the co-crystallized $\beta$-cyclodextrin from the $1 \mathrm{Z0M}$ structure, the YASARA-WHAT IF twinset package [20] was used to add hydrogen and optimize both structures by energy minimization. The optimized structures were checked with the NIH structural analysis server which compiles 6 different programs [21-25] and were judged satisfactory after minor targeted residue optimizations. A shortmolecular dynamics simulation was run for 14 ns to sample possible conformations for both CBM proteins, with the md_run.mcr macro of the YASARA-WHAT IF Twinset package (with default parameters: AMBER03 as force field, 2.5 fs time step, and without constraint). Conformations of the snapshots taken every 25 ps were ranked according to volume of the carbohydrate-binding pocket. The 8 conformations with largest pockets that involved the loop formed by S100/T101 residues were selected for the virtual screening. For this step, icmPocketFinder (MolSoft) was used to quickly analyze all conformations. The selected conformers were then subjected to DoGSiteScorer [26] for further analysis of the targeted pocket. The sequence alignment was performed with ClustalX2 [27].

\section{Ligand database preparation}

A database of $\sim 800,000$ compounds composed of both the Express-Pick and the Combinatorial libraries of ChemBridge were retrieved (data from January 2012, hit2lead.com) and desalted with the Desalt script from Mobyle@RPBS webserver. FAFDrugs2 [28] was used to select drug-like compounds according to their ADME/tox properties with Lipinski's rule of 5 [29] and the filter for undesirable moieties moieties 
and pan-assay interference compounds (PAINs [30]). For rigid docking, the Omega2 software was used on the filtered library to generate multiple 3D conformations $(\sim 35$ conformers/compound; OpenEye Scientific Software, version 2.4). For flexible docking, a single 3D conformation was generated with Omega2 for the selected compounds, since that step includes ligand flexibility.

\section{Computer-based screen}

The in silico screen of the compound library followed an established pipeline of successive rigid and flexible dockings [31-33]. Briefly, the rigid-body docking software FRED (OpenEye Scientific Software, version 2.2.5) was first used to screen the complete collection of multiple compound conformers on 8 target protein conformations of $\mathrm{CBM} \beta 2$ to rapidly remove unlikely ligands from the library. The remaining best 240,000 compounds based on an averaged consensus score of the 8 conformations were selected for flexible docking with Surflex (BioPharmics LLC, version 2.514). Next, compounds were prioritized based on their averaged Combo scores. The bound poses of the best-scoring 1,000 compounds were visually inspected and the remaining top800 compounds were purchased from ChemBridge, diluted in DMSO and further tested in vitro. During the process and the following steps, the large amount of data was processed with Perl and Knime [34] using the CDK [35] and JChem Extension (ChemAxon, version 204) packages. Additionally, the webserver ChemMine was used to compare the compounds according to 2D similarity [36].

\section{Re-docking and binding free energy calculations}

Coordinates of the 3D structures of AMPK CBM $\beta 1$ in complex with $\beta$-cyclodextrin (PDB code 1Z0M) and apoprotein AMPK CBM 32 (PDB code 2F15) were downloaded from the PDB-Redo database as mentioned above. Ligands were docked into the binding pocket of the AMPK CBM $\beta 2$ by application of the Autodock Vina module plugin for PyMol [37]. The two isoforms were first superimposed and then coordinates of the $\beta$-cyclodextrin from the original X-ray structure of AMPK CBM $\beta 1$ (1Z0M.pdb) were selected as the center of the docking box for AMPK CBM 22 (2F15.pdb). The dimension of the box was set to $26.5 \times 26.5 \times 26.5 \AA$ to include all key residues at the binding pocket.

Binding free energy calculations such as MM/PB(GB)SA and recently developed QM/MM-GBSA were applied to first determine a likely binding mode of the potent compounds (6456019 and 6469172) and subsequently the best method was used to predict the binding affinity of these and other compounds as well. Generally, binding

free energy $\left(\Delta \mathrm{G}_{\text {binding }}\right)$ is computed based on the equations 


$$
\begin{aligned}
\Delta \mathrm{G}_{\text {binding }} & =\Delta \mathrm{H}_{\text {tot }}-\mathrm{T} \Delta \mathrm{S} \\
\Delta \mathrm{H}_{\text {tot }} & =\mathrm{E}_{\mathrm{MM} \mathrm{or} \mathrm{QM} / \mathrm{MM}}+\Delta \mathrm{G}_{\text {sol }}
\end{aligned}
$$

in which the $\Delta \mathrm{H}_{\text {tot }}$ term represents the binding enthalpy value and the $-\mathrm{T} \Delta \mathrm{S}$ term is the entropy change upon ligand binding. The enthalpy is derived by summation of the molecular mechanics (MM) or the hybrid quantum mechanics/molecular mechanics $(\mathrm{QM} / \mathrm{MM})$ interaction and the free energy of solvation $\left(\Delta \mathrm{G}_{\mathrm{sol}}\right)$. The $\mathrm{QM}$ method used in this study are AM1-DH+ and PM6-DH+ in which the dispersion and hydrogen bond correction are added [38]. The generalized Born model 8 (GB8) is used to calculate the free energy of solvation as it has been shown recently that the GB8 model performs better than other GB models [39]. The entropy change upon ligand binding was calculated by normal analysis. The calculated binding free energy $\left(\Delta G_{\text {cal. }}\right)$ either MM/ PB(GB)SA or QM/MM-GBSA was computed by application of the -MMPBSA.py module [40] implemented in AMBER12 [41] using the protocols as described previously [42, 43] except for those parameters mentioned above.

\section{Plasmids}

Constructs for mammalian transient overexpression of myc-tagged AMPKa1, AMPK $\beta 1$ and AMPK $\gamma 1$ were kindly provided by Dr. D. Carling (Imperial College London, London, United Kingdom). The construct of GFP-tagged CBM $\beta 1$ and $\operatorname{CBM} \beta 2$ for retroviral infection of mammalian cells are described elsewhere [15]. The CBM of the $\beta 2$ subunit (amino acid 67-163) was amplified by PCR and inserted into the pScherry2 expression vector (Delphigenetics) via the EcoRI and SalI restriction sites, resulting in bacterial expression constructs for SCherry-tagged CBM $\beta 2$.

\section{Cell-free in vitro screening}

SCherry-tagged CBM $\beta 2$ was expressed in Rosetta2 (DE3) Escherichia coli cells (Novagen). Bacteria were grown in auto-induction medium as described before [15], with the exception that the culture was upscaled to a 4-liter fermentor and run at $20^{\circ} \mathrm{C}$ for 30 hours. The protein was purified using metal affinity chromatography as previously described [15], and was subsequently loaded onto a column of epoxyactivated sepharose 5B (GE Healthcare) coupled to $\beta$-cyclodextrin. The column saturation was monitored using the red color of the SCherry tag. After column wash, the protein-resin complex was resuspended in a 50\% slurry with PBS supplemented with $0.5 \% \mathrm{v} / \mathrm{v}$ Tween 20 .

The principle of the competition assay is to test the ability of selected compounds to dissociate the protein from the immobilized $\beta$-cyclodextrin. The slurry is aliquoted in a 96-well plate where the compounds, dissolved in DMSO, are added 4 at a time, then for the active mixes only, one compound at a time to a final concentration of $100 \mu \mathrm{M}$ (final 
concentration of DMSO: $4 \%$ for mixes, $1 \%$ for singles). After settlement of the resin, the protein concentration found in the supernatant was measured with the Pierce BCA Protein Assay Kit (Life Technologies). The direct effect of the compound on BCA was measured in absence of protein/resin and subtracted to the first measure. This normalized absorbance was compared to effect on protein dissociation of vehicle (4\% for mixes, 1\% DMSO for singles) or $100 \mu \mathrm{M}$ maltoheptaose (Sigma Aldrich). We arbitrarily defined a difference of absorbance with the vehicle of 0.01 or higher, as a hit, if the associated two-tailed Student t-test gave a p-value below 0.05 (significant) or below 0.1 (near-significant).

\section{Cell-based screening}

C2C12 cells stably expressing the GFP-tagged CBM $\beta 1$ or $\beta 2$ were plated in a black 96-well plate (Greiner BioOne) with 10,000 cells/well in assay medium (DMEM with $4.5 \mathrm{~g} / \mathrm{L}$ glucose, L-Glutamine, $25 \mathrm{mM}$ Hepes, without phenol red [Life Technologies], supplemented with 100 units $/ \mathrm{ml}$ penicillin, $100 \mu \mathrm{g} / \mathrm{ml}$ streptomycin [Life Technologies] and with or without $5 \%$ heat-inactivated fetal calf serum [iFCS; Bodinco BV, Alkmaar, The Netherlands]). The following day, cells were stained with Hoechst ( $1 \mu \mathrm{g} / \mathrm{mL}$ final concentration, Life Technologies) for 30 minutes then treated for 8 hours at $37^{\circ} \mathrm{C}$ according to conditions described below, subsequently fixed with $2 \%$ paraformaldehyde for 10 minutes and finally kept in PBS supplemented with azide. Fixed cells were imaged for Hoechst and GFP with the high-content imaging system BD Pathway 855, with 16 pictures per well using a 40X magnification and autofocus for each view. Images were processed using the BD AttoVision software (version 1.7) to define cell circumferences using both nuclear staining and cell outline obtained with high gain imaging (typically >200) of the GFP signal. With low exposure images (typically $250 \mathrm{~ms}$ ), the GFP speckles, presumably caused by the concentrated binding of the GFPtagged CBM to intracellular glycogen particles, were delineated. Next, image data were imported in Kaluza software (version 1.2 Beckman Counter, Inc.) for image cytometric analyses, which gave the mean number of GFP speckle per cell for each well (typically 150-200 cells were imaged in every well). Alternatively, the percentage of cells showing the expected GFP pattern was retrieved for each well by applying gating for a minimal count of one GFP speckle per cell. During the first screen, cells were treated with $25 \mu \mathrm{M}$ compound $(0.25 \%$ DMSO) in plain DMEM without serum or antibiotics and supplemented with $10 \mu \mathrm{g} / \mathrm{mL}$ digitonin (Sigma-Aldrich) to facilitate cellular entry of the compounds. We arbitrarily defined a reduction in speckle number of $35 \%$, as compared with the control, as a hit. The dose response titrations were performed at varying compound concentrations $(0-100 \mu \mathrm{M})$ with a constant $1 \%$ DMSO in DMEM supplemented with $5 \%$ iFCS but without digitonin. The negative and positive control of 
speckle levels were the vehicle and $100 \mu \mathrm{M}$ forskolin (1\% DMSO), respectively. The dose response curves were fit to a dose-response model using GraphPad Prism software (GraphPad Software, Inc., version 5.03) to obtain IC50 measurements.

\section{Cell culture and treatment}

C2C12 cells were cultured in high-glucose medium (DMEM with $4.5 \mathrm{~g} / \mathrm{L}$ glucose, L-glutamine and pyruvate, supplemented with 10\% iFCS, 100 units/ml penicillin, and $100 \mu \mathrm{g} / \mathrm{ml}$ streptomycin) and differentiated for 7 days into skeletal myotubes with differentiation medium (high glucose DMEM, supplemented with $2 \%$ horse serum [Life Technologies], 100 units/ml penicillin, and $100 \mu \mathrm{g} / \mathrm{ml}$ streptomycin). Human embryonic kidney HEK293T and human hepatic HepG2 cells were cultured in the same high-glucose medium. Human fibroblast-derived near-haploid HAP1 cells, WT or knock-out for either AMPK- $\beta 1$ or - $\beta 2$, were cultured according to distributor guidelines (Horizon Genomics). All these cells were treated in their own DMEM but with only 5\% iFCS, except for undifferentiated C2C12 which were treated in assay medium (described above) with or without 5\% iFCS. Apart from screened compounds, treatment included oligomycin (Sigma-Aldrich), AICAR (Sigma-Aldrich), A-769662, and phenformin: the duration and concentrations are mentioned throughout the results. Following treatment, cells were quickly lysed and the lysates prepared for Western blotting. Alternatively, cells were fixed then stained with a glycogen antibody (courtesy of Dr. 0 . Baba, Tokyo Medical and Dental University, Tokyo, Japan) and imaged by confocal microscopy as described elsewhere [15].

\section{Cytotoxicity assay}

C2C12 were plated in assay medium at 10,000 cells/well of a 96-well plate. The following day, cells were treated with different doses of compound, vehicle (1\%DMSO), 1\% Triton-X or left untreated. After 2 hours of treatment, 3-(4,5-dimethylthiazol-2-yl)2,5-diphenyl tetrazolium bromide (MTT; Life Technologies) was added to the cells according to the manufacturer's recommendations. Cells were incubated for 4 hours at $37^{\circ} \mathrm{C}$ then DMSO was added and mixed until complete dissolution of the crystals. Absorbance was measured at $545 \mathrm{~nm}$ and normalized to that of untreated cells.

\section{Glucose and fatty acid uptake}

Glucose and fatty acid uptake were performed as previously described [44]. Briefly, cardiomyocyte HL1 cells were cultured according to the guidelines of the establishing team [45], and were treated in high glucose DMEM without iFCS. After overnight serum starvation, cells were pre-treated with $10 \mu \mathrm{M}$ Compound C (Dorsomorphin, abcamBiochemicals) or with vehicle for an hour, then $25 \mu \mathrm{M}$ of compound or vehicle 
(0.5\% DMSO final concentration) was added for an extra hour. Alternatively, $100 \mathrm{nM}$ of insulin was added for the last 20 minutes of treatment. After stimulation, a mixture of $\left[{ }^{3} \mathrm{H}\right]$ deoxyglucose and $\left[{ }^{14} \mathrm{C}\right]$ palmitate was added to the cells for 10 minutes then washed away. Glucose and fatty acid uptake was assessed by scintillation counting of the cell lysate [44]. Part of the final lysate was used for Western Blot.

\section{Surface plasmon resonance}

SCherry-tagged CBM $\beta 2$ protein was expressed and purified as described above and then immobilized on a SPR Sensorchip HC 1500 m (Xantecbioanalytics GmbH) by amine coupling to a density of 24,000 RU. The direct binding of the compounds was assessed at different concentrations in the running buffer (PBS, 0.05\% Tween 20, 5\% DMSO (v/v), pH 7.4) by surface plasmon resonance (Biacore T200; GE Healthcare). Sensorgrams were corrected for binding to an empty reference flow cell and were solvent corrected to account for slight changes in DMSO content. Data were analyzed with the BIAevaluation software and next compiled with GraphPad to obtain equilibrium dissociation constants $\left(\mathrm{K}_{\mathrm{D}}\right)$.

\section{Immunoprecipitation and kinase activity assay}

HEK293T cells were transfected with myc-tagged AMPK $\alpha 1$, AMPK $\gamma 1$ and either AMPK $\beta 1$ or $\beta 2$ using Lipofectamine 2000. Forty eight hours after transfection, cells were lysed and AMPK was immunoprecipitated using a myc-tag antibody (Cell Signaling Technology). For studying interaction partners of AMPK, before immunoprecipitation, the cell lysate was incubated with $5 \mathrm{mM} \beta$-cyclodextrin (Sigma-Aldrich), $25 \mu \mathrm{M}$ tested compounds or vehicle $(0.5 \%$ DMSO). Following immunoprecipitation, the samples were washed 3 times with lysis buffer before preparation of the proteins for Western Blot. In the case of the kinase assay, the immuniprecipitate was washed with the lysis buffer supplemented with high salt $(1 \mathrm{M} \mathrm{NaCl})$ to remove interacting partners, then 3 additional washes were performed with Tris Buffer $(80 \mathrm{mM}$ Tris with protease and phosphatase inhibitors, $\mathrm{pH}$ 7.4). The kinase assay was conducted following an adapted protocol from Promega for AMPK activity measurement using the ADP-Glo kit. Briefly, after the last wash, the kinase assay reactants were placed on the resin used to immunoprecipitate AMPK in a 2-fold concentrated manner to account for the bead volume. Final assay concentrations were $40 \mathrm{mM}$ Tris, $20 \mathrm{mM} \mathrm{MgCl}_{2}, 0.1 \mathrm{mg} / \mathrm{mL} \mathrm{BSA}$, $50 \mu \mathrm{M}$ DTT, $100 \mu \mathrm{M}$ AMP, $150 \mu \mathrm{M}$ ATP, $100 \mu \mathrm{M}$ SAMS, and either $100 \mu \mathrm{M}$ compound or corresponding vehicle ( $5 \%$ DMSO). The kinase assay was incubated at room temperature for 60 minutes with regular resuspension of the beads. Then $25 \mu \mathrm{L}$ of supernatant was transferred to a white 96-well plate to follow the ADP-Glo procedure (Promega) and measure with a luminometer (Glomax 96 Microplate Luminometer, 
Promega). The amount of ATP consumed by the kinase under different conditions was extrapolated from the standard curves as described in the Promega protocol.

\section{Statistical analysis}

All statistical analyses as well as graphics were prepared with the GraphPad Prism software (GraphPad Software, Inc., version 5.03).

\section{Results}

A screening coupling in silico and in vitro steps to identify glycogen-delocalizing molecules

Each of the three AMPK subunits exists as isoforms which partly exhibit tissuespecific expression profiles and different functions. In case of the $\beta$-subunit, the $\beta 1$ isoform is rather ubiquitously expressed, whereas the $\beta 2$ is more selectively expressed in muscle and liver tissues in humans $[3,46]$ and has a higher affinity towards carbohydrate compared to the $\beta 1$ isoform [47]. Considering that muscle and liver are very relevant target tissues in metabolic diseases [6] and contain high levels of glycogen, we aimed at targeting the $\beta 2$-subunit and focused on that isoform in the design of the screening. However, inspection of the X-ray structures and protein sequences of both CBM isoforms revealed a high sequence identity of $82 \%$ and sequence similarity of 93\% (Fig. 1A). Amino acid sequence conservation is especially high in the carbohydratebinding region. The main difference between the $\beta$-isoforms resides in the insertion of a threonine in position 101 (Thr101, according to numbering of the human $\beta 2$ sequence) enlarging the loop of the lower edge of the $\beta 2$-isoform binding pocket (Fig. 1B) [47]. In order to take advantage of this particularity, the selection of the protein conformations sampled with molecular dynamics was based on the inclusion of this extra space, as well as the more defined carbohydrate-binding region. Since the Thr101 points towards the outside of the pocket, its side chain is not part of the defined pocket. However the targeted pocket was lined with the side chains of the residues Ser93, Ser95, Trp99, Lys102, Gln124, Lys216, Trp133, Ser144, Leu146, Thr148 and Asn150, therefore it harbors both hydrophobic features and polar residues which may allow hydrogen bond formation. Among these protein conformations, the eight largest pockets occupied a volume between 254 and $305 \AA^{3}$ according to icmPocketFinder (261 to $371 \AA^{3}$ according to DoGSiteScorer). Despite their rather small volumes, the good chemical properties of the pocket allowed them to be judged fairly satisfactory druggable pockets by the DoGSiteScorer analysis, scoring 0.59 in average on a scale from 0 , undruggable, to 1 , druggable. Validated carbohydrate-binding pockets are known to score quite poorly in that kind of analysis, which means that a score around 
A

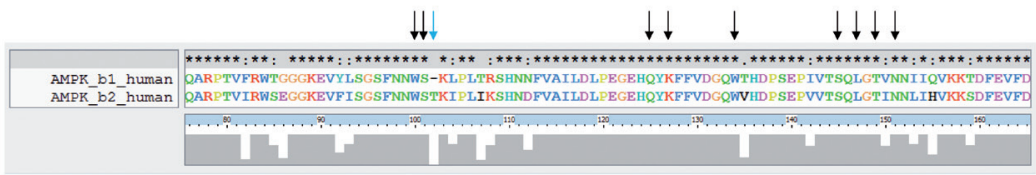

$\mathrm{B}$
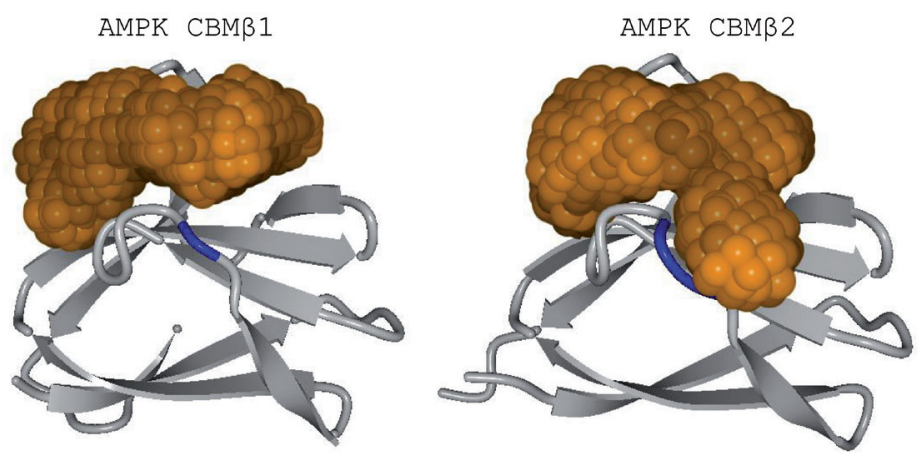

C

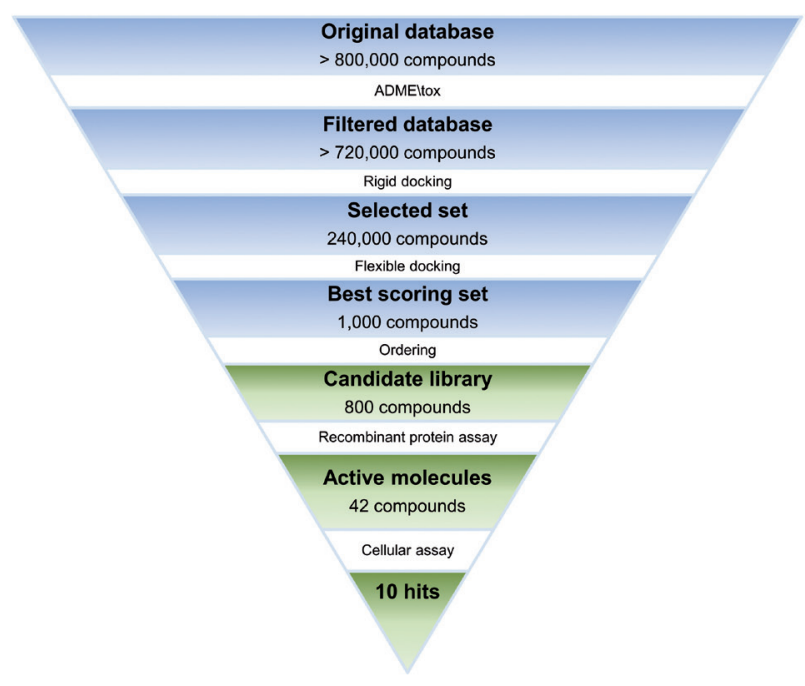

Figure 1. A screening coupling in silico and in vitro steps to identify glycogendelocalizing molecules

(A) 2D-alignment of both CBMs sequences (Homo sapiens, UniProt accession codes: Q9Y478.4 for $\beta 1$ and 043741.1 for $\beta 2$ ), with conservation represented in symbols (“*” full, ":" strong, and ".' weak conservation) and bar graph; numbering based on human $\beta 1$-sequence. Black arrows represent the known carbohydrate-binding residues, the blue arrow highlights the Thr101 insertion (in position 102 due to the numbering based on human $\beta 1$-sequence). (B) 3D-representations of AMPK $\beta 1$ - (left, PDB ID: 1Z0M) and $\beta 2$ - (right, PDB ID: 2F15) CBM in grey ribbon with the loop of positions 100-101 represented in blue and the binding pocket volume highlighted in orange balls. (C) Scheme representing the screening steps with associated number of compounds. The in silico steps are represented in blue and the in vitro ones in green; in white are the filtering steps. 
0.6 is acceptable for such pocket [48]. These eight protein conformations were thus selected for the virtual screening.

A commercially available database of small molecules was retrieved and screened first with the Lipinski rule of 5 to retrieve compounds which are more likely to possess drug-like properties: this resulted in exclusion of $\sim 10 \%$ of the compounds $(722,000$ compounds kept of the original 800,000 compounds; Fig. 1C). Subsequently, two consecutive steps of the virtual ligand screening of eight different CBM $\beta 2$ conformers followed an established protocol of rigid docking to remove the geometrically most unlikely compounds from the database, and then of a more exhaustive flexible docking that allows ligand and target side chain flexibility [31-33]. The docked poses of the 1,000 best scoring compounds were visually inspected and the top-800 were ordered for further testing in a two-step in vitro screening, as described in the following section (Fig. 1C). At the end of the process, 10 compounds were found positive in both assays, leading to a hit-rate of $1.25 \%$. Interestingly, six of the compounds clustered together when a cutoff of 0.5 was used in ChemMine Binning Clustering Tool, whereas each of the other four compounds was binned separately. Therefore, this cluster of compounds, which dominated the hits, is further described below.

\section{Identification of similar compounds with ability to dislocate the CBM}

The initial in vitro screening was based on a cell-free, carbohydrate-binding competition assay (Fig. 2A). First, the recombinant AMPK CBM $\beta 2$ domain was allowed to bind to the glycogen $\alpha 1-6$ turn analog, $\beta$-cyclodextrin, which was immobilized on a resin. The isolated CBM domain was chosen over the AMPK heterotrimer because its use increased the signal to noise ratio (Supp. Fig. 1A; 1.2 vs 3.8 using maltoheptaose for the heterotrimer and CBM respectively). Secondly, the compounds were added to the protein-resin complex in solution: since active compounds compete for interaction with the CBM, they should displace the protein from the resin-bound carbohydrate to the supernatant. The protein amount in the supernatant was estimated by the difference in absorbance, named "normalized sample absorbance", between signal measurements in the presence of a compound and of vehicle only (Fig. 2A). To alleviate a direct effect of compounds on the BCA protein determination, the BCA absorbance was measured with the compound alone, in the absence of protein, and subtracted with the absorbance of vehicle alone, to give the "normalized background absorbance".

The 800 compounds were first tested as mixes of four compounds at a concentration of $100 \mu \mathrm{M}$, and the mixes showing a significant difference between normalized background and sample absorbance were selected for individual testing. After initial screening of single compounds at a concentration of $100 \mu \mathrm{M}, 42$ single compounds were judged active (i.e. difference between background and sample absorbance of at 

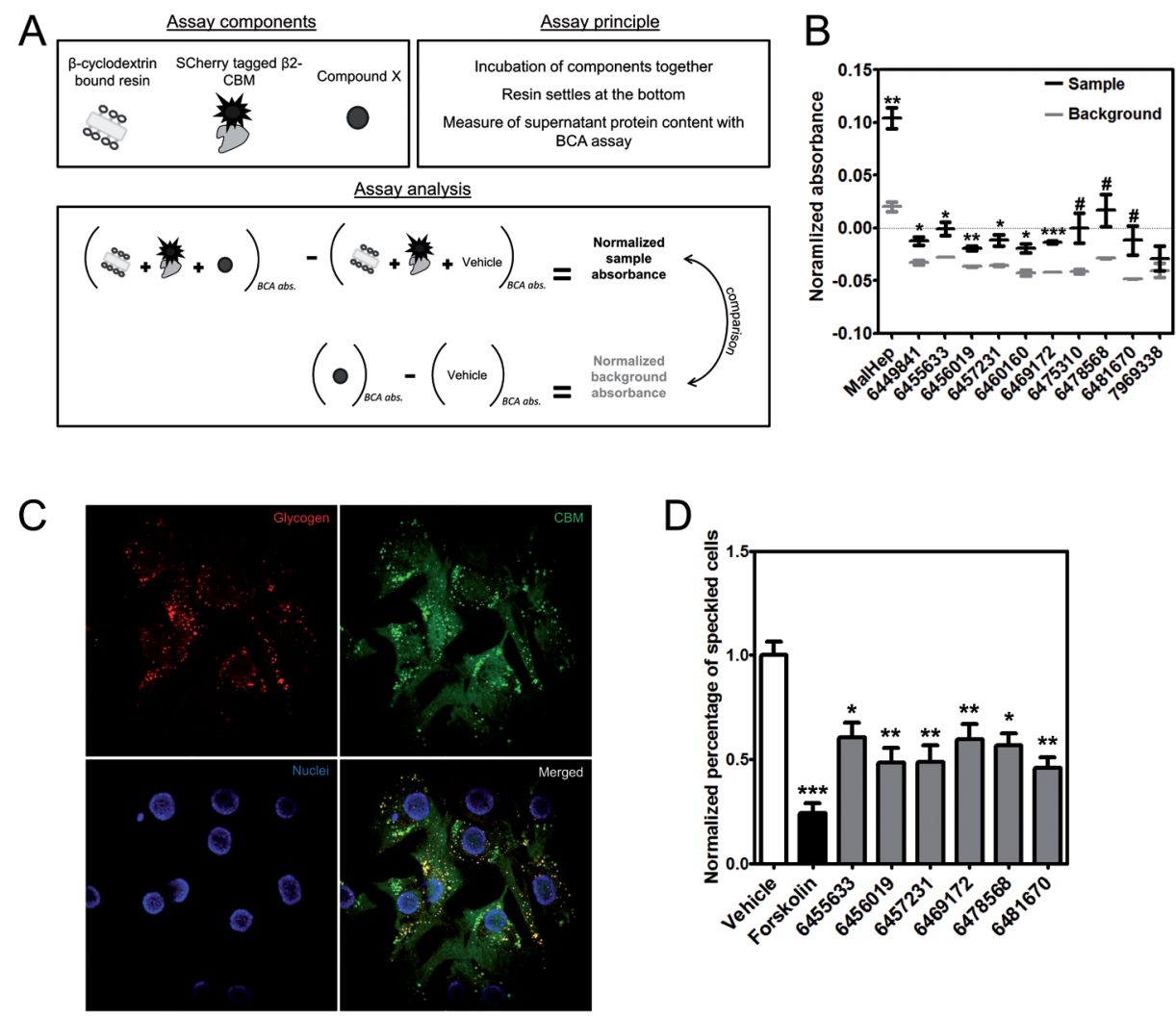

Figure 2. Identification of similar compounds with ability to dislocate the CBM

(A) Schematic representation of the carbohydrate-binding competition assay. (B) Normalized absorbances, as described in the scheme (background in grey, sample in black, represented as means with SEM), of the identified isoeugenol derivatives in the carbohydrate-binding competition assay. As described in the text, isoeugenol derivatives presumably interfered with the BCA readout, explaining the consistently negative normalized absorbances. Each compound and maltoheptaose (MalHep) were used at $100 \mu \mathrm{M}$. Statistical significance: Student's two-tailed $t$-test of sample vs. background absorbance, ${ }^{* * *}: \mathrm{p}<0.001,{ }^{* *}: \mathrm{p}<0.01$, $^{*}$ : $\mathrm{p}<0.05$, \# : $\mathrm{p}<0.1, \mathrm{n}=4$. (C) Representative image of C2C12 cells stably overexpressing the GFP-tagged CBM $\beta 2$ (in green). Cells were fixed then stained with an anti-glycogen antibody that was detected by an Alexa647-labeled secondary antibody (in red) and with DAPI for nuclei (in blue), after which co-localization was assessed by confocal imaging. (D) Percentage of cells showing the GFP speckled pattern normalized to level of vehicle ( $0.25 \%$ DMSO). Forskolin control was used at $100 \mu \mathrm{M}$ while the compounds were tested at $25 \mu \mathrm{M}$, in serumfree medium supplemented with $10 \mu \mathrm{g} / \mathrm{mL}$ digitonin. Statistical significance: Student's $t$-test compound vs. DMSO, *** : $\mathrm{p}<0.001,{ }^{* *}: \mathrm{p}<0.01,{ }^{*}: \mathrm{p}<0.05, \mathrm{n}=4$.

least 0.01 ), with a significant difference in 30 cases and near-significant difference in 12. Interestingly, nine of these compounds showed a very similar structure with two benzene rings linked by a flexible chain of 6-7 atoms of carbon and oxygen (Table 1). 
The ring " $A$ " shared the exact same features across all hit compounds while the substitutions on ring "B" vary. Since the substitutions of the ring "A" correspond to those of the natural product isoeugenol, this group of nine compounds is referred to as isoeugenol derivatives in the rest of the text. Isoeugenol derivatives exhibited a lower background absorbance compared to the vehicle (negative normalized background absorbance) probably by either stabilizing the copper of the BCA assay in an oxidized state or influencing directly the absorbance measurement. Six of them showed a significant difference with the normalized sample absorbance, and the three others reached near-significance (Fig. 2B), and were therefore considered active in this assay and included in the subsequent screening step. Of the complete set of tested compounds, only one other compound shared the isoeugenol feature of the common structure (7969338, Table 1), however this compound showed no activity in the carbohydratebinding competition assay (Fig. 2B). The shorter linker and bulkier ring "B" found in this compound compared to the active isoeugenol derivatives point toward a structureactivity relationship.

For the following screening step, we used C2C12 cells stably over-expressing the GFP-tagged CBM $\beta 2$, which is known to show a punctuate pattern of GFP-positive areas that co-localize with glycogen (Fig. 2C) [15]. Cells were incubated in the absence or presence of compounds and were monitored for the (dis)appearance of this peculiar pattern upon treatment. As a proof-of-concept, use of forskolin, an agent that depletes glycogen through the cAMP pathway, led to an $80 \%$ reduction in speckle amount (Fig. 2D). For the initial screening process, a small amount of digitonin was added to the serum-free medium, which allowed a better crossing of the membrane (Supp. Fig. 1B) without showing any obvious cytotoxicity. In this experimental setting, three of the nine aforementioned compounds were unfortunately not sufficiently soluble for testing, probably due to the relatively high hydrophobicity of this class of compounds $(\log \mathrm{P} \approx 5)$. In contrast, all other six compounds were found to significantly reduce the incidence of speckled pattern of the GFP-tagged CBM $\beta 2$, as judged by the observed lowering in the percentage of speckled cells compared to vehicle (Fig. 2D). Since they all decreased the number of speckles by more than the arbitrary threshold of $35 \%$, the six compounds were judged active in cells and therefore classified as hit molecules. In summary, our two-step screening process following the virtual ligand screening, allowed us to identify isoeugenol derivatives with effects on carbohydrate binding both in a cell-free in vitro assay and in a cellular assay on subcellular localization.

Two of the identified compounds have a micromolar activity and directly bind the $C B M$

To follow up on the cellular activity of the 6 identified compounds, half maximal 


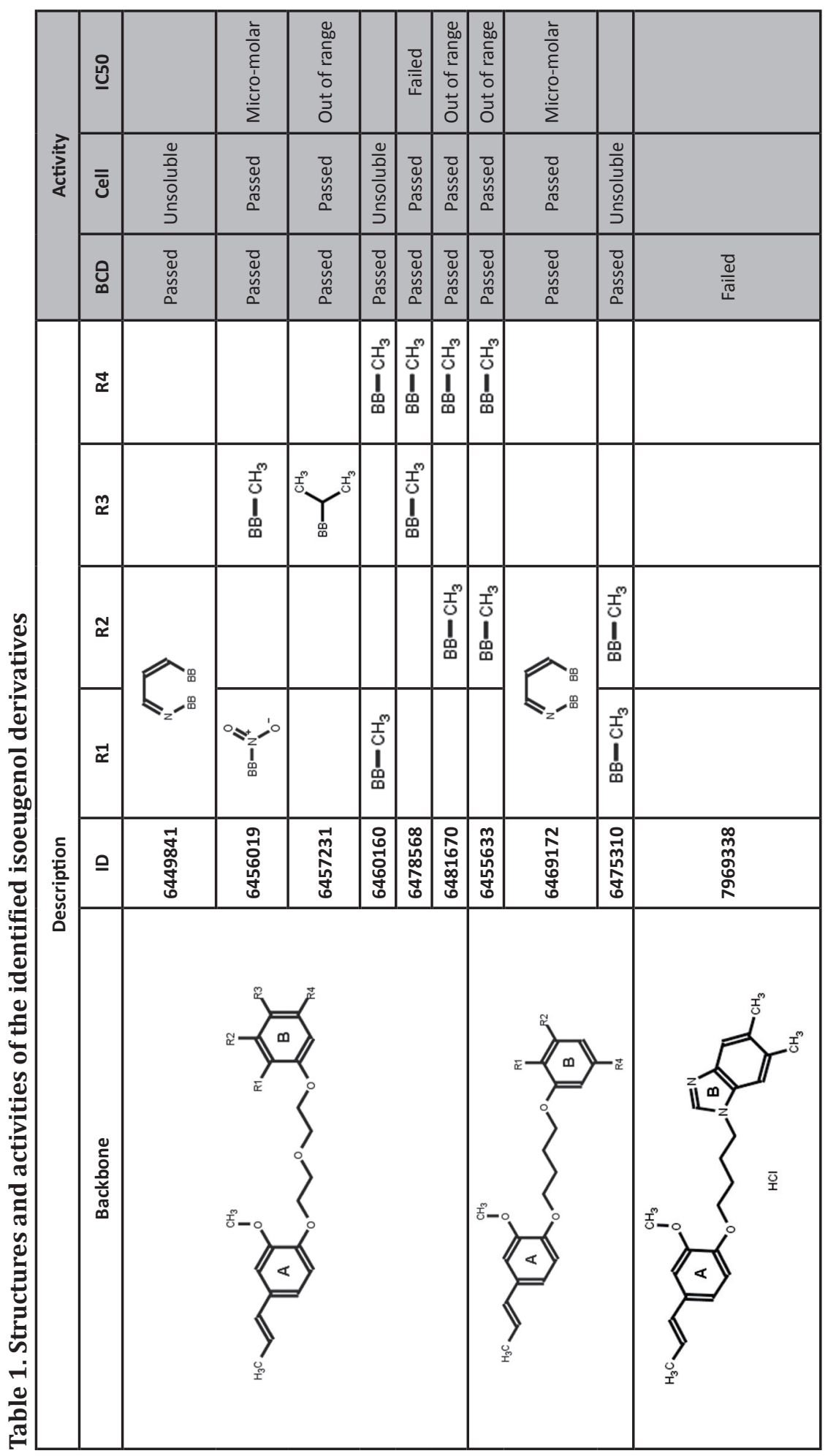

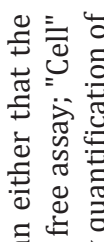

ฮี

पे

范

出

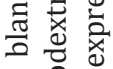

온 을

تै

त्रे Ф્.

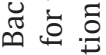

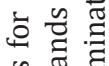

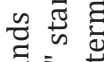

荧守

=

ص

$\therefore$ 일

․ㅜㅇㅇㅛ

要考离

든

ํㅡㄹ

¿ : : 근

임 䒕

웜듀

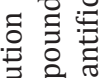

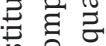

给论

Ð $\frac{\pi}{ \pm}$

온돈

든

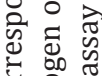

이유.ㄴ

要寻

T 0

สี

๙

तै ${ }^{2}$ के के

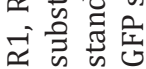


inhibitory concentrations (IC50) were measured in absence of digitonin and in presence of serum by testing different concentrations in the cell-based assay. The compounds 6456019 and 6469172 showed micromolar activity (1.3 [0.1-14] and 8.9 [4-19] $\mu \mathrm{M}$ [95\% confidence interval, CI95] respectively; Fig. 3A). The compound 6456019 only displaced up to $49 \%$ of the total amount of speckles, while addition of compound 6469172 maximally resulted in a 92\% displacement. However, the compounds 6455633,6457231 and 6481670 seem to have little activity at the described concentrations and their IC50 could not be determined. The compound 6478568 did not show a significant decrease in speckle amount at any of the concentrations. This lack of activity of the four latter compounds is inconsistent with the effects seen during the cellular screening, which might for example be explained by their inability to cross the membrane readily in the absence of digitonin.

For the two remaining hit molecules with low micromolar activity (6469172 and 6456019), surface plasmon resonance was performed on the immobilized CBM $\beta 2$ to assess direct interaction. The resulting dose-dependent response confirmed direct binding. Supporting a direct effect in the cellular assay, the estimated dissociation constants of both compounds were in the same range as their IC50, with overlapping 95\% confidence intervals (5.4 [3.9-7.5] and 25 [19-33] $\mu \mathrm{M}$ [CI95] for 6456019 and 6469172 respectively; Fig. 3B). Therefore, the compounds 6456019 and 6469172 show high activity towards speckle disappearance in cells even in the absence of digitonin, and can directly bind to the recombinant CBM $\beta 2$. To further confirm a potential role of the compounds in glycogen-related pathways and to test the effect on heterotrimeric AMPK, we investigated the effect of the compounds 6469172 and 6456019 on the interaction of AMPK with glycogen-bound downstream targets in a cell-free setting. The heterotrimer AMPK $\alpha 1 \beta 2 \gamma 1$ was immunoprecipitated from cotransfected HEK293T cells (myc- $\alpha 1, \beta 2, \gamma 1$ ). Under basal condition, glycogen synthase immunoprecipitated with AMPK (Fig. 3C). However, when the cell lysate was preincubated with the glycogen mimetic, $\beta$-cyclodextrin, glycogen synthase was no longer recovered in the immunoprecipitate of AMPK, suggesting that glycogen may act as a scaffold between the two proteins in the lysate and that $\beta$-cyclodextrin disrupts their glycogen binding. Both compounds 6469172 and 6456019 similarly decreased the interaction of AMPK with glycogen synthase, showing their ability to mimic $\beta$-cyclodextrin and therefore suggesting a common binding pocket and action on AMPK. To verify that this effect was not an artifact, the same experiment was carried out with a CBM-unrelated AMPK activator, oligomycin (Supp. Fig. 2). As expected, oligomycin did not disrupt the co-immunoprecipitation of AMPK and glycogen synthase. Therefore, the effect of 6469172 and 6456019 on AMPK heterotrimer was specific and similar to that induced by $\beta$-cyclodextrin. 
A

\begin{tabular}{|c|c|c|}
\hline ID & $\begin{array}{c}\text { IC50 in } \mu \mathrm{M} \\
\text { (CI95) }\end{array}$ & $\begin{array}{c}\text { Max. } \\
\text { inhibition } \\
\text { (Stdev) }\end{array}$ \\
\hline 6456019 & $1,3(0-14)$ & $49 \%(11)$ \\
\hline 6469172 & $8,9(4-19)$ & $92 \%(7)$ \\
\hline 6455633 & \multicolumn{2}{|c|}{ Out of range } \\
\hline 6457231 & \multicolumn{2}{|c|}{ Out of range } \\
\hline 6481670 & \multicolumn{2}{|c|}{ Out of range } \\
\hline 6478568 & \multicolumn{2}{|c|}{ ND } \\
\hline
\end{tabular}

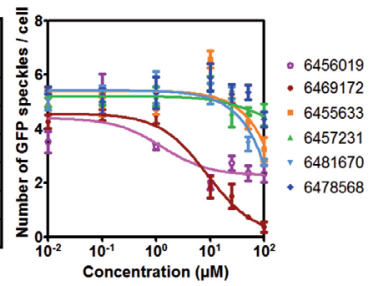

C

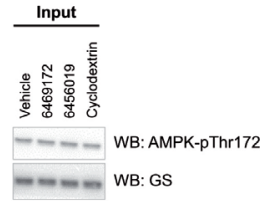

$\mathrm{B}$

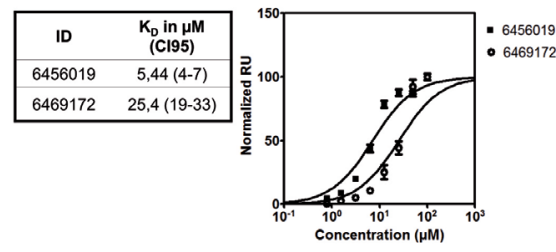

D

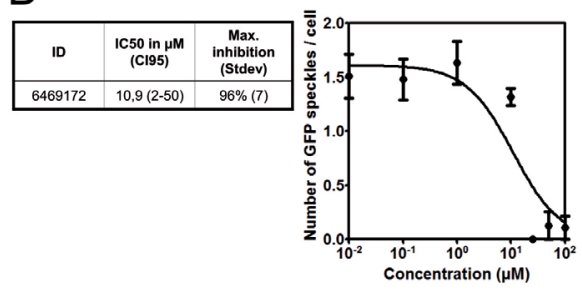

Figure 3. Two of the identified compounds have a micromolar activity and directly bind the CBM

(A, D) IC50 determination based on the average number of speckles per cell following treatment with a range of concentration $(0-100 \mu \mathrm{M})$. Assay was carried out for a selected number of compounds with CBM 32 -expressing C2C12 cells (A) and for 6469172 with CBM 31 -expressing C2C12 cells (D). Max inhibition refers to the maximal percentage of speckles removed by the tested compound. CI95: 95\% confidence interval, Stdev: standard deviation, $n=3$ repeats with $>150$ imaged cells each. (B) Determination of the equilibrium dissociation constant $\left(\mathrm{K}_{\mathrm{D}}\right)$ by surface plasmon resonance using immobilized CBM $\beta 2$ and different concentrations of 6456019 and $6469172(0-50 \mu \mathrm{M}$ for 6456019 and $0-100 \mu \mathrm{M}$ for 6469172); $n=4$. (C) Lysate of AMPK-overexpressing mammalian cells treated with either vehicle $(0.5 \%$ DMSO), $25 \mu \mathrm{M}$ of compound 6469172 or 6456019 , or $5 \mathrm{mM} \beta$-cyclodextrin (left panel). Immunoprecipitation of AMPK heterotrimer was performed with the myc antibody following the treatment (right panel). The used antibodies recognize total level of glycogen synthase (GS), total level of AMPK- $\beta 2$ subunit (AMPK- $\beta 2$ ), and phosphorylation status of AMPK at Thr172 (AMPK-pThr172); $n=2$

After confirming an effect of both compounds on the CBM $\beta 2$ and on the $\beta 2$-containing AMPK heterotrimer, we sought to check for isoform specificity. In view of possessing both micromolar IC50 and high amplitude of action, compound 6469172 was selected for further testing on cells expressing the GFP-tagged CBM $\beta 1$ in the same setting as the described cellular screening. Expectedly, owing to the lack of pocket specificity, the determined IC50 was similar for both protein isoforms (10.9 [2-50] $\mu \mathrm{M}$ for CBM $\beta 1$ vs. previously mentioned 8.9 [4-19] $\mu \mathrm{M}$ for CBM $\beta 2$ [CI95]; Fig. 3A and 3D), suggesting absence of $\beta$-isoform selectivity.

In summary, the compounds 6469172 and 6456019 are direct binders of the carbohydrate-binding module of AMPK and have an activity in the micromolar range to change the subcellular localization of AMPK away from glycogen. 
Docking poses of the identified compounds suggest an explanation for the difference in affinity and absence of isoform-specificity of the identified compounds

The six compounds of the identified cluster tested for IC50 (Table 1) were re-docked into the binding pocket of $\mathrm{CBM} \beta 2$ structures with AutoDock Vina to obtain a more detailed insight into their structure-activity relationship. Molecular docking of the active compounds (6456019 and 6469172) yielded two different possible binding modes for each compound. All binding poses share a similar orientation of the common ring " $\mathrm{A}$ " toward the loop of Thr101 (numbering of the human sequence of AMPK- $\beta 2$ ), while the linker is positioned between Trp99 and the loop of Leu146 and the variable ring "B" is close to Gln124 (Fig. 4A and 4B, Supp. Fig. 3A and 3B). While in all conformations, the compounds occupy the carbohydrate-binding site (as seen in the right panel of Fig. 4A compared with pocket definition shown in the right panel of Fig. 1B), the orientation of the rings account for main differences between the two poses found for each compound.

Several binding free energy calculations (e.g. MM/PB(GB)SA and QM/MM-GBSA), which were previously shown to perform well in predicting binding affinity $[42,49]$ and binding mode $[43,50,51]$, were applied to estimate the binding strength for the two compounds in these two different poses. For both compounds 6456019 and 6469172, the poses depicted in Fig. 4A and 4B (defined as mode 1) had consistently lower binding free energy across calculation methods, compared to those in Supp. Fig. 3A and 3B (defined as mode 2) (Supp. Fig. 3C). Therefore, the binding mode 1 is more favorable and represents a likely binding mode of these analogous compounds in the binding pocket of CBM $\beta 2$.

Interestingly, the position occupied by the compound in the proposed binding modes resides in an area that is identical between CBM $\beta 1$ and CBM $\beta 2$ (Fig. 4C). Although the ring " $\mathrm{A}$ " substitution points towards the loop formed by Thr101 in the $\beta 2$-isoform, the compound does notfully occupy the extra space and can therefore also be accommodated in the smaller pocket of the $\beta 1$-isoform. Besides, the Thr101 residue, which is not present in CBM $\beta 1$, is flipped towards the outside of the binding pocket and cannot form additional interactions. Thus, these data could explain the absence of isoformspecificity seen in the in vitro experiments (Fig. 3D). When looking at the poses of the two most active compounds, the linker of 6456019 seems more interesting than that of 6469172 since the extra oxygens can form hydrogen-bonds with Trp99 and Asn150 (Fig. 4B). Interestingly, substitutions to the ring B of both compounds interact with additional residues (Fig. 4A and 4B): the nitro group at the R1 of 6456019 induces an electrostatic interaction with Asn98, while in R1/R2 of 6469172, the pyridine ring forms a hydrogen bond with Trp99. In contrast, none of the four other compounds 

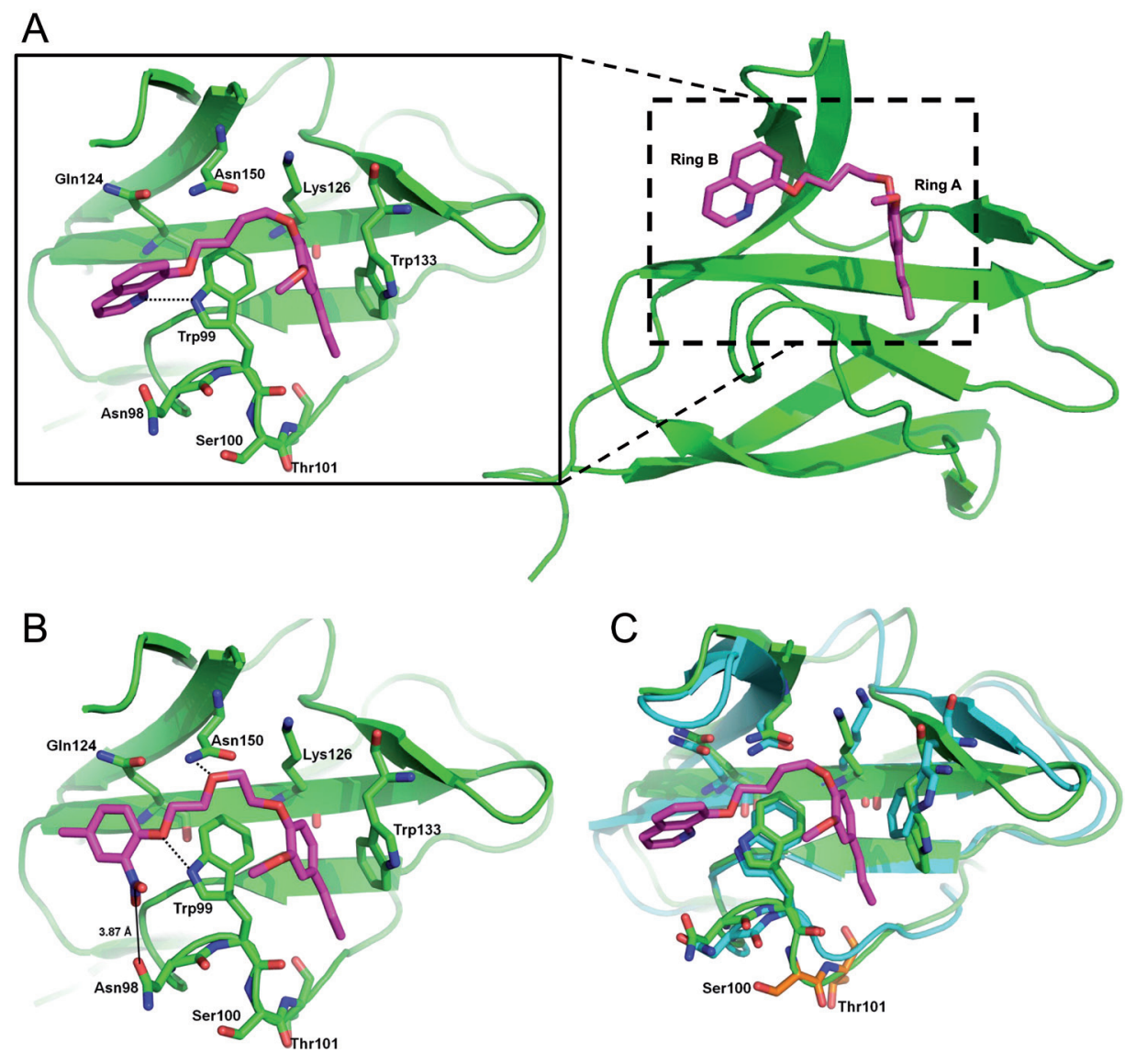

Figure 4. Docking poses of the identified compounds suggest an explanation for the difference in affinity and absence of isoform-specificity of the identified compounds (A-B) Predicted binding modes (defined as mode 1) of 6469172 (A) and 64556019 (B) in CBM $\beta 2$ carbohydrate-binding pocket. The overall fit of the compound 6469172 is illustrated in the whole domain of CBM $\beta 2$ represented in green ribbon (A, right panel), whereas the other representations offer a closer view of the binding modes with explicit residues shown in sticks. Hydrogen bonds are shown as dashed lines. For $6469172, \pi-\pi$ interaction between the benzene ring and Trp133 is observed. For 6456019, the distance between nitrogen of the $\mathrm{NO}_{2}$ group of the compound and oxygen of the side chain of Asn98 is $3.87 \AA$, which can induce electrostatic interaction. (C) Docking pose of the compound 6469172 on superimposed CBM $\beta 1$ (cyan ribbon and stick) and CBM 32 receptors (green ribbon and stick, with the insertion loop Ser100-Thr101 shown in orange stick).

tested for IC50 (6457231, 6478568, 641870 and 6455633) could form these stabilizing interactions since their substitutions on ring B are only hydrophobic groups (Table 1). Therefore, the extra hydrogen bonds with Trp99 and Asn98 formed by 6469172 and 6456019 could explain the difference seen in IC50.

Among the different methods used to calculate binding free energy (Supp. Fig. 3C), 
the QM/MM-GBSA approach using PM6-DH+ method shows the best performance since the calculated binding free energy $\left(\Delta G_{\text {cal. }}\right)$ of the 6456019 and 6469172 compound derived by this method is in close agreement with the available experimental data $\left(\Delta G_{\text {exp. }}\right)$. The free energy differences of the 6456019 compound are $\Delta G_{\text {cal. }}-7.93 \mathrm{kcal} / \mathrm{mol}$ vs. $\Delta G_{\text {exp. }}-7.23 \mathrm{kcal} / \mathrm{mol}$ and for $6469172 \Delta G_{\text {cal. }}-6.57 \mathrm{kcal} / \mathrm{mol} \mathrm{vs.} \Delta G_{\text {exp }}-6.93 \mathrm{kcal} / \mathrm{mol}$. Thus, this binding free energy calculation method was also applied to estimate binding affinity of the other 4 selected compounds. The inactive compounds (6457231, 6478568,641870 and 6455633 ) gave positive or less negative binding free energy difference implying an unfavorable binding to $\mathrm{CBM} \beta 2$, which is in agreement with the experimental data (Table 2).

In summary, the re-docking data offer interesting explanations for the lack of isoform specificity and for differences in binding affinities between compounds. The agreement between virtual and experimental data support the suggested binding modes and thus suggests a direct binding in the carbohydrate-binding pocket of AMPK.

\section{Compound 6469172 is a cellular but not an allosteric activator of AMPK}

Compound 6469172, which showed the highest activity in the cell-based assay to measure subcellular localization (Fig. 3A), was next studied for its ability to influence the AMPK pathway in C2C12 cells. Dose-dependent activation of AMPK and phosphorylation of its downstream target acetyl-CoA carboxylase (ACC) were seen after compound treatment (Fig. 5A). At higher concentrations, the effect of compound 6469172 was similar to that of oligomycin, an indirect AMPK activator. This AMPK activating effect was not due to cytotoxicity of the compound, as appeared from the MTT assay results where no difference in cell viability was seen at any concentration compared to vehicle (Supp. Fig. 4). Activation by 6469172 was also time-dependent with a quick onset of AMPK and ACC phosphorylation but reaching a maximal activation after a longer period of time (Fig. 5B). In contrast, the oligomycin effect hit a maximal action on phosphorylation quickly and then faded away, suggesting either a quicker absorption or a different mechanism of action for these two AMPK activators.

Based on the previous results (Fig. 3 and 4), we did not expect $\beta$-isoform specificity, which we sought to confirm using the near-haploid cell line HAP1. Analysis of HAP1 cell lysates showed clearly increased AMPK and ACC phosphorylation upon 6469172 treatment and a milder response for AICAR (Fig. 5C). Interestingly, A-769662 treatment led to an increase in ACC phosphorylation while AMPK phosphorylation remained low, suggesting that AMPK allosteric activation drives most of the effect in this cell line. In HAP1 cell lines, either of the two $\beta$-subunit isoforms were targeted using the CRISPR/ Cas9 system resulting in clean knock-outs as can be seen from the $\beta$-subunit expression (Fig. 5C). As was expected, upon treatment with 6469172 both knockout cell lines 
Table 2. Experimental and predicted binding free energy of the compounds tested for IC50 determination.

\begin{tabular}{|c|c|c|c|c|}
\hline Compound ID & $K_{D}(\mu M)$ & $\Delta G_{\text {exp. }}(\mathrm{kcal} / \mathrm{mol})$ & $\begin{array}{c}\Delta G_{c a l .} \\
(\mathrm{QM} / \mathrm{MM}-\mathrm{GBSA}, \mathrm{PM6}-\mathrm{DH}+)\end{array}$ & $\left|\Delta G_{\text {exp. }}-\Delta G_{\text {cal. }}\right|$ \\
\hline 6481670 & & & 1.89 & \\
\hline 6455633 & & & 4.98 & \\
\hline 6456019 & 5.44 & -7.23 & -7.93 & 0.70 \\
\hline 6457231 & & & -1.19 & \\
\hline 6478568 & & & -1.67 & \\
\hline 6469172 & 25.40 & -6.31 & -6.57 & 0.26 \\
\hline
\end{tabular}

showed strong Thr-172 phosphorylation signals comparable to the WT, confirming the absence of $\beta$-isoform specificity. Similarly, AICAR activated AMPK in all cell lines, although somewhat stronger Thr-172 phosphorylation signals were obtained for the $\beta 1$-knockout. A-769662 treatments were also comparably efficient in knockout cell lines and WT with regard to enhancement of ACC phosphorylation signal. In contrast to the WT and $\beta 2$-knockout cell lines, treatment with A-769662 increased AMPK Thr-172 phosphorylation in the $\beta 1$-knockout cell line, indicating that in absence of the $\beta 1$ isoform, the action of A-769662 on AMPK may follow another mechanism (i.e. change in phosphorylation status rather than allosteric activation; see Discussion for details). Therefore, the 6469172 compound activates both $\beta 1$ - and $\beta 2$ - containing AMPK heterotrimers.

AMPK is mainly activated through three methods: allosteric activation, facilitation of Thr172 phosphorylation or protection from Thr172 dephosphorylation. To test whether 6469172 acted allosterically to upregulate AMPK activity, immunoprecipitated myc-tagged AMPK heterotrimers from triple-transfected HEK293T cells (myc- $\alpha 1, \beta 2$, $\gamma 1)$ were tested for kinase activity. The enzymatic activity assessed by this assay seemed truly specific to AMPK, since the addition of SAMS peptide (substrate for AMPK) led to a dramatic increase in ATP consumption (Fig. 5D). The presence of the known allosteric activator A-769662 increased basal AMPK activity of the $\alpha 1 \beta 1 \gamma 1$ but not $\alpha 1 \beta 2 \gamma 1$ complex. On the contrary, and as expected, the compound 6469172 failed to increase AMPK activity in both cases, suggesting that 6469172-induced AMPK activation in cells may rather depend on increased Thr172 phosphorylation than on a direct allosteric effect. Therefore, we conclude that 6469172 is a novel AMPK activator without $\beta$-isoform specificity, whose mechanism of action does not rely on allosteric effect. 

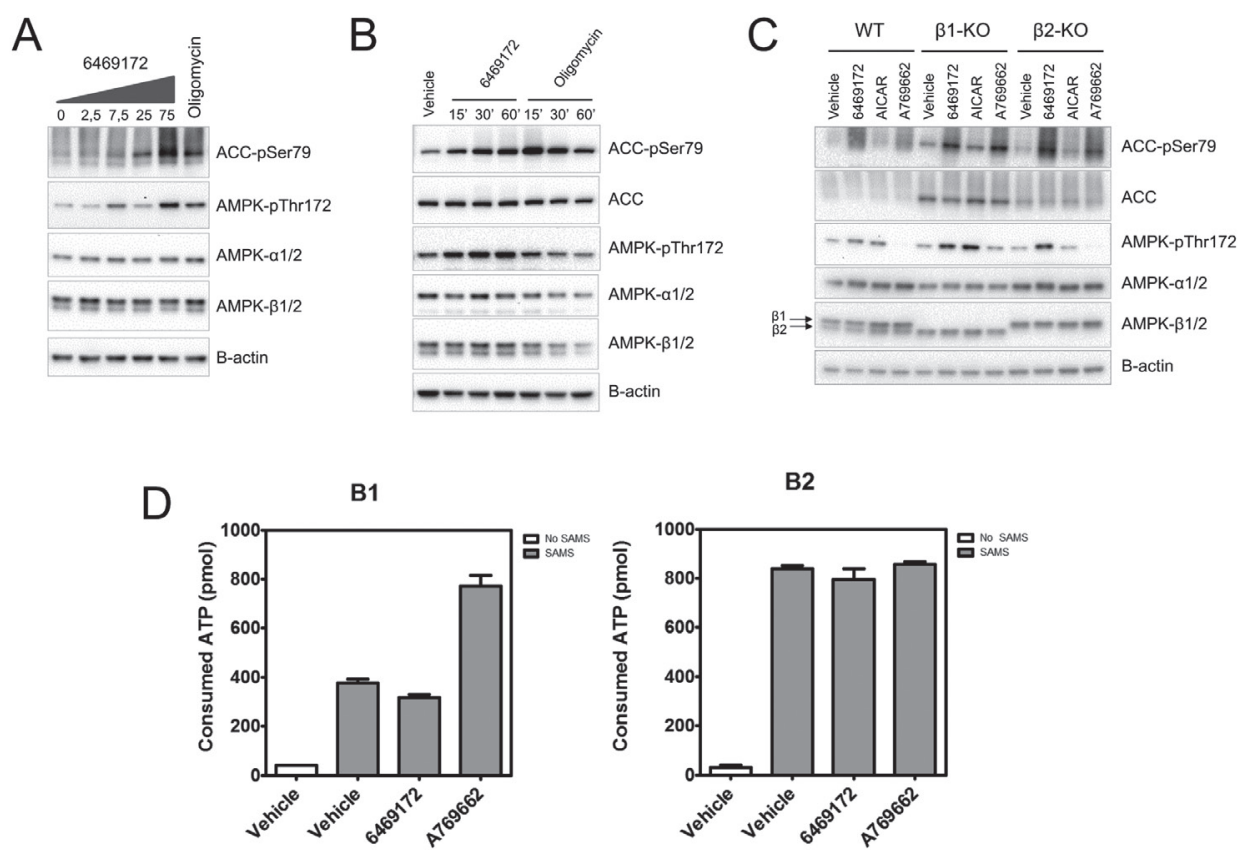

Figure 5. Compound 6469172 is a cellular but not an allosteric activator of AMPK

(A) Dose-dependent response of 6469172 treatment on AMPK and ACC phosphorylation. Assay carried out in C2C12 cells after 1.5 hour-treatment, doses in $\mu \mathrm{M}$ with a constant amount of vehicle (1\% DMSO). Oligomycin was used at $5 \mu \mathrm{M}$ for 1.5 hour. (B) Time-dependent response of AMPK and ACC phosphorylation in C2C12 cells after 15, 30 or 60-minute treatment with $25 \mu \mathrm{M}$ of 6469172 compound or $5 \mu \mathrm{M}$ oligomycin with a constant amount of vehicle (1\% DMSO, 60 minute-treatment). (C) Treatment of the haploid cell line HAP1, in its WT form, or with a knock-out of either the AMPK- $\beta 1$ ( $\beta 1$-KO) or - $\beta 2$ subunit ( $\beta 2$-KO). 2-hour treatment of vehicle ( $0.25 \%$ DMSO) or $50 \mu \mathrm{M} 6469172$, or 1 -hour treatment of $1.5 \mathrm{mM}$ AICAR or $100 \mu \mathrm{M}$ A769662. (A-C) The used antibodies recognize total level of ACC protein (ACC), phosphorylation status of ACC at Ser79 (ACC-pSer79), total level of AMPK- $\alpha$ subunit (AMPK- $\alpha 1 / 2)$, total level of AMPK- $\beta$ subunit (AMPK- $\beta 1 / 2$ ), phosphorylation status of AMPK at Thr172 (AMPK-pThr172) and total level of $\beta$-actin (B-actin) which was used as loading control. (D) Kinase activity assay on myc-tagged AMPK heterotrimers $\alpha 1 \beta 1 \gamma 1$ (left panel) or $\alpha 1 \beta 2 \gamma 1$ (right) immunoprecipitated from mammalian cells. Assays were done in the absence (white bars) or presence (grey bars) of $100 \mu \mathrm{M}$ SAMS peptide, an AMPK substrate, and with $100 \mu \mathrm{M} 6469172$ or $50 \mu \mathrm{M}$ A769662 in constant vehicle content (5\% DMSO).

\section{The identified compound affects metabolism-related cell lines and pathways}

Muscle and liver are important organs for glycogen storage and therefore are of particular interest for our study. To assess the ability of 6469172 to activate AMPK in more physiologically relevant cell types, C2C12 cells were differentiated into myotubes. The treatment with 6469172 induced a strong AMPK and ACC phosphorylation comparable to that of other known AMPK activators (Fig. 6A). Secondly, the human 


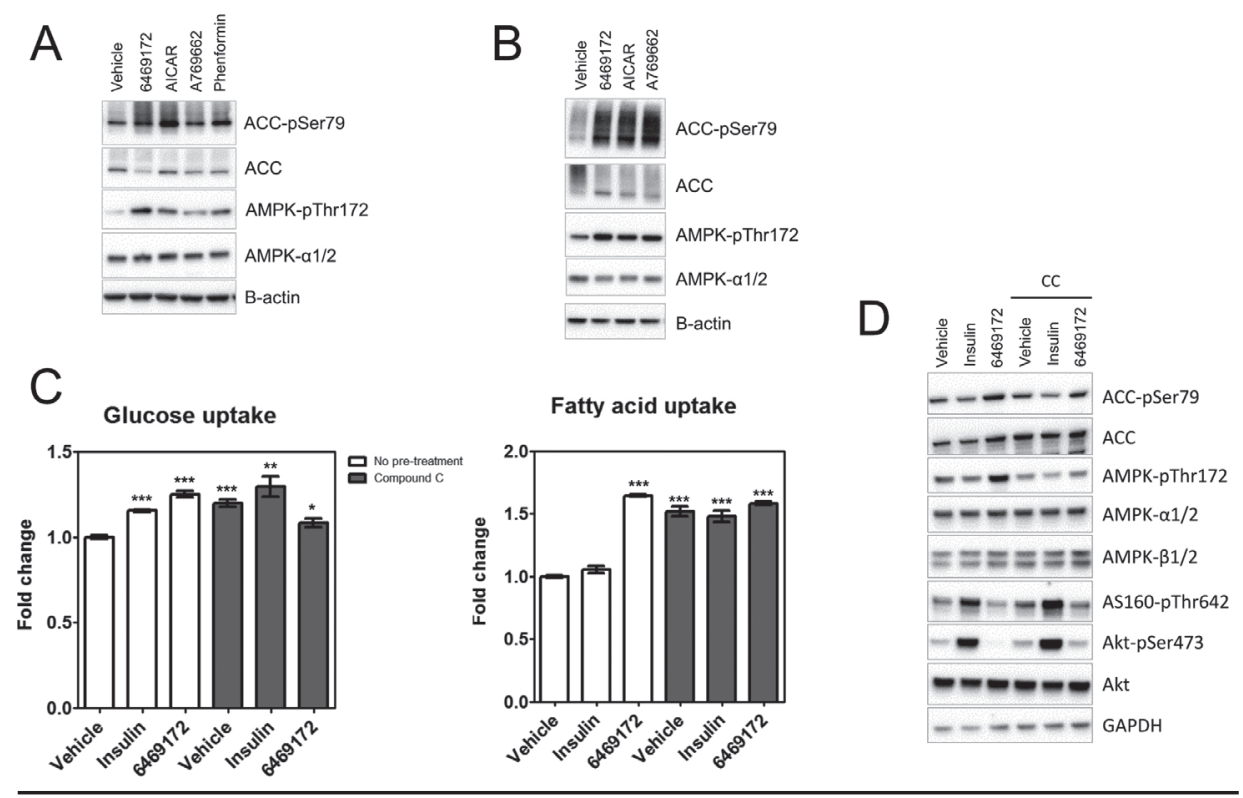

Figure 6 . The identified compound affects metabolism-related cell lines and pathway

(A) Treatment of $\mathrm{C} 2 \mathrm{C} 12$ cells differentiated in myotubes: 2-hour treatment with vehicle (1\% DMSO) or $75 \mu \mathrm{M}$ 6469172, or 1-hour treatment with 1.5 mM AICAR, $100 \mu \mathrm{M}$ A769662 or 1.5 mM phenformin. (B) Treatment of the hepatic cell line HepG2: 2-hour treatment with vehicle

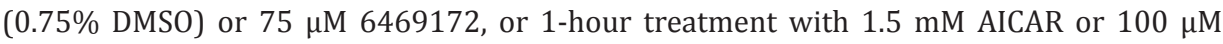
A769662. (C) Glucose uptake measurement following treatment with vehicle (0.5\% DMSO), $100 \mathrm{nM}$ insulin or $25 \mu \mathrm{M}$ 6469172, with (grey bars) or without (white bars) pre-treatment with $10 \mu \mathrm{M}$ Compound C. Statistical significance: Student's $t$-test of vehicle without Compound C vs other treatment, ${ }^{* * *}: \mathrm{p}<0.001,{ }^{* *}: \mathrm{p}<0.01,{ }^{*}: \mathrm{p}<0.05, \mathrm{n}=4$. (D) Western Blot resulting from the previously described glucose uptake experiment. $\mathrm{CC}=$ Compound $\mathrm{C}$ pretreatment. (A-B, D) The used antibodies recognize total level of ACC protein (ACC), phosphorylation status of ACC at Ser79 (ACC-pSer79), total level of AMPK- $\alpha$ subunit (AMPK- $\alpha 1 / 2$ ), total level of AMPK- $\beta$ subunit (AMPK- $\beta 1 / 2$ ), phosphorylation status of AMPK at Thr172 (AMPK-pThr172), phosphorylation status of AS160 at Thr642 (AS160-pThr642), total level of Akt protein (Akt), phosphorylation status of Akt at Ser473 (Akt-pSer473) and total level of $\beta$-actin (B-actin) which was used as loading control.

hepatoma cell line HepG2 was also sensitive to 6469172 and other AMPK activator treatments, showing a strong AMPK and ACC phosphorylation (Fig. 6B). Therefore, the compound 6469172, which was shown to have the most interesting effect on CBM displacement from glycogen, also activates the AMPK pathway in relevant cell lines.

A well-documented role of AMPK is the increase of glucose and fatty acid uptake by triggering transporter translocation to the membrane [6]. In HL1 cardiomyocytes, treatment with 6469172 resulted in an increase in glucose uptake similar to that induced by insulin (Fig. 6C, left panel). Surprisingly, pre-treatment with the AMPK inhibitor Compound $\mathrm{C}$ raised the unstimulated levels of glucose uptake. A trend toward 
a further increase was seen upon insulin co-treatment, whereas the compound 6469172 in conjunction with Compound C did not lead to any further increase. Regarding fatty acid entry into HL1 cardiomyocytes, the same increase in unstimulated uptake was seen with Compound C (Fig. 6C, right panel). With or without Compound $\mathrm{C}$, insulin failed to trigger an increase in fatty acid uptake in that cell line. On the contrary, the compound 6469172 led to a 1.6-fold increase compared to basal, which was blunted after Compound $\mathrm{C}$ treatment. These results were mirrored by the Western Blot results (Fig. 6D): insulin strongly upregulated Akt and AS160 phosphorylation and slightly decreased AMPK and ACC phosphorylation, whereas 6469172 had the exact opposite effect on both pathways. Compound $C$ reduced the AMPK and ACC phosphorylation even in presence of 6469172 and triggered the insulin pathway, as seen with the increased Akt and AS160 phosphorylation in each treatment, which could explain the increased nutrient uptake in Compound $C$ treated samples. These results point to a potential beneficial effect of treatment with 6469172, since showing increased glucose and fatty acid uptake in an insulin-independent manner.

\section{Discussion}

In this study, we first established a new protocol to target the CBM of AMPK by means of virtual ligand screening followed by a carbohydrate binding competition assay and monitoring of CBM subcellular localization. Using this workflow we identified a set of structurally related compounds, the isoeugenol derivatives, with satisfactory scores in all screening steps. Two of these were found to bind directly to the CBM by surface plasmon resonance and mimic the $\beta$-cyclodextrin induced dissociation of the AMPK heterotrimer from another glycogen-bound protein, i.e. glycogen synthase. The differences in activity seen in the cluster of isoeugenol derivatives (Table 1) could be explained by the presence of additional hydrogen-bonds in the most active ones that are predicted to stabilize the interaction between the compound and the CBM. The compound 6469172 additionally potently activated AMPK in several relevant cell lines, but did not allosterically activate AMPK as determined by cell-free kinase assays. Furthermore, 6469172 triggered increased nutrient uptake in HL1 cardiomyocytes that was correlated to AMPK activation and blunted by treatment with the AMPK inhibitor Compound C.

The method of screening we established here is meant to identify agents that disrupt the glycogen-AMPK interaction. The in silico screen coupled with a two-step in vitro assessment led to a hit rate of $1.25 \%$, which is in the reported range for a typical virtual screening (1-5\%) and better than that of classical high-throughput screenings $(\leq 0.01 \%$ success) [52]. Higher success rates are generally achieved in virtual screenings when the amount of data on receptor structure and known ligands is high [52]. In our case, 
only carbohydrates were known to bind to the CBM at the start of the project and they cannot be used for training in a classical virtual screening since requiring specific docking methods [53]. The identification of a first set of active and inactive compounds, and the production of direct-binding experimental data, is therefore a great opportunity for further in silico screening attempts. In particular in the phase of compound development and optimization, computer-based techniques help to prioritize the possible compounds to be tested [54]. In this study, the binding free energy calculations support the proposed binding modes since the results of the QM/MM-GBSA approach on these poses are in conformity with the experimentally determined values. However, in other studies, this method has also been successfully applied prior to compound ordering to further discriminate potential active and inactive compounds [43]. Given the promising results obtained in this application, we expect such calculations to be useful for further lead generation of the isoeugenol derivatives as well.

The absence of $\beta$-isoform specificity for the identified compounds could be foreseen by the high global sequence similarity between the two isoforms and especially within the binding pocket (Fig. 1A). However, the predicted binding poses found by re-docking of the active compounds, which are supported by binding free energy calculations, show that the identified compounds do not fully take advantage of the larger pocket induced by the Thr101 insertion in the $\beta 2$-isoform (Fig. 4A). Hence, it identifies a strategy for further hit-to-lead optimization to possibly reach isoform specificity. Nevertheless, isoform preference rather than specificity may be a more appropriate goal in the AMPK field, considering the close resemblance between AMPK holoenzymes and their complex regulation. For instance, the well-characterized activator A-769662 was often reported as being $\beta 1$-isoform specific: in recombinant protein activity assays, only $\beta 1$-containing complexes are activated independently of the $\alpha$ and $\gamma$-isoforms [55], while in cells and tissues expressing mainly $\beta 2$ [56] as well as in $\beta 1$ knockouts [57], A-769662 fails to activate AMPK and AMPK-dependent pathways. In agreement with this view, in the kinase activity assay of this study, the immunoprecipitated $\beta 1$ containing complex, but not the $\beta 2$ heterotrimer, was activated with $50 \mu \mathrm{M}$ A-769662 (Fig. 5D). However, in the haploid cell lines, AMPK was activated upon $100 \mu \mathrm{M}$ A-769662 treatment independently of the expressed $\beta$-isoform (Fig. 5C). This result falls in line with other reports that showed that $\beta 2$-complexes can bind the A-769662 [58] with a dissociation constant higher than that of $\beta 1$ complexes but still in the low micromolar range [7] and that $\beta 2$-expressing tissues, like heart [59] and skeletal muscle $[10,60,61]$, were sensitive to the compound. Moreover, A-769662 can provide a certain degree of protection against dephosphorylation to $\beta 2$-containing heterotrimers in vitro [57]: this may be the mechanism at play in the $\beta 1$-deficient HAP1 cells as the phosphorylation degree of AMPK was increased upon treatment in this cell line 
compared to the WT and $\beta 2$-knockout cells (Fig. 5C). Therefore, even in the case of the well-established A-769662 compound, isoform preference remains a delicate puzzle. As described above, in the case of compound 6469172, a possible optimization strategy remains to be exploited based on our first results, which may lead to preference for $\beta 2$ containing complex, and therefore give a profile reciprocal to that of A-769662.

The identification of a cluster of six isoeugenol derivatives among the hit molecules provides with a head start for future studies on the structure-activity relationship. Isoeugenol is a natural compound found in essential oil of allspice (Pimenta dioica) or in Petunia flowers for instance [36]. In industry, this compound is widely found in fragrances and flavors, and is also used as precursor for the synthesis of vanillin [62, 63]. Although isoeugenol is classified as one of the most important fragrance allergens [62], the known applications of isoeugenol testify of a rather benign compound, which bodes well for the development of isoeugenol derivatives for possible use as drugs. Moreover, the risk profile may well be altered by the second ring found in all active derivatives of isoeugenol connected to the first by a carbon-oxygen linker of 6-7 atoms (Fig. 4, Table 1). As preliminary toxicological data, we did not find effects on cell viability (Supp. Fig. 4). To refine the definition of necessary features for both safety and potency, more derivatives of the identified compounds will need to be screened. In this future attempt, our protocol of in vitro screenings should come of use to quickly identify more active compounds.

Importantly, our screening is based exclusively on the CBM of AMPK and not on the heterotrimer, and thus relies on the assumption that AMPK-glycogen binding depends mainly on the CBM and little to no cooperative effect of the heterotrimer is needed. While we cannot formally exclude any influence on the CBM from the surrounding parts of the AMPK heterotrimer, several lines of evidence indicate the dominant contribution of CBM to glycogen binding. Firstly, for each of the $\beta$-subunit isoforms the reported dissociation constants for carbohydrates were similar for the isolated CBM [47] and the heterotrimer [64], i.e. only one to three-fold difference with better binding for the isolated CBM. Secondly, the CBM was recently reported to directly interact with the kinase domain of the $\alpha$-subunit and notably form the binding pocket needed to accommodate A-769662 $[7,46]$. However, $\beta$-cyclodextrin binding to the CBM prevented interaction with the kinase domain indicating that the carbohydrate-binding ability of AMPK does not rely on interaction with the $\alpha$-subunit [65]. Thirdly, hydrogen/ deuterium exchange mass spectroscopy showed that the $\beta$-cyclodextrin binding did not affect the $\alpha$ - and $\gamma$-subunits but only the CBM [58]. Finally, since our identified compound mimicked the effect of $\beta$-cyclodextrin binding on the immunoprecipitated AMPK heterotrimer, we remain confident that focusing on the CBM during the screening was a sensible approach to target the heterotrimer. 
The intended goal of the screening was to find a compound inducing the AMPK subcellular localization change from glycogen-bound to cytosolic free. Unexpectedly, our screening led to the identification of a new cellular activator of AMPK. The 6469172-stimulated AMPK activation was correlated with an increase in glucose and fatty acid uptake in a cardiomyocyte cell line. Similarly, also AICAR treatment and contraction cause an insulin-independent upregulation of glucose [66-68] and fatty acid [69] uptake in cardiac and skeletal muscle. Interestingly, these AMPK-dependent effects, as well as others including glycogen synthase phosphorylation, depend on glycogen levels [70, 71]. Despite some discrepancy, several papers reported an interdependence between AMPK activity and glycogen [3]. McBride et al. tried to reconcile the different views by suggesting that although AMPK can bind to both branching points of glycogen, only the $\alpha 1-6$ branches are inhibitory and thus different glycogen levels may lead to different effects on AMPK [17]. This hypothesis was thoroughly tested in cell-free experiments but could not be tested in cells. As mentioned above, another study reported disruption of the CBM and kinase domain interaction upon carbohydrate binding which may explain the inhibitory effect of glycogen on AMPK [65]. However, the precise mechanism through which glycogen branching points inhibits AMPK is largely unknown at this stage. In contrast to the branching points, 6469172 activates AMPK, but although we provide evidence that the compound does not act in an allosteric manner in cell-free kinase assays, the exact mechanism triggering this activation requires further clarification.

In summary, we established a new screening procedure for identification of compounds capable of preventing AMPK from binding to glycogen. The implementation of the screening uncovered an interesting cluster of isoeugenol derivatives able to compete the binding of the CBM domain of AMPK to the carbohydrate $\beta$-cyclodextrin and change the subcellular localization of the AMPK molecule. The compound 6469172 directly binds the recombinant CBM, activates cellular AMPK and triggers glucose and fatty acid uptake. Although many questions remain open, the duality of action on AMPK glycogen-binding and activity points to possible uses in models of metabolic disease. 


\section{References}

1. Yun, H. and Ha, J., AMP-activated protein kinase modulators: a patent review (2006 - 2010). Expert Opin Ther Pat, 2011. 21(7): p. 983-1005.

2. Rana, S., Blowers, E.C. and Natarajan, A., Small molecule adenosine 5'-monophosphate activated protein kinase (AMPK) modulators and human diseases. J Med Chem, 2015. 58(1): p. 2-29.

3. Viollet, B., Horman, S., Leclerc, J., Lantier, L., Foretz, M., Billaud, M., Giri, S. and Andreelli, F., AMPK inhibition in health and disease. Crit Rev Biochem Mol Biol, 2010. 45(4): p. 276-295.

4. Wang, S., Song, P. and Zou, M.H., AMP-activated protein kinase, stress responses and cardiovascular diseases. Clin Sci (Lond), 2012. 122(12): p. 555-573.

5. Hardie, D.G., AMPK--sensing energy while talking to other signaling pathways. Cell Metab, 2014. 20(6): p. 939-952.

6. Fogarty, S. and Hardie, D.G., Development of protein kinase activators: $A M P K$ as a target in metabolic disorders and cancer. Biochim Biophys Acta, 2010. 1804(3): p. 581-591.

7. Xiao, B., Sanders, M.J., Carmena, D., Bright, N.J., Haire, L.F., Underwood, E., Patel, B.R., Heath, R.B., Walker, P.A., Hallen, S., Giordanetto, F., Martin, S.R., Carling, D. and Gamblin, S.J., Structural basis of AMPK regulation by small molecule activators. Nat Commun, 2013. 4: p. 3017.

8. Sanders, M.J., Ali, Z.S., Hegarty, B.D., Heath, R., Snowden, M.A. and Carling, D., Defining the mechanism of activation of AMP-activated protein kinase by the small molecule A-769662, a member of the thienopyridone family. J Biol Chem, 2007. 282(45): p. 32539-32548.

9. Scott, J.W., Ling, N., Issa, S.M., Dite, T.A., O'Brien, M.T., Chen, Z.P., Galic, S., Langendorf, C.G., Steinberg, G.R., Kemp, B.E. and Oakhill, J.S., Small molecule drug A-769662 and AMP synergistically activate naive AMPK independent of upstream kinase signaling. Chem Biol, 2014. 21(5): p. 619-627.

10. Ducommun, S., Ford, R.J., Bultot, L., Deak, M., Bertrand, L., Kemp, B.E., Steinberg, G.R. and Sakamoto, K., Enhanced activation of cellular AMPK by dual-small molecule treatment: AICAR and A769662. Am J Physiol Endocrinol Metab, 2014. 306(6): p. E688-696.

11. Pang, T., Zhang, Z.S., Gu, M., Qiu, B.Y., Yu, L.F., Cao, P.R., Shao, W., Su, M.B., Li, J.Y., Nan, F.J. and Li, J., Small molecule antagonizes autoinhibition and activates AMP-activated protein kinase in cells. J Biol Chem, 2008. 283(23): p. 1605116060.

12. Jensen, T.E., Ross, F.A., Kleinert, M., Sylow, L., Knudsen, J.R., Gowans, G.J., Hardie, D.G. and Richter, E.A., PT-1 selectively activates AMPKgamma1 complexes in mouse skeletal muscle, but activates all three gamma subunit complexes in cultured human cells by inhibiting the respiratory chain. Biochem J, 2015. 467(3): p. 461472.
13. Hudson, E.R., Pan, D.A., James, J., Lucocq, J.M., Hawley, S.A., Green, K.A., Baba, O., Terashima, T. and Hardie, D.G., A novel domain in AMP-activated protein kinase causes glycogen storage bodies similar to those seen in hereditary cardiac arrhythmias. Current biology : CB, 2003. 13(10): p. 861-866.

14. Polekhina, G., Gupta, A., Michell, B.J., van Denderen, B., Murthy, S., Feil, S.C., Jennings, I.G., Campbell, D.J., Witters, L.A., Parker, M.W., Kemp, B.E. and Stapleton, D., AMPK beta subunit targets metabolic stress sensing to glycogen. Current biology : CB, 2003. 13(10): p. 867-871.

15. Oligschlaeger, Y., Miglianico, M., Chanda, D., Scholz, R., Thali, R.F., Tuerk, R., Stapleton, D.I., Gooley, P.R. and Neumann, D., The Recruitment of AMP-activated Protein Kinase to Glycogen Is Regulated by Autophosphorylation. J Biol Chem, 2015. 290(18): p. 11715-11728.

16. Jorgensen, S.B., Nielsen, J.N., Birk, J.B., Olsen, G.S., Viollet, B., Andreelli, F., Schjerling, P., Vaulont, S., Hardie, D.G., Hansen, B.F., Richter, E.A. and Wojtaszewski, J.F., The alpha2-5'AMP-activated protein kinase is a site 2 glycogen synthase kinase in skeletal muscle and is responsive to glucose loading. Diabetes, 2004. 53(12): p. 3074 3081.

17. McBride, A., Ghilagaber, S., Nikolaev, A. and Hardie, D.G., The glycogen-binding domain on the AMPK beta subunit allows the kinase to act as a glycogen sensor. Cell Metab, 2009. 9(1): p. 2334.

18. Polekhina, G., Gupta, A., van Denderen, B.J., Feil, S.C., Kemp, B.E., Stapleton, D. and Parker, M.W., Structural basis for glycogen recognition by AMP-activated protein kinase. Structure, 2005. 13(10): p. 1453-1462.

19. Joosten, R.P., Joosten, K., Cohen, S.X., Vriend, G. and Perrakis, A., Automatic rebuilding and optimization of crystallographic structures in the Protein Data Bank. Bioinformatics, 2011. 27(24): p. 3392-3398.

20. Krieger, E., Koraimann, G. and Vriend, G., Increasing the precision of comparative models with YASARA NOVA--a self-parameterizing force field. Proteins, 2002. 47(3): p. 393-402.

21. Laskowski, R.A., Moss, D.S. and Thornton, J.M., Main-chain bond lengths and bond angles in protein structures. J Mol Biol, 1993. 231(4): p. 1049-1067.

22. Vriend, G., WHAT IF: a molecular modeling and drug design program. Journal of molecular graphics, 1990. 8(1): p. 52-56, 29.

23. Pontius, J., Richelle, J. and Wodak, S.J., Deviations from standard atomic volumes as a quality measure for protein crystal structures. J Mol Biol, 1996. 264(1): p. 121-136.

24. Eisenberg, D., Luthy, R. and Bowie, J.U., VERIFY3D: assessment of protein models with threedimensional profiles. Methods in enzymology, 1997. 277: p. 396-404. 
25. Colovos, C. and Yeates, T.O., Verification of protein structures: patterns of nonbonded atomic interactions. Protein Sci, 1993. 2(9): p. 15111519.

26. Volkamer, A., Kuhn, D., Grombacher, T., Rippmann, F. and Rarey, M., Combining global and local measures for structure-based druggability predictions. J Chem Inf Model, 2012. 52(2): p. 360-372.

27. Larkin, M.A., Blackshields, G., Brown, N.P., Chenna, R., McGettigan, P.A., McWilliam, H., Valentin, F., Wallace, I.M., Wilm, A., Lopez, R., Thompson, J.D., Gibson, T.J. and Higgins, D.G., Clustal $W$ and Clustal X version 2.0. Bioinformatics, 2007. 23(21): p. 2947-2948.

28. Lagorce, D., Sperandio, O., Galons, H., Miteva, M.A. and Villoutreix, B.O., FAF-Drugs2: free ADME/tox filtering tool to assist drug discovery and chemical biology projects. BMC Bioinformatics, 2008. 9: p. 396.

29. Pollastri, M.P., Overview on the Rule of Five. Curr Protoc Pharmacol, 2010. Chapter 9: p. Unit 9 12.

30. Baell, J.B. and Holloway, G.A., New substructure filters for removal of pan assay interference compounds (PAINS) from screening libraries and for their exclusion in bioassays. J Med Chem, 2010. 53(7): p. 2719-2740.

31. Du, J., Bleylevens, I.W., Bitorina, A.V., Wichapong, K. and Nicolaes, G.A., Optimization of compound ranking for structure-based virtual ligand screening using an established FRED-Surflex consensus approach. Chem Biol Drug Des, 2014. 83(1): p. 37-51.

32. Zarzycka, B., Seijkens, T., Nabuurs, S.B., Ritschel, T., Grommes, J., Soehnlein, O., Schrijver, R., van Tiel, C.M., Hackeng, T.M., Weber, C., Giehler, F., Kieser, A., Lutgens, E., Vriend, G. and Nicolaes, G.A., Discovery of small molecule CD40-TRAF6 inhibitors. J Chem Inf Model, 2015. 55(2): p. 294-307.

33. Miteva, M.A., Lee, W.H., Montes, M.O. and Villoutreix, B.O., Fast structure-based virtual ligand screening combining FRED, DOCK, and Surflex. Journal of medicinal chemistry, 2005. 48(19): p. 6012-6022.

34. Berthold, M., Cebron, N., Dill, F., Gabriel, T., Kötter, T., Meinl, T., Ohl, P., Sieb, C., Thiel, K. and Wiswedel, B., KNIME: The Konstanz Information Miner, in Data Analysis, Machine Learning and Applications, C. Preisach, et al., Editors. 2008, Springer Berlin Heidelberg. p. 319-326.

35. Beisken, S., Meinl, T., Wiswedel, B., de Figueiredo, L.F., Berthold, M. and Steinbeck, C., KNIMECDK: Workflow-driven cheminformatics. BMC Bioinformatics, 2013. 14: p. 257.

36. Backman, T.W., Cao, Y. and Girke, T., ChemMine tools: an online service for analyzing and clustering small molecules. Nucleic acids research, 2011. 39(Web Server issue): p. W486-491.

37. Seeliger, D. and de Groot, B.L., Ligand docking and binding site analysis with PyMOL and Autodock/Vina. J Comput Aided Mol Des, 2010. 24(5): p. 417-422.
38. Korth, M., Third-Generation Hydrogen-Bonding Corrections for Semiempirical QM Methods and Force Fields. J Chem Theory Comput, 2010. 6(12): p. 3808-3816.

39. Karaman, B. and Sippl, W., Docking and binding free energy calculations of sirtuin inhibitors. Eur J Med Chem, 2015. 93: p. 584-598.

40. Miller, B.R., McGee, T.D., Swails, J.M., Homeyer, N., Gohlke, H. and Roitberg, A.E., MMPBSA.py: An Efficient Program for End-State Free Energy Calculations. J Chem Theory Comput, 2012. 8(9): p. 3314-3321.

41. Case DA, Darden TA, Cheatham TE, I., Simmerling CL, Wang J, Duke RE, Luo R, Walker RC, Zhang W, Merz KM, Roberts B, Hayik S, Roitberg A, Seabra G, Swails J, Götz AW, Kolossváry I, Wong KF, Pae-sani F, Vanicek J, Wolf RM, Liu J, Wu X, Brozell SR, Steinbrecher T, Gohlke H, Cai Q Ye X, Wang J, Hsieh MJ, Cui G, Roe DR, Mathews DH, Seetin MG, Salomon-Ferrer R, Sagui C, Babin V, Luchko T, Gusarov S, Ko-valenko A and PA, K., Amber 12. 2012: University of California, San Francisco; San Francisco, CA.

42. Wichapong, K., Lawson, M., Pianwanit, S., Kokpol, S. and Sippl, W., Postprocessing of proteinligand docking poses using linear response MMPB/SA: application to Wee1 kinase inhibitors. J Chem Inf Model, 2010. 50(9): p. 1574-1588.

43. Wichapong, K., Rohe, A., Platzer, C., Slynko, I., Erdmann, F., Schmidt, M. and Sippl, W., Application of docking and QM/MM-GBSA rescoring to screen for novel Myt1 kinase inhibitors. J Chem Inf Model, 2014. 54(3): p. 881-893.

44. Dirkx, E., Schwenk, R.W., Coumans, W.A., Hoebers, N., Angin, Y., Viollet, B., Bonen, A., van Eys, G.J., Glatz, J.F. and Luiken, J.J., Protein kinase D1 is essential for contraction-induced glucose uptake but is not involved in fatty acid uptake into cardiomyocytes. J Biol Chem, 2012. 287(8): p. 5871-5881.

45. Claycomb, W.C., Lanson, N.A., Jr., Stallworth, B.S., Egeland, D.B., Delcarpio, J.B., Bahinski, A. and Izzo, N.J., Jr., HL-1 cells: a cardiac muscle cell line that contracts and retains phenotypic characteristics of the adult cardiomyocyte. Proceedings of the National Academy of Sciences of the United States of America, 1998. 95(6): p. 2979-2984.

46. Calabrese, M.F., Rajamohan, F., Harris, M.S., Caspers, N.L., Magyar, R., Withka, J.M., Wang, H., Borzilleri, K.A., Sahasrabudhe, P.V., Hoth, L.R., Geoghegan, K.F., Han, S., Brown, J., Subashi, T.A., Reyes, A.R., Frisbie, R.K., Ward, J., Miller, R.A., Landro, J.A., Londregan, A.T., Carpino, P.A., Cabral, S., Smith, A.C., Conn, E.L., Cameron, K.O., Qiu, X. and Kurumbail, R.G., Structural basis for AMPK activation: natural and synthetic ligands regulate kinase activity from opposite poles by different molecular mechanisms. Structure, 2014. 22(8): p. 1161-1172.

47. Koay, A., Woodcroft, B., Petrie, E.J., Yue, H., Emanuelle, S., Bieri, M., Bailey, M.F., Hargreaves, M., Park, J.T., Park, K.H., Ralph, S., Neumann, D., Stapleton, D. and Gooley, P.R., AMPK beta subunits display isoform specific affinities for carbo- 
hydrates. FEBS Lett, 2010. 584(15): p. 34993503.

48. Aretz, J., Wamhoff, E.C., Hanske, J., Heymann, D. and Rademacher, C., Computational and experimental prediction of human $C$-type lectin receptor druggability. Front Immunol, 2014. 5: p. 323.

49. Uciechowska, U., Schemies, J., Scharfe, M., Lawson, M., Wichapong, K., Jung, M. and Sippl, W., Binding free energy calculations and biological testing of novel thiobarbiturates as inhibitors of the human NAD+ dependent histone deacetylase Sirt2. MedChemComm, 2012. 3(2): p. 167-173.

50. Wang, J., Morin, P., Wang, W. and Kollman, P.A., Use of MM-PBSA in reproducing the binding free energies to HIV-1 RT of TIBO derivatives and predicting the binding mode to HIV-1 RT of efavirenz by docking and MM-PBSA. J Am Chem Soc, 2001. 123(22): p. 5221-5230.

51. Gleeson, M.P. and Gleeson, D., QM/MM as a tool in fragment based drug discovery. A cross-docking, rescoring study of kinase inhibitors. J Chem Inf Model, 2009. 49(6): p. 1437-1448.

52. Segers, K., Sperandio, O., Sack, M., Fischer, R., Miteva, M.A., Rosing, J., Nicolaes, G.A. and Villoutreix, B.O., Design of protein membrane interaction inhibitors by virtual ligand screening, proof of concept with the C2 domain of factor $V$. Proceedings of the National Academy of Sciences of the United States of America, 2007. 104(31): p. 12697-12702.

53. Prats, C., Cadefau, J.A., Cusso, R., Qvortrup, K., Nielsen, J.N., Wojtaszewski, J.F., Hardie, D.G., Stewart, G., Hansen, B.F. and Ploug, T., Phosphorylation-dependent translocation of glycogen synthase to a novel structure during glycogen resynthesis. J Biol Chem, 2005. 280(24): p. 23165-23172.

54. Phatak, S.S., Stephan, C.C. and Cavasotto, C.N., High-throughput and in silico screenings in drug discovery. Expert opinion on drug discovery, 2009. 4(9): p. 947-959.

55. Scott, J.W., van Denderen, B.J., Jorgensen, S.B., Honeyman, J.E., Steinberg, G.R., Oakhill, J.S., Iseli, T.J., Koay, A., Gooley, P.R., Stapleton, D. and Kemp, B.E., Thienopyridone drugs are selective activators of AMP-activated protein kinase beta1-containing complexes. Chem Biol, 2008. 15(11): p. 1220-1230.

56. Treebak, J.T., Birk, J.B., Hansen, B.F., Olsen, G.S. and Wojtaszewski, J.F., A-769662 activates AMPK beta1-containing complexes but induces glucose uptake through a PI3-kinase-dependent pathway in mouse skeletal muscle. Am J Physiol Cell Physiol, 2009. 297(4): p. C1041-1052.

57. Hawley, S.A., Fullerton, M.D., Ross, F.A., Schertzer, J.D., Chevtzoff, C., Walker, K.J., Peggie, M.W., Zibrova, D., Green, K.A., Mustard, K.J., Kemp, B.E., Sakamoto, K., Steinberg, G.R. and Hardie, D.G., The ancient drug salicylate directly activates AMP-activated protein kinase. Science, 2012. 336(6083): p. 918-922.
58. Landgraf, R.R., Goswami, D., Rajamohan, F., Harris, M.S., Calabrese, M.F., Hoth, L.R., Magyar, R., Pascal, B.D., Chalmers, M.J., Busby, S.A., Kurumbail, R.G. and Griffin, P.R., Activation of AMP-activated protein kinase revealed by hydrogen/ deuterium exchange mass spectrometry. Structure, 2013. 21(11): p. 1942-1953.

59. Kim, A.S., Miller, E.J., Wright, T.M., Li, J., Qi, D., Atsina, K., Zaha, V., Sakamoto, K. and Young, L.H., A small molecule AMPK activator protects the heart against ischemia-reperfusion injury. J Mol Cell Cardiol, 2011. 51(1): p. 24-32.

60. Guigas, B., Sakamoto, K., Taleux, N., Reyna, S.M., Musi, N., Viollet, B. and Hue, L., Beyond AICA riboside: in search of new specific AMP-activated protein kinase activators. IUBMB Life, 2009. 61(1): p. 18-26.

61. Goransson, O., McBride, A., Hawley, S.A., Ross, F.A., Shpiro, N., Foretz, M., Viollet, B., Hardie, D.G. and Sakamoto, K., Mechanism of action of A-769662, a valuable tool for activation of AMPactivated protein kinase. J Biol Chem, 2007. 282(45): p. 32549-32560.

62. Dela, F., Prats, C. and Helge, J.W., Exercise interventions to prevent and manage type 2 diabetes: physiological mechanisms. Med Sport Sci, 2014. 60: p. 36-47.

63. Kerzmann, A., Fuhrmann, J., Kohlbacher, O. and Neumann, D., BALLDock/SLICK: a new method for protein-carbohydrate docking. J Chem Inf Model, 2008. 48(8): p. 1616-1625.

64. Bieri, M., Mobbs, J.I., Koay, A., Louey, G., Mok, Y.F. Hatters, D.M., Park, J.T., Park, K.H., Neumann, D., Stapleton, D. and Gooley, P.R., AMP-activated protein kinase beta-subunit requires internal motion for optimal carbohydrate binding. Biophys J, 2012. 102(2): p. 305-314.

65. Li, X., Wang, L., Zhou, X.E., Ke, J., de Waal, P.W., $\mathrm{Gu}, \mathrm{X} .$, Tan, M.H., Wang, D., Wu, D., Xu, H.E. and Melcher, K., Structural basis of AMPK regulation by adenine nucleotides and glycogen. Cell research, 2015. 25(1): p. 50-66.

66. Hunter, R.W., Treebak, J.T., Wojtaszewski, J.F. and Sakamoto, K., Molecular mechanism by which AMP-activated protein kinase activation promotes glycogen accumulation in muscle. Diabetes, 2011.60(3): p. 766-774.

67. Miyamoto, L., Toyoda, T., Hayashi, T., Yonemitsu, S., Nakano, M., Tanaka, S., Ebihara, K., Masuzaki, H., Hosoda, K., Ogawa, Y., Inoue, G., Fushiki, T. and Nakao, K., Effect of acute activation of 5'-AMP-activated protein kinase on glycogen regulation in isolated rat skeletal muscle. J Appl Physiol (1985), 2007. 102(3): p. 1007-1013.

68. Russell, R.R., 3rd, Bergeron, R., Shulman, G.I. and Young, L.H., Translocation of myocardial GLUT-4 and increased glucose uptake through activation of AMPK by AICAR. Am J Physiol, 1999. 277(2 Pt 2): p. H643-649.

69. Luiken, J.J., Coort, S.L., Willems, J., Coumans, W.A., Bonen, A., van der Vusse, G.J. and Glatz, J.F., Contraction-induced fatty acid translocase/ CD36 translocation in rat cardiac myocytes is 
mediated through AMP-activated protein kinase signaling. Diabetes, 2003. 52(7): p. 1627-1634.

70. Wojtaszewski, J.F., Jorgensen, S.B., Hellsten, Y., Hardie, D.G. and Richter, E.A., Glycogen-dependent effects of 5-aminoimidazole-4-carboxamide (AICA)-riboside on AMP-activated protein kinase and glycogen synthase activities in rat skeletal muscle. Diabetes, 2002. 51(2): p. 284-292.
71. Nielsen, J.N., Derave, W., Kristiansen, S., Ralston, E., Ploug, T. and Richter, E.A., Glycogen synthase localization and activity in rat skeletal muscle is strongly dependent on glycogen content. J Physiol, 2001. 531(Pt 3): p. 757-769. 


\section{Supplementary figures}

A

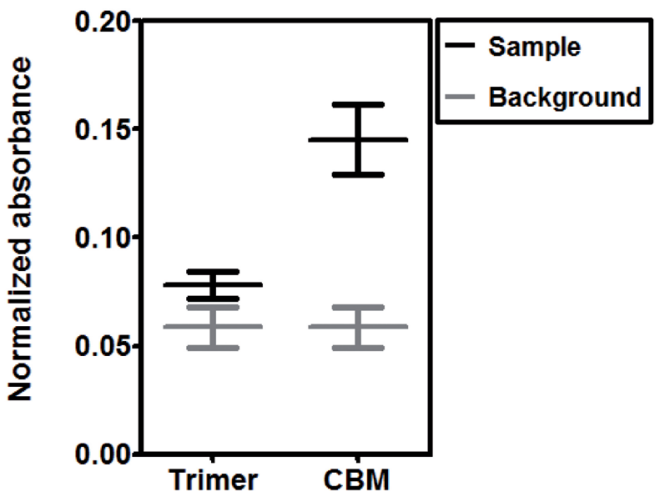

B
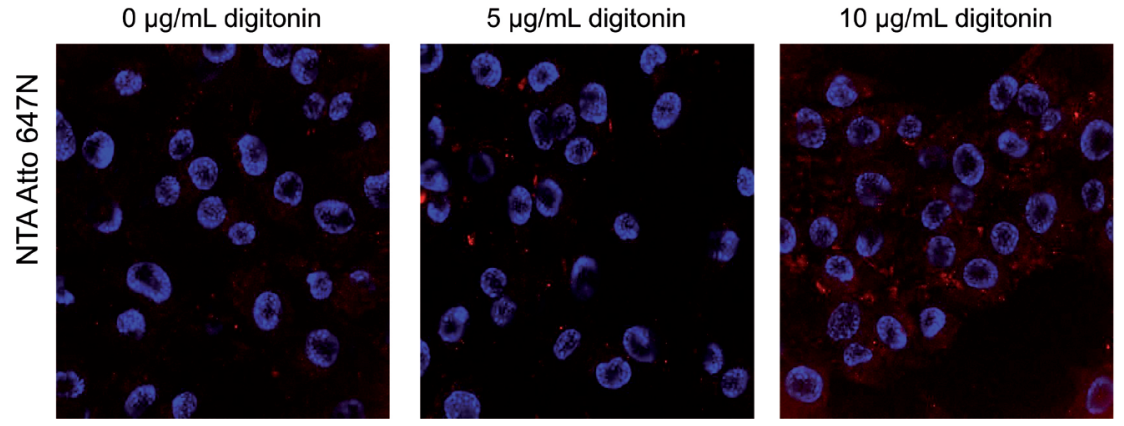

$15 \mu \mathrm{g} / \mathrm{mL}$ digitonin

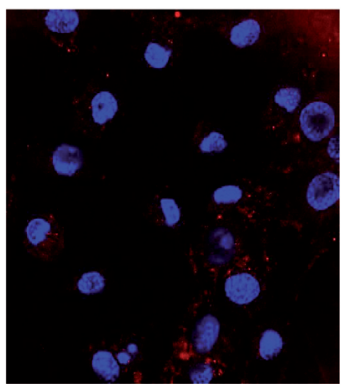

$20 \mu \mathrm{g} / \mathrm{mL}$ digitonin

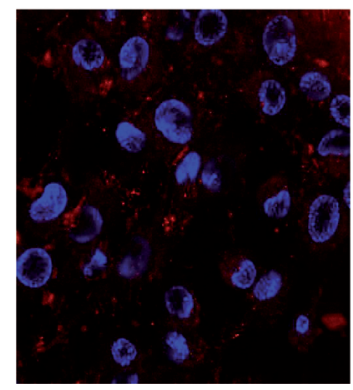

$25 \mu \mathrm{g} / \mathrm{mL}$ digitonin

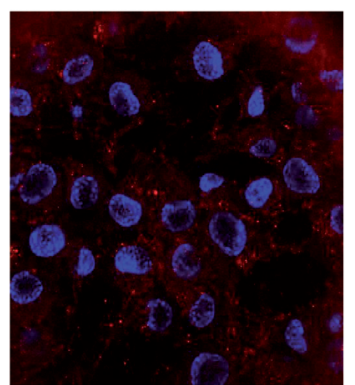

\section{Supplementary Figure 1.}

(A) Normalized absorbance (sample in black and background in grey) of $100 \mu \mathrm{M}$ maltoheptaose in the carbohydrate-binding competition assay, using either the $\alpha 1 \beta 2 \gamma 1$ AMPK heterotrimer or the isolated СВM $\beta 2 ; n=3$. (B) Dose-dependent effect of digitonin on permeabilization. $\mathrm{C} 2 \mathrm{C} 12$ were treated with a constant concentration of the non-permeable substance NTA-Atto 647N (Sigma-Aldrich) and with increasing doses of digitonin (0 to 25 $\mu \mathrm{g} / \mathrm{mL}$ ). After treatment, cells were washed once in PBS and imaged with a fluorescent microscope. 


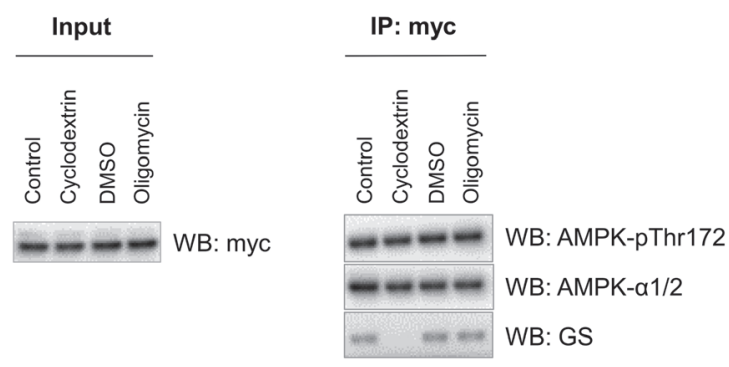

\section{Supplementary Figure 2.}

Lysate of AMPK-overexpressing mammalian cells untreated or treated with either vehicle $(0.5 \%$ DMSO), $5 \mu \mathrm{M}$ of oligomycin, or $5 \mathrm{mM} \beta$-cyclodextrin (left panel). Immunoprecipitation of AMPK heterotrimer was performed with the myc antibody following the treatment (right panel). The used antibodies recognize total level of myc tagged-protein (myc), total level of glycogen synthase (GS), total level of AMPK- $\alpha$ subunit (AMPK- $\alpha 1 / 2$ ), and phosphorylation status of AMPK at Thr172 (AMPK-pThr172); $n=2$
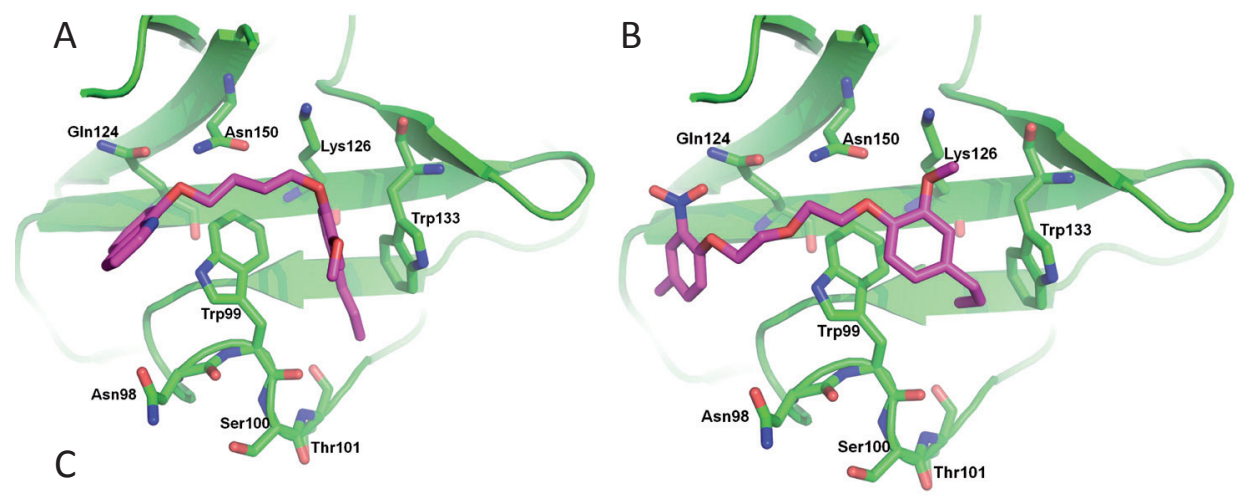

\begin{tabular}{|c|c|c|c|c|c|c|c|}
\hline \multirow{2}{*}{ Compound ID } & \multirow{2}{*}{$\begin{array}{c}\mathrm{K}_{\mathrm{D}} \\
(\mu \mathrm{M})\end{array}$} & \multirow{2}{*}{$\begin{array}{c}\Delta G_{\text {exp }} \\
(\mathrm{kcal} / \mathrm{mol})\end{array}$} & \multirow{2}{*}{$\begin{array}{l}\text { Binding } \\
\text { Mode }\end{array}$} & \multicolumn{4}{|c|}{$\begin{array}{l}\text { Binding Free Energy (kcal/mol) } \\
\text { derived from different methods }\end{array}$} \\
\hline & & & & MM/GBSA & MM/PBSA & $\begin{array}{l}\text { QM/MM-GBSA } \\
\text { (AM1-DH+) }\end{array}$ & $\begin{array}{l}\text { QM/MM-GBSA } \\
\text { (PM6-DH+) }\end{array}$ \\
\hline \multirow{2}{*}{6456019} & \multirow{2}{*}{5.44} & \multirow{2}{*}{-7.23} & $\begin{array}{c}1 \\
(\text { Fig } 4 \mathrm{~A})\end{array}$ & -21.47 & -19.51 & -37.36 & -7.93 \\
\hline & & & $\begin{array}{c}2 \\
\text { (Supp Fig3A) }\end{array}$ & 6.21 & 19.82 & -5.01 & 20.24 \\
\hline \multirow{2}{*}{6469172} & \multirow{2}{*}{25.40} & \multirow{2}{*}{-6.31} & $\begin{array}{c}1 \\
\text { (Fig 4B) }\end{array}$ & -12.39 & -11.52 & -29.60 & -6.57 \\
\hline & & & $\begin{array}{c}2 \\
\text { (Supp Fig3B) }\end{array}$ & -8.16 & -5.34 & -24.91 & -0.92 \\
\hline
\end{tabular}

\section{Supplementary Figure 3.}

(A-B) Alternative predicted binding mode of 6469172 (A) and 6456019 (B) in CBM 32 (defined as mode 2). (C) Experimental and predicted binding free energy calculations for both binding modes of 6469172 and 6456019 with different methods. 


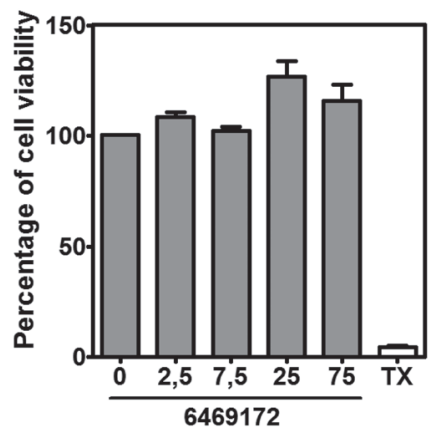

\section{Supplementary Figure 4.}

Toxicity measurement by MTT method with increasing amount of 6469172 (doses in $\mu \mathrm{M}$ with constant level of vehicle, i.e. 1\% DMSO). Absorbances were normalized to untreated cell absorbance, which gave $100 \%$ cell viability. Positive control was 1\% triton-X (TX). 


Chapter 6

General discussion 


\section{Thesis main findings}

Metabolic diseases such as insulin resistance and in a more advanced level, diabetes, are complex disorders with causes and consequences that are difficult to disentangle [1]. Glucose metabolism is a major contributor to the state of dysregulation as can be judged by the associated high blood glucose and decreased glycogen synthesis in key organs. Among the many proteins involved in glycogen maintenance and regulation, AMP-activated protein kinase (AMPK) is a well-known pivotal regulator of metabolism and a recognized target for metabolic disease treatment [2]. AMPK binds to glycogen through its carbohydrate-binding module (CBM) located on the $\beta$-subunit of the enzyme. Although this domain was discovered and even crystallized more than a decade ago [3, 4], the knowledge on its influence on AMPK regulation remains limited.

In this thesis, we investigated the regulation and role of the AMPK-CBM in the context of glucose metabolism. Furthermore we developed a protocol for screening compounds targeting the AMPK-CBM. Accordingly, the main findings of this thesis are:

1. A safe and efficient pharmacological targeting of AMPK still eludes the field but considering the involvement of AMPK in a great number of diseases, the challenge of finding novel approaches to directly modulate AMPK is worth taking up. (Chapter 2)

2. Computer-aided drug design could provide the necessary tools to undertake such targeting project, and particularly that of isoform specific targeting, as the available data are currently under-exploited. (Chapter 2)

3. AMPK-glycogen binding is prevented by autophosphorylation within AMPKCBM on $\beta$-Thr148. (Chapter 3)

4. This regulatory autophosphorylation mechanism impacts AMPK subcellular localization and may be of importance to regulate glycogen metabolism. (Chapter 3)

5. The interaction between the protein phosphatase 1 targeting subunit R6 and AMPK is up-regulated under low glucose conditions, which correlates with a low glycogen content. (Chapter 4)

6. The binding of these partners is further enhanced by treatment with an AMPK activator which induces Thr148 autophosphorylation. (Chapter 4)

7. Our protocol of virtual screening followed by a two-step in vitro screen led to the identification of small molecules targeting the CBM of AMPK. (Chapter 5)

8. The main hit molecule, 6469172, binds the CBM of AMPK directly and activates the kinase in a $\beta$-isoform independent manner. (Chapter 5) 
With this thesis, exciting new elements of AMPK regulation have been unveiled and a new compound for AMPK modulation has been added to the toolbox through the advocated strategy of rationalized structure-based drug design. In the present chapter these new results are placed within the context of other findings to give perspectives as to how the presented work could be followed up. Specifically, the results of the different experimental chapters are brought together and seen from a broader point of view, especially in the context of glycogen metabolism and metabolic disease. Next, the general involvement of carbohydrate-binding modules in cellular processes and how their properties could be exploited is discussed. Finally, implications that follow the discovery of the AMPK-CBM displacing compounds are presented in terms of their possible applications and further development.

\section{Significance of the new findings in metabolic health and disease}

In the introduction of this thesis, the relevance of AMPK as therapeutic target for metabolic diseases was highlighted through the review of the known beneficial effects of AMPK activation. Even though indirect activators like metformin are routinely used for the treatment of diabetic patients, these molecules still have drawbacks notably that of being basically mitochondrial poisons, which underscores the need to identify alternative approaches in diabetes treatment. Furthermore, the known direct AMPK activators still leave room for improvement and possible innovation since they deeply miss needed drug-like properties and also a desired specificity (Chapter 2). For a successful drug design process, target validation is of major importance and therefore the more knowledge is gathered the likelier the success [5]. In this thesis we unveil new mechanisms and regulation in the interplay between AMPK and glycogen, notably with regards to control of glycogen resynthesis. Importantly, the novel data presented here may have consequences for the application of the newly identified AMPK-CBM targeting compound.

\section{AMPK-glycogen interplay: the case of glycogen resynthesis}

Glycogen forms an energy store which is submitted to alternate phases of degradation and (re)synthesis, both during fasting and refeeding, and during and after exercise. The two main storage organs are liver and skeletal muscle [6]. In muscle, glycogen is not a uniform pool: different subcellular stocks exist (intramyofibrillar, intermyofibrillar and subsarcolemmal) with each separate roles and regulation [7]. For instance, the intramyofibrillar pool is the first to be mobilized upon prolonged exercise and is replenished quickly afterwards $[7,8]$. Whereas the overall rate of synthesis is primarily regulated by glucose import, how the separate regulation of the different subcellular pools is carried out is not entirely known [9]. Subcellular translocation of key enzymes 
emerges as an important factor to coordinate this differentiated muscle glycogen storage.

Among the involved enzymes, glycogen synthase has a fairly complex regulation with multiple sites of phosphorylation, allosteric activation by glucose-6-phosphate and translocation abilities, which makes the assessment of its in situ activity difficult [9]. Actually, glycogen synthase phosphorylation influences both activity (mostly by inhibition) and subcellular localization [9]. For instance phosphorylation of site $1 \mathrm{~b}$ results in translocation of glycogen synthase to intramyofibrillar particles while phosphorylations of sites 2 and 2 a associate with subsarcolemmal and intermyofibrillar localization [8]. During an exercise-mimicking stimulation of rabbit muscle, although glycogen synthase activity was increased very rapidly, the resynthesis of glycogen stores began only after several hours of exercise [10]. The subcellular localization of glycogen synthase correlated more closely with this time frame, indicating that at least in this case, regulation of localization prevails over direct change in activity.

Interestingly, AMPK was reported to be one of the kinases which can phosphorylate glycogen synthase on site 2, prompting the sequential phosphorylation of site $2 \mathrm{a}$ by casein kinase $[8,9,11]$. AMPK action therefore both inhibits and presumably targets glycogen synthase towards intermyofibrillar and subsarcolemmal stores. However, as mentioned above, knowing the phosphorylation status of glycogen synthase alone is not sufficient to interpret activity [9]. For instance, pharmacological AMPK activation results in increased glycogen synthesis under high glucose conditions, because the rise in glucose-6-phosphate which follows the AMPK-induced glucose uptake allosterically activates glycogen synthase, independently of the enzyme phosphorylation status [12] In Chapter 3, activation of AMPK by different activators (which therefore reduces the risk of looking at AMPK unspecific effects) consistently decreases glycogen levels under "normal" glucose conditions (i.e. $5.5 \mathrm{mM}$ ). The mutation of AMPK- $\beta$ Thr148 to a phosphorylation mimicking mutant aspartate (T148D mutation) prevents the decrease in glycogen synthesis, indicating that AMPK subcellular localization at glycogen was mandatory for the AMPK-induced suppression of glycogen synthesis. Therefore, on top of the above-described importance of glycogen synthase localization, the attachment of AMPK at glycogen also plays a role in regulating glycogen synthesis.

Additionally, this regulation by autophosphorylation of Thr148 indicates a potential role as safety net, banning AMPK from glycogen when it is not needed there. Firstly under conditions that consume glycogen (fasting or exercise), AMPK needs to be activated, but the attachment of AMPK to the partially degraded particles would theoreticallyinhibitAMPK[13].Thereforetheregulation byThr148autophosphorylation prevents the glycogen-induced inhibition of AMPK to take place under these conditions. This could be of primordial importance since AMPK shows a better affinity for 
oligosaccharide that have the same feature as degraded granules (i.e. with $\alpha-1,6$ branching), which suggests that degraded glycogen could attract more AMPK than basal conditions [14]. Secondly, the more glycogen is depleted, the more Thr148 will be autophosphorylated as AMPK comes loose from the particles (Chapter 4). In this view, the AMPK Thr148 autophosphorylation accumulated during stress-associated glycogen depletion would prevent for a too quick reattachment of AMPK to the glycogen granules in which glycogen synthesis needs to be maintained after the stress has ceased.

The dynamic interaction of AMPK with the protein phosphatase 1 (PP1) targeting subunit, R6 (Chapter 4), takes also part in this picture of safety net allowing for a smooth transition from glycogen depleting conditions to resynthesis of glycogen levels. As AMPK comes loose from glycogen due to the ongoing particle degradation and increased Thr148 autophosphorylation, AMPK and R6 interact. Presumably, this interaction is important to bring the phosphatase PP1 in close proximity of AMPK to dephosphorylate the kinase once the stress has ceased and the replacement of AMP by ATP in the regulatory subunit of AMPK relieves the Thr172 phosphorylation protection.

Altogether our data from Chapters 3 and 4 present a safety net mechanism which is put in place upon stress by autophosphorylation of Thr148 and subsequent association with R6 to prepare for the future cessation of the stress when a quick adaptation will be required. Our understanding of AMPK and glycogen interplay thus deepened with the novel data presented in this thesis. A limitation of these results is however the representation of glycogen as a uniform pool. Further refinement of synthesis regulation at the subcellular level may prove interesting. Specifically, among the different pools of muscle glycogen, the preferential location of AMPK and the signals that regulate its translocation are not known and may play a role in glycogen synthesis regulation.

\section{Implication in glycogen modulation: an opportunity for drug design}

As described in the section above, our new findings further delineate the role of AMPK in glycogen resynthesis, which could influence further drug design opportunities.

Exercise is a recognized successful intervention to prevent progression from impaired glucose sensitivity to full-blown type 2 diabetes [15]. Among the symptoms that are helped by such change in lifestyle, a meta-analysis concluded that physical training significantly lowered blood glucose of type 2 diabetes patients [15]. Not surprisingly, skeletal muscle is one of the major contributors to blood glucose clearance: increased glucose uptake and glycogenolysis firstly fuel the glycolysis pathway during the exercise bout. Then, after exercise more circulating glucose is used as glycogen stores need to be replenished. Interestingly, after a glycogen-depleting exercise, the level of resynthesized glycogen can be $50-80 \%$ higher than the initial stock [7]. 
Therefore, muscle has potential to store an increased amount of glycogen: this overaccumulation could constitute a form of storage to reduce the excess circulating glucose which is associated with insulin resistance.

Since compound 6469172, identified in Chapter 5, mimics the effect of Thr148 phosphorylation by preventing the attachment of AMPK to glycogen (which should maintain glycogen synthesis) and yet promotes activation of the kinase (which, with classical activators, is known to stop glycogen synthesis), the effect on glycogen (re) synthesis should be interesting. Similarly, compound 6469172 could also intervene on AMPK-R6 interaction since the mutant T148D which has the same theoretical effect as the compound on AMPK glycogen attachment disrupted the interaction. However, the conditions under which glycogen is depleted are associated with increased Thr148 phosphorylation. Since this site is within the carbohydrate-binding pocket, we cannot know whether our compound could still bind to the pocket in that state. Therefore, further investigations need to be carried out to determine whether use of the compound could be a good approach to boost glycogen (re)synthesis, in a manner similar to the T148D mutant (Chapter 3).

Alternatively, since compound 6469172 can mimic the effect of Thr148 autophosphorylation on AMPK glycogen attachment, the study of the occurrence of this modification under pathological conditions could define potential applications for the compound. At this stage, we can only speculate on what these conditions could be, but apart from metabolic diseases, investigating the role of this modification in glycogen-related disorders such as Wolff-Parkinson-White or Lafora Disease could be a good start. These hereditary diseases are respectively a cardiomyopathy correlated with increased glycogen storage and caused by AMPK mutation [16], and a neurodegenerative disease where polysaccharide accumulation in the brain is caused by loss of function of either malin or laforin with a potential link with AMPK regulation [17].

In summary, the delineated role of Thr148 phosphorylation in glycogen storage could define a new strategy for metabolic disease treatment, possibly with the aid of the reported new compound class. Alternatively, investigation of pathologies in which Thr148 phosphorylation is low could determine applications for our newly identified compound.

\section{Carbohydrate-binding modules}

Apart from the study of AMPK and its role in metabolic regulation, a central aspect of this thesis was the study of carbohydrate-binding modules, as are found both in AMPK and R6. Despite their absence of catalytic activity, these domains are far from being accessory and their biological roles are already being exploited in industry. The 
study of CBM in this thesis led to two discoveries that were not part of the previous chapters but are discussed below: (i) a potential new application for CBM in laboratories and (ii) an as yet unexplained physiological role in glycogen metabolism.

\section{An exploitable use for AMPK-CBM: live cell glycogen sensor}

Carbohydrate-binding modules (CBM) are defined as contiguous amino acid sequences with a discrete fold and capacity to bind carbohydrate [18]. Behind this fairly simple definition, the identified CBM form a class of domains with a wide diversity of carbohydrate ligands, from complex polysaccharides such as glycogen, starch and cellulose, to small sugars like fructose and lipopolysaccharide [19]. Generally found in enzymes that have direct hydrolytic activity towards carbohydrates, CBM present in single or multiple occurrences are known to enhance catalytic activity notably by bringing the enzyme in close proximity with its substrate and by targeting very specific substrates thanks to their high ligand specificity [20]. Hence, despite being devoid of catalytic activity themselves, their physiological role in directing carbohydrate-active enzymes is of primordial importance. These CBM characteristics attracted interest from industries where they are routinely used as fusion partners in recombinant protein expression to increase activity and stability of catalytic enzymes notably in paper, food and textile industries $[18,20]$. In research laboratories, CBM fusion proteins are further used, for instance, for affinity-based recombinant protein purifications and preparation of micro-arrays [20]. Our work with CBM in this thesis led to the idea of a new application for glycogen staining. To this date, the available methods to assess cellular glycogen content require lysis or fixation of the sample, which is of course suboptimal since the measurement of glycogen with these methods cannot be combined with any other assay or cannot be repeated over time. We therefore intended to develop a novel glycogen sensor which would be applicable to live cells.

The data obtained with GFP-tagged AMPK-CBM argued that this domain could be a good base for this new glycogen sensor. Indeed, on top of a high co-localization with glycogen (Chapter 3), the use of the imaging method developed in Chapter 5 (with automated microscope pictures and automated recognition of GFP speckles within each cell) makes the quantification of GFP speckles an indirect measurement of glycogen levels: the use of the glycogen-depleting agent forskolin significantly diminished the number of GFP speckles (Chapter 5) in a way similar to the reduction seen with the biochemical measurement of glycogen (Chapter 3). Using a glycogen antibody, we further co-stained the GFP-CBM overexpressing cells after treatment with forskolin or vehicle and quantified the number of speckles for both GFP and glycogen staining: a high correlation was found between the two staining methods, indicating that the GFP tagged CBM quantification was as valuable to assess glycogen levels as the 
A

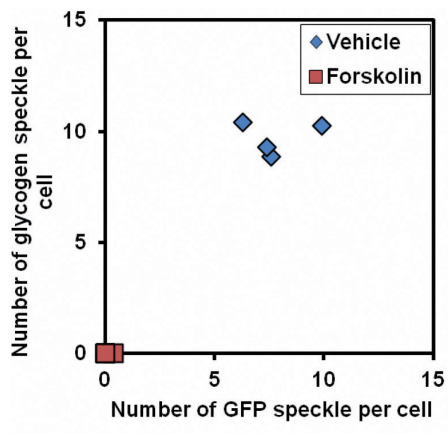

B

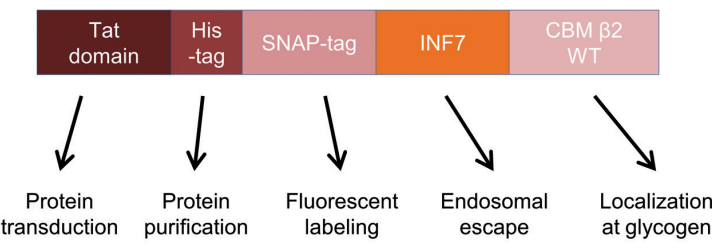

C

Incubation 3h

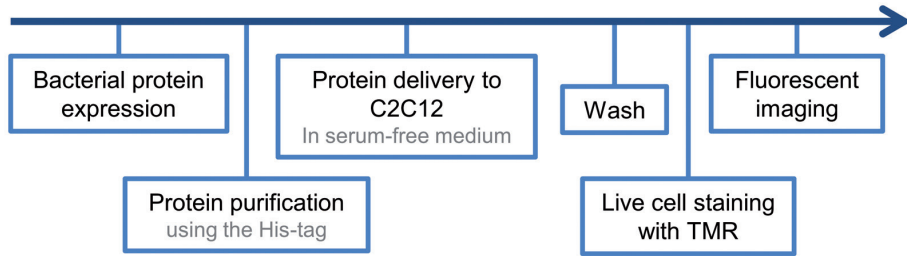

$\mathrm{D}$

C2C12 cell treated with TAT-tagged CBM-WT

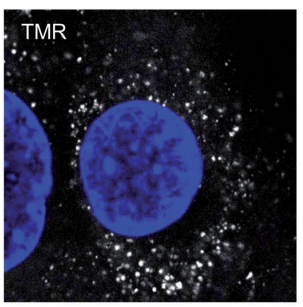

Untreated $\mathrm{C} 2 \mathrm{C} 12$ cell

C2C12 cell infected with GFP-tagged CBM-WT
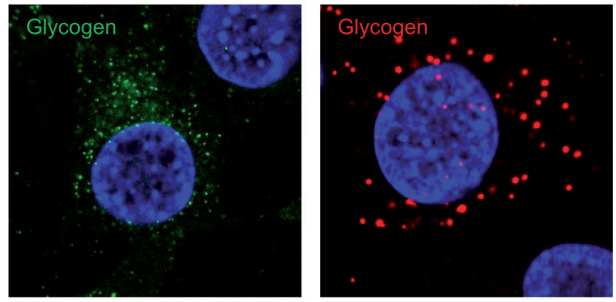

Figure 1. Development of a live cell glycogen sensor.

(A) Quantification of speckle number for both GFP staining and glycogen staining (fluorescent immunostaining with glycogen antibody) on GFP-tagged AMPK-CBM wild type (WT) infected C2C12 cells. Cells were treated for eight hours with either $100 \mu \mathrm{M}$ forskolin (blue diamonds) or equivalent vehicle (1\% DMSO, red squares). $\mathrm{N}=4$, each repeat represents the average of $>150$ measured cells. (B) Outline of the designed construct for live cell glycogen sensor with associated task for each section. (C) Scheme representing the followed protocol over time. (D) Imaging of $\mathrm{C} 2 \mathrm{C} 12$ cells treated with the sensor peptide stained with the fluorescent chloropyrimidine label SNAP-Cell TMR-Star from New England BioLabs (TMR, in grey, left panel), of untreated C2C12 stained with the glycogen antibody (in green, middle panel), and of CBM WT infected C2C12 cells stained with the glycogen antibody (in red, right panel). The nuclei were stained with DAPI and are shown in blue. 
glycogen antibody (Fig. 1A).

To overcome the limitations of low efficiency and applicability of exogenous genetic expression such as use of virus infection or transient transfection, we opted for protein transduction which confers to a peptide the ability to cross cell membranes and be imported from the extracellular environment into the cell [21, 22]. In contrast to genebased methods, this technique takes only minutes for peptide import, works on basically all cell types (even primary cells and in vivo) and the degradation of the peptide by the cellular system is probably more rapid than for an exogenous gene [21, 22]. Therefore, the method is ideal for live cell imaging and due to its very short-termed presence in the cell could possibly minimize the bias introduced by the assay.

With this in mind, we designed a construct which contains the CBM of AMPK and a protein transduction domain, and which can be fluorescently labeled (Fig. 1B). More specifically, the construct contained the human AMPK-CBM WT for glycogen binding as well as a pentahistidine tag for purification (Fig. 1B). Additionally, the wellestablished TAT-tag which corresponds to the sequence of the HIV-1 transactivator of transcription protein necessary to enter mammalian cells $[21,22]$ was used as a protein transduction signal. To be able to localize glycogen in cells, the SNAP-tag was chosen: this sequence derived from $\mathrm{O}^{6}$-alkylguanine-DNA alkyltransferase catalyzes the selfattachment of a fluorescently labeled $\mathrm{O}^{6}$-benzylguanine or chloropyrimidine derivative, thereby allowing for in vivo fluorescent labeling [23]. Lastly, after several trials which showed that the peptide got trapped in perimembrane containers after import, we added an INF7-tag (analog of the hemagglutinin 2 peptide) for endosomal escape [24, 25]. The resulting protein after bacterial expression was purified and applied to mammalian cells to visualize their glycogen particles (Fig. 1C).

The preliminary results obtained with this final construct are encouraging as they show a speckle pattern similar to the glycogen pattern seen in untreated cells when these are treated with an anti-glycogen antibody (Fig. 1D, left and middle panels). However, a relatively high variability between peptide batches and between different sets of experiments hindered the progress of the project. Since the level of characterization is quite low at this stage, notably concerning actual co-localization with glycogen, the project still requires a substantial amount of optimization and refinement and was not sufficiently advanced to be included as a whole chapter in this thesis. Nevertheless, in due time, this approach to transiently label glycogen in cells could be added to the many technologies using CBM [20]. Interestingly, in plants, three isolated CBM which each recognized a different type of carbohydrate substrate were used to characterize the constitution of the cell wall [26]: fixed maize coleoptile sections treated with one of the recombinant CBM were stained by immunochemistry which revealed different patterns across the section. If the first sensor is successful, a similar 
approach could be imagined in mammalian cells where CBM recognizing for instance different linkage types of glycogen tagged with separate fluorescent labels could be used simultaneously and allow for detailed glycogen characterization in cells.

Based on the preliminary data, the protein transduction method did not seem to affect the glycogen content in the cells, probably thanks to a very short-term presence in the cell. However, the virus-based expression gives glycogen a different pattern with bigger granules (Fig. 1D, right panel). Therefore, this overexpression shows a potential direct implication of CBM in glycogen metabolism.

\section{CBM in mammals: a role in glycogenesis?}

Although a lot of information has emerged from the study of plant and microbial CBM, the role of CBM in animals seems less well studied. From Chapters $\mathbf{3}$ to 5 of this thesis, our investigations were centered on the AMPK-CBM. In Chapter 4, the PP1 targeting subunit, R6, which also carries a CBM, was under scrutiny. Our observations brought forward an as yet unexplained role for CBM in glycogen synthesis.

In animals, CBM are found in a number of proteins regulating glycogen metabolism such as the glycogen branching and debranching enzymes [27]. In the proteins involved in glycogen regulation, we already mentioned R6 and the related PP1 targeting subunits, as well as AMPK. Laforin is a last example of a CBM-containing enzyme: it interestingly acts as phosphatase with activities both towards glycogen glucose subunits and towards proteins (although the precise targets remain under debate) [9]. For enzymes which catalyze carbohydrates, the advantage a CBM represents in terms of activity was already mentioned [20]. For non-hydrolytic proteins, however, the beneficial aspect of such domain has not been quantified to our knowledge, but one could anticipate a similar increase in glycogen-bound substrate targeting.

In Chapter 4, we report the tremendous increase in glycogen content upon R6 overexpression both in HEK293T and C2C12 cells. In Chapters $\mathbf{3}$ and 5, the cell lines which overexpress the isolated AMPK-CBM also appeared to accumulate more glycogen, even though the data was not presented in either chapter. The pattern of glycogen accumulation seen in cells overexpressing the CBM-WT is different from basal conditions (Fig. 1D, 2A and 2B). Biochemical measurement of glycogen levels confirmed the immunofluorescence data since in this assay, the phenotype associated with AMPK-CBM infection led to significantly increased glycogen levels as compared to non-transfected cells (Fig. 2C). Although the fold change in glycogen content is not as impressive as in R6-overexpressing cells (Chapter 4, 30-fold increase in C2C12), AMPK-CBM overexpression clearly impacts on glycogen regulation.

This phenotype of increased glycogen upon R6 or AMPK-CBM overexpression is not entirely surprising. Previous studies reported an increased glycogen storage upon 
A

Non infected $\mathrm{C} 2 \mathrm{C} 12$ cells
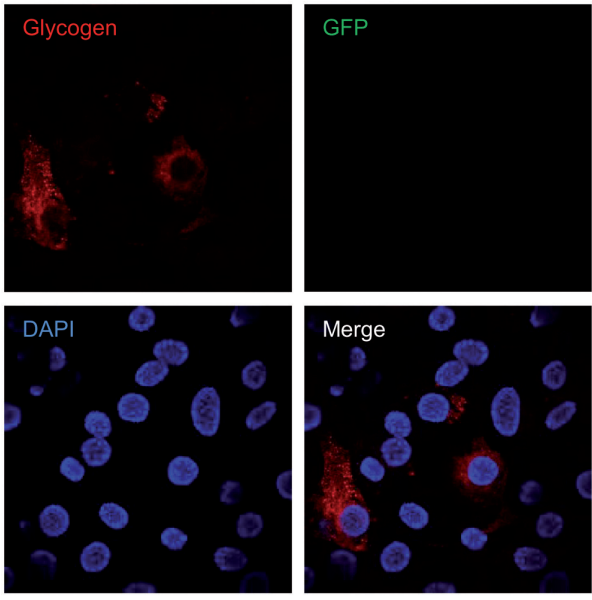

C

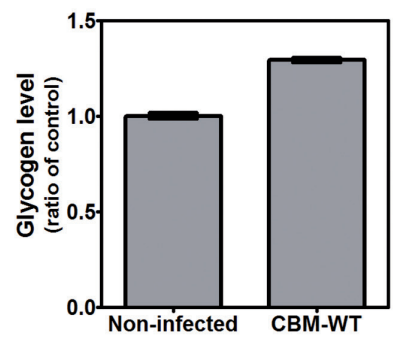

B

AMPK-CBM WT infected C2C12 cells
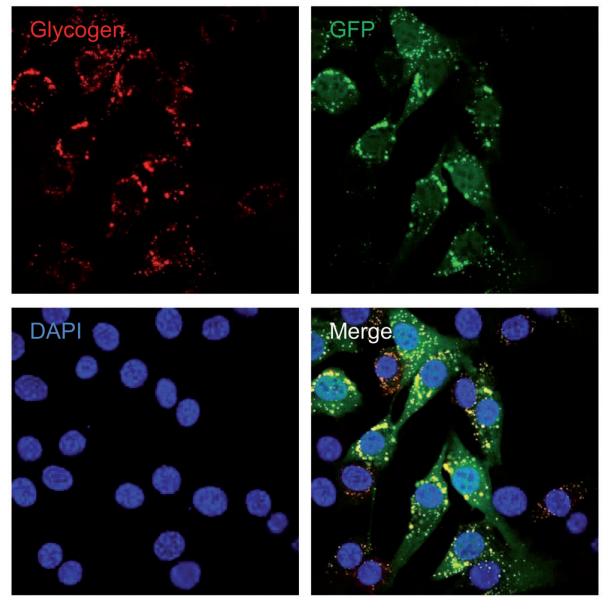

D

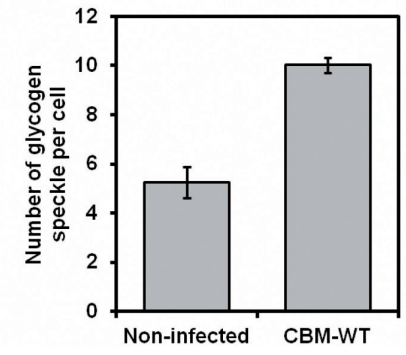

Figure 2. Overexpression of AMPK-CBM WT increases glycogen content.

(A-B) Comparison between the glycogen pattern (fluorescent immunostaining with glycogen antibody shown in red) of non-infected (A) and AMPK-CBM WT (B, GFP-tagged protein shown in green) infected C2C12 cells. DAPI staining of nuclei is represented in blue. (C) Biochemical quantification of glycogen levels in non-infected and AMPK-CBM WT infected C2C12 cells (means \pm SEM). (D) Number of glycogen speckle per cell measure on C2C12 cells either non-infected or infected with AMPK-CBM WT fluorescently (means \pm SEM). Both cell lines were immunostained with a glycogen antibody. Imaging and quantification followed the protocol described for BD pathway in Chapter 5, but for glycogen staining quantification instead of GFP staining.

overexpression of any of several PP1 glycogen targeting subunits (R6, PTG/R5, G ) [28]. Similarly, the first studies that identified the AMPK-CBM reported glycogen inclusions upon overexpression of either the RFP-tagged full trimer or of the GFP-tagged $\beta$-subunit alone $[3,29]$. Both studies compared the pattern of increased glycogen to those obtained either by overexpression of laforin or in hereditary cardiac glycogen diseases even though the data of such disease or even of basal glycogen pattern were not actually 
shown [3, 29]. Yet, accordingly, laforin overexpression leads to a similar strong punctuate pattern that co-localizes with glycogen-bound proteins, even though once again the basal pattern was not shown for comparison [30]. Nevertheless there is a strong correlation between CBM-containing proteins overexpression and glycogen overaccumulation. To our knowledge, this is the first report that an isolated CBM is sufficient to trigger the upregulation of glycogen.

The upregulation of glycogen levels following PP1 glycogen targeting subunit overexpression is primarily explained by increased dephosphorylation of glycogen synthase by PP1 which is known to be an important step in glycogen synthesis upregulation [31, 32]. Similarly, laforin exerts direct roles at glycogen, which although still unclear, may contribute to glycogen accumulation. In contrast, the phenotype associated with the isolated CBM is more surprising as AMPK-CBM alone does not have any catalytic abilities and cannot form a trimer with endogenous AMPK subunits without the other domain of the $\beta$-subunit. Two mechanisms could come at play in explaining the increased glycogen level: either increased number of granules, or increased number of tiers (i.e. increased size) of existing particles. Interestingly, quantification of the number of glycogen speckles per cell using the glycogen antibody suggests that overexpression of AMPK-CBM increases the number of glycogen granules (Fig. 2D). The synthesis of novel glycogen granules depends on the presence of the scaffold protein glycogenin which initiates the first linkages of glucose units. However, glycogenin does not seem to be a rate-limiting enzyme in glycogen synthesis as glycogenin overexpression does not lead to increased glycogen levels $[7,9]$. Therefore it is difficult to imagine how CBM presence could trigger the formation of granules de novo.

In contrast, the immunofluorescence data (Fig. 1D, 2A and 2B) clearly show an increased size of glycogen particles in CBM overexpressing cells, arguing that particle size regulation may also be significant in the overall glycogen increase. In contrast to the de novo formation of granules, a number of hypotheses could be advanced to explain this pattern of increased granule size. For instance, the isolated CBM could compete with endogenous AMPK trimer presence at glycogen and prevent the AMPK-induced inhibition of glycogen synthase. Alternatively, since the interaction between AMPK and R6 reported in Chapter 4 is mediated via the CBM of AMPK, the isolated AMPK-CBM could bring R6 to glycogen and onceagain facilitateglycogen synthasedephosphorylation.

In any case, verifying whether the isolated CBM of laforin and/or of the PP1 glycogen targeting subunit are also able to increase glycogen content would be of great interest. It would suggest that the CBM domains have intrinsic functions in regulating glycogen levels. Interestingly, in such hypothesis, the compound identified in Chapter $\mathbf{5}$ could actually lower glycogen levels through the removal of CBM attachment. 


\section{Future perspectives for compound development}

In this last section of the Discussion, we will focus on the results of Chapter 5, in which we reported the discovery of small molecules able to delocalize AMPK from glycogen and for at least one of them, called 6469172, to activate the kinase. This compound is still in an early stage of development and can be classified as a "hit" at best for now, however, several approaches are possible to further develop and characterize it in the hope that it may lead to a potential drug in the future.

\section{Development based on the identified series of compounds}

In Chapter 5, we describe the discovery of compound 6469172 which binds the human AMPK-CBM and acts on its subcellular localization with a low micromolar activity (IC50 $\sim 9 \mu \mathrm{M}$ ). Additionally, 6469172 treatment increased AMPK phosphorylation at the concentration of $25 \mu \mathrm{M}$, testifying of an acceptable cellular activity and of a very interesting AMPK activating property. Although the link between the two is not fully characterized at this stage, the profile of activity of this compound makes it an interesting starting point for further study.

As described in Chapter 2, AMPK activation could have many implications in a wide set of diseases and is an especially recognized target for metabolic diseases. In Chapter $\mathbf{5}$, we describe an increase in nutrient uptake as well as in acetyl-CoA carboxylase (ACC) phosphorylation which should correlate with increased fatty acid oxidation. Therefore, 6469172 already showed potential for application for insulin resistance, where these two aspects are essential [1]. Obviously, a more thorough testing which involves in vitro and in vivo models will be required to fully characterize the potentiality of this mode of action in insulin resistance treatment.

Despite encouraging data regarding potency in human derived cell lines (Chapter 5), hundreds of compounds need to be synthesized and screened before reaching the status of a final molecule that is fit for use in the clinics [33]. Importantly, the pharmacokinetic properties of the compound have not yet been studied, even though a primary selection for beneficial ADME/tox properties (absorption, distribution, metabolism, excretion / toxicity) by filter of the initial database was made at an early stage of the discovery campaign. A successful compound optimization should be multiparameter and take simultaneously into account potency and drug-like properties [34]. Therefore, a full characterization and potential optimization of the ADME $\backslash$ tox properties of the identified compound is of the utmost importance. Some toxicokinetic studies with isoeugenol found a very rapid absorption and diffusion from the intestinal tract to the organs, but also reported quick metabolism and clearance [35].

In summary, the identified compound 6469172 shows promising results in terms of 
potency and cellular activity for a first generation hit molecule. Nevertheless, a long way stays ahead for full optimization of this molecule before it can be considered a true lead molecule.

\section{Search for a new scaffold using the identified hit molecule as proof-of-concept}

During the drug development process, several compound series should be followed to increase the chances that at least one will reach the desired goal [33]. In Chapter 5, two scaffolds of isoeugenol derivatives have been described. Four additional compound types were identified during the screening that have not been presented in the current work, as only one compound per type was found positive. It is still appropriate to stress that negative data are also of importance, as they add to the complete description of the structure-activity relationship and knowledge of inactives in a particular compound family is as valuable as structural data on the actives. Nevertheless, should additional structures be found, the identified series of compounds could be used as proof-ofconcept and positive controls in a new screening assay.

Besides virtual screening, for which we already discussed the implications following the first compound identification (see Chapter 5, discussion), some initiatives emerged in the recent years to bring high-throughput screening within reach of academic research: for instance the European Lead Factory (ELF) offers access to a screening library of 500,000 compounds to 24 selected projects a year [36]. Our simple two-step in vitro screening protocol has offered proof-of-principle for its efficacy in identifying hit molecules delocalizing AMPK from glycogen and could therefore also be used in principle for fast and relatively cheap high-throughput screening. However, additional criteria have to be taken into account when upscaling an assay from an 800-compound library to one containing several hundreds of thousands: notably the assay quality and reproducibility are very stringent criteria [33]. Moreover supernatant transfers are not allowed in high-throughput screenings (ELF, personal communication), indicating that the readout of the initial competition assay would have to be changed. An alternative approach could be based on the AlphaScreen (Perkin Elmer) which is routinely used in high-throughput screenings for its high sensitivity [37]. In that setting, biotinylated $\beta$-cyclodextrin would be recognized by the streptavidin coated donor beads, while the tagged AMPK-CBM would be recognized by antibodies linked to the acceptor beads. When the CBM interacts with the carbohydrate, the transmission from donor to acceptor bead would be high, whereas the signal would be disrupted by addition of a competitive small molecule. Nevertheless, the identified screening steps could be used in a high-throughput screening after some adjustments.

Interestingly, other classes of carbohydrate-binding protein are targets of drug design projects. Specifically, lectin targeting is being pursued because of their role in 
cell adhesion and recognition [38]. Since carbohydrates constitute poor drugs due to low absorption and high metabolism, glycomimetic drugs have been developed based on known ligands. A similar strategy may yield interesting results in the case of AMPKCBM.

In summary, additional approaches are available to identify novel series of compounds able to disrupt AMPK-glycogen attachment. However they will hopefully not be needed if the identified compounds in Chapter $\mathbf{5}$ show promising pharmacokinetics and potency results during optimization.

\section{Concluding remarks}

AMPK-glycogen interplay stays a complicated matter with interesting roles in health and disease. This thesis contributed to a better understanding of the regulation and roles of AMPK in the synthesis and consumption of glycogen. In line with these results, the identified compounds, 6469172 and 6456019, could potentially be further developed into lead compounds that may be applied in the pharmacotherapy of metabolic diseases or other AMPK-related fields. Even though a long way stays ahead for their development, promising results have been unveiled which can hopefully one day be transmitted to the clinics. 


\section{References}

1. Samuel, V.T. and Shulman, G.I., Mechanisms for insulin resistance: common threads and missing links. Cell, 2012. 148(5): p. 852-871.

2. Hegarty, B.D., Turner, N., Cooney, G.J. and Kraegen, E.W., Insulin resistance and fuel homeostasis: the role of AMP-activated protein kinase. Acta Physiol (Oxf), 2009. 196(1): p. 129-145.

3. Hudson, E.R., Pan, D.A., James, J., Lucocq, J.M., Hawley, S.A., Green, K.A., Baba, O., Terashima, T. and Hardie, D.G., A novel domain in AMP-activated protein kinase causes glycogen storage bodies similar to those seen in hereditary cardiac arrhythmias. Current biology : CB, 2003. 13(10): p. 861-866.

4. Polekhina, G., Gupta, A., van Denderen, B.J., Feil, S.C., Kemp, B.E., Stapleton, D. and Parker, M.W., Structural basis for glycogen recognition by AMP-activated protein kinase. Structure, 2005. 13(10): p. 1453-1462.

5. Smith, C., Drug target validation: Hitting the target. Nature, 2003. 422(6929): p. 341, 343, 345 passim.

6. Tsatsoulis, A., Mantzaris, M.D., Bellou, S. and Andrikoula, M., Insulin resistance: an adaptive mechanism becomes maladaptive in the current environment - an evolutionary perspective. Metabolism, 2013. 62(5): p. 622-633.

7. Graham, T.E., Yuan, Z., Hill, A.K. and Wilson, R.J., The regulation of muscle glycogen: the granule and its proteins. Acta Physiol (Oxf), 2010. 199(4): p. 489-498.

8. Prats, C., Gomez-Cabello, A. and Hansen, A.V., Intracellular compartmentalization of skeletal muscle glycogen metabolism and insulin signalling. Exp Physiol, 2011. 96(4): p. 385-390.

9. Roach, P.J., Depaoli-Roach, A.A., Hurley, T.D. and Tagliabracci, V.S., Glycogen and its metabolism: some new developments and old themes. Biochem J, 2012. 441(3): p. 763-787.

10. Prats, C., Cadefau, J.A., Cusso, R., Qvortrup, K., Nielsen, J.N., Wojtaszewski, J.F., Hardie, D.G., Stewart, G., Hansen, B.F. and Ploug, T., Phosphorylation-dependent translocation of glycogen synthase to a novel structure during glycogen resynthesis. J Biol Chem, 2005. 280(24): p. 23165-23172.

11. Jorgensen, S.B., Nielsen, J.N., Birk, J.B., Olsen, G.S., Viollet, B., Andreelli, F., Schjerling, P., Vaulont, S., Hardie, D.G., Hansen, B.F., Richter, E.A. and Wojtaszewski, J.F., The alpha2-5'AMP-activated protein kinase is a site 2 glycogen synthase kinase in skeletal muscle and is responsive to glucose loading. Diabetes, 2004. 53(12): p. 30743081.

12. Hunter, R.W., Treebak, J.T., Wojtaszewski, J.F. and Sakamoto, K., Molecular mechanism by which AMP-activated protein kinase activation promotes glycogen accumulation in muscle. Diabetes, 2011. 60(3): p. 766-774.
13. McBride, A., Ghilagaber, S., Nikolaev, A. and Hardie, D.G., The glycogen-binding domain on the AMPK beta subunit allows the kinase to act as a glycogen sensor. Cell Metab, 2009. 9(1): p. 2334.

14. Koay, A., Woodcroft, B., Petrie, E.J., Yue, H., Emanuelle, S., Bieri, M., Bailey, M.F., Hargreaves, M., Park, J.T., Park, K.H., Ralph, S., Neumann, D. Stapleton, D. and Gooley, P.R., AMPK beta subunits display isoform specific affinities for carbohydrates. FEBS Lett, 2010. 584(15): p. 3499 3503.

15. Dela, F., Prats, C. and Helge, J.W., Exercise interventions to prevent and manage type 2 diabetes: physiological mechanisms. Med Sport Sci, 2014. 60: p. 36-47.

16. Kim, M. and Tian, R., Targeting AMPK for cardiac protection: opportunities and challenges. J Mol Cell Cardiol, 2011. 51(4): p. 548-553.

17. Roma-Mateo, C., Solaz-Fuster Mdel, C., GimenoAlcaniz, J.V., Dukhande, V.V., Donderis, J., Worby, C.A., Marina, A., Criado, O., Koller, A., Rodriguez De Cordoba, S., Gentry, M.S. and Sanz, P., Laforin, a dual-specificity phosphatase involved in Lafora disease, is phosphorylated at Ser25 by AMP-activated protein kinase. Biochem J, 2011. 439(2): p. 265-275.

18. Shoseyov, O., Shani, Z. and Levy, I., Carbohydrate binding modules: biochemical properties and novel applications. Microbiol Mol Biol Rev, 2006. 70(2): p. 283-295.

19. Guillen, D., Sanchez, S. and Rodriguez-Sanoja, R., Carbohydrate-binding domains: multiplicity of biological roles. Appl Microbiol Biotechnol, 2010. 85(5): p. 1241-1249.

20. Oliveira, C., Carvalho, V., Domingues, L. and Gama, F.M., Recombinant CBM-fusion technology - Applications overview. Biotechnol Adv, 2015. 33(3-4): p. 358-369.

21. Brooks, H., Lebleu, B. and Vives, E., Tat peptidemediated cellular delivery: back to basics. Adv Drug Deliv Rev, 2005. 57(4): p. 559-577.

22. van den Berg, A. and Dowdy, S.F., Protein transduction domain delivery of therapeutic macromolecules. Curr Opin Biotechnol, 2011. 22(6): p. 888-893.

23. Crivat, G. and Taraska, J.W., Imaging proteins inside cells with fluorescent tags. Trends Biotechnol, 2012. 30(1): p. 8-16.

24. Liou, J.S., Liu, B.R., Martin, A.L., Huang, Y.W., Chiang, H.J. and Lee, H.J., Protein transduction in human cells is enhanced by cell-penetrating peptides fused with an endosomolytic HA2 sequence. Peptides, 2012. 37(2): p. 273-284.

25. Wadia, J.S., Stan, R.V. and Dowdy, S.F., Transducible TAT-HA fusogenic peptide enhances escape of TAT-fusion proteins after lipid raft macropinocytosis. Nat Med, 2004. 10(3): p. 310-315. 
26. McCartney, L., Gilbert, H.J., Bolam, D.N., Boraston, A.B. and Knox, J.P., Glycoside hydrolase carbohydrate-binding modules as molecular probes for the analysis of plant cell wall polymers. Anal Biochem, 2004. 326(1): p. 49-54.

27. Diaz, A., Martinez-Pons, C., Fita, I., Ferrer, J.C. and Guinovart, J.J., Processivity and subcellular localization of glycogen synthase depend on a non-catalytic high affinity glycogen-binding site. J Biol Chem, 2011. 286(21): p. 18505-18514.

28. Worby, C.A., Gentry, M.S. and Dixon, J.E., Malin decreases glycogen accumulation by promoting the degradation of protein targeting to glycogen (PTG). J Biol Chem, 2008. 283(7): p. 4069-4076.

29. Polekhina, G., Gupta, A., Michell, B.J., van Denderen, B., Murthy, S., Feil, S.C., Jennings, I.G., Campbell, D.J., Witters, L.A., Parker, M.W., Kemp, B.E. and Stapleton, D., AMPK beta subunit targets metabolic stress sensing to glycogen. Current biology : CB, 2003. 13(10): p. 867-871.

30. Wang, J., Stuckey, J.A., Wishart, M.J. and Dixon, J.E., A unique carbohydrate binding domain targets the lafora disease phosphatase to glycogen. J Biol Chem, 2002. 277(4): p. 2377-2380.

31. Gasa, R., Jensen, P.B., Berman, H.K., Brady, M.J., DePaoli-Roach, A.A. and Newgard, C.B., Distinctive regulatory and metabolic properties of glycogen-targeting subunits of protein phosphatase-1 (PTG, GL, GM/RGI) expressed in hepatocytes. J Biol Chem, 2000. 275(34): p. 2639626403.
32. Greenberg, C.C., Meredith, K.N., Yan, L. and Brady, M.J., Protein targeting to glycogen overexpression results in the specific enhancement of glycogen storage in 3T3-L1 adipocytes. J Biol Chem, 2003. 278(33): p. 30835-30842.

33. Hughes, J.P., Rees, S., Kalindjian, S.B. and Philpott, K.L., Principles of early drug discovery. Br J Pharmacol, 2011. 162(6): p. 1239-1249.

34. Lusher, S.J., McGuire, R., Azevedo, R., Boiten, J.W., van Schaik, R.C. and de Vlieg, J., A molecular informatics view on best practice in multi-parameter compound optimization. Drug discovery today, 2011. 16(13-14): p. 555-568.

35. Hong, S.P., Fuciarelli, A.F., Johnson, J.D., Graves, S.W., Bates, D.J., Waidyanatha, S. and Smith, C.S., Toxicokinetics of methyleugenol in F344 rats and B6C3F(1) mice. Xenobiotica, 2013. 43(3): p. 293-302.

36. Mullard, A., European lead factory opens for business. Nat Rev Drug Discov, 2013. 12(3): p. 173-175.

37. Eglen, R.M., Reisine, T., Roby, P., Rouleau, N., Illy, C., Bosse, R. and Bielefeld, M., The use of AlphaScreen technology in HTS: current status. Curr Chem Genomics, 2008. 1: p. 2-10.

38. Ernst, B. and Magnani, J.L., From carbohydrate leads to glycomimetic drugs. Nat Rev Drug Discov, 2009. 8(8): p. 661-677. 



\section{Appendices}

List of abbreviations

Summary

Samenvatting

Résumé

Valorization

Curriculum vitae Acknowledgments 



\section{List of abbreviations}

[PP]2-InsP4

ACC

ADME/tox

AICAR

AMPK

CaMKK $\beta$ (or CaMKK2)

CBM

CBS

Cidea

CRTC

eEF2

ELF

ERK

GB8

GP

GS

HDAC

I, S, P, L

IC50

iFCS

IP

KD

$\mathrm{K}_{\mathrm{D}}$

$\log \mathrm{P}$

LKB1

MAPK

mTOR

MTT

NLRP3

NMR

PGC1 $\alpha$

PP

PP1

PPI

R6

RAPTOR

Reg1 bis-diphosphoinositol tetrakisphosphate

acetyl-coenzyme A carboxylase

absorption, distribution, metabolism, excretion / toxicity

5-amino-4-imidazolecarboxamide ribonucleoside

AMP-activated protein kinase (AMPK $\beta$ : $\beta$-subunit of AMPK)

calcium/calmodulin-dependent protein kinase kinase $\beta$ (or 2)

carbohydrate-binding module (CBM $\beta 1$ and CBM $\beta 2$ : CBM of AMPK- $\beta 1$ and $-\beta 2$ subunit, respectively)

cystathione $\beta$-synthase

cell death-inducing DNA fragmentation factor $\alpha$-like effector A

cyclic AMP response element binding protein (CREB)-regulated transcription coactivators

eukaryotic elongation factor 2

European Lead Factory

extracellular signal-regulated kinase

generalized Born model 8

glycogen phosphorylase

glycogen synthase

class II histone deacetylases

input, supernatant, pellet and Laemmli fractions of the $\beta$-cyclodextrin assay, respectively (see Chapter 3 )

half maximal inhibitory concentrations

heat-inactivated fetal calf serum

immunoprecipitation

kinase deficient

equilibrium dissociation constants

logarithm of the partition coefficient

liver kinase B1

mitogen-activated protein kinases

mammalian target of rapamycin

3-(4,5-dimethylthiazol-2-yl)-2,5-diphenyl tetrazolium bromide

nucleotide binding and oligomerization (NOD)-like receptor family pyrin domain containing 3

nuclear magnetic resonance

peroxisome proliferator-activated receptor gamma coactivator $1 \alpha$

protein phosphatases

protein phosphatase type 1

protein-protein interaction

PP1-glycogen-targeting subunit (PPP1R3D)

regulatory-associated protein of mTOR

yeast targeting subunit of PP1 
RNS

ROS

SIRT1

Snf1

SnRK1

SREBP1

T148A

T148D

TAK1

TBC1D1

TSC2

ULK1

UPR

VLS

Y2H

$\alpha$-RIM

$\alpha$-SNAP

$\beta$-CD

$\Delta \mathrm{G}_{\text {cal. }}$

$\Delta \mathrm{G}_{\text {exp. }}$ reactive nitrogen species

reactive oxygen species

sirtuin 1

sucrose-non-fermenting 1

Snf1-related protein kinase 1

sterol regulatory element-binding protein 1

phosphorylation-resistant mutation of AMPK $\beta$ T148 residue to alanine

phosphorylation-mimicking mutation of AMPK $\beta$ T148 residue to aspartate

transforming growth factor $\beta$-activated protein kinase

TRE2/BUB2/CDC16 domain family member 1

tuberous sclerosis 2

unc-51-like kinase 1

unfolded protein response

vitrual ligand screening

yeast two-hybrid

$\alpha$-regulatory subunit interacting motif

soluble $\mathrm{N}$-ethylmalemide sensitive factor attachment protein

$\beta$-cyclodextrin

calculated binding free energy

experimental binding free energy 


\section{Summary}

At the whole body and cellular levels, the balance between energy expenditure and storage is of utmost importance to adapt to a changing environment. The AMP-activated protein kinase (AMPK) is a major regulator of energy balance, which, once activated, increases energy production and down-regulates its consumption. Because the effects of AMPK mimics those of exercise and calorie restriction, the pharmacological activation of AMPK has been proposed as a potential treatment of type 2 diabetes, a disease associated with poor lifestyle.

Beyond this well-established role in diabetes, AMPK has emerged over the years as an important player in a great number of disorders such as heart failure and atherosclerosis (Chapter 2). However, despite a continuous interest, efficient and safe targeting strategies of AMPK are still not available. A possible approach to overcome the current shortcomings is to use virtual screening based on the many known structures of AMPK protein and its ligands.

An interesting feature of AMPK regulation is the ability to bind to glycogen: although AMPK does not exert any catalytic activity towards saccharides, this binding, enabled by the presence of a carbohydrate-binding module (CBM) on the $\beta$-subunit of AMPK, influences kinase activity. In this thesis, the aim was to further clarify the interdependence between AMPK and glycogen, in particular in the context of nutrient metabolism.

Firstly, a regulation of AMPK binding to glycogen was identified in the autophosphorylation of AMPK at the $\beta$-Thr148 site (Chapter 3). Under basal condition, little Thr148 phosphorylation was found whereas cellular stresses such as glucose deprivation or mitochondrial inhibition increased both AMPK activity and autophosphorylation. The phosphorylation-mimicking mutation of Thr148 to Asp prevented the binding of AMPK trimer to glycogen in vitro and in cells, which indicates that Thr148 autophosphorylation prevents AMPK binding to glycogen. This regulation may play a role in glycogen metabolism as the Thr148 mutation prevented the inhibition of glycogen resynthesis induced by AMPK activators.

Secondly, this same autophosphorylation was implicated in AMPK binding with a protein partner, the protein phosphatase 1 targeting subunit, named R6, which also binds glycogen via a CBM (Chapter 4). R6 and AMPK were found to interact through the substrate binding motif of the former and the $\beta$-subunit of the latter. Under low glucose and more generally glycogen-degrading conditions, AMPK-R6 interaction was enhanced. The binding was even further increased by treatment with an AMPK activator which correlated with upregulated Thr148 autophosphorylation. Altogether, the data suggested that AMPK-R6 interaction is direct, not mediated by glycogen. This 
interaction may be relevant in protein phosphatase 1-induced dephosphorylation of AMPK upon refeeding-like conditions.

Thirdly, considering the interplay between AMPK and glycogen, the design of small molecules able to disrupt this interaction could offer a novel strategy to target AMPK (Chapter 5). Our screening protocol, composed of a virtual screening and a two-step in vitro screen, led to the identification of a cluster of structurally-related compounds. The two most active compounds, designated 6469172 and 6456019 , changed the AMPK-CBM localization in cells in a dose-dependent manner, directly bound to the isolated CBM, and disrupted the interaction between AMPK and another glycogenbound protein, i.e. glycogen synthase. Additionally, the compound 6469172 enhanced AMPK activity in different cell lines and increased glucose and fatty acid uptake which is a well-established effect of AMPK activators.

In conclusion, this thesis offers major advances in understanding the interplay between AMPK and glycogen, and presents a new tool to further explore this peculiar interdependence. 


\section{Samenvatting}

De balans tussen energieverbruik en energieopslag is van groot belang voor zowel het hele lichaam als voor individuele cellen en weefsels. Dit geldt met name wanneer er sprake is van aanpassing aan een veranderende omgeving. Het zogenaamde AMPgeactiveerde eiwitkinase (AMPK) is een belangrijke regulator van deze energiebalans. Activatie van AMPK zorgt voor een verhoging van de energieproductie en tegelijkertijd voor een beperking van het energieverbruik. De effecten die AMPK heeft lijken daarmee op die van inspanning en op die van een beperking van voedselinname. Er is voorgesteld om met behulp van farmacologische middelen AMPK te activeren als een mogelijke nieuwe behandeling van type 2 diabetes. Bij deze (meest voorkomende) vorm van diabetes is het hormoon insuline onvoldoende in staat om de opname van suikers door de weefsels - en daarmee een adequate energieproductie - te stimuleren. Deze rol zou nu overgenomen kunnen worden door activatie van AMPK.

Naast deze goed-omschreven rol in diabetes, is AMPK in de laatste jaren ook in de belangstelling gekomen als een belangrijk eiwit voor een aantal andere ziektes en aandoeningen, waaronder hartfalen en aderverkalking ofwel atherosclerose (Hoofdstuk 2). Ondanks deze groeiende belangstelling beschikken we nog niet over een efficiënte en veilige manier om farmacologische stoffen te ontwikkelen die AMPK kunnen activeren. Een recent beschikbaar gekomen mogelijke manier waarmee dit zou kunnen is door gebruik te maken van zogenaamde virtuele selectiemethoden. Daarbij wordt gebruik gemaakt van bekende driedimensionale (3D) eiwitstructuren van AMPK en van zijn bindingspartners of substraten.

Een interessante eigenschap van AMPK is het vermogen om aan glycogeen te binden. Glycogeen is de cellulaire opslagvorm van opgenomen suikers (glucose). Hoewel AMPK geen enzymatische activiteit bezit ten opzichte van glycogeen, beïnvloedt binding eraan wel de kinase activiteit van het AMPK. Deze binding wordt gemedieerd door een zogenaamde koolhydraat-bindende-module (CBM) binnen de $\beta$-subeenheid van AMPK. In dit proefschrift hebben wij ons gericht op een nadere beschrijving van het verband tussen AMPK en glycogeen, in het bijzonder in de context van het metabolisme van voedingsstoffen zoals suikers (glucose) en vetten (vetzuren).

Ten eerste beschrijven we de regulatie van AMPK binding aan glycogeen door autofosforylering van AMPK van het aminozuur threonine148 (Hoofdstuk 3). In rust wordt er nauwelijks fosforylering waargenomen van threonine148, terwijl tijdens stress - zoals bij afwezigheid van glucose of bij gebruik van remmers van de oxidatieve fosforylering - de activiteit van AMPK (en de autofosforylering) juist toenemen. Door threonine148 te veranderen in asparaginezuur148 wordt fosforylering geïmiteerd maar deze verandering verhindert de binding van AMPK aan glycogeen, zowel in vitro 
als ook in cellen. Dit betekent dat autofosforylering van threonine148 de binding van AMPK aan glycogeen helpt te voorkomen. Deze vorm van regulatie speelt mogelijk een rol in het glycogeenmetabolisme omdat in aanwezigheid van AMPK activatoren de verandering van threonine148 de glycogeensynthese kan remmen.

Ten tweede is deze zelfde autofosforylering betrokken bij de binding van AMPK aan een ander molecuul: de zogenaamde eiwit fosfatase 1 targeting subeenheid, ook wel R6 genoemd. Ook R6 bevat een eerder genoemde CBM module (Hoofdstuk 4). R6 en AMPK bleken een interactie aan te gaan via het substraat-bindend domein van R6 en de $\beta$-subeenheid van AMPK. Wanneer glucose schaars is of meer algemeen wanneer glycogeen wordt afgebroken, wordt de interactie tussen R6 en AMPK bevorderd. Deze binding werd zelfs verder bevorderd door toevoeging van een AMPK activator die leidt tot een toename in de autofosforylering van threonine148. Samengevat suggereren onze onderzoeksresultaten dat de interactie tussen AMPK en R6 direct is, zonder bijdrage van glycogeen. Deze interactie is mogelijk relevant bij eiwit fosfatase 1-geinduceerde defosforylering van AMPK.

Ten derde, zou de ontwikkeling van kleine moleculen die de interactie tussen AMPK en glycogeen remmen een mogelijkheid kunnen bieden om de activiteit van AMPK farmacologisch te beïnvloeden (Hoofdstuk 5). Het door ons gepresenteerde protocol voor selectie van kleine moleculen, gebaseerd op een virtuele selectie en daarna een twee-staps in vitro selectie, heeft geleid tot de ontdekking van een serie van soortgelijke kleine moleculen. De beste twee stoffen, aangeduid met 6469172 en 6456019, zijn in staat om, afhankelijk van de dosis, de lokalisatie van AMPK in levende cellen te beïnvloeden, om direct te binden aan de CBM subeenheid en om de interactie van AMPK met nog een ander glycogeen-gebonden eiwit, het glycogeen synthase, te verhinderen. Bovendien bevordert 6469172 de AMPK activiteit in verschillende cellijnen en verhoogt het de opname van glucose en vetzuren, een effect dat ook bekend is van reeds ontdekte AMPK-activatoren.

Samenvattend beschrijft dit proefschrift een aantal grote vorderingen in ons begrip van het samenspel tussen AMPK en glycogeen en daarnaast wordt een nieuwe aanpak gepresenteerd die gebruikt kan worden om dit samenspel verder te onderzoeken. 


\section{Résumé}

Au niveau corporel comme au niveau cellulaire, la balance entre dépense et stockage d'énergie est de la plus haute importance pour pouvoir s'adapter aux conditions changeantes de l'environnement. La protéine kinase activée par l'AMP (AMP-activated protein kinase, AMPK) est l'un des régulateurs principaux de cette balance énergétique : une fois activée, AMPK favorise les mécanismes de production d'énergie tout en diminuant ceux liés à sa consommation. Parce que ces effets générés par AMPK sont similaires à ceux liés au sport et à la restriction calorique, l'activation par voie pharmacologique d'AMPK est considérée comme l'une des approches possibles pour traiter le diabète de type 2 , c'est-à-dire la forme associée à une mauvaise hygiène de vie et au vieillissement.

Au-delà de ce rôle bien connu, AMPK est aussi l'un des acteurs centraux de beaucoup d'autres maladies, comme l'insuffisance cardiaque par exemple (Chapitre 2). Cependant, malgré l'intérêt certain qu'AMPK génère, aucun agent pharmacologique sûr et efficace pour cibler AMPK n'a été reporté jusqu'à présent. Pour remédier à ce manque, une approche possible serait l'utilisation de méthodes de screening virtuel qui s'appuieraient sur les nombreuses structures connues de la protéine AMPK aussi bien que de ses ligands.

La régulation d'AMPK est complexe mais l'un des éléments intéressants est sa capacité à se lier au glycogène, la principale forme de stockage glucidique des cellules : bien qu'AMPK n'ait pas d'activité catalytique sur les saccharides, cette interaction, permise par la présence d'un domaine d'attachement aux glucides (carbohydratebinding module, CBM) dans la sous-unité $\beta$ d'AMPK, influence l'activité de la kinase. L'objectif de cette thèse est de clarifier l'interdépendance entre AMPK et le glycogène, en particulier dans le contexte du métabolisme des nutriments.

Premièrement, le site d'autophosphorylation $\beta$-Thr148 est identifié comme jouant un rôle dans la régulation de l'interaction AMPK-glycogène (Chapitre 3). Dans des conditions basales, le niveau de phosphorylation de Thr-148 est peu élevé tandis que les situations de stress cellulaire, comme par exemple l'absence de glucose ou l'inhibition de la mitochondrie, augmentent à la fois l'activité d'AMPK et son autophosphorylation. La mutation de Thr-148 en Asp qui imite la phosphorylation de ce résidu empêche AMPK d'interagir avec le glycogène à la fois in vitro et en cellule, ce qui indique que l'autophosphorylation de Thr-148 empêche AMPK de se lier au glycogène. Ce mécanisme de régulation semble intervenir dans le métabolisme du glycogène puisque la mutation de Thr-148 prévient l'inhibition de la resynthèse de glycogène initiée par les activateurs d'AMPK.

Deuxièmement, le même site d'autophosphorylation est impliqué dans l'interaction 
entre AMPK et une autre protéine, l'une des sous-unités régulatrices de la phosphoprotéine phosphatase 1 (PP1) appelée R6 (Chapitre 4). Comme AMPK, R6 contient un CBM et est capable de se lier au glycogène. Néanmoins, nos données suggèrent une interaction directe entre AMPK et R6 et non via l'intermédiaire du glycogène. R6 et AMPK interagissent via le motif d'interaction du premier et la sousunité $\beta$ du deuxième. Lorsque le niveau de glucose est bas et plus généralement lors de la dégradation du glycogène, l'interaction entre R6 etAMPKaugmente. Leur attachement s'accentue encore d'avantage en cas de traitement avec un activateur d'AMPK en corrélation avec un plus haut niveau d'autophosphorylation de Thr-148. Cette interaction pourrait avoir des conséquences sur la déphosphorylation et l'inactivation d’AMPK permise par PP1 lors de la prise de repas.

Troisièmement, étant donnée l'interdépendance entre AMPK et le glycogène, le design d'une petite molécule chimique capable de perturber cette interaction pourrait se traduire en une stratégie intéressante pour cibler AMPK de manière pharmacologique (Chapitre 5). Notre protocole de screening composé d'une partie virtuelle suivie de deux étapes in vitro, a permis l'identification d'un groupe de molécules avec une structure similaire. Les deux molécules les plus actives, appelées 6469172 et 6456019 , changent la localisation subcellulaire de AMPK-CBM de manière dose-dépendante, interagissent directement avec le CBM d'AMPK et perturbent l'interaction entre AMPK et une autre protéine liée au glycogène, la glycogène synthase. Qui plus est, la molécule 6469172 accroît l'activité d'AMPK dans différentes lignées cellulaires et provoque l'absorption de glucose et d'acide gras par les cellules, un effet bien connu des activateurs d'AMPK.

En conclusion, cette thèse offre des avancées majeures pour la compréhension de l'inter-régulation entre AMPK et le glycogène et présente un nouvel outil pour continuer à explorer cette relation particulière. 


\section{Valorization}

Beyond the scientific relevance of the thesis issued herein, the presented work describes an area of research that could be translated into a socially and economically relevant product. In Chapter 5, the disclosed cluster of small, drug-like molecules which act on the energy sensor AMPK could lead in the future to a new drug of interest in a number of diseases.

\section{A new drug to target AMPK in an innovative manner}

\section{Relevance \& Target groups}

As described in Chapter 2, AMPK is involved in a wide number of diseases, from cardiac diseases to cancer through diabetes. Over the years, this protein has emerged as a potential drug target in many of these fields and the area of research keeps expanding as novel AMPK downstream effects unravel. The most established domain in which AMPK beneficial targeting is known is type 2 diabetes. In contrast to type 1 diabetes, type 2 diabetes is associated to obesity, physical inactivity, poor diet and age. Due to the change in lifestyle induced by worldwide economic development, more and more people are at risk of developing this condition [1]. According to the most recent estimations of the International Diabetes Federation, 382 millions of people suffer from diabetes worldwide and this number is predicted to have risen by 55\% in 2035 if trends keep as they are. Beyond the direct risk associated with the disease, diabetes leads to many more health complications such as cardiovascular diseases and kidney failure. Logically, the costs in healthcare created by diabetes are enormous: as a consequence of undiagnosed diabetes alone, the healthcare costs in the USA were estimated to USD 18 billion for one year [1].

If only for its beneficial role in treatment of this metabolic disorder, AMPK is therefore well worth studying. Moreover, in the past decades, a few proteins in specific cellular pathways have emerged as responsible for age-related disease occurrence [2, 3]. To clarify, the mutation of these proteins in animal models can prolong their healthy lifespan significantly. For some of these targets such as those related to insulin/IGF1, certain mutations correlate with longevity in different human cohorts [2]. Although AMPK did not yet reach such a level of characterization, there is evidence to show that AMPK activation may also potentiate an increase of healthy lifespan [3].

Even though AMPK is involved in many pathways, studies using indirect or direct activators showed mostly beneficial effects without side-effects in a wide array of diseases [4]. Only in very specific cases of the fields of cancer and neurodegenerative disorders, the activation of AMPK sometimes led to worsening of the symptoms. Therefore, the targeting of AMPK is considered as generally safe with only cautions to 
be taken under some conditions.

In summary, considering the high occurrence of diseases related to ageing or "modern" lifestyle that could benefit from AMPK activity, the development of bioactive compounds that target AMPK is of obvious relevance, both from social and economic points of view.

\section{Product \& Innovation}

Several methods to directly or indirectly activate AMPK with drug-like molecules have been described [5]. For direct targeting, three different methods exist that activate the protein through action on different aspects of AMPK regulation. In each of these categories, a high number of studies have led to the identification of many compounds with different structures. Unfortunately, to date, none of these molecules have reached the market, generally due to off-target effects or to poor absorption in the body. With regards to indirect activation of AMPK, different types of molecules mimic an energy stress within the cell they reach, thereby activating AMPK. Among these, several natural products and nutraceuticals, such as resveratrol or curcumin, were shown to activate AMPK [6]. More importantly, the most widely prescribed drug, metformin, also activates AMPK in an indirect manner [7]. This treatment against type 2 diabetes exerts its beneficial effects partly through AMPK. However this drug as well as all indirect AMPK activators has many other targets than AMPK. Therefore the search for a safe and efficient strategy to directly target the protein is still on-going.

In Chapter 5, we describe the identification of novel molecules that target AMPK in an innovative manner. Our primary aim in designing these compounds was not to directly modulate AMPK activity but rather AMPK effects: instead of influencing AMPK activity as other AMPK activators do, our compounds were selected for their ability to modify AMPK localization within the cell, and bring it away from the cellular store of glucose, glycogen. This change of localization should influence the protein partners that AMPK can find and thereby alters the outcome of AMPK activity. Unexpectedly, the compound, named 6469172, which had the strongest ability to change AMPK localization, also modulates AMPK activity. The precise mechanism that triggers this increase in activity is still unexplained at this stage, but still provoked glucose uptake in cells treated with 6469172. Therefore this compound shows promises for the treatment of type 2 diabetes where glucose needs to be taken up from the blood into cellular reserves.

In summary, the newly identified compound, 6469172, is the basis on which a new drug targeting AMPK could be developed. The mechanism of action which targets AMPK localization rather than directly its activity makes it an innovation in the field of AMPK targeting. Considering on one hand the interest that AMPK activators trigger for 
their beneficial roles in many diseases and on the other hand the current lack of an efficient strategy to take advantage of AMPK effects, the compound 6469172 shows interesting potential for further development into a marketed product.

\section{Schedule \& Implementation}

Despite its great interest for future applications, the compound 6469172 is still in a very early stage of a drug development. The data presented within this thesis establish the ability of 6469172 to change AMPK localization and to bind directly to one of the domain of this protein. Furthermore, the first tests show the change of AMPK activity induced by treatment with 6469172 and the resulting increase in glucose and fatty acid uptake. Although these data are encouraging, they are far from sufficient. As explained in Chapter 6, even though bioinformatics tools were used to filter out molecules that were unlikely to behave as drugs, many tests still need to be conducted to certify that our newly-found molecule meets the requirements regarding absorption, distribution, metabolism and excretion (ADME) once in the body [8]. Strict criteria regarding toxicity have also to be adhered to. Moreover, further levels of characterization, both in vivo and in vitro, have to be followed to assess the benefits that this molecule can trigger in disease conditions. Finally, the compound 6469172 together with other structurallyrelated ones identified herein will serve to document structure-activity relationship studies, which will lead to the definition of another chemical structure with optimal characteristics in both ADME/toxicity and potency tests. This development phase is called hit-to-lead and is followed by lead optimization. After these stages, the final compounds enter clinical trials, which is again a very long and costly process.

Therefore, a long road still awaits 6469172 before the concept developed with this molecule can be translated into a commercial product. Generally, from the initial idea to the market launch, the development of a drug takes 12-15 years and costs more than one billion USD [8]. Our initial work brought us from initial idea to the beginning of the hit-to-lead phase, but the main costs remain ahead with the clinical trials to come. Even though the phases of early drug development are progressively taken over by academic groups or academic drug development centers, clinical trials remains the exclusive field of established pharmaceutical companies or their spin-off. In the case of 6469172, no patents were filled at this stage because the gathered data were considered to provide too little ground for a university to take the financial risk. However, discussions with pharmaceutical companies are planned to see how research could be carried on possibly with other undisclosed molecules.

In summary, the newly-identified compounds presented herein have some promising and innovative application lying ahead, even though the stage of development and characterization is still too early to really predict whether a commercial application could stem from them. 


\section{References}

1. IDF Diabetes Atlas, 6th edn. 2013, International Diabetes Federation: Brussels, Belgium.

2. Kenyon, C.J., The genetics of ageing. Nature, 2010. 464(7288): p. 504-512.

3. Burkewitz, K., Zhang, Y. and Mair, W.B., AMPK at the nexus of energetics and aging. Cell Metab, 2014. 20(1): p. 10-25.

4. Viollet, B., Horman, S., Leclerc, J., Lantier, L., Foretz, M., Billaud, M., Giri, S. and Andreelli, F., $A M P K$ inhibition in health and disease. Crit Rev Biochem Mol Biol, 2010. 45(4): p. 276-295.

5. Rana, S., Blowers, E.C. and Natarajan, A., Small molecule adenosine 5'-monophosphate activated protein kinase (AMPK) modulators and human diseases. J Med Chem, 2015. 58(1): p. 2-29.
6. Hardie, D.G., AMP-activated protein kinase: maintaining energy homeostasis at the cellular and whole-body levels. Annu Rev Nutr, 2014. 34: p. 31-55.

7. Foretz, M., Guigas, B., Bertrand, L., Pollak, M. and Viollet, B., Metformin: from mechanisms of action to therapies. Cell Metab, 2014. 20(6): p. 953-966.

8. Hughes, J.P., Rees, S., Kalindjian, S.B. and Philpott, K.L., Principles of early drug discovery. Br J Pharmacol, 2011. 162(6): p. 1239-1249. 


\section{Curriculum Vitae}

Marie Miglianico was born in Rennes (France) on the $3^{\text {rd }}$ of July 1987. After completion of high school in Saint-Malo (France), she was accepted in the intensive program for preparation to the admission examination to French "Grandes Ecoles" (Lycée Clémenceau, Nantes, France). In 2007, after passing the national selective exam, she was admitted to AgroParisTech, one of France's top engineering schools for life, food and environment sciences (AgroParisTech, Paris, France). During her studies, besides receiving an extensive education in biology, she spent a semester as exchange student in Germany (RWTH, Aachen, Germany), then six months in Burkina Faso on a biofuel project linked with local development (2iE, Ouagadougou, Burkina Faso), and finally another half-year in the USA to study genetic markers of cystic fibrosis patients (Stanford University, CA, USA). After completing her master thesis with the National Museum of Natural History (Paris, France), Marie received a Master of engineering in agronomy as well as a Master degree in "Life science and technology" in 2011. Within the same year, she started as PhD candidate in the group of Dr. Dietbert Neumann at Maastricht University where she also joined the team of Dr. Gerry Nicolaes and received supervision from Prof. dr. Jan Glatz. 


\section{Publications}

Oligschlaeger, Y.*, Miglianico, M.*, Chanda, D., Scholz, R., Thali, R. F., Tuerk, R., Stapleton, D.I., Gooley, P.R., Neumann, D. (2015). The recruitment of AMP-activated protein kinase to glycogen is regulated by autophosphorylation. Journal of Biological Chemistry, 290(18), 11715-11728.

Miglianico, M., Nicolaes, G.A.F., Neumann, D. (In revision). Novel targeting opportunities for AMP-activated protein kinase: isoform specificity and computeraided drug design. Invited in Journal of Medicinal Chemistry.

Oligschlaeger, Y., Miglianico, M., Dahlmans, V., Rubio-Villena, C., Chanda, D., GarciaGimeno, M.A., Liu, Y., Voncken, J.W., Luiken, J.J.F.P., Glatz, J.F.C., Sanz, P., Neumann, D. (Submitted). The interaction between AMPK $\beta 2$ and the PP1-targeting subunit R6 is dynamically regulated by intracellular glycogen content.

Miglianico, M., Oligschlaeger, Y., Chanda, D., Wichapong, K., Coumans, W.A., Jaminon, A., Rodriguez-Calvo, R., Özcete, Ö.D., Schrijver, R., Zhu, X, Bleylevens, I., van den Akker, N.M.S., Molin, D.G.M., Glatz, J.F.C., Nicolaes, G.A.F., Neumann, D. (In preparation). Displacing AMPK from glycogen by pharmacological targeting of its carbohydrate-binding module.

* These authors contributed equally to this study

\section{Poster presentations (selection)}

Nov. 26, 2014 CARIM Annual symposium

Title: Toward selective targeting of muscular AMPK

Oct. 1, 2014 FASEB, AMPK: Biological Action and Therapeutic Perspectives

Title: Toward selective targeting of muscular AMPK

Jun. 25, 2014 Society for Heart and Vascular Metabolism

Title: Toward selective targeting of muscular AMPK

Oct. 25, 2013 Netherlands Society on Biomolecular modeling

Title: Toward selective targeting of muscular AMPK

Oct. 9, 2012 FASEB, AMPK and Beyond

Title: Selective targeting of muscular AMPK by structure based virtual screening

Jun. 25, 2012 Society for Heart and Vascular Metabolism

Title: 'Tour de AMPK': cycling to ameliorate type 2 diabetes?

Apr. 24, 2012 Netherlands Bioinformatics Conference

Title: Selective targeting of muscular AMPK by structure based virtual screening 


\section{Oral presentations}

Nov. 28-29, 2013 Annual Dutch Diabetes Research Meeting

Title: Toward selective targeting of muscular AMPK: in search of a small molecule to change AMPK cellular localization

Sept. 15-17, 2013 European workshop on AMPK

Title: Toward selective targeting of muscular AMPK: in search of a small molecule to change AMPK cellular localization 



\section{Acknowledgments}

To conclude the presentation of the work of these past four years, I would like to acknowledge all the help and support I have received throughout this time. I was lucky enough to be well-surrounded during the past four years, may it be in Maastricht, in Aachen, in France, in Turkey and beyond. Hereby I would like to address my gratitude and my thanks.

My first thought goes to my supervisors. To Jan, many thanks for welcoming me in the department of Molecular Genetics, for your insightful advice, questions and guidance throughout my research and for always doing your best to make our department successful and happy.

Dietbert, not everyone would have entrusted a holder of a Master in Ecology with the design of a novel drug! But you did and for many months (years?) you were maybe more convinced than me that it was a wise choice. I wish that the resulting work reaches your expectation and I want to thank you for this opportunity. I have learned a lot by your side, not only in science but also in seeing you manage our diverse and lively team.

Gerry, you welcomed me into the world of structure biology and drug design. I remember attending a meeting on biomolecular modeling with you within my first weeks of employment... Luckily, it became less of a foreign language later on! Thank you for teaching me and letting me be a part of your team in Biochemistry.

Then I want to express my warm feelings for my dear paranimfs, Barbara and Yvonne. You were both at my side during the fun and the hard times alike. Barbara, you were always there for re-shaping the world around a cup of coffee, and we share some ongelofelijk memories together! Yvonne, we went through the same ups and downs during our four years and I was glad and lucky I could count on my PhD twin sister!

Cheers to the other members of our unstoppable Neumann team! Dip, our big brother, for teaching me to sometimes say "Arf, not necessary" when following protocols. Xiaoqing, for our special moment in the wood of Eifel (!), but mostly for your outgoing and sunny personality. Yilin, for your friendliness and fashion attitude even in a lab coat. Jieyi,you're your kindness and for always laughing at my jokes. Ricardo, our other big brother, for spicing our lab life with theories on who may be a couple. Armand, for taking in my principles on feeding habits without complaints. Laura, for being all at once fun, sweet and open. My students, Özge, Hacer and Magdalena, for being such lovely lab companions and trusting my advice when I was still myself learning. Milou, for your constant energy and good-spirit.

My next thanks go to our MolGen group. Joost, with your cheerful attitude and your inexhaustible knowledge on CD36. Will, what would I have done if you did not teach me to do Western blots?? Yeliz, seninle tanıştığımız için çok mutlu ve şanslıyım. Willem, for 
always coming by at the end of the day just to say "What's up?". Vivian, who volunteered her help when Yvonne and I were swamped with work. Ronit, from whom I learned a lot in our joined Journal Club, and your enthusiastic team: Tim, Sofie, Mike, Patrick and, with a extra word to my sympathetic neighbor, Tom. Guus, Peggy, Nadine, Miranda, Emiel, Marjo, Marion, Petra and Hanneke, I enjoyed sharing Sinterklaas, birthdays and such nice events with you.

Moving on to the members of the Biochemistry department, I think of Roy, who gave me my badges in solvent correction and pipetting compounds for our beloved Biacore; of Jiang, who led me through the labyrinth of FRED, Surflex and so on; of Kanin, our computer expert never too busy to help or discuss; of Karin, a kind and friendly colleague; and of Hans, Farida, Francesca, Daniëlle, Stella, Simone, Betta and many more: thank you for welcoming me in your department.

From elsewhere in the University, to Jo Stevens, to Ivo Bleylevens, to Miriam Kamps, to Nynke van den Akker, to Daniel Molin, to Mick Gagliardi, to Mat Rousch, to Johan Heemskerk, to Marijke Kuijpers, I send my thanks for your advice, help and teaching.

Dear Professor Tilman Hackeng, Doctor Matthijs Blankensteijn, Professor HansPeter Brunner-LaRocca, Professor Uwe Schlattner and Professor Bert Smeets, I am grateful to you for joining the assessing committee, revising my thesis and granting it approval.

On a more personal note now, I thank all my friends who will recognize themselves in these lines: those already mentioned above, those in the Netherlands, in France, in Germany, in Turkey and beyond, those I know since high school and those who are more recent additions. These four years were full of memorable weekends, evenings and day-trips thanks to you all I wish you will stay by my side longer still.

Ma pensée va ensuite vers ma famille, tellement pleine de personnes qui m'inspirent, me soutiennent, m'encouragent. Même loin des yeux, nous restons proches et cela compte beaucoup pour moi. Merci pour votre soutien indéfectible au cours de ces nombreuses (pas seulement quatre) années ! Kanpolat ailesine, her zaman bana çok nazik oldukları için çok teşekkür ederim.

Finally, to Emre Kanpolat, mon compagnon de vie, de voyage et bien plus. Emrecim, thank you for making it so easy for me, for always knowing when to let me work and when to make me take a break. Beyond your priceless help and support, you made these years colorful with your many plans, ideas and energy. I am now looking forward to our new adventure! İyiki varsın! 